$$
\text { UNITED STATES }
$$

DEPARTMENT OF THE INTERIOR

GEOLOGICAL SURVEY

\title{
HYDROGEOLOGIC DATA FOR ROCKY CREEK LANDFILL AND ADJACENT AREA, NORTHWEST HILLSBOROUGH COUNTY, FLORIDA, 1969-73
}

OPEN-FILE REPORT $80-1291$

Prepared in cooperation with

HILLSBOROUGH COUNTY and the

CITY of TAMPA, FLORIDA 
For those readers who prefer to use metric (SI) units rather than inchpound units, conversion factors for terms used in this report are 1 isted below:

Multiply inch-pound unit

inch (in.)

foot (ft)

mile (mi)
By

25.4

0.3048

1.609
To obtain metric (SI) unit millimeter (mm) meter (m)

kilometer $(\mathrm{km})$

National Geodetic Vertical Datum of 1929 (NGVD of 1929).--A geodetic datum derived from a general adjustment of the first-order level nets of both the United States and Canada, formerly called "mean sea level." 


\section{UNITED STATES \\ DEPARTMENT OF THE INTERIOR \\ GEOLOGICAL SURVEY}

HYDROGEOLOGIC DATA FOR ROCKY CREEK LANDFILL AND ADJACENT

AREA, NORTHWEST HILLSBOROUGH COUNTY, FLORIDA, 1969-73

By A. D. Duerr and J. W. Stewart

Open-File Report 80-1291

Prepared in cooperation with

HILLSBOROUGH COUNTY and the

CITY of TAMPA, FLORIDA

Tallahassee, Florida 
UNITED STATES DEPARTMENT OF THE INTERIOR

CECIL D. ANDRUS, Secretary

GEOLOGICAL SURVEY

H. William Menard, Director

For additional information write to:

U.S. Geological Survey

325 John Knox Road, Suite F-240

Tallahassee, Florida 32303 
Abstract - Introduction -

Previous investigations -_-_-_-_-_-_-_-_ 5

Hydrogeologic data -

References -_-_-_-_-_-_-_-_-_ 5

\section{ILLUSTRATIONS}

Figure 1. Map showing location of Rocky Creek landfill in northwest Hillsborough County --_-_- 2

2. Map showing location of ground-water sites in northwest Hillsborough County - 3

3. Map showing location of landfill trenches, ground-water sites, and surface-water sites at the Rocky Creek landfill _-_... 4

TABLES

Table 1. Records of wells in northwest Hillsborough County ______ 6

2. Water-level measurements in wells in northwest Hillsborough County - 12

3. Lithologic logs of wells in northwest Hillsborough County ---- 29

4. Surface-water sites at the Rocky Creek landfill 39

5. Water-quality data for surface-water sites at the Rocky Creek landfill - 40

6a. Water-quality data for ground-water sites at the Rocky Creek landfill - 44

$6 \mathrm{~b}$. Water-quality data for ground-water sites at the Rocky Creek landfill - 55

7. Nitrogen and phosphorus data for ground-water sites at the Rocky Creek landfill - 65

8. Trace-metals data for ground-water sites at the Rocky Creek landfill - 67

9. BOD, carbon, and coliform bacteria data for ground-water sites at the Rocky Creek landfill 71 



\section{HYDROGEOLOGIC DATA FOR ROCKY CREEK LANDFILL AND ADJACENT AREA,}

NORTHWEST HILLSBOROUGH COUNTY, FLORIDA, 1969-73

By A. D. Duerr and J. W. Stewart

ABSTRACT

We11 location and construction data are summarized for 222 we11s in the Rocky Creek landfill and adjacent area in northwest Hillsborough County. Most of the data are for 92 wells within the landfill or immediate vicinity. Waterquality data are presented for 78 wells and nine surface-water sites. Waterlevel data for 133 wells penetrating the surficial and Floridan aquifers and lithologic logs for 35 wells are also presented.

\section{INTRODUCTION}

In 1969, Hillsborough County and the city of Tampa entered into a cooperative program with the U.S. Geological Survey to monitor water quality and water levels at the county's Rocky Creek landfill in northwest Hillsborough County (fig. 1). Data included in this report were collected from January 1969 through December 1973. Selected wells within 5 miles of the landfill were inventoried and used to monitor ground-water levels in the area (fig. 2). Locations of 42 public-supply wells are also shown in figure 2. Ninety-two test wells open to the surficial aquifer or to the Floridan aquifer were constructed at the landfill site and adjacent area to establish a monitoring network (fig. 3). An additional 22 test wells were constructed outside the landfill site. During the period of study, 31 trenches averaging 400 feet 1 ong, 125 feet wide, and 10 feet deep were excavated and filled with solid waste at the landfill site. In addition, two mounds of solid waste 20 feet high were constructed over filled trenches and covered with dirt (see fig 3 ).

The purpose of this report is to present hydrologic, geologic, and waterquality data collected at the Rocky Creek landfill and adjacent area from January 1969 through December 1973. Hydrologic and/or water-quality data are included for 222 wells and nine surface-water sites within a $100 \mathrm{mi}^{2}$ area in northwest Hillsborough County. Most water-quality data were obtained for test wells within the Rocky Creek landfill site. The report includes lithologic logs of 35 observation wells in the area. 


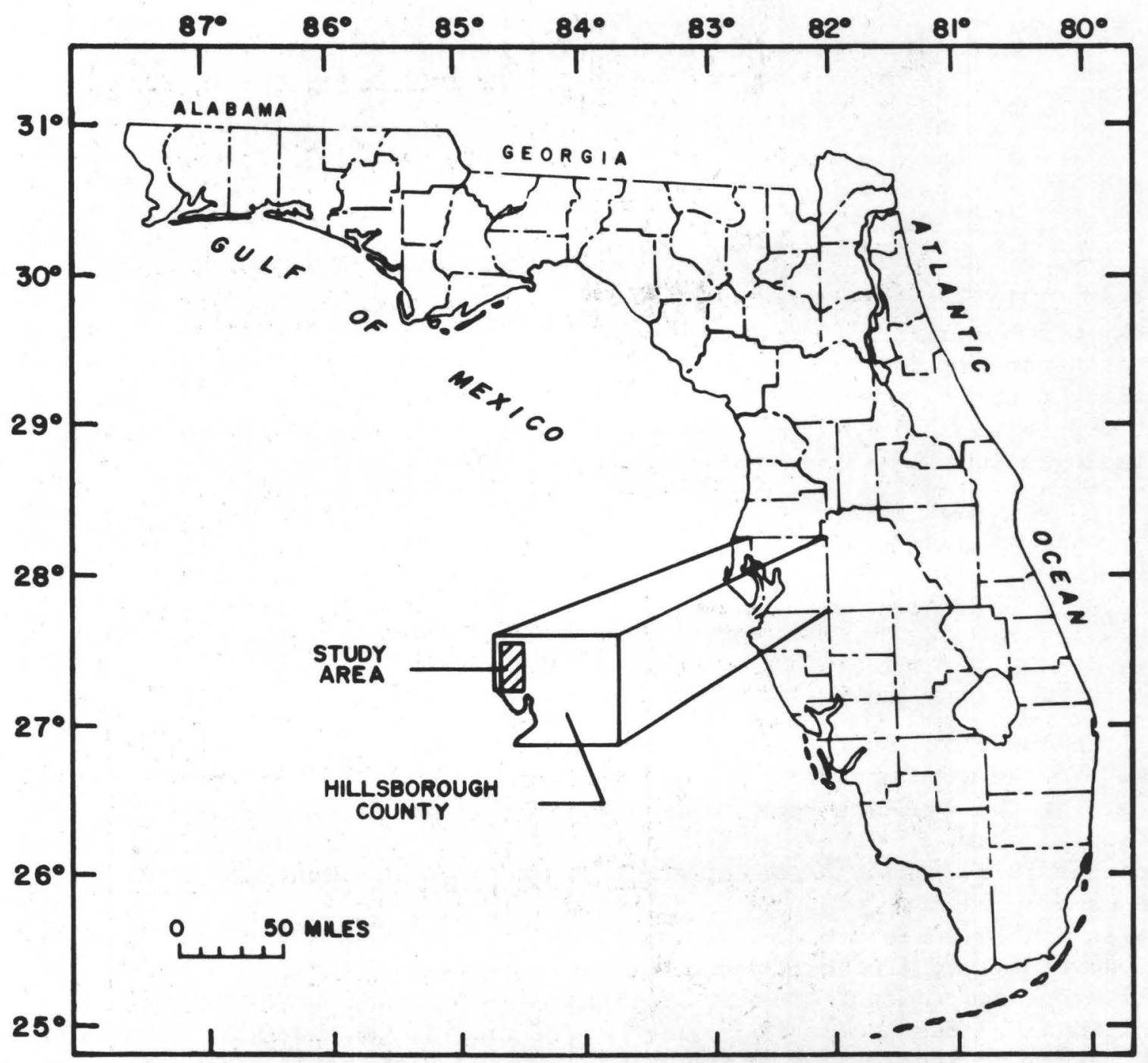

Figure 1.--Location of Rocky Creek landfill in northwest Hillsborough County. 


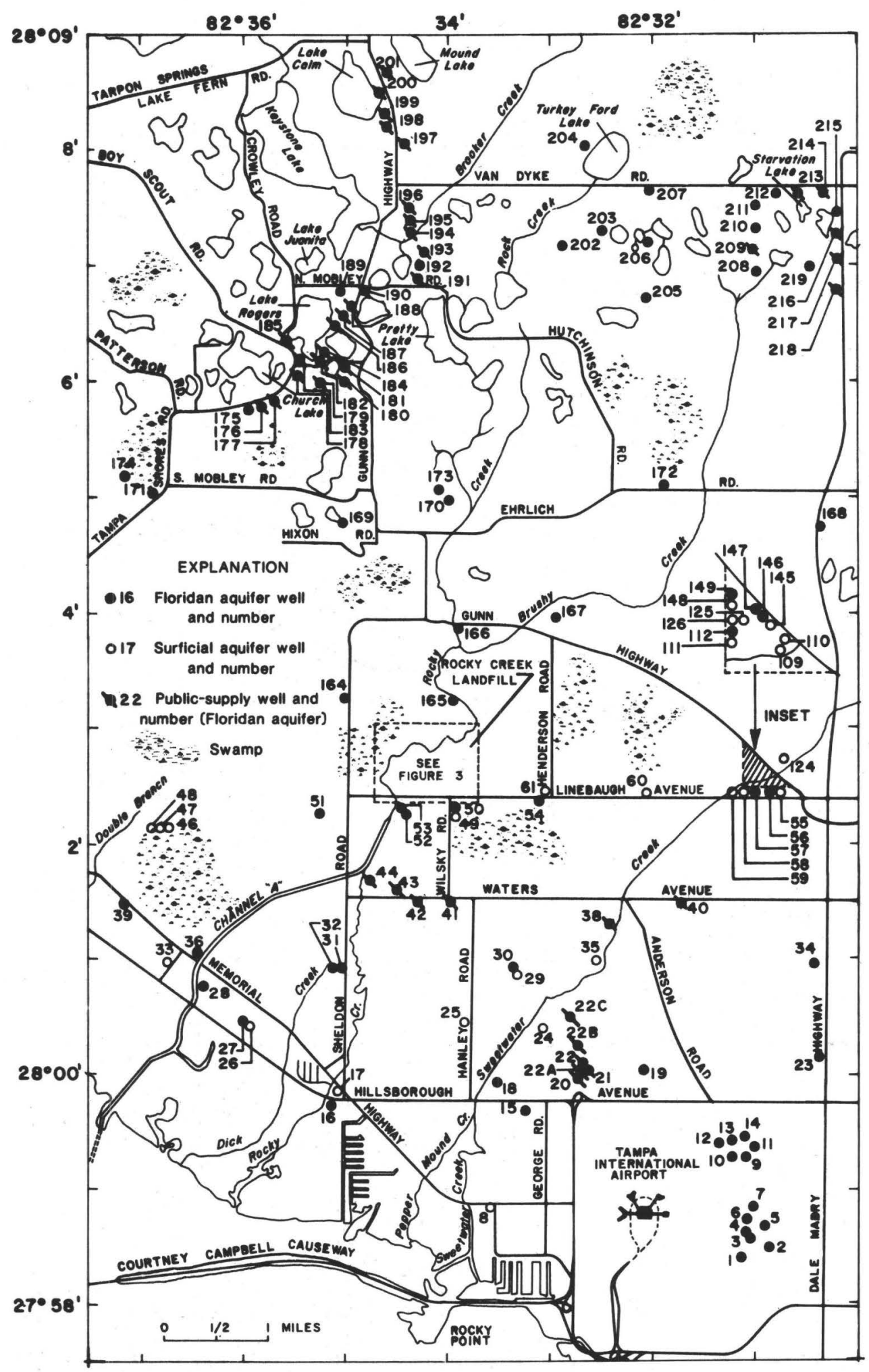

Figure 2.--Location of ground-water sites in northwest Hillsborough County. 
$22^{\circ} 30^{\circ} 30^{\circ}$

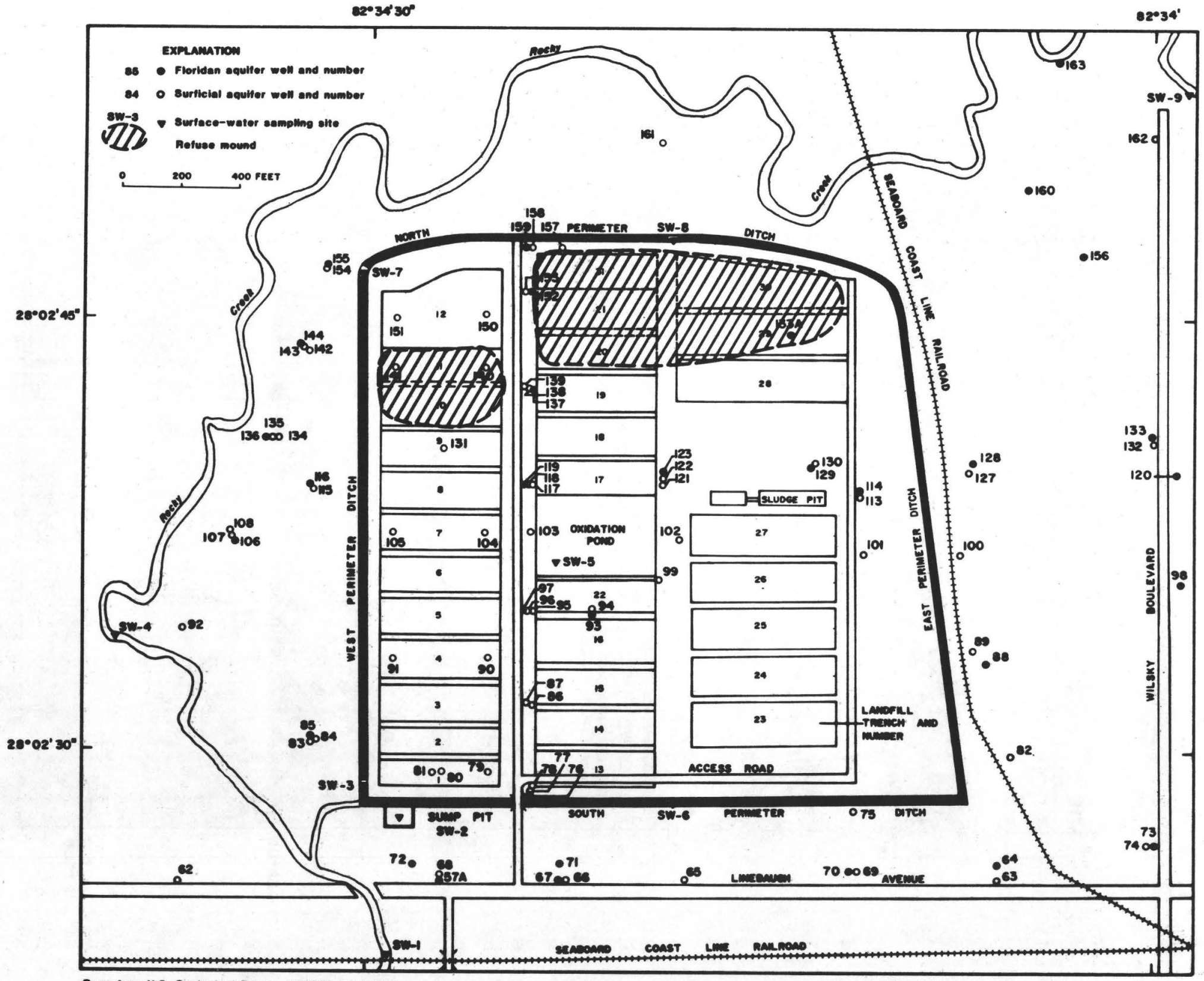

Figure 3.--Location of landfill trenches, ground-water sites, and surface-water sites at the Rocky Creek landfill. 
Information on several wells in the area was included in a report on Hillsborough County by Menke and others (1964). Stewart and Hanan (1970) prepared a map of the north half of Hillsborough County showing areas of artesian flow, areas of sinkholes, the potentiometric surface of the Floridan aquifer, a generalized water-table map, thickness of surficial sediments, public-water supplies, and landfill sites. Stewart and Duerr (1973) showed how hydrology and geology are applied in evaluating landfill sites in west-central Florida. Many of the photographs in that report were taken at the Rocky Creek landfill. Fernandez and Hallbourg (1979) presented hydrogeologic data for wells at the Rocky Creek landfill for the period 1974-77.

\section{HYDROGEOLOGIC DATA}

Table 1 presents location, aquifer, depth, casing depth and size, screened interval, and land-surface altitude for 222 wells in northwest Hillsborough County. The wells range in depth from 5 to 1,250 feet. Eighty-seven wells were constructed in the surficial aquifer and 135 tap the Floridan aquifer. Well locations are shown in figures 2 and 3.

Table 2 lists water levels collected during the period 1969-73 for 133 wells in the surficial and Floridan aquifers. Water levels in the Floridan aquifer within the landfill area (fig. 3) ranged from 13.3 to 24.1 feet above NGVD of 1929 (National Geodetic Vertical Datum of 1929). Water levels in the surficial aquifer ranged from 4.0 to 25.2 feet above the datum. Water levels in both aquifers were higher in the eastern part of the landfill than in the western part.

Table 3 presents lithologic logs for 35 wells, most of which are within or near the landfill. These logs show that unconsolidated deposits of sand and clay are about 30 to 75 feet thick overlying the Floridan aquifer. Table 4 lists the site number and site name for nine surface-water sites. The locations are shown in figure 3. Water-quality data for the nine sites are listed in table 5. Table 6 lists water-quality data for 78 ground-water sites. Table 7 lists nitrogen and phosphorus, table 8 lists trace-metals data, and table 9 lists BOD, carbon, and coliform bacteria for the ground-water sites.

\section{REFERENCES}

Fernandez, Mario, Jr., and Hallbourg, R. B., 1979, Water-quality data for landfills, Hillsborough County, Florida, January 1974-October 1977: U.S. Geological Survey Open-File Report 78-820, 112 p.

Menke, C. G., Meredith, E. W., and Wetterha11, W. S., 1964, Water resources records of Hillsborough County, Florida: Florida Geological Survey Information Circular 44, 95 p.

Stewart, J. W., and Duerr, A. D., 1973, Hydrologic and geologic considerations for solid-waste disposal in west-central Florida: U.S. Geological Survey Water-Resources Investigations 50-73, 52 p.

Stewart, J. W., and Hanan, R. V., 1970, Hydrologic factors affecting the utilization of land for sanitary landfills in northern Hillsborough County, Florida: Florida Bureau of Geology Map Series 39. 
Table 1.--Records of wells in northwest Hillsborough County

\begin{tabular}{|c|c|c|c|c|c|c|c|c|c|}
\hline \multirow{2}{*}{$\begin{array}{l}\text { We11 } \\
\text { num } \\
\text { ber }\end{array}$} & \multirow{2}{*}{$\begin{array}{c}\text { Identification } \\
\text { number }\end{array}$} & \multirow{2}{*}{$\begin{array}{l}\text { Aquij } \\
\text { fer }\end{array}$} & \multirow{2}{*}{$\begin{array}{c}\text { Depth } \\
\text { of } \\
\text { we11 }- \\
(f t)\end{array}$} & \multicolumn{2}{|c|}{ Casing } & \multicolumn{2}{|c|}{$\begin{array}{l}\text { Screen } \\
(f t)\end{array}$} & \multirow{2}{*}{$\begin{array}{l}\text { Land } \\
\text { surface } \\
\text { alti } \overline{4} / \\
\text { tude } \\
\text { (ft) }\end{array}$} & \multirow{2}{*}{ Remarks } \\
\hline & & & & $\begin{array}{l}\text { Depth }{ }^{3 /} \\
(\mathrm{ft})\end{array}$ & $\begin{array}{l}\text { Diam- } \\
\text { eter } \\
\text { (in.) }\end{array}$ & From & To & & \\
\hline 1 & 2758230823121.01 & F & 312 & 40 & 6 & - & - & 20.6 & City of Tampa 1 \\
\hline 2 & 2758290823054.01 & $\mathbf{F}$ & 512 & 58 & 10 & - & - & 31 & City of Tampa 14 \\
\hline 3 & 2758340823104.01 & $\mathbf{F}$ & 230 & -- & 10 & -- & - & 28 & City of Tampa 10 \\
\hline 4 & 2758370823108.01 & $\mathbf{F}$ & 513 & 135 & 10 & -- & -- & 27 & City of Tampa 6 \\
\hline 5 & 2758400823056.01 & $\mathbf{F}$ & 436 & 141 & 10 & -- & -- & 29.5 & City of Tampa 5 \\
\hline 6 & 2758430823106.01 & $\mathbf{F}$ & 502 & 99 & 10 & -- & - & 27.9 & City of Tampa 12 \\
\hline 7 & 2758500823101.01 & $\mathbf{F}$ & 508 & 93 & 10 & -- & -- & 28.1 & City of Tampa 5 \\
\hline 8 & 2758520823333.01 & $\mathbf{s}$ & 16.0 & 14.0 & $11 / 4$ & 14.0 & 16.0 & 7 & Memorial Hwy 14 \\
\hline 9 & 2759160823107.01 & $\mathbf{F}$ & 501 & 84 & 12 & -- & - & 32.8 & City of Tampa 16 \\
\hline 10 & 2759160823115.01 & F & 511 & 113 & 10 & -- & -- & 29.7 & City of Tampa 7 \\
\hline 11 & 2759220823102.01 & $\mathbf{F}$ & 500 & 74 & 12 & -- & - & 28.3 & City of Tampa 15 \\
\hline 12 & 2759220823123.01 & $\mathbf{F}$ & 337 & 94 & 10 & -- & -- & 28.3 & City of Tampa 13 \\
\hline 13 & 2759240823116.01 & F & 208 & 87 & 10 & - & -- & 32 & City of Tampa 8 \\
\hline 14 & 2759270823108.01 & $\mathbf{F}$ & 478 & 121 & 10 & -- & - & 28.6 & City of Tampa 9 \\
\hline 15 & 2759420823317.01 & $\mathbf{F}$ & 200 & 50 & 4 & -- & - & 10 & \\
\hline 16 & 2759420823508.01 & F & 107 & 30 & 2 & -- & - & 5 & \\
\hline 17 & 2759450823451.01 & $\mathbf{s}$ & 25.0 & 23.5 & $11 / 4$ & 23.5 & 25.0 & 5.2 & \\
\hline 18 & 2759560823334.01 & $\mathbf{F}$ & -- & -- & 2 & -- & - & 9 & \\
\hline 19 & 2759590823208.01 & $\mathbf{F}$ & 40.0 & -- & 2 & -- & -- & 24 & \\
\hline 20 & 2800010823241.01 & $\mathbf{F}$ & 303 & 112 & 8 & -- & -- & 20 & Suburban Util. 2 \\
\hline 21 & 2800030823241.01 & $\mathbf{F}$ & 185 & 97 & 6 & -- & - & 20 & Suburban Util. 3 \\
\hline 22 & 2800050823241.01 & $\mathbf{F}$ & 202 & 105 & 8 & - & -- & 20 & Suburban Util. 1 \\
\hline $22 a$ & 2800030823242.01 & $\mathbf{F}$ & 200 & 92 & 10 & -- & - & 20 & Suburban Util. 4 \\
\hline $22 b$ & 2800110823243.01 & $\mathbf{F}$ & 405 & 95 & 12 & - & - & 20 & Suburban Util. 5 \\
\hline $22 \mathrm{c}$ & 2800230823245.01 & $\mathbf{F}$ & 400 & 100 & 12 & - & - & 20 & Suburban Util. 6 \\
\hline 23 & 2800070823028.01 & $\mathbf{F}$ & 150 & -- & -- & -- & -- & 30 & \\
\hline 24 & 2800260823259.01 & $\mathbf{s}$ & 9.0 & 7.0 & $11 / 4$ & 7.0 & 9.0 & 18 & \\
\hline 25 & 2800280823347.01 & $\mathbf{s}$ & 8.2 & 6.2 & $11 / 4$ & 6.2 & 8.2 & 12 & \\
\hline 26 & 2800290823558.02 & $\mathbf{s}$ & 18.0 & 16.5 & 2 & 16.5 & 18.0 & 8 & Pistol Club 9A \\
\hline 27 & 2800290823558.01 & F & 50.0 & 48.5 & 2 & 48.5 & 50.0 & 8 & Pistol Club 9 \\
\hline 28 & 280047087.3626 .01 & $\mathbf{F}$ & 49.5 & 48.0 & 2 & 48.0 & 49.5 & 8 & Pistol Club 11 \\
\hline 29 & 2800520823322.02 & s & 19.8 & 15.8 & 2 & 15.8 & 19.8 & 15 & \\
\hline 30 & 2800520823322.01 & $\mathbf{F}$ & 39.2 & 35.2 & 2 & 35.2 & 39.2 & 15 & \\
\hline 31 & 2800530823502.02 & $\mathbf{F}$ & 330.0 & 315.0 & 6 & -- & - & 8.9 & \\
\hline 32 & 2800530823502.01 & F & 88.0 & 39.0 & 6 & - & - & 8.9 & \\
\hline 33 & 2800540823643.01 & $\mathbf{s}$ & 22.0 & 20.5 & 2 & 20.5 & 22.0 & 6 & Pistol Club 7 \\
\hline 34 & 2800570823026.01 & $\mathbf{F}$ & 150 & - & 3 & - & -- & 35 & \\
\hline 35 & 2800580823233.01 & $\mathbf{S}$ & 9.3 & 7.3 & $11 / 4$ & 7.3 & 9.3 & 22.2 & \\
\hline 36 & 2800580823624.01 & $\mathbf{F}$ & 90.0 & 88.5 & 2 & 88.5 & 90.0 & 10.1 & Pistol Club 2 \\
\hline 38 & 2801170823227.01 & $\mathbf{F}$ & 260 & 102 & 12 & - & - & 23.3 & City of Tampa \\
\hline
\end{tabular}

Footnotes are at end of table. 
Table 1.--Records of wells in northwest Hillsborough County--Continued

\begin{tabular}{|c|c|c|c|c|c|c|c|c|c|}
\hline \multirow{2}{*}{$\begin{array}{l}\text { We11 } \\
\text { num } \\
\text { ber }^{\prime}\end{array}$} & \multirow{2}{*}{$\begin{array}{c}\text { Identification } \\
\text { number }\end{array}$} & \multirow{2}{*}{$\begin{array}{l}\text { Aquij }_{2} \\
\text { fer }\end{array}$} & \multirow{2}{*}{$\begin{array}{c}\text { Depth } \\
\text { of } \\
\text { we11- } \\
(f t)\end{array}$} & \multicolumn{2}{|c|}{ Casing } & \multicolumn{2}{|c|}{$\begin{array}{l}\text { Screen } \\
(\mathrm{ft})\end{array}$} & \multirow{2}{*}{$\begin{array}{l}\text { Land } \\
\text { surface } \\
\text { alt } i_{\overline{4}} / \\
\text { tude } \\
\text { (ft) }\end{array}$} & \multirow{2}{*}{ Remarks } \\
\hline & & & & $\begin{array}{l}\text { Depth }{ }^{3 /} \\
(\mathrm{ft})\end{array}$ & $\begin{array}{l}\text { Diam- } \\
\text { eter } \\
\text { (in.) }\end{array}$ & From & To & & \\
\hline 39 & 2801280823708.01 & $\mathbf{F}$ & -- & - & 2 & -- & -- & 5 & Keys Ranch \\
\hline 40 & 2801290823147.01 & F & 405.0 & 90.0 & 12 & -- & -- & 28.6 & City of Tampa \\
\hline 41 & 2801310823401.01 & $\mathbf{F}$ & 180.0 & 82.0 & 8 & -- & -- & 17 & River Oaks 3 \\
\hline 42 & 2801310823420.01 & $\mathbf{F}$ & 174.0 & 65.0 & 8 & -- & -- & 15 & River Oaks 2 \\
\hline 43 & 2801330823428.01 & F & 94.0 & 60.0 & 8 & -- & -- & 16 & River Oaks 1 \\
\hline 44 & 2801410823444.01 & $\mathbf{F}$ & 84.0 & -- & - & - & - & 10 & River Oaks 4 \\
\hline 46 & 2802070823647.01 & s & 21.3 & -- & $11 / 4$ & -- & -- & 10 & \\
\hline 47 & 2802070823648.02 & s & 15.6 & -- & $11 / 4$ & - & -- & 10 & \\
\hline 48 & 2802070823648.01 & S & 21.6 & - & $11 / 4$ & -- & -- & 10 & \\
\hline 49 & 2802160823358.02 & s & 18.0 & 16.0 & 2 & 16.0 & 18.0 & 24.0 & \\
\hline 50 & 2802160823358.01 & $\mathbf{F}$ & 46.5 & 46.0 & 2 & -- & -- & 24.2 & \\
\hline 51 & 2802190823516.01 & $\mathbf{F}$ & -- & - & 2 & -- & -- & 15 & \\
\hline 52 & 2802190823428.01 & $\mathbf{F}$ & 200 & -- & 2 & - & -- & 15 & Zambito \\
\hline 53 & 2802210823429.01 & $\mathbf{F}$ & 58 & -- & 2 & -- & -- & 15 & Zambito \\
\hline 54 & 2802220823306.01 & $\mathbf{F}$ & -- & -- & 2 & -- & -- & 27 & \\
\hline 55 & 2802230823054.02 & s & 10.0 & 6.0 & 2 & 6.0 & 10.0 & 32.4 & \\
\hline 56 & 2802230823054.01 & F & 42.8 & 38.8 & 2 & 38.8 & 42.8 & 32.3 & \\
\hline 57 & 2802240823108.01 & $\mathbf{F}$ & 37.0 & 33.0 & 2 & 33.0 & 37.0 & 36.3 & \\
\hline $57 a$ & 2802260823429.01 & $\mathbf{F}$ & 43 & -- & - & - & - & 15.1 & Test hole only \\
\hline 58 & 2802240823108.02 & $\mathrm{~s}$ & 15.0 & 11.0 & 2 & 11.0 & 15.0 & 35.6 & \\
\hline 59 & 2802240823116.01 & s & 8.8 & 6.8 & $11 / 4$ & 6.8 & 8.8 & 33.4 & \\
\hline 60 & 2802250823202.01 & $\mathbf{s}$ & 9.2 & 7.2 & $11 / 4$ & 7.2 & 9.2 & 34.3 & \\
\hline 61 & 2802250823301.01 & $\mathrm{~s}$ & 8.5 & 6.5 & $11 / 4$ & 6.5 & 8.5 & 28.6 & \\
\hline 62 & 2802250823438.01 & s & 9.0 & 7.0 & $11 / 4$ & 7.0 & 9.0 & 18 & \\
\hline 63 & 2802260823407.01 & s & 9.0 & 7.0 & $11 / 4$ & 7.0 & 9.0 & 22.5 & \\
\hline 64 & 2802260823407.02 & $\mathbf{F}$ & 90.0 & 85.0 & 2 & 85.0 & 90.0 & 24.7 & \\
\hline 65 & 2802260823421.01 & $\mathbf{s}$ & 12.0 & 10.0 & $11 / 4$ & 10.0 & 12.0 & 19.6 & \\
\hline 66 & 2802260823425.02 & $\mathbf{s}$ & 9.6 & 7.6 & 2 & 7.6 & 9.6 & 16.8 & \\
\hline 67 & 2802260823425.01 & $\mathbf{F}$ & 40.3 & 40.0 & 2 & -- & -- & 17.1 & \\
\hline 68 & 2802260823429.02 & $\mathrm{~s}$ & 10.0 & 8.0 & 2 & 8.0 & 10.0 & 15.1 & \\
\hline 69 & 2802260823412.02 & S & 15.7 & 10.7 & 2 & 10.7 & 15.7 & 22.3 & \\
\hline 70 & 2802260823412.01 & $\mathbf{F}$ & 65.5 & 60.5 & 2 & 60.5 & 65.5 & 22.5 & \\
\hline 71 & 2802260823425.04 & $\mathbf{F}$ & 127 & -- & 3 & -- & -- & 17 & \\
\hline 72 & 2802270823430.01 & $\mathbf{F}$ & 47.0 & -- & 2 & -- & - & 15.1 & \\
\hline 73 & 2802280823359.01 & $\mathbf{F}$ & 67.0 & 67 & 2 & - & - & 24.4 & \\
\hline 74 & 2802280823359.02 & s & 18.0 & 16.0 & 2 & 16.0 & 18.0 & 24.3 & \\
\hline 75 & 2802280823412.01 & s & 14.9 & 9.9 & 2 & 9.9 & 14.9 & 22.0 & \\
\hline 76 & 2802280823426.01 & $\mathbf{F}$ & 80.0 & 80.0 & 2 & -- & - & 19.4 & \\
\hline 77 & 2802280823426.02 & $\mathrm{~s}$ & 9.3 & 7.3 & 2 & 7.3 & 9.3 & 19.3 & \\
\hline 78 & 2802280823426.03 & $\mathbf{s}$ & 24.4 & 22.4 & 2 & 22.4 & 24.4 & 18.8 & \\
\hline
\end{tabular}


Table 1.--Records of wells in northwest Hillsborough County--Continued

\begin{tabular}{|c|c|c|c|c|c|c|c|c|c|}
\hline \multirow{2}{*}{$\begin{array}{l}\text { We11 } \\
\text { num }_{\overline{1}} / \\
\text { ber- }^{\prime}\end{array}$} & \multirow{2}{*}{$\begin{array}{c}\text { Identification } \\
\text { number }\end{array}$} & \multirow{2}{*}{$\begin{array}{l}\text { Aquit } \\
\text { fer }\end{array}$} & \multirow{2}{*}{$\begin{array}{c}\text { Depth } \\
\text { of } \\
\text { we11 } 3 / \\
(f t)\end{array}$} & \multicolumn{2}{|c|}{ Casing } & \multicolumn{2}{|c|}{$\begin{array}{l}\text { Screen } \\
(\mathrm{ft})\end{array}$} & \multirow{2}{*}{$\begin{array}{l}\text { Land } \\
\text { surface } \\
\text { alti } \bar{T}_{\overline{4}} / \\
\text { tude }^{-} \\
\text {(ft) }\end{array}$} & \multirow{2}{*}{ Remarks } \\
\hline & & & & $\begin{array}{l}\text { Depth-3/ } \\
(\mathrm{ft})\end{array}$ & $\begin{array}{l}\text { Diam- } \\
\text { eter } \\
\text { (in.) }\end{array}$ & From & To & & \\
\hline 79 & 2802280823427.01 & $\mathbf{S}$ & 14.0 & 11.5 & 2 & 11.5 & 14.0 & 21.8 & \\
\hline 80 & 2802280823428.01 & $\mathrm{~s}$ & 13.0 & 9.0 & 4 & 9.0 & 13.0 & 22.1 & \\
\hline 81 & 2802280823428.02 & $\mathbf{s}$ & 5.0 & 5.0 & 4 & -- & -- & 22.1 & \\
\hline 82 & 2802300823405.01 & $\mathrm{~s}$ & 19.5 & 15.5 & 2 & 15.5 & 19.5 & 25.0 & \\
\hline 83 & 2802310823436.03 & s & 13.9 & 11.9 & 2 & 11.9 & 13.9 & 16.9 & \\
\hline 84 & 2802310823436.02 & S & 24.0 & 22.0 & 2 & 22.0 & 24.0 & 17.2 & \\
\hline 85 & 2802310823436.01 & F & 50.5 & 50.0 & 2 & -- & - & 16.8 & \\
\hline 86 & 2802310823426.02 & $\mathrm{~S}$ & 23.0 & 21.0 & 2 & 21.0 & 23.0 & 21.0 & \\
\hline 87 & 2802310823426.01 & $\mathrm{~s}$ & 8.3 & 6.3 & 2 & 6.3 & 8.3 & 20.7 & \\
\hline 88 & 2802330823407.02 & $\mathrm{~F}$ & 65.4 & 60.4 & 2 & 60.4 & 65.4 & 24.9 & \\
\hline 89 & 2802330823407.01 & $\mathrm{~S}$ & 14.6 & 12.6 & 2 & 12.6 & 14.6 & 24.8 & \\
\hline 90 & 2802330823427.01 & $\mathrm{~s}$ & 14.0 & 11.5 & 2 & 11.5 & 14.0 & 24.0 & \\
\hline 91 & 2802330823429.01 & $\mathrm{~s}$ & 14.5 & 12.0 & 2 & 12.0 & 14.5 & 24.1 & \\
\hline 92 & 2802360823439.02 & $\mathrm{~s}$ & 11.5 & 9.5 & 2 & 9.5 & 11.5 & 9.7 & \\
\hline 93 & 2802330823423.01 & F & 76.3 & 76.3 & $11 / 4$ & -- & -- & 19.3 & Destroyed 1971 \\
\hline 94 & 2802330823423.02 & s & 17.0 & 15.0 & 2 & 15.0 & 17.0 & 19.5 & Destroyed 1971 \\
\hline 95 & 2802330823426.03 & $\mathrm{~s}$ & 9.3 & 7.3 & 2 & 7.3 & 9.3 & 21.9 & \\
\hline 96 & 2802330823426.02 & $\mathrm{~s}$ & 23.4 & 21.4 & 2 & 21.4 & 23.4 & 21.9 & \\
\hline 97 & 2802330823426.01 & F & 52.7 & 50.7 & 2 & 50.7 & 52.7 & 22.2 & \\
\hline 98 & 2802350823358.01 & F & 65 & -- & -- & -- & -- & 25 & \\
\hline 99 & 2802350823417.01 & S & 22.0 & 20.0 & 4 & 20.0 & 22.0 & 22.3 & \\
\hline 100 & 2802360823407.01 & $\mathrm{~s}$ & 19.3 & 14.3 & 2 & 14.3 & 19.3 & 25.4 & \\
\hline 101 & 2802360823409.01 & $\mathrm{~s}$ & 22.0 & 17.0 & 2 & 17.0 & 22.0 & 22.4 & \\
\hline 102 & 2802350823417.02 & $\mathrm{~s}$ & 22.0 & 18.0 & 2 & 18.0 & 22.0 & 24.1 & Destroyed 1973 \\
\hline 103 & 2802360823426.01 & S & 21.0 & 16.5 & 4 & 16.5 & 21.0 & 23.9 & \\
\hline 104 & 2802370823427.01 & $\mathrm{~s}$ & 14.0 & 11.5 & 2 & 11.5 & 14.0 & 24.6 & \\
\hline 105 & 2802370823429.01 & S & 14.5 & 12.0 & 2 & 12.0 & 14.5 & 21.9 & \\
\hline 106 & 2802370823438.01 & $\mathbf{F}$ & 49.4 & 47.4 & 2 & 47.4 & 49.4 & 20.9 & \\
\hline 107 & 2802370823438.02 & $\mathrm{~s}$ & 23.5 & 21.5 & 2 & 21.5 & 23.5 & 21.0 & \\
\hline 108 & 2802370823438.03 & $\mathrm{~s}$ & 14.5 & 12.5 & 2 & 12.5 & 14.5 & 20.7 & \\
\hline 109 & 2802380823051.01 & $\mathrm{~s}$ & 29.0 & 25.0 & 2 & 25.0 & 29.0 & 37.4 & \\
\hline 110 & 2802380823051.02 & $\mathrm{~s}$ & 13.0 & 9.0 & 2 & 9.0 & 13.0 & 37.4 & \\
\hline 111 & 2802380823102.02 & $\mathrm{~s}$ & 10.8 & 6.8 & 2 & 6.8 & 10.8 & 37.3 & \\
\hline 112 & 2802380823102.01 & $\mathbf{F}$ & 28.5 & 24.5 & 2 & 24.5 & 28.5 & 37.3 & \\
\hline 113 & 2802380823409.02 & $\mathrm{~s}$ & 22.0 & 17.0 & 2 & 17.0 & 22.0 & 24.0 & \\
\hline 114 & 2802380823409.01 & F & 46.2 & 41.2 & 2 & 41.2 & 46.2 & 23.9 & \\
\hline 115 & 2802380823432.02 & $\mathrm{~s}$ & 13.5 & 11.5 & 2 & 11.5 & 13.5 & 19.4 & \\
\hline 116 & 2802380823432.01 & F & 43.5 & 41.5 & 2 & 41.5 & 43.5 & 19.8 & \\
\hline 117 & 2802380823426.03 & $\mathrm{~s}$ & 9.1 & 7.1 & 2 & 7.1 & 9.1 & 22.6 & \\
\hline 118 & 2802380823426.02 & s & 24.0 & 22.0 & 2 & 22.0 & 24.0 & 22.6 & \\
\hline
\end{tabular}


Table 1.--Records of wells in northwest Hillsborough County--Continued

\begin{tabular}{|c|c|c|c|c|c|c|c|c|c|}
\hline \multirow{2}{*}{$\begin{array}{l}\text { We11 } \\
\text { num }_{\overline{1}} / \\
\text { ber- }\end{array}$} & \multirow{2}{*}{$\begin{array}{c}\text { Identification } \\
\text { number }\end{array}$} & \multirow{2}{*}{$\begin{array}{l}\text { Aquij } \\
\text { fer }\end{array}$} & \multirow{2}{*}{$\begin{array}{l}\text { Depth } \\
\text { of } \\
\text { we11 } 3 / \\
(f t)\end{array}$} & \multicolumn{2}{|c|}{ Casing } & \multicolumn{2}{|c|}{$\begin{array}{l}\text { Screen } \\
\text { (ft) }\end{array}$} & \multirow{2}{*}{$\begin{array}{l}\text { Land } \\
\text { surface } \\
\text { alti } \overline{4} / \\
\text { tude- } \\
\text { (ft) }\end{array}$} & \multirow{2}{*}{ Remarks } \\
\hline & & & & $\begin{array}{l}\text { Depth }{ }^{3 /} \\
\text { (ft) }\end{array}$ & $\begin{array}{l}\text { Diam- } \\
\text { eter } \\
\text { (in.) }\end{array}$ & From & To & & \\
\hline 119 & 2802380823426.01 & $\mathrm{~F}$ & 41.8 & 39.8 & 2 & 39.8 & 41.8 & 22.0 & \\
\hline 120 & 2802380823357.01 & $\mathrm{~F}$ & 150 & -- & -- & -- & -- & 25 & \\
\hline 121 & 2802380823422.05 & $\mathrm{~s}$ & 29.0 & 27.0 & 2 & 27.0 & 29.0 & 18.6 & Destroyed 1973 \\
\hline 122 & 2802380823422.04 & $\mathrm{~S}$ & 10.2 & 8.2 & 2 & 8.2 & 10.2 & 18.2 & Destroyed 1973 \\
\hline 123 & 2802380823422.03 & $\mathrm{~F}$ & 48.5 & 48.5 & 2 & -- & -- & 18.3 & Destroyed 1973 \\
\hline 124 & 2802380823049.01 & $\mathrm{~S}$ & 20.2 & 18.2 & $11 / 4$ & 18.2 & 20.2 & 40.2 & \\
\hline 125 & 2802400823059.02 & $\mathrm{~s}$ & 11.8 & 7.8 & 2 & 7.8 & 11.8 & 40 & \\
\hline 126 & 2802400823059.01 & $\mathrm{~s}$ & 24.0 & 20.0 & 2 & 20.0 & 24.0 & 40 & \\
\hline 127 & 2802400823406.01 & $\mathrm{~s}$ & 19.6 & 15.6 & 2 & 15.6 & 19.6 & 25.6 & \\
\hline 128 & 2802400823406.02 & F & 62.5 & 57.5 & 2 & 57.5 & 62.5 & 25.6 & \\
\hline 129 & 2802400823412.02 & $\mathrm{~F}$ & -- & -- & 4 & -- & -- & 22 & \\
\hline 130 & 2802400823412.01 & $\mathrm{~s}$ & 21.0 & 16.0 & 2 & 16.0 & 21.0 & 21.8 & \\
\hline 131 & 2802400823428.01 & $\mathrm{~S}$ & 14.0 & 9.0 & 2 & 9.0 & 14.0 & 24.7 & \\
\hline 132 & 2802400823359.01 & $\mathrm{~s}$ & 18.0 & 16.0 & 2 & 16.0 & 18.0 & 27.3 & \\
\hline 133 & 2802400823359.02 & $\mathrm{~F}$ & 110.0 & 105.0 & 2 & 105.0 & 110.0 & 27.7 & \\
\hline $133 a$ & 2802410823413.01 & $\mathrm{~F}$ & 60.0 & 60.0 & 2 & -- & -- & 18.4 & Destroyed \\
\hline 134 & 2802390823435.03 & $\mathrm{~s}$ & 12.4 & 10.4 & 2 & 10.4 & 12.4 & 17.6 & \\
\hline 135 & 2802390823435.02 & $\mathrm{~s}$ & 21.9 & 19.9 & 2 & 19.9 & 21.9 & 17.8 & \\
\hline 136 & 2802390823435.01 & $\mathrm{~F}$ & 42.9 & 42.0 & 2 & - & -- & 17.6 & \\
\hline 137 & 2802400823426.01 & F & 49.0 & 47.0 & 2 & 47.0 & 49.0 & 23.2 & Destroyed 1972 \\
\hline 138 & 2802400823426.02 & $\mathrm{~s}$ & 23.8 & 21.8 & 2 & 21.8 & 23.8 & 23.2 & \\
\hline 139 & 2802400823426.03 & $\mathrm{~s}$ & 9.2 & 7.2 & 2 & 7.2 & 9.2 & 23.1 & Destroyed 1973 \\
\hline 140 & 2802430823427.01 & $\mathrm{~S}$ & 14.0 & 11.5 & 2 & 11.5 & 14.0 & 25.2 & Destroyed 1972 \\
\hline 141 & 2802430823429.01 & $\mathrm{~s}$ & 14.0 & 11.5 & 2 & 11.5 & 14.0 & 25.0 & Destroyed 1972 \\
\hline 142 & 2802420823435.03 & $\mathrm{~S}$ & 13.4 & 11.4 & 2 & 11.4 & 13.4 & 19.3 & \\
\hline 143 & 2802420823435.02 & $\mathrm{~s}$ & 20.8 & 18.8 & 2 & 18.8 & 20.8 & 19.4 & \\
\hline 144 & 2802420823435.01 & $\mathrm{~F}$ & 49.7 & 49.7 & 2 & -_ & -- & 19.5 & \\
\hline 145 & 2802430823057.03 & $\mathrm{~s}$ & 17.5 & 13.5 & 2 & 13.5 & 17.5 & 36.7 & \\
\hline 146 & 2802430823057.02 & $\mathrm{~s}$ & 33.3 & 29.3 & 2 & 29.3 & 33.3 & 36.8 & \\
\hline 147 & 2802430823057.01 & $\mathrm{~F}$ & 45.8 & 41.8 & 2 & 41.8 & 45.8 & 35.8 & \\
\hline 148 & 2802430823102.02 & S & 13.7 & 9.7 & 2 & 9.7 & 13.7 & 36.9 & \\
\hline 149 & 2802430823102.01 & $\mathrm{~s}$ & 26.5 & 22.5 & 2 & 22.5 & 26.5 & 39.0 & \\
\hline 150 & 2802450823427.01 & $\mathrm{~s}$ & 15.0 & 14.5 & 4 & -- & -- & 26.1 & Destroyed 1972 \\
\hline 151 & 2802450823429.01 & $\mathrm{~s}$ & 14.0 & 11.5 & 2 & 11.5 & 14.0 & 24.8 & Destroyed 1972 \\
\hline 152 & 2802420823426.01 & F & 38.6 & 36.6 & 2 & 36.6 & 38.6 & 24.5 & \\
\hline 153 & 2802420823426.02 & $\mathrm{~s}$ & 14.4 & 12.4 & 2 & 12.4 & 14.4 & 23.8 & \\
\hline 154 & 2802460823432.01 & $\mathrm{~S}$ & 15.0 & 13.0 & 2 & 13.0 & 15.0 & 19.5 & \\
\hline 155 & 2802460823432.02 & $\mathrm{~F}$ & 43.9 & 41.9 & 2 & 41.9 & 43.9 & 19.5 & \\
\hline 156 & 2802460823403.01 & $\mathrm{~F}$ & 79.0 & -- & -- & -- & -- & 25 & \\
\hline 157 & 2802430823425.01 & $\mathrm{~S}$ & 17.0 & 13.0 & 2 & 13.0 & 17.0 & 23.7 & \\
\hline
\end{tabular}


Table 1.--Records of wells in northwest Hillsborough County--Continued

\begin{tabular}{|c|c|c|c|c|c|c|c|c|c|}
\hline \multirow{2}{*}{$\begin{array}{l}\text { We11 } \\
\text { num } \overline{1} \text { ber }\end{array}$} & \multirow{2}{*}{$\begin{array}{c}\text { Identification } \\
\text { number }\end{array}$} & \multirow{2}{*}{$\begin{array}{l}\text { Aquij7 } \\
\text { fer }\end{array}$} & \multirow{2}{*}{$\begin{array}{c}\text { Depth } \\
\text { of } \\
\text { we11- } \\
(f t)\end{array}$} & \multicolumn{2}{|c|}{ Casing } & \multicolumn{2}{|c|}{$\begin{array}{l}\text { Screen } \\
(\mathrm{ft} t)\end{array}$} & \multirow{2}{*}{$\begin{array}{l}\text { Land } \\
\text { surface } \\
\text { alti } \overline{4} / \\
\text { tude } \\
\text { (ft) }\end{array}$} & \multirow{2}{*}{ Remarks } \\
\hline & & & & $\begin{array}{l}\text { Depth } \\
\text { (ft) }\end{array}$ & $\begin{array}{l}\text { Diam- } \\
\text { eter } \\
\text { (in.) }\end{array}$ & From & To & & \\
\hline $\begin{array}{l}158 \\
159\end{array}$ & & $\begin{array}{l}\mathrm{S} \\
\mathrm{F}\end{array}$ & $\begin{array}{l}13.9 \\
39.6\end{array}$ & 11.9 & 2 & $\begin{array}{l}11.9 \\
37.6\end{array}$ & $\begin{array}{l}13.9 \\
39.6\end{array}$ & $\begin{array}{l}23.2 \\
23.4\end{array}$ & Destroyed 1974 \\
\hline $\begin{array}{l}159 \\
160\end{array}$ & & F & $\begin{array}{l}39.6 \\
80.0\end{array}$ & $\begin{array}{l}37.6 \\
--\end{array}$ & $\begin{array}{l}2 \\
2\end{array}$ & $\begin{array}{c}37.6 \\
-\end{array}$ & $\begin{array}{c}39.6 \\
--\end{array}$ & $\begin{array}{l}23.4 \\
25\end{array}$ & Destroyed 1974 \\
\hline 161 & 2802500823418.01 & s & 18.9 & 16.9 & 2 & 16.9 & 18.9 & 21.0 & \\
\hline 162 & 2802470823359.01 & $\mathbf{s}$ & 18.0 & 16.0 & 2 & 16.0 & 18.0 & 26.9 & \\
\hline 163 & 2802490823405.01 & F & 55.0 & -- & 2 & -- & -- & 24.9 & to \\
\hline 164 & 2803160823458.01 & F 1 & 1,120 & 715 & $14-6$ & -- & -- & 24.0 & $\begin{array}{l}\text { St. Petersburg } \\
\text { E-104 }\end{array}$ \\
\hline 165 & 2803170823358.01 & F & -- & -- & 4 & -- & -- & 25 & \\
\hline 166 & 2803540823355.01 & $\mathbf{F}$ & 90 & -- & 3 & -- & -- & 27 & \\
\hline 167 & 2803580823257.01 & F & 60 & 46 & 2 & -- & -- & 35 & \\
\hline 168 & 2804460823025.01 & $\mathbf{F}$ & 556 & 65 & 12 & -- & -- & 58.6 & \\
\hline 169 & 2804470823501.01 & F & 194.0 & 50.0 & 8 & - & -- & 40 & \\
\hline 170 & 2804590823359.01 & $\mathbf{F}$ & -- & -- & 2 & -- & -- & 37 & \\
\hline 171 & 2805040823655.01 & F 1 & 1,200 & 697 & 6 & -- & - & 29.0 & $\begin{array}{l}\text { St. Petersburg } \\
\text { E-102 }\end{array}$ \\
\hline 172 & 2805060823153.01 & $\mathbf{F}$ & -- & -- & 4 & -- & -- & 54 & \\
\hline 173 & 2805070823404.01 & $\mathbf{F}$ & 179 & 40 & 4 & -- & -- & 43.0 & \\
\hline 174 & 2805090823713.01 & F & 280 & 68 & 8 & -- & -- & 25 & \\
\hline 175 & 2805480823557.01 & F 1 & $1,200.0$ & 656.0 & 10 & -- & -- & 41.2 & $\begin{array}{l}\text { St. Petersburg } \\
\text { E-100 }\end{array}$ \\
\hline 176 & 2805500823550.01 & $\mathbf{F}$ & 345.0 & 105.0 & 12 & -- & -- & 43 & Cosme 1C \\
\hline 177 & 2805530823542.01 & $\mathbf{F}$ & 305.0 & 65.0 & 12 & & -- & 43 & Cosme 2 \\
\hline 178 & 2806070823528.01 & F & 335.0 & 128.0 & 12 & - & - & 45 & Cosme $3 \mathrm{~A}$ \\
\hline 179 & 2806010823515.01 & $\mathbf{F}$ & 320.0 & 111.0 & 12 & -- & -- & 44 & Cosme 18 \\
\hline 180 & 2806030823501.01 & $\mathbf{F}$ & 300.0 & 100.0 & 12 & -- & - & 49 & Cosme 16 \\
\hline 181 & 2806100823502.01 & $\mathbf{F}$ & 312.0 & 115.0 & 12 & - & -- & 48 & Cosme 19 \\
\hline 182 & 2806110823515.01 & $\mathbf{F}$ & 345.0 & 65.0 & 12 & -- & -- & 48 & Cosme 4 \\
\hline 183 & 2806120823528.01 & $\mathbf{F}$ & 310.0 & 72 & 12 & - & -- & 48 & Cosme 20 \\
\hline 184 & 2806180823513.01 & $\mathbf{F}$ & 350.0 & 89.0 & 12 & -- & - & 48 & Cosme 5 \\
\hline 185 & 2806220823535.01 & $\mathbf{F}$ & 320.0 & 80.0 & 12 & -- & -- & 48 & Cosme 21 \\
\hline 186 & 2806300823506.01 & $\mathbf{F}$ & 333.0 & 84.0 & 12 & -- & -- & 47 & Cosme 6A \\
\hline 187 & 2806350823501.01 & F & 318.0 & 96.0 & 12 & - & - & 53 & Cosme 9 \\
\hline 188 & 2806410823454.01 & F & 324.0 & 138.0 & 12 & - & -- & 54 & Cosme 7A \\
\hline 189 & 2806490823703.01 & $\mathbf{F}$ & 330.0 & 128.0 & 12 & - & -- & 52 & Cosme 14 \\
\hline 190 & 2806490823449.01 & $\mathbf{F}$ & 345.0 & 82.0 & 12 & -- & -- & 53 & Cosme 8 \\
\hline 191 & 2806520823416.01 & F & 300.0 & 60.0 & 16 & - & - & 53 & Cosme 10 \\
\hline 192 & 2807030823417.01 & $\mathbf{F}$ & 300.0 & 76.0 & 16 & -- & -- & 57.4 & James 11 \\
\hline
\end{tabular}


Table 1.--Records of wells in northwest Hillsborough County--Continued

\begin{tabular}{|c|c|c|c|c|c|c|c|c|c|}
\hline \multirow{2}{*}{$\begin{array}{l}\text { Well } \\
\text { num }_{\overline{1}} / \\
\text { ber }^{-}\end{array}$} & \multirow{2}{*}{$\begin{array}{c}\text { Identification } \\
\text { number }\end{array}$} & \multirow{2}{*}{$\begin{array}{l}\text { Aquij } \\
\text { fer }\end{array}$} & \multirow{2}{*}{$\begin{array}{c}\text { Depth } \\
\text { of } \\
\text { wel1 } \frac{3}{} \\
\text { (ft) }\end{array}$} & \multicolumn{2}{|c|}{ Casing } & \multicolumn{2}{|c|}{$\begin{array}{l}\text { Screen } \\
(\mathrm{f} t)\end{array}$} & \multirow{2}{*}{$\begin{array}{l}\text { Land } \\
\text { surface } \\
\text { alti } \overline{4} / \\
\text { tude } \\
\text { (ft) }\end{array}$} & \multirow{2}{*}{ Remarks } \\
\hline & & & & $\begin{array}{l}\text { Depth }{ }^{3 /} \\
\text { (ft) }\end{array}$ & $\begin{array}{l}\text { Diam- } \\
\text { eter } \\
\text { (in.) }\end{array}$ & From & To & & \\
\hline 193 & 2807120823415.01 & $\mathbf{F}$ & 300.0 & 88.0 & 12 & -- & -- & 56 & Cosme $12 \mathrm{~A}$ \\
\hline 194 & 2807170823421.01 & $\mathbf{F}$ & 354.0 & 98.0 & 12 & -- & -- & 50 & Cosme 23 \\
\hline 195 & 2807250823422.01 & $\mathrm{~F}$ & 357.0 & 98.0 & 12 & -- & -- & 56 & Cosme 24 \\
\hline 196 & 2807320823428.01 & F & 350.0 & 98.0 & 12 & -- & -- & 56 & Cosme 25 \\
\hline 197 & 2808130823430.01 & F & 384.0 & 93.0 & 12 & -- & -- & 61 & Cosme 30 \\
\hline 198 & 2808200823431.01 & $\mathbf{F}$ & 374.0 & 102.0 & 12 & -- & -- & 62 & Cosme 31 \\
\hline 199 & 2808270823433.01 & F & 300.0 & 84.0 & 12 & - & -- & 58 & Cosme 32 \\
\hline 200 & 2808330823436.01 & $\mathbf{F}$ & 407.0 & 102.0 & -- & -- & -- & 56 & Cosme 33A \\
\hline 201 & 2808400823438.01 & $\mathbf{F}$ & 407.0 & 100.0 & 12 & -- & -- & 62 & Cosme 34 \\
\hline 202 & 2807100823256.01 & F & 46.0 & 42.0 & 2 & 42.0 & 46.0 & 51.4 & \\
\hline 203 & 2807190823229.01 & $\mathbf{F}$ & 62.0 & 58.0 & 2 & 58.0 & 62.0 & 56.0 & \\
\hline 204 & 2808030823239.01 & $\mathbf{F}$ & 70.0 & -- & 2 & - & -- & & Hills. Dairy \\
\hline 205 & 2806460823202.01 & $\mathbf{F}$ & 60.0 & 56.0 & 2 & 56.0 & 60.0 & 57.7 & \\
\hline 206 & 2807120823202.01 & F & 59.0 & 55.0 & 2 & 55.0 & 59.0 & 58.3 & \\
\hline 207 & 2807410823202.01 & $\mathbf{F}$ & 55.0 & 51.0 & 2 & 51.0 & 55.0 & 56.3 & \\
\hline 208 & 2806570823058.01 & $\mathbf{F}$ & 411.0 & 71.0 & 20 & -- & -- & 55 & Section 21-3 \\
\hline 209 & 2807080823058.01 & $\mathbf{F}$ & 412.0 & 73.0 & 20 & -- & -- & 57 & Section 21-2 \\
\hline 210 & 2806560823058.01 & F & 570.0 & 70.0 & 20 & -- & -- & 57 & Section $21-1$ \\
\hline 211 & 2807320823058.01 & $\mathbf{F}$ & 300.0 & 141.0 & - & -- & -- & -- & Section 21-26A \\
\hline 212 & 2807380823047.01 & $\mathbf{F}$ & 601.0 & 71.0 & 20 & -- & -- & 54 & Section 21-4 \\
\hline 213 & 2807380823032.01 & $\mathbf{F}$ & 601.0 & 75.0 & 20 & -- & - & 57 & Section $21-5$ \\
\hline 214 & 2807380823020.01 & F & 412.0 & 79.0 & 20 & - & -- & 58 & Section $21-6$ \\
\hline 215 & 2807280823011.01 & F 1 & $1,250.0$ & 718.0 & 6 & -- & -- & 56 & Section 21-7 \\
\hline 216 & 2807170823010.01 & F & 551.0 & 116.0 & 16 & -- & -- & 55 & Section 21-8 \\
\hline 217 & 2807080823010.01 & F & 601.0 & 79.0 & 20 & - & -- & 56 & Section 21-9 \\
\hline 218 & 2806480823011.01 & $\mathbf{F}$ & 411.0 & 70.0 & 20 & -- & -- & 56 & Section $21-10$ \\
\hline 219 & 2807020823028.01 & F & 347.0 & 46.0 & 12 & -- & -- & 56.8 & Section $21-13$ \\
\hline
\end{tabular}

1/ Location of wells shown in figures 2 and 3.

2/ F, Floridan aquifer; $S$, surficial aquifer.

3/ Depths shown to nearest tenth of a foot are measured; other depths are reported.

4/ Altitudes shown to nearest tenth of a foot above National Geodetic Vertical Datum of 1929, determined by level instrument; other altitudes estimated from topographic maps. 
Table 2.--Water-1eve1 measurements in wells in northwest

Hillsborough County

\begin{tabular}{|c|c|c|c|c|c|}
\hline \multirow[b]{2}{*}{$\begin{array}{l}\text { Well } 1 / \\
\text { number }\end{array}$} & \multirow[b]{2}{*}{ Aquifer $2 /$} & \multirow{2}{*}{$\begin{array}{l}\text { Depth } \\
\text { of } \\
\text { wel1 } 3 / \\
\text { (ft) }\end{array}$} & \multirow[b]{2}{*}{$\begin{array}{c}\text { Date of } \\
\text { measurement }\end{array}$} & \multicolumn{2}{|c|}{$\begin{array}{l}\text { Water level } \\
(\mathrm{ft})\end{array}$} \\
\hline & & & & $\begin{array}{l}\text { Above ( }(+) \\
\text { or below } \\
\text { land } \\
\text { surface }\end{array}$ & Altitude $e^{4 /}$ \\
\hline 8 & S & 16.0 & $\begin{array}{r}11-10-70 \\
5-14-71 \\
10-28-71 \\
5-24-72\end{array}$ & $\begin{array}{l}3.6 \\
4.2 \\
2.9 \\
4.2\end{array}$ & $\begin{array}{l}3 \\
3 \\
4 \\
3\end{array}$ \\
\hline 19 & $\mathbf{F}$ & 40.0 & $\begin{array}{r}11-10-70 \\
5-14-71 \\
10-28-71 \\
5-24-72 \\
10-16-72 \\
5-17-73 \\
10-04-73\end{array}$ & $\begin{array}{l}12.4 \\
13.2 \\
11.6 \\
12.9 \\
12.3 \\
12.6 \\
11.6\end{array}$ & $\begin{array}{l}12 \\
11 \\
12 \\
11 \\
12 \\
11 \\
12\end{array}$ \\
\hline 26 & $\mathbf{S}$ & 18.0 & $\begin{array}{r}11-10-70 \\
5-14-71 \\
10-28-71 \\
5-24-72 \\
5-16-73\end{array}$ & $\begin{array}{l}2.7 \\
3.6 \\
1.0 \\
3.6 \\
3.0\end{array}$ & $\begin{array}{l}5 \\
4 \\
7 \\
4 \\
5\end{array}$ \\
\hline 27 & $\mathbf{F}$ & 50.0 & $\begin{array}{r}11-10-70 \\
5-14-71 \\
10-28-71 \\
5-24-72 \\
9-22-72 \\
5-16-73 \\
10-04-73\end{array}$ & $\begin{array}{r}.7 \\
2.1 \\
.8 \\
1.9 \\
1.3 \\
2.0 \\
1.0\end{array}$ & $\begin{array}{l}7 \\
6 \\
7 \\
6 \\
7 \\
6 \\
7\end{array}$ \\
\hline 29 & $\mathbf{S}$ & 19.8 & $3-04-69$ & 2.5 & 12 \\
\hline 30 & $\mathbf{F}$ & 39.2 & $3-04-69$ & 3.7 & 11 \\
\hline 31 & $\mathbf{F}$ & 330.0 & $\begin{array}{r}12-19-68 \\
11-10-70 \\
10-28-71 \\
5-24-72 \\
10-16-72 \\
5-16-73 \\
10-04-73\end{array}$ & $\begin{array}{l}1.0 \\
4.6 \\
1.4 \\
2.7 \\
2.1 \\
2.5 \\
1.5\end{array}$ & $\begin{array}{l}7.9 \\
4.3 \\
7.5 \\
6.2 \\
6.8 \\
6.4 \\
7.4\end{array}$ \\
\hline 34 & F & 150 & $\begin{array}{r}11-10-70 \\
5-14-71 \\
10-28-71 \\
5-24-72 \\
10-16-72 \\
5-16-73 \\
10-04-73\end{array}$ & $\begin{array}{r}12.4 \\
14.1 \\
9.6 \\
13.1 \\
11.8 \\
14.6 \\
11.1\end{array}$ & $\begin{array}{l}23 \\
21 \\
25 \\
22 \\
23 \\
20 \\
24\end{array}$ \\
\hline
\end{tabular}

Footnotes are at end of table. 
Table 2.--Water-1evel measurements in wells in northwest Hillsborough County--Continued

\begin{tabular}{|c|c|c|c|c|c|}
\hline \multirow[b]{2}{*}{ Well $_{\text {number }} 1 /$} & \multirow[b]{2}{*}{ Aquifer $2 /$} & \multirow{2}{*}{$\begin{array}{l}\text { Depth } \\
\text { of } \\
\text { we11 } 3 / \\
(\mathrm{ft})\end{array}$} & \multirow[b]{2}{*}{$\begin{array}{c}\text { Date of } \\
\text { measurement }\end{array}$} & \multicolumn{2}{|c|}{$\begin{array}{l}\text { Water level } \\
\text { (ft) }\end{array}$} \\
\hline & & & & $\begin{array}{l}\text { Above }(+) \\
\text { or below } \\
\text { land } \\
\text { surface }\end{array}$ & Altitude ${ }^{4 /}$ \\
\hline 35 & S & 9.3 & $\begin{array}{r}11-10-70 \\
5-14-71 \\
10-28-71 \\
5-24-72\end{array}$ & $\begin{array}{l}8.1 \\
8.4 \\
5.7 \\
7.8\end{array}$ & $\begin{array}{l}14.1 \\
13.8 \\
16.5 \\
14.4\end{array}$ \\
\hline 36 & F & 90.0 & $\begin{array}{r}5-14-71 \\
10-28-71 \\
5-24-72 \\
10-16-72 \\
5-16-73 \\
10-04-73\end{array}$ & $\begin{array}{l}4.9 \\
3.3 \\
4.4 \\
4.5 \\
4.4 \\
3.5\end{array}$ & $\begin{array}{l}5.2 \\
6.8 \\
5.7 \\
5.6 \\
5.7 \\
6.6\end{array}$ \\
\hline 38 & $\mathbf{F}$ & 260 & $\begin{array}{r}4-09-73 \\
5-16-73 \\
10-04-73\end{array}$ & $\begin{array}{l}4.0 \\
6.8 \\
5.2\end{array}$ & $\begin{array}{l}19.3 \\
16.5 \\
18.1\end{array}$ \\
\hline 39 & $\mathbf{F}$ & -- & $\begin{array}{r}11-10-70 \\
5-14-71 \\
10-28-71 \\
5-24-72 \\
10-04-73\end{array}$ & $\begin{array}{r}+2.0 \\
+\quad .8 \\
\text { Flowing } \\
\text { Flowing } \\
\text { Flowing }\end{array}$ & $\begin{array}{r}7 \\
6 \\
-- \\
-- \\
--\end{array}$ \\
\hline 40 & $\mathbf{F}$ & 405.0 & $4-24-73$ & 10.7 & 17.9 \\
\hline 41 & $\mathbf{F}$ & 180.0 & $\begin{array}{r}8-30-72 \\
10-16-72\end{array}$ & $\begin{array}{l}2.8 \\
3.8\end{array}$ & $\begin{array}{l}14 \\
13\end{array}$ \\
\hline 42 & $\mathbf{F}$ & 174.0 & $\begin{array}{r}9-05-72 \\
10-16-72\end{array}$ & $\begin{array}{l}1.1 \\
1.6\end{array}$ & $\begin{array}{l}14 \\
.13\end{array}$ \\
\hline 43 & $\mathbf{F}$ & 94 & $\begin{array}{r}8-19-71 \\
10-16-72 \\
5-16-73 \\
10-04-73\end{array}$ & $\begin{array}{l}2.3 \\
3.2 \\
4.7 \\
2.8\end{array}$ & $\begin{array}{l}14 \\
13 \\
11 \\
13\end{array}$ \\
\hline 46 & $\mathrm{~S}$ & 21.3 & $10-06-70$ & 1.8 & 8 \\
\hline 47 & $\mathbf{S}$ & 15.6 & $10-02-70$ & 2.4 & 8 \\
\hline 48 & $S$ & 21.6 & $10-02-70$ & 1.8 & 8 \\
\hline 49 & $\mathbf{S}$ & 18.0 & $\begin{array}{r}5-12-71 \\
10-28-71 \\
9-22-72 \\
5-11-73 \\
10-03-73\end{array}$ & $\begin{array}{l}6.2 \\
5.4 \\
6.6 \\
6.6 \\
5.7\end{array}$ & $\begin{array}{l}17.8 \\
18.3 \\
17.4 \\
17.4 \\
18.3\end{array}$ \\
\hline
\end{tabular}


Table 2.--Water-1eve1 measurements in wells in northwest

Hillsborough County--Continued

\begin{tabular}{|c|c|c|c|c|c|}
\hline \multirow[b]{2}{*}{$\begin{array}{c}\text { We11 } 1 / \\
\text { number }\end{array}$} & \multirow[b]{2}{*}{ Aquifer $2 /$} & \multirow{2}{*}{$\begin{array}{l}\text { Depth } \\
\text { of } \\
\text { we11 } 3 / \\
(f t)\end{array}$} & \multirow[b]{2}{*}{$\begin{array}{l}\text { Date of } \\
\text { measurement }\end{array}$} & \multicolumn{2}{|c|}{$\begin{array}{l}\text { Water level } \\
\text { (ft) }\end{array}$} \\
\hline & & & & $\begin{array}{c}\text { Above (t) } \\
\text { or below } \\
\text { land } \\
\text { surface }\end{array}$ & Altitude $4 /$ \\
\hline 50 & F & 46.5 & $\begin{array}{r}5-12-71 \\
10-28-71 \\
9-22-72 \\
5-11-73 \\
10-03-73\end{array}$ & $\begin{array}{l}7.3 \\
5.5 \\
4.8 \\
4.3 \\
4.7\end{array}$ & $\begin{array}{l}16.9 \\
18.7 \\
19.4 \\
19.9 \\
19.5\end{array}$ \\
\hline 51 & $\mathbf{F}$ & - & $\begin{array}{r}11-10-70 \\
5-13-71 \\
10-28-71\end{array}$ & $\begin{array}{l}2.0 \\
3.2 \\
1.1\end{array}$ & $\begin{array}{l}13 \\
12 \\
14\end{array}$ \\
\hline 52 & $\mathbf{F}$ & 200 & $1-29-70$ & +1.5 & 17 \\
\hline 54 & F & -- & $\begin{array}{r}11-09-70 \\
5-13-71 \\
10-28-71 \\
5-24-72 \\
10-17-72 \\
5-18-73 \\
10-04-73\end{array}$ & $\begin{array}{l}5.4 \\
6.5 \\
2.8 \\
4.7 \\
5.0 \\
5.0 \\
6.0\end{array}$ & $\begin{array}{l}22 \\
21 \\
24 \\
22 \\
22 \\
22 \\
21\end{array}$ \\
\hline 55 & $\mathbf{S}$ & 10.0 & $\begin{array}{r}11-09-70 \\
5-13-71 \\
10-28-71 \\
5-24-72 \\
10-16-72 \\
5-18-73 \\
10-04-73\end{array}$ & $\begin{array}{r}3.2 \\
3.4 \\
.3 \\
2.3 \\
2.1 \\
3.2 \\
.8\end{array}$ & $\begin{array}{l}29.2 \\
29.0 \\
32.1 \\
30.1 \\
30.3 \\
29.2 \\
31.6\end{array}$ \\
\hline 56 & $\mathbf{F}$ & 42.8 & $\begin{array}{r}11-09-70 \\
5-13-71 \\
10-28-71 \\
5-24-72 \\
10-16-72 \\
5-18-73 \\
10-04-73\end{array}$ & $\begin{array}{r}7.4 \\
10.8 \\
4.8 \\
7.9 \\
9.7 \\
9.6 \\
6.7\end{array}$ & $\begin{array}{l}24.9 \\
21.5 \\
27.5 \\
24.4 \\
22.6 \\
22.7 \\
25.6\end{array}$ \\
\hline 57 & F & 37.0 & $\begin{array}{r}11-09-70 \\
5-13-71 \\
10-28-71 \\
10-16-72 \\
5-18-73 \\
10-04-73\end{array}$ & $\begin{array}{r}11.3 \\
14.9 \\
8.8 \\
12.5 \\
12.3 \\
9.9\end{array}$ & $\begin{array}{l}25.0 \\
21.4 \\
27.5 \\
23.8 \\
24.0 \\
26.4\end{array}$ \\
\hline
\end{tabular}


Table 2.--Water-level measurements in wells in northwest Hillsborough County--Continued

\begin{tabular}{|c|c|c|c|c|c|}
\hline \multirow[b]{2}{*}{ We11 $1 /$} & \multirow[b]{2}{*}{ Aquifer $2 /$} & \multirow{2}{*}{$\begin{array}{l}\text { Depth } \\
\text { of } 3 / \\
\text { we11 }- \\
(f t)\end{array}$} & \multirow[b]{2}{*}{$\begin{array}{c}\text { Date of } \\
\text { measurement }\end{array}$} & \multicolumn{2}{|c|}{$\begin{array}{l}\text { Water level } \\
(\mathrm{ft})\end{array}$} \\
\hline & & & & $\begin{array}{c}\text { Above (t) } \\
\text { or below } \\
\text { land } \\
\text { surface }\end{array}$ & Altitude $4 /$ \\
\hline 58 & $S$ & 15.0 & $\begin{array}{r}11-09-70 \\
5-13-71 \\
10-28-71 \\
5-24-72 \\
10-16-72 \\
5-18-73 \\
10-04-73\end{array}$ & $\begin{array}{l}5.7 \\
6.0 \\
2.6 \\
5.3 \\
4.0 \\
4.6 \\
1.7\end{array}$ & $\begin{array}{l}29.9 \\
29.6 \\
33.0 \\
30.3 \\
31.6 \\
31.0 \\
33.8\end{array}$ \\
\hline 59 & $\mathrm{~S}$ & 8.8 & $\begin{array}{r}11-09-70 \\
5-13-71 \\
10-28-71 \\
5-24-72 \\
10-16-72\end{array}$ & $\begin{array}{l}5.5 \\
5.3 \\
3.1 \\
4.9 \\
3.8\end{array}$ & $\begin{array}{l}27.9 \\
28.1 \\
30.3 \\
28.5 \\
29.6\end{array}$ \\
\hline 60 & S & 9.2 & $\begin{array}{l}11-09-70 \\
10-28-71\end{array}$ & $\begin{array}{l}4.4 \\
2.0\end{array}$ & $\begin{array}{l}29.9 \\
32.3\end{array}$ \\
\hline 61 & $S$ & 8.5 & $\begin{array}{r}11-09-70 \\
5-13-71 \\
10-28-71\end{array}$ & $\begin{array}{l}4.9 \\
5.0 \\
2.5\end{array}$ & $\begin{array}{l}23.7 \\
23.6 \\
26.1\end{array}$ \\
\hline 62 & $S$ & 9.0 & $\begin{array}{r}11-10-70 \\
5-13-71 \\
10-28-71 \\
5-24-72\end{array}$ & $\begin{array}{l}7.3 \\
7.8 \\
2.9 \\
6.6\end{array}$ & $\begin{array}{r}9.4 \\
8.9 \\
13.8 \\
10.1\end{array}$ \\
\hline 63 & $S$ & 9.0 & $\begin{array}{r}11-09-70 \\
5-12-71 \\
10-29-71 \\
5-16-72 \\
9-22-72 \\
5-11-73 \\
10-03-73\end{array}$ & $\begin{array}{r}4.6 \\
5.2 \\
.8 \\
4.0 \\
3.5 \\
3.9 \\
1.5\end{array}$ & $\begin{array}{l}17.9 \\
17.3 \\
21.7 \\
18.5 \\
19.0 \\
18.6 \\
21.0\end{array}$ \\
\hline 65 & S & 12.0 & $\begin{array}{r}11-09-70 \\
5-12-71 \\
10-29-71 \\
5-16-72 \\
9-22-72 \\
5-11-73 \\
10-03-73\end{array}$ & $\begin{array}{l}6.0 \\
6.1 \\
2.6 \\
5.7 \\
6.0 \\
5.3 \\
3.4\end{array}$ & $\begin{array}{l}13.6 \\
13.5 \\
17.0 \\
13.9 \\
13.6 \\
14.3 \\
16.2\end{array}$ \\
\hline
\end{tabular}


Table 2.--Water-1eve1 measurements in we11s in northwest Hillsborough County--Continued

\begin{tabular}{|c|c|c|c|c|c|}
\hline \multirow[b]{2}{*}{$\begin{array}{l}\text { Well } 1 / \\
\text { number }\end{array}$} & \multirow[b]{2}{*}{ Aquifer $2 /$} & \multirow{2}{*}{$\begin{array}{l}\text { Depth } \\
\text { of } \\
\text { wel1 } 3 / \\
(f t)\end{array}$} & \multirow[b]{2}{*}{$\begin{array}{c}\text { Date of } \\
\text { measurement }\end{array}$} & \multicolumn{2}{|c|}{$\begin{array}{l}\text { Water level } \\
(\mathrm{ft})\end{array}$} \\
\hline & & & & $\begin{array}{l}\text { Above }(+) \\
\text { or below } \\
\text { land } \\
\text { surface }\end{array}$ & Altitude $\mathrm{4}^{4 /}$ \\
\hline 66 & $\mathrm{~S}$ & 9.6 & $\begin{array}{r}12-30-69 \\
11-09-70 \\
5-12-71 \\
10-28-71 \\
5-16-72 \\
9-22-72 \\
5-11-73 \\
10-03-73\end{array}$ & $\begin{array}{l}2.3 \\
5.6 \\
5.6 \\
2.5 \\
5.2 \\
5.2 \\
4.8 \\
2.9\end{array}$ & $\begin{array}{l}14.5 \\
11.2 \\
11.2 \\
14.3 \\
11.6 \\
11.6 \\
12.0 \\
13.9\end{array}$ \\
\hline 67 & $\mathrm{~F}$ & 40.3 & $\begin{array}{r}12-30-69 \\
11-09-70 \\
5-12-71 \\
10-28-71 \\
5-16-72 \\
9-22-72 \\
5-11-73 \\
10-03-73\end{array}$ & $\begin{array}{r}+2.4 \\
+\quad .8 \\
+1.5 \\
+1.8 \\
+\quad .4 \\
+\quad .5 \\
+\quad .2 \\
+1.1\end{array}$ & $\begin{array}{l}19.5 \\
17.9 \\
15.6 \\
18.9 \\
17.5 \\
17.6 \\
17.3 \\
18.2\end{array}$ \\
\hline 68 & $\mathrm{~S}$ & 10.0 & $7-31-69$ & 2.4 & 12.7 \\
\hline 69 & $\mathrm{~s}$ & 15.7 & $\begin{array}{r}5-11-73 \\
10-03-73\end{array}$ & $\begin{array}{l}6.5 \\
4.6\end{array}$ & $\begin{array}{l}15.8 \\
17.7\end{array}$ \\
\hline 70 & $\mathrm{~F}$ & 65.5 & $\begin{array}{r}5-11-73 \\
10-03-73\end{array}$ & $\begin{array}{l}4.7 \\
5.9\end{array}$ & $\begin{array}{l}17.7 \\
16.5\end{array}$ \\
\hline 72 & $\mathrm{~F}$ & 47.0 & $\begin{array}{r}8-08-69 \\
11-09-70 \\
5-12-71 \\
10-29-71 \\
5-16-72\end{array}$ & $\begin{array}{r}+1.6 \\
+1.5 \\
+2.0 \\
+1.0\end{array}$ & $\begin{array}{l}16.7 \\
16.6 \\
15.1 \\
17.1 \\
16.1\end{array}$ \\
\hline 73 & $\mathrm{~F}$ & 67.0 & $\begin{array}{r}5-12-71 \\
10-28-71 \\
5-16-72 \\
9-22-72 \\
5-11-73 \\
10-03-73\end{array}$ & $\begin{array}{l}5.9 \\
3.7 \\
4.2 \\
5.5 \\
5.5 \\
3.9\end{array}$ & $\begin{array}{l}18.5 \\
20.7 \\
20.2 \\
18.9 \\
18.9 \\
20.5\end{array}$ \\
\hline 74 & $\mathrm{~S}$ & 18.0 & $\begin{array}{r}5-12-71 \\
10-28-71 \\
5-16-72 \\
9-22-72 \\
5-11-73 \\
10-03-73\end{array}$ & $\begin{array}{l}5.4 \\
1.2 \\
3.9 \\
4.8 \\
4.2 \\
2.1\end{array}$ & $\begin{array}{l}18.9 \\
23.1 \\
20.4 \\
19.5 \\
20.1 \\
22.2\end{array}$ \\
\hline
\end{tabular}


Table 2.--Water-level measurements in wells in northwest

\section{Hillsborough County--Continued}

\begin{tabular}{|c|c|c|c|c|c|}
\hline \multirow[b]{2}{*}{$\begin{array}{l}\text { We11 } 1 / \\
\text { number }\end{array}$} & \multirow[b]{2}{*}{ Aquifer $2 /$} & \multirow{2}{*}{$\begin{array}{l}\text { Depth } \\
\text { of } \\
\text { wel1 } 3 / \\
(f t)\end{array}$} & \multirow[b]{2}{*}{$\begin{array}{c}\text { Date of } \\
\text { measurement }\end{array}$} & \multicolumn{2}{|c|}{$\begin{array}{l}\text { Water level } \\
(\mathrm{ft})\end{array}$} \\
\hline & & & & $\begin{array}{l}\text { Above ( }+ \text { ) } \\
\text { or below } \\
\text { land } \\
\text { surface }\end{array}$ & Altitude 4/ $^{\text {/ }}$ \\
\hline 75 & $\mathrm{~S}$ & 14.9 & $\begin{array}{r}5-11-73 \\
10-03-73\end{array}$ & $\begin{array}{l}6.5 \\
5.2\end{array}$ & $\begin{array}{l}15.5 \\
16.8\end{array}$ \\
\hline 76 & $\mathrm{~F}$ & 80.0 & $\begin{array}{r}10-28-71 \\
9-22-72 \\
10-03-73\end{array}$ & $\begin{array}{l}2.8 \\
2.8 \\
2.7\end{array}$ & $\begin{array}{l}16.6 \\
16.6 \\
16.7\end{array}$ \\
\hline 77 & $\mathrm{~S}$ & 9.3 & $\begin{array}{r}12-31-69 \\
11-09-70 \\
10-28-71 \\
5-16-72 \\
9-22-72 \\
5-11-73 \\
10-03-73\end{array}$ & $\begin{array}{l}6.4 \\
7.8 \\
6.8 \\
\text { dry } \\
7.7 \\
7.4 \\
6.7\end{array}$ & $\begin{array}{r}12.9 \\
11.5 \\
12.5 \\
\text { dry } \\
11.6 \\
11.9 \\
12.6\end{array}$ \\
\hline 78 & S & 24.4 & $\begin{array}{r}1-23-70 \\
11-09-70 \\
5-12-71 \\
10-28-71 \\
5-16-72 \\
9-22-72 \\
5-11-73 \\
10-03-73\end{array}$ & $\begin{array}{l}6.4 \\
7.3 \\
7.4 \\
6.2 \\
7.2 \\
7.1 \\
6.9 \\
6.1\end{array}$ & $\begin{array}{l}12.4 \\
11.5 \\
11.4 \\
12.6 \\
11.6 \\
11.7 \\
11.9 \\
12.7\end{array}$ \\
\hline 79 & S & 14.0 & $\begin{array}{r}6-16-71 \\
10-29-71 \\
5-16-72 \\
9-22-72 \\
5-11-73 \\
10-03-73\end{array}$ & $\begin{array}{r}11.4 \\
10.0 \\
10.8 \\
8.5 \\
10.2 \\
9.6\end{array}$ & $\begin{array}{l}10.4 \\
11.8 \\
11.0 \\
13.3 \\
11.6 \\
12.2\end{array}$ \\
\hline 80 & S & 13.0 & $\begin{array}{r}10-29-71 \\
5-16-72 \\
9-22-72 \\
5-11-73 \\
10-03-73\end{array}$ & $\begin{array}{l}11.2 \\
11.2 \\
11.9 \\
11.7 \\
11.4\end{array}$ & $\begin{array}{l}10.9 \\
10.9 \\
10.2 \\
10.4 \\
10.7\end{array}$ \\
\hline 82 & $\mathrm{~S}$ & 19.5 & $\begin{array}{r}5-11-73 \\
10-03-73\end{array}$ & $\begin{array}{l}5.7 \\
3.7\end{array}$ & $\begin{array}{l}19.3 \\
21.3\end{array}$ \\
\hline 83 & $S$ & 13.9 & $\begin{array}{r}1-23-70 \\
11-09-70 \\
5-12-71 \\
10-29-71 \\
5-16-72 \\
9-22-72 \\
5-11-73 \\
10-03-73\end{array}$ & $\begin{array}{r}8.2 \\
10.0 \\
10.0 \\
7.8 \\
9.1 \\
9.2 \\
9.3 \\
8.2\end{array}$ & $\begin{array}{l}8.7 \\
6.9 \\
6.9 \\
9.1 \\
7.8 \\
7.7 \\
7.6 \\
8.7\end{array}$ \\
\hline
\end{tabular}


Table 2.--Water-1eve1 measurements in we1ls in northwest Hillsborough County--Continued

\begin{tabular}{|c|c|c|c|c|c|}
\hline \multirow[b]{2}{*}{$\begin{array}{l}\text { Well } 1 / \\
\text { number }\end{array}$} & \multirow[b]{2}{*}{ Aquifer $2 /$} & \multirow{2}{*}{$\begin{array}{l}\text { Depth } \\
\text { of } 3 / \\
\text { wel1 }- \\
\text { (ft) }\end{array}$} & \multirow[b]{2}{*}{$\begin{array}{c}\text { Date of } \\
\text { measurement }\end{array}$} & \multicolumn{2}{|c|}{$\begin{array}{l}\text { Water level } \\
\text { (ft) }\end{array}$} \\
\hline & & & & $\begin{array}{l}\text { Above }(+) \\
\text { or below } \\
\text { land } \\
\text { surface }\end{array}$ & Altitude $4 /$ \\
\hline 84 & $\mathbf{S}$ & 24.0 & $\begin{array}{r}11-09-70 \\
5-12-71 \\
10-29-71 \\
5-16-72 \\
9-22-72 \\
5-11-73 \\
10-03-73\end{array}$ & $\begin{array}{r}10.1 \\
10.3 \\
8.1 \\
9.1 \\
8.7 \\
9.1 \\
8.1\end{array}$ & $\begin{array}{l}7.1 \\
6.9 \\
9.1 \\
8.1 \\
8.5 \\
8.1 \\
9.1\end{array}$ \\
\hline 85 & $\mathbf{F}$ & 50.5 & $\begin{array}{r}2-04-70 \\
11-09-70 \\
5-12-71 \\
10-29-71 \\
5-16-72 \\
9-22-72 \\
5-11-73\end{array}$ & $\begin{array}{r}+2.0 \\
+\quad .4 \\
1.5 \\
+1.5 \\
+\quad .3 \\
.1 \\
.3\end{array}$ & $\begin{array}{l}18.8 \\
17.2 \\
15.3 \\
18.3 \\
17.1 \\
16.7 \\
16.5\end{array}$ \\
\hline 86 & $\mathbf{S}$ & 23.0 & $\begin{array}{r}11-09-70 \\
5-12-71 \\
10-29-71 \\
5-16-72 \\
9-22-72 \\
5-11-73 \\
10-03-73\end{array}$ & $\begin{array}{l}8.4 \\
8.4 \\
6.7 \\
7.2 \\
6.8 \\
6.4 \\
5.3\end{array}$ & $\begin{array}{l}12.6 \\
12.6 \\
14.3 \\
13.8 \\
14.2 \\
14.6 \\
15.7\end{array}$ \\
\hline 87 & $\mathbf{S}$ & 8.3 & $\begin{array}{r}12-31-69 \\
5-12-71 \\
10-29-71 \\
5-16-72 \\
9-22-72 \\
5-11-73 \\
10-03-73\end{array}$ & $\begin{array}{l}3.0 \\
7.9 \\
6.1 \\
6.8 \\
6.3 \\
5.8 \\
4.8\end{array}$ & $\begin{array}{l}17.7 \\
12.8 \\
14.6 \\
13.9 \\
14.4 \\
14.9 \\
15.9\end{array}$ \\
\hline 88 & $\mathbf{F}$ & 65.4 & $\begin{array}{r}5-11-73 \\
10-03-73\end{array}$ & $\begin{array}{l}6.8 \\
6.1\end{array}$ & $\begin{array}{l}18.1 \\
18.7\end{array}$ \\
\hline 89 & 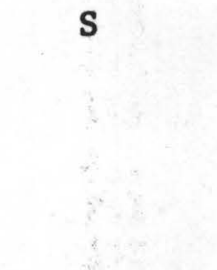 & 14.6 & $\begin{array}{r}11-09-70 \\
5-13-71 \\
10-28-71 \\
5-16-72 \\
5-11-73 \\
10-03-73\end{array}$ & $\begin{array}{r}.7 \\
1.9 \\
1.8 \\
4.2 \\
5.9 \\
4.7\end{array}$ & $\begin{array}{l}24.1 \\
22.9 \\
23.0 \\
20.6 \\
18.9 \\
20.1\end{array}$ \\
\hline 90 & $\mathbf{S}$ & 14.0 & $\begin{array}{r}6-06-71 \\
10-29-71 \\
5-16-72 \\
9-22-72 \\
5-11-73 \\
10-03-73\end{array}$ & $\begin{array}{r}11.1 \\
8.8 \\
10.2 \\
9.2 \\
8.2 \\
7.0\end{array}$ & $\begin{array}{l}12.9 \\
15.2 \\
13.8 \\
14.8 \\
15.8 \\
17.0\end{array}$ \\
\hline & & & 18 & 5 & \\
\hline
\end{tabular}


Table 2.--Water-level measurements in wells in northwest Hillsborough County--Continued

\begin{tabular}{|c|c|c|c|c|c|}
\hline \multirow[b]{2}{*}{$\underset{\text { number }}{\text { Well }}$} & \multirow[b]{2}{*}{ Aquifer $2 /$} & \multirow{2}{*}{$\begin{array}{l}\text { Depth } \\
\text { of } 3 / \\
\text { wel1 } / \\
(\mathrm{ft})\end{array}$} & \multirow[b]{2}{*}{$\begin{array}{c}\text { Date of } \\
\text { measurement }\end{array}$} & \multicolumn{2}{|c|}{$\begin{array}{l}\text { Water level } \\
\text { (ft) }\end{array}$} \\
\hline & & & & $\begin{array}{c}\text { Above }(t) \\
\text { or below } \\
\text { land } \\
\text { surface }\end{array}$ & Altitude $e^{4 /}$ \\
\hline 91 & $\mathbf{s}$ & 14.5 & $\begin{array}{r}6-16-71 \\
10-29-71 \\
5-16-72 \\
9-22-72 \\
5-11-73 \\
10-03-73\end{array}$ & $\begin{array}{l}13.0 \\
10.4 \\
\text { dry } \\
11.6 \\
10.8 \\
9.2\end{array}$ & $\begin{array}{l}11.1 \\
13.7 \\
\text { dry } \\
12.5 \\
13.3 \\
14.9\end{array}$ \\
\hline 92 & $\mathbf{S}$ & 11.5 & $\begin{array}{r}11-09-70 \\
5-12-71 \\
10-29-71 \\
5-16-72 \\
9-22-72 \\
5-11-73 \\
10-03-73\end{array}$ & $\begin{array}{l}4.0 \\
4.2 \\
3.7 \\
4.1 \\
3.9 \\
4.0 \\
3.5\end{array}$ & $\begin{array}{l}5.7 \\
5.5 \\
6.0 \\
5.6 \\
5.8 \\
5.7 \\
6.2\end{array}$ \\
\hline 93 & $\mathbf{F}$ & 76.3 & $\begin{array}{r}11-09-70 \\
5-12-71\end{array}$ & $\begin{array}{l}1.0 \\
3.3\end{array}$ & $\begin{array}{l}18.3 \\
16.0\end{array}$ \\
\hline 94 & $\mathbf{s}$ & 17.0 & $\begin{array}{r}11-09-70 \\
5-12-71\end{array}$ & $\begin{array}{l}5.3 \\
5.6\end{array}$ & $\begin{array}{l}14.2 \\
13.9\end{array}$ \\
\hline 95 & $\mathbf{S}$ & 9.3 & $\begin{array}{r}1-02-70 \\
11-09-70 \\
5-12-71 \\
10-29-71 \\
5-16-72 \\
9-22-72 \\
5-11-73 \\
10-03-73\end{array}$ & $\begin{array}{l}3.9 \\
\text { dry } \\
7.3 \\
5.3 \\
8.1 \\
6.1 \\
5.6 \\
4.6\end{array}$ & $\begin{array}{r}18.0 \\
\text { dry } \\
14.6 \\
16.6 \\
13.8 \\
15.8 \\
16.3 \\
17.3\end{array}$ \\
\hline 96 & $\mathbf{S}$ & 23.4 & $\begin{array}{r}1-02-70 \\
11-09-70 \\
5-12-71 \\
10-29-71 \\
5-16-72 \\
9-22-72 \\
5-11-73 \\
10-03-73\end{array}$ & $\begin{array}{l}5.4 \\
8.6 \\
8.6 \\
5.2 \\
7.8 \\
6.1 \\
5.6 \\
4.6\end{array}$ & $\begin{array}{l}16.5 \\
13.3 \\
13.3 \\
16.7 \\
14.1 \\
15.8 \\
16.3 \\
17.3\end{array}$ \\
\hline 97 & 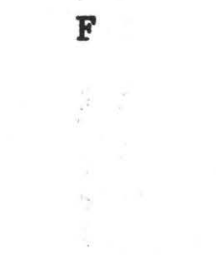 & 52.7 & $\begin{array}{r}11-09-70 \\
10-29-71 \\
5-16-72 \\
9-22-72 \\
5-11-73 \\
10-03-73\end{array}$ & $\begin{array}{l}4.1 \\
2.8 \\
4.2 \\
4.4 \\
4.8 \\
3.5\end{array}$ & $\begin{array}{l}18.1 \\
19.4 \\
18.0 \\
17.8 \\
17.4 \\
18.7\end{array}$ \\
\hline
\end{tabular}


Table 2.--Water-leve1 measurements in wells in northwest

Hillsborough County--Continued

\begin{tabular}{|c|c|c|c|c|c|}
\hline \multirow[b]{2}{*}{$\begin{array}{c}\text { Weli } \\
\text { number }\end{array}$} & \multirow[b]{2}{*}{ Aquifer $2 /$} & \multirow{2}{*}{$\begin{array}{l}\text { Depth } \\
\text { of } 3 / \\
\text { wel1- } \\
(f t)\end{array}$} & \multirow[b]{2}{*}{$\begin{array}{c}\text { Date of } \\
\text { measurement }\end{array}$} & \multicolumn{2}{|c|}{$\begin{array}{l}\text { Water leve1 } \\
\quad(\mathrm{ft})\end{array}$} \\
\hline & & & & $\begin{array}{l}\text { Above }(+) \\
\text { or below } \\
\text { land } \\
\text { surface }\end{array}$ & Altitude $4 /$ \\
\hline 99 & s & 22.0 & $\begin{array}{r}10-06-71 \\
10-29-71 \\
5-16-72 \\
9-22-72 \\
5-11-73 \\
10-03-73\end{array}$ & $\begin{array}{r}11.7 \\
6.3 \\
8.1 \\
6.6 \\
5.7 \\
5.0\end{array}$ & $\begin{array}{l}10.6 \\
16.0 \\
14.2 \\
15.7 \\
16.6 \\
17.2\end{array}$ \\
\hline 100 & $\mathrm{~S}$ & 19.3 & $\begin{array}{r}5-11-73 \\
10-03-73\end{array}$ & $\begin{array}{l}7.3 \\
6.2\end{array}$ & $\begin{array}{l}18.1 \\
19.2\end{array}$ \\
\hline 101 & S & 22.0 & $10-03-73$ & 2.4 & 20.0 \\
\hline 102 & S & 22.0 & $\begin{array}{r}10-04-71 \\
10-29-71 \\
5-16-72 \\
9-22-72\end{array}$ & $\begin{array}{r}10.0 \\
7.3 \\
8.8 \\
8.6\end{array}$ & $\begin{array}{l}14.1 \\
16.8 \\
15.3 \\
15.5\end{array}$ \\
\hline 103 & $\mathbf{S}$ & 21.0 & $\begin{array}{r}10-29-71 \\
5-16-72 \\
9-22-72 \\
5-11-73 \\
10-03-73\end{array}$ & $\begin{array}{l}5.3 \\
9.6 \\
7.5 \\
6.8 \\
6.0\end{array}$ & $\begin{array}{l}18.6 \\
14.3 \\
16.4 \\
17.1 \\
17.9\end{array}$ \\
\hline 104 & s & 14.0 & $\begin{array}{r}10-29-71 \\
5-16-72 \\
9-22-72\end{array}$ & $\begin{array}{r}6.9 \\
10.5 \\
8.9\end{array}$ & $\begin{array}{l}17.7 \\
14.1 \\
15.7\end{array}$ \\
\hline 105 & $\mathbf{s}$ & 14.5 & $\begin{array}{r}10-29-71 \\
5-16-72 \\
9-22-72 \\
5-11-73 \\
10-03-73\end{array}$ & $\begin{array}{r}9.3 \\
11.2 \\
10.8 \\
10.5 \\
9.4\end{array}$ & $\begin{array}{l}12.6 \\
10.7 \\
11.1 \\
11.4 \\
12.5\end{array}$ \\
\hline 106 & $\mathbf{F}$ & 49.4 & $\begin{array}{r}11-09-70 \\
5-12-71 \\
10-29-71 \\
5-16-72 \\
9-22-72 \\
5-11-73 \\
10-03-73\end{array}$ & $\begin{array}{l}3.6 \\
5.6 \\
2.1 \\
3.4 \\
5.0 \\
4.1 \\
2.8\end{array}$ & $\begin{array}{l}17.3 \\
15.3 \\
18.8 \\
17.5 \\
15.9 \\
16.8 \\
18.1\end{array}$ \\
\hline 107 & $\mathbf{s}$ & 23.5 & $\begin{array}{r}11-09-70 \\
5-12-71 \\
10-29-71 \\
5-16-72 \\
9-22-72 \\
5-11-73 \\
10-03-73\end{array}$ & $\begin{array}{l}14.1 \\
14.3 \\
11.6 \\
13.7 \\
13.7 \\
13.0 \\
12.0\end{array}$ & $\begin{array}{l}6.9 \\
6.7 \\
9.4 \\
7.3 \\
7.3 \\
8.0 \\
9.0\end{array}$ \\
\hline
\end{tabular}


Table 2.--Water-1evel measurements in wells in northwest

Hillsborough County--Continued

\begin{tabular}{|c|c|c|c|c|c|}
\hline \multirow[b]{2}{*}{$\begin{array}{l}\text { We11 } 1 / \\
\text { number }\end{array}$} & \multirow[b]{2}{*}{ Aquifer $2 /$} & \multirow{2}{*}{$\begin{array}{l}\text { Depth } \\
\text { of } \\
\text { we11 } \frac{3}{} / \\
(f t)\end{array}$} & \multirow[b]{2}{*}{$\begin{array}{c}\text { Date of } \\
\text { measurement }\end{array}$} & \multicolumn{2}{|c|}{$\begin{array}{l}\text { Water leve1 } \\
\quad(\mathrm{ft})\end{array}$} \\
\hline & & & & $\begin{array}{c}\text { Above (+) } \\
\text { or below } \\
\text { land } \\
\text { surface }\end{array}$ & Altitude $4 /$ \\
\hline 108 & $\mathrm{~S}$ & 14.5 & $\begin{array}{r}11-09-70 \\
5-12-71 \\
10-29-71 \\
5-16-72 \\
9-22-72 \\
5-11-73 \\
10-03-73\end{array}$ & $\begin{array}{l}13.8 \\
\text { dry } \\
11.1 \\
13.3 \\
13.5 \\
12.8 \\
11.7\end{array}$ & $\begin{array}{l}6.9 \\
\text { dry } \\
9.6 \\
7.4 \\
7.2 \\
7.9 \\
9.0\end{array}$ \\
\hline 109 & $S$ & 29.0 & $\begin{array}{r}2-13-69 \\
11-09-70 \\
5-13-71 \\
10-27-71 \\
5-24-72 \\
10-17-72\end{array}$ & $\begin{array}{l}6.8 \\
7.8 \\
8.6 \\
4.0 \\
7.6 \\
6.8\end{array}$ & $\begin{array}{l}30.6 \\
29.6 \\
28.8 \\
33.4 \\
29.8 \\
30.6\end{array}$ \\
\hline 110 & $\mathbf{S}$ & 13.0 & $\begin{array}{r}2-13-69 \\
11-09-70 \\
5-13-71 \\
10-27-71 \\
5-24-72 \\
5-18-73 \\
10-03-73\end{array}$ & $\begin{array}{l}5.2 \\
7.4 \\
8.1 \\
3.6 \\
7.3 \\
6.6 \\
3.2\end{array}$ & $\begin{array}{l}32.2 \\
30.0 \\
29.3 \\
33.8 \\
30.1 \\
30.8 \\
34.2\end{array}$ \\
\hline 113 & $\mathbf{S}$ & 22.0 & $10-03-73$ & 4.6 & 19.4 \\
\hline 114 & F & 46.2 & $10-03-73$ & 4.3 & 19.6 \\
\hline 115 & $\mathbf{S}$ & 13.5 & $\begin{array}{r}11-09-70 \\
5-12-71 \\
10-29-71 \\
5-16-72 \\
9-22-72 \\
5-11-73 \\
10-03-73\end{array}$ & $\begin{array}{r}11.1 \\
11.3 \\
7.4 \\
10.3 \\
10.6 \\
9.7 \\
8.4\end{array}$ & $\begin{array}{r}8.3 \\
8.1 \\
12.0 \\
9.1 \\
8.8 \\
9.7 \\
11.0\end{array}$ \\
\hline 116 & $\mathbf{F}$ & 43.5 & $\begin{array}{r}11-09-70 \\
5-12-71 \\
10-29-71 \\
5-16-72 \\
9-22-72 \\
5-11-73 \\
10-03-73\end{array}$ & $\begin{array}{r}2.8 \\
4.5 \\
.9 \\
2.5 \\
2.8 \\
3.0 \\
1.8\end{array}$ & $\begin{array}{l}17.0 \\
15.3 \\
18.9 \\
17.3 \\
17.0 \\
16.8 \\
18.0\end{array}$ \\
\hline
\end{tabular}


Table 2.--Water-level measurements in wells in northwest Hillsborough County--Continued

\begin{tabular}{|c|c|c|c|c|c|}
\hline \multirow[b]{2}{*}{${ }_{\text {number }}^{\text {We11 }} 1 /$} & \multirow{2}{*}{ Aquifer ${ }^{2 /}$} & \multirow{2}{*}{$\begin{array}{l}\text { Depth } \\
\text { of } \\
\text { wel1 } 3 / \\
\text { (ft) }\end{array}$} & \multirow[b]{2}{*}{$\begin{array}{c}\text { Date of } \\
\text { measurement }\end{array}$} & \multicolumn{2}{|c|}{$\begin{array}{l}\text { Water leve1 } \\
\text { (ft) }\end{array}$} \\
\hline & & & & $\begin{array}{l}\text { Above (t) } \\
\text { or below } \\
\text { land } \\
\text { surface }\end{array}$ & Altitude $4 /$ \\
\hline 117 & $\mathbf{s}$ & 9.1 & $\begin{array}{r}11-09-70 \\
5-12-71 \\
10-29-71 \\
5-16-72 \\
9-22-72 \\
5-11-73 \\
10-03-73\end{array}$ & $\begin{array}{l}9.0 \\
\text { dry } \\
2.6 \\
8.5 \\
6.7 \\
6.1 \\
4.8\end{array}$ & $\begin{array}{l}13.6 \\
\text { dry } \\
20.0 \\
14.1 \\
15.9 \\
16.5 \\
17.8\end{array}$ \\
\hline 118 & $\mathrm{~S}$ & 24.0 & $\begin{array}{r}11-09-70 \\
5-12-71 \\
10-29-71 \\
5-16-72 \\
9-22-72 \\
5-11-73 \\
10-03-73\end{array}$ & $\begin{array}{l}9.4 \\
9.6 \\
4.0 \\
8.5 \\
6.7 \\
6.1 \\
4.8\end{array}$ & $\begin{array}{l}13.2 \\
13.0 \\
18.6 \\
14.1 \\
15.9 \\
16.5 \\
17.8\end{array}$ \\
\hline 119 & $\mathbf{F}$ & 41.8 & $\begin{array}{r}11-09-70 \\
5-12-71 \\
10-29-71 \\
5-16-72 \\
9-22-72 \\
5-11-73 \\
10-03-73\end{array}$ & $\begin{array}{l}8.2 \\
8.7 \\
3.4 \\
7.7 \\
5.9 \\
6.5 \\
5.2\end{array}$ & $\begin{array}{l}13.8 \\
13.3 \\
18.6 \\
14.3 \\
16.1 \\
15.5 \\
16.8\end{array}$ \\
\hline 121 & $\mathrm{~S}$ & 29.0 & $\begin{array}{r}5-12-71 \\
10-29-71 \\
5-16-72 \\
9-22-72 \\
5-11-73\end{array}$ & $\begin{array}{r}4.6 \\
+\quad .2 \\
3.1 \\
2.1 \\
3.3\end{array}$ & $\begin{array}{l}14.0 \\
18.8 \\
15.5 \\
16.5 \\
15.3\end{array}$ \\
\hline 122 & $\mathrm{~S}$ & 10.2 & $\begin{array}{r}11-09-70 \\
5-12-71 \\
10-29-71 \\
5-16-72 \\
9-22-72 \\
5-11-73\end{array}$ & $\begin{array}{l}3.6 \\
4.5 \\
1.7 \\
3.9 \\
2.3 \\
2.9\end{array}$ & $\begin{array}{l}14.6 \\
13.7 \\
16.5 \\
14.3 \\
15.9 \\
15.3\end{array}$ \\
\hline 123 & F & 48.5 & $\begin{array}{r}11-09-70 \\
5-12-71 \\
10-29-71 \\
9-22-72 \\
5-11-73\end{array}$ & $\begin{array}{r}.3 \\
1.9 \\
+1.8 \\
.2 \\
.3\end{array}$ & $\begin{array}{l}18.6 \\
16.4 \\
20.1 \\
18.1 \\
18.0\end{array}$ \\
\hline 124 & $\mathbf{S}$ & 20.2 & $\begin{array}{r}11-10-70 \\
5-13-71 \\
10-27-71\end{array}$ & $\begin{array}{r}10.5 \\
11.2 \\
6.0\end{array}$ & $\begin{array}{l}29.7 \\
29.0 \\
34.2\end{array}$ \\
\hline
\end{tabular}


Table 2.--Water-level measurements in wells in northwest Hillsborough County--Continued

\begin{tabular}{|c|c|c|c|c|c|}
\hline \multirow[b]{2}{*}{$\begin{array}{l}\text { We11 } 1 / \\
\text { number }\end{array}$} & \multirow[b]{2}{*}{ Aquifer $2 /$} & \multirow{2}{*}{$\begin{array}{l}\text { Depth } \\
\text { of } \\
\text { wel1 } 1-3 / \\
(f t)\end{array}$} & \multirow{2}{*}{$\begin{array}{c}\text { Date of } \\
\text { measurement }\end{array}$} & \multicolumn{2}{|c|}{$\begin{array}{l}\text { Water level } \\
(\mathrm{ft})\end{array}$} \\
\hline & & & & $\begin{array}{l}\text { Above (+) } \\
\text { or below } \\
\text { land } \\
\text { surface }\end{array}$ & Altitude $4 /$ \\
\hline 125 & $\mathbf{S}$ & 11.8 & $11-09-70$ & 7.6 & 32 \\
\hline 126 & $\mathrm{~S}$ & 24.0 & $11-09-70$ & 8.6 & 31 \\
\hline 127 & $\mathrm{~S}$ & 19.6 & $\begin{array}{r}10-28-71 \\
5-16-72 \\
9-22-72 \\
5-11-73 \\
10-03-73\end{array}$ & $\begin{array}{l}2.3 \\
5.1 \\
7.2 \\
7.1 \\
5.4\end{array}$ & $\begin{array}{l}23.3 \\
20.5 \\
18.4 \\
18.5 \\
20.2\end{array}$ \\
\hline 128 & $\mathrm{~F}$ & 62.5 & $\begin{array}{r}5-11-73 \\
10-03-73\end{array}$ & $\begin{array}{l}7.2 \\
5.4\end{array}$ & $\begin{array}{l}18.4 \\
20.2\end{array}$ \\
\hline 131 & $\mathrm{~S}$ & 14.0 & $\begin{array}{r}10-29-71 \\
5-16-72 \\
9-22-72 \\
5-11-73 \\
10-03-73\end{array}$ & $\begin{array}{r}7.6 \\
11.0 \\
10.0 \\
8.9 \\
7.5\end{array}$ & $\begin{array}{l}17.1 \\
13.7 \\
14.7 \\
15.8 \\
17.2\end{array}$ \\
\hline 132 & $\mathrm{~S}$ & 18.0 & $\begin{array}{r}5-13-71 \\
10-28-71 \\
5-16-72 \\
9-22-72 \\
5-11-73 \\
10-03-73\end{array}$ & $\begin{array}{l}7.4 \\
2.1 \\
4.6 \\
5.9 \\
5.6 \\
3.4\end{array}$ & $\begin{array}{l}19.9 \\
25.2 \\
22.7 \\
21.4 \\
21.7 \\
23.9\end{array}$ \\
\hline 133 & F & 110.0 & $\begin{array}{r}5-11-73 \\
10-03-73\end{array}$ & $\begin{array}{l}5.8 \\
3.6\end{array}$ & $\begin{array}{l}21.9 \\
24.1\end{array}$ \\
\hline 134 & $\mathrm{~S}$ & 12.4 & $\begin{array}{r}1-23-70 \\
11-09-70 \\
5-12-71 \\
10-29-71 \\
5-16-72 \\
9-22-72 \\
5-11-73 \\
10-03-73\end{array}$ & $\begin{array}{r}9.3 \\
11.3 \\
11.6 \\
9.8 \\
11.2 \\
11.3 \\
10.9 \\
9.7\end{array}$ & $\begin{array}{l}8.3 \\
6.3 \\
6.0 \\
7.8 \\
6.4 \\
6.3 \\
6.7 \\
7.9\end{array}$ \\
\hline 135 & $S$ & 21.9 & $\begin{array}{r}1-23-70 \\
11-09-70 \\
5-12-71 \\
10-29-71 \\
5-16-72 \\
9-22-72 \\
5-11-73 \\
10-03-73\end{array}$ & $\begin{array}{r}9.9 \\
9.4 \\
9.7 \\
4.3 \\
5.3 \\
11.6 \\
11.3 \\
10.2\end{array}$ & $\begin{array}{r}7.9 \\
8.4 \\
8.1 \\
13.5 \\
12.5 \\
6.2 \\
6.5 \\
7.6\end{array}$ \\
\hline
\end{tabular}


Table 2.--Water-level measurements in wells in northwest Hillsborough County--Continued

\begin{tabular}{|c|c|c|c|c|c|}
\hline \multirow[b]{2}{*}{$\begin{array}{l}\text { Wel1 } 1 / \\
\text { number }\end{array}$} & \multirow[b]{2}{*}{ Aquifer $2 /$} & \multirow{2}{*}{$\begin{array}{l}\text { Depth } \\
\text { of } \\
\text { wel1 } 1 \text { / } \\
\text { (ft) }\end{array}$} & \multirow[b]{2}{*}{$\begin{array}{c}\text { Date of } \\
\text { measurement }\end{array}$} & \multicolumn{2}{|c|}{$\begin{array}{l}\text { Water leve1 } \\
(\mathrm{ft})\end{array}$} \\
\hline & & & & $\begin{array}{c}\text { Above (+) } \\
\text { or below } \\
\text { land } \\
\text { surface }\end{array}$ & Altitude $4 /$ \\
\hline 136 & F & 42.9 & $\begin{array}{r}1-23-70 \\
11-09-70 \\
10-29-71 \\
5-16-72 \\
9-22-72 \\
5-11-73 \\
10-03-73\end{array}$ & $\begin{array}{r}1.3 \\
+.5 \\
+.9 \\
.5 \\
.7 \\
1.0 \\
+\quad .2\end{array}$ & $\begin{array}{l}16.3 \\
17.1 \\
18.5 \\
17.1 \\
16.9 \\
16.6 \\
17.8\end{array}$ \\
\hline 137 & $\mathrm{~F}$ & 49.0 & $\begin{array}{r}11-09-70 \\
5-12-71 \\
10-29-71 \\
5-16-72\end{array}$ & $\begin{array}{l}5.2 \\
7.4 \\
4.6 \\
5.3\end{array}$ & $\begin{array}{l}18.0 \\
15.8 \\
18.6 \\
17.9\end{array}$ \\
\hline 138 & $\mathrm{~S}$ & 23.8 & $\begin{array}{r}11-09-70 \\
5-12-71 \\
10-29-71 \\
5-16-72 \\
5-11-73 \\
10-03-73\end{array}$ & $\begin{array}{r}9.6 \\
11.3 \\
5.3 \\
9.7 \\
6.9 \\
6.4\end{array}$ & $\begin{array}{l}13.6 \\
11.9 \\
17.9 \\
13.5 \\
16.3 \\
16.8\end{array}$ \\
\hline 139 & $S$ & 9.2 & $\begin{array}{r}11-09-70 \\
5-12-71 \\
10-29-71 \\
5-16-72 \\
9-22-72\end{array}$ & $\begin{array}{l}\text { dry } \\
\text { dry } \\
5.3 \\
\text { dry } \\
9.3\end{array}$ & $\begin{array}{r}\text { dry } \\
\text { dry } \\
17.8 \\
\text { dry } \\
13.8\end{array}$ \\
\hline 140 & $\mathrm{~S}$ & 14.0 & $10-29-71$ & 8.2 & 17.0 \\
\hline 141 & $\mathrm{~S}$ & 14.0 & $10-29-71$ & 9.3 & 15.7 \\
\hline 142 & $\mathrm{~S}$ & 13.4 & $\begin{array}{r}11-09-70 \\
5-12-71 \\
10-29-71 \\
5-16-72 \\
9-22-72 \\
5-11-73 \\
10-03-73\end{array}$ & $\begin{array}{r}12.0 \\
\text { dry } \\
9.3 \\
11.7 \\
11.9 \\
11.0 \\
9.7\end{array}$ & $\begin{array}{r}7.3 \\
\text { dry } \\
10.0 \\
7.6 \\
7.4 \\
8.3 \\
9.6\end{array}$ \\
\hline 143 & $\mathrm{~S}$ & 20.8 & $\begin{array}{r}11-09-70 \\
5-12-71 \\
10-29-71 \\
5-16-72 \\
9-22-72 \\
5-11-73 \\
10-03-73\end{array}$ & $\begin{array}{r}12.0 \\
12.5 \\
9.5 \\
11.7 \\
11.9 \\
11.1 \\
9.8\end{array}$ & $\begin{array}{l}7.4 \\
6.9 \\
9.9 \\
7.7 \\
7.5 \\
8.3 \\
9.6\end{array}$ \\
\hline
\end{tabular}


Table 2.--Water-1eve1 measurements in wells in northwest Hillsborough County--Continued

\begin{tabular}{|c|c|c|c|c|c|}
\hline \multirow[b]{2}{*}{$\begin{array}{l}\text { Well } 1 / \\
\text { number }\end{array}$} & \multirow[b]{2}{*}{ Aquifer $2 /$} & \multirow{2}{*}{$\begin{array}{l}\text { Depth } \\
\text { of } \\
\text { we11 } 3 / \\
\text { (ft) }\end{array}$} & \multirow[b]{2}{*}{$\begin{array}{c}\text { Date of } \\
\text { measurement }\end{array}$} & \multicolumn{2}{|c|}{$\begin{array}{l}\text { Water level } \\
\quad(f t)\end{array}$} \\
\hline & & & & $\begin{array}{l}\text { Above ( }+ \text { ) } \\
\text { or below } \\
\text { land } \\
\text { surface }\end{array}$ & Altitude 4/ $^{4 /}$ \\
\hline 144 & $\mathrm{~F}$ & 49.7 & $\begin{array}{r}1-23-70 \\
11-09-70 \\
5-12-71 \\
10-29-71 \\
5-16-72 \\
9-22-72 \\
5-11-73 \\
10-03-73\end{array}$ & $\begin{array}{l}1.3 \\
2.8 \\
4.8 \\
1.5 \\
2.9 \\
3.1 \\
3.4 \\
2.1\end{array}$ & $\begin{array}{l}18.2 \\
16.7 \\
14.7 \\
18.0 \\
16.6 \\
16.4 \\
16.1 \\
17.4\end{array}$ \\
\hline 145 & $\mathrm{~S}$ & 17.5 & $\begin{array}{l}11-09-70 \\
10-17-72 \\
10-03-73\end{array}$ & $\begin{array}{l}7.2 \\
6.3 \\
4.0\end{array}$ & $\begin{array}{l}29.5 \\
30.4 \\
32.7\end{array}$ \\
\hline 146 & $S$ & 33.3 & $\begin{array}{r}11-09-70 \\
10-17-72 \\
5-11-73 \\
10-03-73\end{array}$ & $\begin{array}{l}8.1 \\
7.4 \\
7.6 \\
4.9\end{array}$ & $\begin{array}{l}28.7 \\
29.4 \\
29.2 \\
31.9\end{array}$ \\
\hline 147 & $\mathrm{~F}$ & 45.8 & $\begin{array}{r}10-17-72 \\
5-11-73 \\
10-03-73\end{array}$ & $\begin{array}{r}10.5 \\
10.7 \\
7.9\end{array}$ & $\begin{array}{l}25.3 \\
25.1 \\
27.9\end{array}$ \\
\hline 148 & S & 13.7 & $\begin{array}{r}2-11-69 \\
11-09-70\end{array}$ & $\begin{array}{l}3.5 \\
5.3\end{array}$ & $\begin{array}{l}33.4 \\
31.6\end{array}$ \\
\hline 149 & $\mathrm{~S}$ & 26.5 & $\begin{array}{r}2-11-69 \\
11-09-70\end{array}$ & $\begin{array}{l}5.7 \\
8.1\end{array}$ & $\begin{array}{l}33.3 \\
30.9\end{array}$ \\
\hline 150 & S & 15.0 & $10-29-71$ & 9.8 & 16.3 \\
\hline 151 & $\mathrm{~S}$ & 14.0 & $\begin{array}{r}10-29-71 \\
5-16-72\end{array}$ & $\begin{array}{r}9.3 \\
12.9\end{array}$ & $\begin{array}{l}15.5 \\
11.9\end{array}$ \\
\hline 152 & $\mathrm{~F}$ & 38.6 & $\begin{array}{r}11-09-70 \\
5-12-71 \\
10-29-71 \\
5-16-72 \\
9-22-72 \\
5-11-73 \\
10-03-73\end{array}$ & $\begin{array}{r}7.9 \\
10.0 \\
6.2 \\
7.9 \\
7.8 \\
8.4 \\
6.9\end{array}$ & $\begin{array}{l}16.6 \\
14.5 \\
18.3 \\
16.6 \\
16.7 \\
16.1 \\
17.6\end{array}$ \\
\hline 153 & S & 14.4 & $\begin{array}{r}11-09-70 \\
5-12-71 \\
10-29-71 \\
5-16-72 \\
9-22-72 \\
5-11-73 \\
10-03-73\end{array}$ & $\begin{array}{r}11.1 \\
13.2 \\
7.6 \\
11.1 \\
10.0 \\
11.2 \\
7.6\end{array}$ & $\begin{array}{l}12.7 \\
10.6 \\
16.2 \\
12.7 \\
13.8 \\
12.6 \\
16.2\end{array}$ \\
\hline
\end{tabular}


Table 2.--Water-1eve1 measurements in we11s in northwest Hillsborough County--Continued

\begin{tabular}{|c|c|c|c|c|c|}
\hline \multirow[b]{2}{*}{$\mathrm{Well}_{\text {number }} 1 /$} & \multirow[b]{2}{*}{ Aquifer $2 /$} & \multirow{2}{*}{$\begin{array}{l}\text { Depth } \\
\text { of } \\
\text { wel1 } 3 / \\
\text { (ft) }\end{array}$} & \multirow[b]{2}{*}{$\begin{array}{c}\text { Date of } \\
\text { measurement }\end{array}$} & \multicolumn{2}{|c|}{$\begin{array}{l}\text { Water level } \\
\text { ( } f t)\end{array}$} \\
\hline & & & & $\begin{array}{l}\text { Above (+) } \\
\text { or below } \\
\text { land } \\
\text { surface }\end{array}$ & Altitude $4 /$ \\
\hline 154 & $\mathrm{~S}$ & 15.0 & $\begin{array}{r}11-09-70 \\
5-12-71 \\
10-29-71 \\
5-16-72 \\
9-22-72 \\
5-11-73\end{array}$ & $\begin{array}{l}13.0 \\
13.6 \\
10.3 \\
\text { dry } \\
13.2 \\
12.1\end{array}$ & $\begin{array}{l}6.5 \\
5.9 \\
9.2 \\
\text { dry } \\
6.3 \\
7.4\end{array}$ \\
\hline 155 & $\mathbf{F}$ & 43.9 & $\begin{array}{r}11-09-70 \\
5-12-71 \\
10-29-71 \\
5-16-72 \\
9-22-72 \\
5-11-73 \\
10-03-73\end{array}$ & $\begin{array}{r}2.1 \\
4.1 \\
.7 \\
2.2 \\
2.3 \\
4.0 \\
2.8\end{array}$ & $\begin{array}{l}17.4 \\
15.4 \\
18.8 \\
17.3 \\
17.2 \\
15.5 \\
16.7\end{array}$ \\
\hline 156 & $\mathbf{F}$ & 79.0 & $\begin{array}{r}10-28-71 \\
5-16-72\end{array}$ & $\begin{array}{l}4.4 \\
6.6\end{array}$ & $\begin{array}{l}21 \\
18\end{array}$ \\
\hline 157 & $\mathrm{~s}$ & 17.0 & $\begin{array}{r}10-29-71 \\
5-16-72 \\
9-22-72 \\
5-11-73 \\
10-03-73\end{array}$ & $\begin{array}{r}8.4 \\
12.9 \\
11.4 \\
11.5 \\
8.6\end{array}$ & $\begin{array}{l}15.3 \\
10.8 \\
12.3 \\
12.2 \\
15.1\end{array}$ \\
\hline 158 & S & 13.9 & $\begin{array}{r}11-09-70 \\
5-12-71 \\
10-29-71 \\
5-16-72 \\
9-22-72 \\
5-11-73 \\
10-03-73\end{array}$ & $\begin{array}{r}8.4 \\
\text { dry } \\
8.4 \\
13.0 \\
11.7 \\
11.6 \\
8.3\end{array}$ & $\begin{array}{r}14.8 \\
\text { dry } \\
14.8 \\
10.2 \\
11.5 \\
11.6 \\
14.9\end{array}$ \\
\hline 159 & F & 39.6 & $\begin{array}{r}5-12-71 \\
10-29-71 \\
5-16-72 \\
9-22-72 \\
5-11-73\end{array}$ & $\begin{array}{r}10.1 \\
5.7 \\
8.3 \\
8.3 \\
8.4\end{array}$ & $\begin{array}{l}13.3 \\
17.7 \\
15.1 \\
15.1 \\
15.0\end{array}$ \\
\hline 160 & F & 80.0 & $5-16-72$ & 4.9 & 20 \\
\hline 161 & S & 18.9 & $\begin{array}{r}10-28-71 \\
5-16-72 \\
9-22-72 \\
5-11-73 \\
10-03-73\end{array}$ & $\begin{array}{r}4.7 \\
10.5 \\
10.5 \\
10.4 \\
8.7\end{array}$ & $\begin{array}{l}16.3 \\
10.5 \\
10.5 \\
10.6 \\
12.3\end{array}$ \\
\hline
\end{tabular}


Table 2.--Water-1eve1 measurements in wells in northwest Hillsborough County--Continued

\begin{tabular}{|c|c|c|c|c|c|}
\hline \multirow[b]{2}{*}{$\begin{array}{c}\text { We11 } 1 / \\
\text { number }\end{array}$} & \multirow[b]{2}{*}{ Aquifer $2 /$} & \multirow{2}{*}{$\begin{array}{l}\text { Depth } \\
\text { of } \\
\text { well } 3 / \\
(f t)\end{array}$} & \multirow[b]{2}{*}{$\begin{array}{c}\text { Date of } \\
\text { measurement }\end{array}$} & \multicolumn{2}{|c|}{$\begin{array}{l}\text { Water leve1 } \\
(\mathrm{ft})\end{array}$} \\
\hline & & & & $\begin{array}{l}\text { Above ( }(+) \\
\text { or below } \\
\text { land } \\
\text { surface }\end{array}$ & Altitude $e^{4 /}$ \\
\hline 162 & $\mathbf{s}$ & 18.0 & $\begin{array}{r}5-13-71 \\
10-28-71 \\
5-16-72 \\
9-22-72 \\
5-11-73 \\
10-03-73\end{array}$ & $\begin{array}{r}\text { dry } \\
14.2 \\
16.8 \\
17.2 \\
16.4 \\
14.7\end{array}$ & $\begin{array}{r}\text { dry } \\
12.7 \\
10.1 \\
9.7 \\
10.5 \\
12.2\end{array}$ \\
\hline 163 & $\mathbf{F}$ & 55.0 & $\begin{array}{r}5-13-71 \\
10-28-71 \\
5-16-72 \\
10-17-72 \\
5-11-73 \\
10-03-73\end{array}$ & $\begin{array}{l}7.7 \\
2.5 \\
4.7 \\
5.7 \\
5.4 \\
3.6\end{array}$ & $\begin{array}{l}17.2 \\
22.4 \\
20.2 \\
19.2 \\
19.5 \\
21.3\end{array}$ \\
\hline 164 & $\mathbf{F}$ & 1,120 & $\begin{array}{r}11-10-70 \\
5-13-71 \\
10-28-71 \\
5-24-72 \\
10-16-72 \\
5-17-73 \\
10-04-73\end{array}$ & $\begin{array}{r}2.8 \\
7.0 \\
.8 \\
4.6 \\
4.3 \\
3.7 \\
2.4\end{array}$ & $\begin{array}{l}21.2 \\
17.0 \\
23.2 \\
19.4 \\
19.7 \\
20.3 \\
21.6\end{array}$ \\
\hline 165 & $\mathbf{F}$ & -- & $\begin{array}{r}11-10-70 \\
5-13-71 \\
10-28-71 \\
10-16-72 \\
5-17-73\end{array}$ & $\begin{array}{l}4.2 \\
7.4 \\
1.0 \\
6.0 \\
4.4\end{array}$ & $\begin{array}{l}21 \\
18 \\
24 \\
19 \\
21\end{array}$ \\
\hline 166 & $\mathbf{F}$ & 90 & $\begin{array}{r}11-09-70 \\
5-13-71 \\
10-27-71 \\
5-24-72 \\
10-16-72 \\
5-18-73 \\
10-04-73\end{array}$ & $\begin{array}{r}3.5 \\
8.0 \\
.6 \\
5.5 \\
5.1 \\
3.9 \\
2.3\end{array}$ & $\begin{array}{l}24 \\
19 \\
26 \\
22 \\
22 \\
23 \\
25\end{array}$ \\
\hline 167 & $\mathbf{F}$ & 60.0 & $\begin{array}{r}11-10-70 \\
5-13-71 \\
10-27-71 \\
5-24-72 \\
10-16-72 \\
5-18-73 \\
10-04-73\end{array}$ & $\begin{array}{l}6.1 \\
7.1 \\
3.1 \\
6.2 \\
5.6 \\
5.2 \\
3.4\end{array}$ & $\begin{array}{l}29 \\
28 \\
32 \\
29 \\
29 \\
30 \\
31\end{array}$ \\
\hline
\end{tabular}


Table 2.--Water-1evel measurements in we11s in northwest Hillsborough County--Continued

\begin{tabular}{|c|c|c|c|c|c|}
\hline \multirow[b]{2}{*}{$\begin{array}{l}\text { We11 } 1 / \\
\text { number }\end{array}$} & \multirow[b]{2}{*}{ Aquifer $2 /$} & \multirow{2}{*}{$\begin{array}{l}\text { Depth } \\
\text { of } \\
\text { wel1 } 3 / \\
(f t)\end{array}$} & \multirow[b]{2}{*}{$\begin{array}{c}\text { Date of } \\
\text { measurement }\end{array}$} & \multicolumn{2}{|c|}{$\begin{array}{l}\text { Water leve1 } \\
(\mathrm{ft})\end{array}$} \\
\hline & & & & $\begin{array}{l}\text { Above }(+) \\
\text { or below } \\
\text { land } \\
\text { surface }\end{array}$ & Altitude $4 /$ \\
\hline 168 & F & 556 & $\begin{array}{r}11-13-70 \\
10-14-71 \\
9-06-72 \\
5-16-73\end{array}$ & $\begin{array}{l}24.0 \\
22.2 \\
23.8 \\
27.3\end{array}$ & $\begin{array}{l}34.6 \\
36.4 \\
34.8 \\
31.3\end{array}$ \\
\hline 169 & F & 194.0 & $\begin{array}{r}11-10-70 \\
5-13-71 \\
10-27-71 \\
5-24-72 \\
9-28-72 \\
5-17-73\end{array}$ & $\begin{array}{l}15.6 \\
22.9 \\
13.9 \\
20.5 \\
20.4 \\
16.7\end{array}$ & $\begin{array}{l}24 \\
17 \\
26 \\
20 \\
20 \\
23\end{array}$ \\
\hline 170 & $\mathbf{F}$ & -- & $\begin{array}{r}11-10-70 \\
5-13-71 \\
10-27-71 \\
5-24-72 \\
10-16-72 \\
5-17-73 \\
10-04-73\end{array}$ & $\begin{array}{l}14.9 \\
20.9 \\
10.6 \\
17.9 \\
17.7 \\
13.2 \\
13.0\end{array}$ & $\begin{array}{l}22 \\
16 \\
26 \\
19 \\
19 \\
24 \\
24\end{array}$ \\
\hline 171 & $\mathbf{F}$ & 1,200 & $\begin{array}{r}9-22-72 \\
5-17-73 \\
10-04-73\end{array}$ & $\begin{array}{r}11.0 \\
9.8 \\
7.5\end{array}$ & $\begin{array}{l}18.0 \\
19.2 \\
21.5\end{array}$ \\
\hline 174 & $\mathbf{F}$ & 280 & $\begin{array}{r}11-10-70 \\
5-13-71 \\
5-24-72 \\
10-16-72\end{array}$ & $\begin{array}{r}8.6 \\
14.2 \\
11.9 \\
11.0\end{array}$ & $\begin{array}{l}16 \\
11 \\
13 \\
14\end{array}$ \\
\hline
\end{tabular}

1/ Location of wells shown in figures 2 and 3.

2/ F, Floridan aquifer; $S$, surficial aquifer.

3/ Depths shown to nearest tenth of a foot are measured; other depths are reported.

4/ Altitudes shown to nearest tenth of a foot above National Geodetic Vertical Datum of 1929, determined by level instrument; other altitudes estimated from topographic maps. 
Table 3.--Lithologic logs of wells in northwest Hillsborough County

Lithologic description

Thickness

Depth

(ft)

(ft)

We11 56

Sand, brownish-black, fine-grained, with some peat -------

Sand, 1ight-gray, fine-grained --

Sand, very light-gray, very fine-grained, sugary --_-_----

2.0

2.0

3.5

5.5

Sand, brownish-gray, very fine-grained, with some silt

and clay

8.5

14.0

Clay, bluish-gray, plastic, sandy

11.5

25.5

Clay, light olive-gray, plastic, with some sand and

limestone fragments

5.0

30.5

Clay, medium-gray, plastic, with some limestone

fragments

10.0

40.5

4.5

45.0

We11 57

Sand, pinkish-gray, very fine-grained, sugary _-_-_-_-_ 5.0

5.0

Sand, dusky-brown, very fine-grained, with some silt -..--

15.5

20.5

Clay, greenish-yellow, plastic, with some sand and

limestone fragments

5.0

25.5

Clay, greenish-gray, plastic

5.0

30.5

Sand, light brownish-gray, fine-grained, with some clay --

8.0

38.5

Limestone, white, soft and porous, fossiliferoūs

1.0

39.5

We11 57A

Sand, dusky-brown, fine-grained

Sand, moderate-brown, very fine-grained, silty

1.5

1.5

Sand, pale yellowish-brown, fine-grained, sugary

.5

2.0

2.0

4.0

Sand, as above, but pale orange

10.0

14.0

Sand, pale-brown, fine-grained, silty and clayey

1.0

15.0

Clay, light olive-gray, friable, sandy and silty

7.0

22.0

Clay, dusky yellowish-green, plastic, with some sand --.--

3.0

25.0

Clay, greenish-black, plastic

1.0

26.0

Clay, greenish-gray, plastic, with some limestone

fragments

3.0

29.0

Clay, yellowish-gray, plastic, with some limestone

fragments

Limestone, white, soft, clayey

3.0

32.0

Limestone, greenish-gray, soft, clayey

8.0

40.0

2.0

42.0

Chert

1.0

43.0 
Table 3.--Lithologic logs of we11s in northwest Hillsborough County--Continued

\begin{tabular}{|c|c|c|}
\hline Lithologic description & $\begin{array}{c}\text { Thickness } \\
\text { (ft) }\end{array}$ & $\begin{array}{r}\text { Depth } \\
\text { (ft) }\end{array}$ \\
\hline
\end{tabular}

We11 59

Sand, dark-brown, fine- to medium-grained --_-

3.0

3.0

Sand, light-brown, fine- to medium-grained -

Sand, light-tan, fine-grained, silty --

Clay, bluish-gray, plastic, with some sand --

Sand, white, very fine-grained, sugary -

Sand, light-tan, plastic, silty

1.0

4. 0

1.0

5.0

$1.0 \quad 6.0$

$1.5 \quad 7.5$

1.59 .0

We11 60

Sand, black, medium- to fine-grained

1.5

1.5

Sand, reddish-brown, fine-grained -

1.5

3.0

Sand, white, fine- to very fine-grained, with some silt --

1.0

4.0

Sand, tan to light-gray, very fine-grained, silty ---_---

3.0

7.0

Sand, purplish-brown, very fine-grained, clayey

3.0

10.0

We11 61

Sand, dark-brown, fine- to medium-grained, with some clay

Sand, yellow to reddish-brown, fine- to medium-grained ---

Sand, gray to white, medium- to very fine-grained, sugary

Clay and sand, bluish clay, plastic, with very finegrained sand

We11 62

Sand, gray, fine- to medium-grained

2.0

2.0

Sand, dark-brown, fine-grained

3.0

5.0

Sand, light-tan, very fine-grained, sugary

7.0

12.0

Sand and silt, light-brown, very fine-grained

1.0

13.0

Sand, dark-brown, fine-grained, clayey

3.0

16.0

Sand, white, fine-grained

3.0

19.0

Sand, light-brown, fine-grained, clayey

8.0

27.0

Sand, light-brown, fine-grained, silty

1.0

28.0

Clay, bluish-gray, plastic, with some sand

10.0

38.0

Clay, bluish-green, plastic, with some fine-grained sand -

9.0

47.0 
Table 3.--Lithologic logs of wells in northwest Hillsborough County--Continued

\section{Lithologic description}

Thickness

Depth

(ft)

(ft)

We11 63

Sand, gray, fine- to medium-grained

1.0

1.0

Sand, white, fine- to medium-grained

2.0

3.0

Sand, reddish-brown, fine- to medium-grained

2.0

5.0

Sand and silt, fine- to medium-grained, light gray

2.0

7.0

Sand, light-gray, fine-grained, with some silt

2.0

9.0

We11 65

Sand

Sand, dark-brown, fine-grained

3.0

3.0

4.0

7.0

Sand, as above, but light-brown to white

5.0

12.0

Sand, brown, silty and clayey

8.0

20.0

Clay, sandy

2.0

22.0

We11 67

Sand, light-tan, very fine-grained, with much peat

Sand, orange-gray, fine- to medium-grained with some silt

Clay, light olive-gray, friable, sandy, and silty --_------

Limestone, white, very dense, hard --

9.0

19.5

Clay, light olive-brown, plastic, with some sand --------

4.0

23.5

Limestone, yellowish-gray, very dense, hard

10.5

34.0

11.0

45.0

\section{We11 76}

Sand, dark-gray, fine-grained, with some organic material

Sand, pale yellowish-brown, fine-grained, with lense of black sand

Sand, pale-brown, very fine-grained, silty

Sand, pale yellowish-brown, fine-grained, silty -..-_-_-_-

Sand, light olive-gray, very fine-grained, silty, clayey - 
Table 3.--Lithologic logs of wells in northwest Hillsborough County--Continued

\begin{tabular}{ll} 
Lithologic description & $\begin{array}{c}\text { Thickness } \\
\text { (ft) }\end{array}$ \\
\hline
\end{tabular}

Wel1 85

Sand, very pale yellowish-brown, very fine-grained -------

Sand, pale-brown, very fine-grained, silty --------

Clay, olive-gray, with some fine-grained sand ----------

Limestone, yellowish-gray, dense

5.5

5.5

25.0

30.5

11.5

42.0

8.5

50.5

We11 86

Sand, dark-gray, fine- to medium-grained, quartz, with some peat --

Sand, pale yellowish-brown, fine- to medium-grained ------

Sand, pale-brown, very fine-grained, silty --

Sand, very pale-orange, fine- to medium-grained ----------

Sand, pale-brown, very fine-grained, silty

2.0

2.0

7.0

9.0

10.0

19.0

5.0

24.0

1.0

25.0

We11 89

Sand, dark-gray, very fine-grained, silty ---------------

Sand, dark yellowish-orange, very fine-grained, silty ----

2.5

2.5

Sand, very pale orange, very fine-grained, with thin lense of clay -

Sand, very pale orange, very fine-grained, with some silt

Sand, very pale orange, very fine-grained, silty, and clayey

3.0

5.5

$5.0 \quad 10.5$

$5.0 \quad 15.5$

5.0

20.5

Clay, greenish-gray, plastic, with some sand

5.0

25.5

\section{We11 93}

Sand, gray, fine- to very fine-grained -

Sand, brownish-black, very fine-grained, with some silt --

Sand, light-gray, very fine-grained, with some silt -- -

Sand, brownish-black, very fine-grained, silty

Clay, brownish-black, plastic, with some sand and peat ---

Sand, olive-black, fine- to very fine-grained with some silt

Sand, yellowish-brown, very fine-grained, silty and

20.0

59.0

$$
\text { clayey }
$$

6.5

65.5

Clay, greenish-gray, plastic, with some sand 
Table 3.--Lithologic logs of wells in northwest Hillsborough County--Continued

Lithologic description $\quad \begin{gathered}\text { Thickness } \\ (\mathrm{ft})\end{gathered} \quad \begin{gathered}\text { Depth } \\ (\mathrm{ft})\end{gathered}$

We11 97

Sand, dark-gray, fine- to medium-grained --_-_-_-_-_-_--

2.0

2.0

Sand, blackish-gray, fine- to medium-grained -------------

2.0

4.0

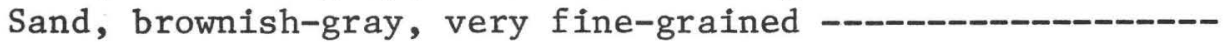

10.0

14.0

Sand, pale-yellow, very fine-grained, with some silt -----

20.0

34.0

Clay, olive-gray, plastic, sandy --_-_-_---_-_-_-----

11.5

45.5

Limestone, greenish-gray, hard and dense _-_-_-_-_._-_ 10.0

55.5

We11 106

Sand, very light-gray, very fine-grained --_-_-_-_-_----

Sand, 1ight-gray, very fine-grained -_-_-_-_-_-_-_-_-_-_

2.0

2.0

Sand, pale yellowish-brown, very fine-grained with some

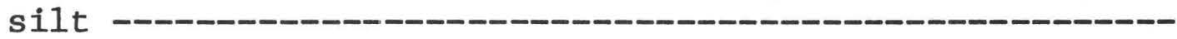

3.5

5.5

Sand, brownish-gray, very fine-grained, silty -_-_-_-_-_-

Sand, pinkish-gray, fine-grained -_-_-_-_-_-_-_-_-_-_-_-_-

Sand, pale yellowish-brown, very fine-grained, silty -----

Clay, olive-gray, plastic, with some sand -_-_.-_-_-_-_-

Clay, pale yellowish-green, friable, sticky --_-_-_-_-_---

5.0

10.5

10.0

20.5

5.0

25.5

$10.0 \quad 35.5$

Limestone, yellowish-gray, dense, with some sand and clay

5.0

40.5

5.0

45.5

5.0

50.5

We11 109

Sand, dusky-brown, fine-grained, quartz, with some peat -3.0

Clay, grayish-green, plastic, with some fine-grained sand

Clay, light-gray, plastic, sandy --_-_-_-_-_-_-_-_-_---

5.0

8.0

Sand, 1ight-gray, fine-grained, clayey --_-_-_-_-_-_-_---

2.0

10.0

Sand, pinkish-gray, fine-grained, sugary -------

5.5

15.5

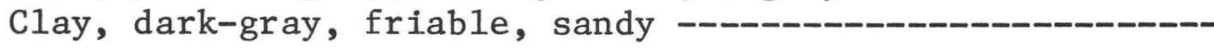

5.0

20.5

Clay, yellowish-green, very stiff, with some fine-grained sand

5.0

25.5

5.0

30.5

We11 112

Sand, dusky-brown, fine-grained, quartz, with some silt

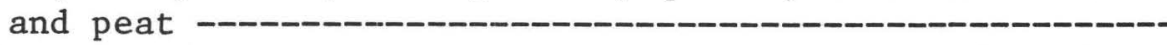

Clay, light-gray, plastic, with some fine-grained sand ---

Clay, yellowish-green, plastic, with some fine-grained

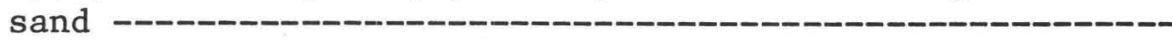

Clay and limestone, white, with some shell fragments ----- 
Table 3.--Lithologic logs of wells in northwest Hillsborough County--Continued

\begin{tabular}{|c|c|}
\hline Lithologic description & $\begin{array}{l}\text { Thickness } \\
\text { (ft) }\end{array}$ \\
\hline
\end{tabular}

We11 116

Sand, very pale-orange, fine- to medium-grained

5.5

5.5

Sand, pale-brown, fine-grained, silty

20.0

25.5

Clay, light olive-gray, plastic, sandy

5.0

30.5

Clay, light olive-gray, plastic, very sandy -_._-_._-_.-.

8.5

39.0

Limestone, yellowish-gray, clayey

6.5

45.5

We11 119

Sand, light brownish-gray, fine- to medium-grained -----Sand, yellowish-brown, very fine-grained, with some sand -

Sand, as above, but dark yellowish-brown --

3.5

5.5

Sand, as above, but pale yellowish-brown --

5.0

10.5

Sand, brownish-gray, fine- to very fine-grained, with some

29.5

40.0

silt and clay

5.5

45.5

Clay, olive-gray, plastic, with some fine-grained sand ---

Clay, very light-gray, with much limestone

10.0

55.5

3.5

59.0

We11 123

Sand, dark-gray, fine- to medium-grained with some peat --

Sand, brown
material

3.5

5.5

Sand, dark yellowish-brown, very fine-grained, with some silt

13.5

19.0

Sand, very pale-orange, fine-grained

11.5

30.5

Clay, dark greenish-gray, plastic, with some sand

5.0

35.5

Clay and limestone, dark greenish-gray, plastic, limestone, soft and chalky 
Table 3.--Lithologic logs of wells in northwest Hillsborough County--Continued

\begin{tabular}{|c|c|}
\hline Lithologic description & $\begin{array}{l}\text { Thickness } \\
\text { (ft) }\end{array}$ \\
\hline
\end{tabular}

We11 124

Sand, tanish-gray, medium-grained

3.0

3.0

Sand, light-tan, clayey, with reddish-brown layers --_-_--

Clay, red, plastic, sandy -

1.0

4.0

Clay, bluish-gray, plastic -

1.5

5.5

Clay, grayish-green, plastic, sandy -

1.0

6.5

Clay, purplish-brown, plastic, sandy

2.5

9.0

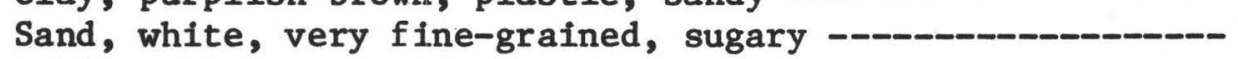

7.0

16.0

Sand, yellow, very fine-grained, sugary --_-_-_-_-_-_--

Sand, light tanish-purple, clayey, with shell fragments --

Clay, bluish-green, plastic, with shell fragments --------

Clay, blue, plastic, with many fossils

4.0

20.0

4.0

24.0

3.0

27.0

12.0

39.0

3.0

42.0

Well 126

Sand, dusky-brown, very fine-grained, quartz _-_-_-_-_ Clay, 1ight-gray, plastic, with some sand --_-_-_-_-_-_-_Sand, white, fine-grained, sugary

5.0

5.0

Clay and limestone, white, with some sand and shell

fragments

10.0

15.0

5.0

20.0

10.5

30.5

Wel1 133A

Sand, pale yellowish-brown, fine- to very fine-grained ---

10.5

10.5

Sand, as above, but silty --

10.0

20.5

Sand, dark yellowish-brown, very fine-grained, very silty

10.0

30.5

Clay, yellowish-brown, plastic, sandy and silty ----------

5.0

35.5

Sand, pale yellowish-brown, fine-grained, with some silt -

5.0

40.5

Clay, yellowish-brown, friable, sandy and silty --..---.-

5.0

45.5

Clay, olive-black, plastic, with some sand --- ---------

Clay, olive-gray, with fragments of limestone -----------

5.0

50.5

Limestone, gray, dense and hard

5.0

55.5

10.0

65.5 
Table 3.--Lithologic logs of wells in northwest Hillsborough County--Continued

Lithologic description

We11 136
Sand, grayish-orange, medium-grained

Sand, pale-brown, fine-grained, silty

Sand, pale yellowish-brown, medium- to fine-grained, silty

Clay, olive-gray, plastic, sandy

Limestone, light olive-gray, with sand and chert
Thickness

(ft)

Depth

(ft)

\section{Well 137}

Sand, dark-gray, fine- to medium-grained

10.5

10.5

15.0

25.5

5.0

30.5

13.0

43.5

1.0

44.5

Sand, brown, fine-grained, with some silt

2.0

2.0

Sand, pale yellowish-brown, very fine-grained

13.5

17.5

Clay, olive-gray, plastic, sandy

12.5

5.0

15.5

33.0

45.5

Limestone, yellowish-gray, with some clay and sand

50.5

\section{We11 144}

Sand, orange-gray, medium-grained

Sand, very pale-orange, fine- to medium-grained

10.5

10.5

Sand, pale yellowish-brown, fine- to medium-grained, silty

Clay, olive-gray, plastic, sandy

Clay, olive-gray, plastic, with some sand -

Limestone, very pale-orange, clayey

15.0

30.5

10.0

40.5

5.0

45.5

5.0

50.5

\section{We11 147}

Sand, dark-brown, fine-grained, with some silt, clay, and peat -

Sand, as above, with solid waste material from a landfil1 -

Clay, light-gray, plastic, sandy --

Clay, 1ight-tan, friable, sandy

$5.0 \quad 5.0$

Sand, white, fine-grained, sugary

Cavity

Limestone, 11ght-gray, with some clay and shel1 fragments.

$\begin{array}{rr}5.0 & 10.0 \\ 10.0 & 20.0 \\ 10.0 & 30.0 \\ 9.0 & 39.0 \\ 7.0 & 46.0 \\ 4.5 & 50.5\end{array}$


Table 3.--Lithologic logs of wells in northwest Hillsborough County--Continued

Lithologic description

We11 149
Thickness

(ft)

Depth

(ft)
Sand, gray, fine-grained

Clay, dark yellowish-brown, plastic, with some fine-

grained sand - white, very fine-grained, with limonite streaks ---

Sand, light-gray, fine-grained, sugary --

Sand, gray, fine-grained, clayey, with limestone --------

Clay and limestone, 1ight-gray, with some sand

$$
5.0
$$

\section{5}

15.0

5.0

4.5

5.5
5.0

10.5

25.5

30.5

35.0

40.5

\section{We11 152}

Sand, 1ight-gray, fine-grained

Sand, pinkish-gray, fine-grained -

Sand, brownish-gray, fine-grained, silty --_-

Sand, pinkish-gray, fine-grained --_--_---_---------

Sand, yellowish-brown, fine-grained, with silt and clay -Clay, olive-gray, plastic, with some fine-grained sand --Limestone, yellowish-gray, clayey
2.0

3.5

5.0

8.5

6.5

8.5

6.5
2.0

5.5

10.5

19.0

25.5

34.0

40.5

\section{Wel1 154}

Sand, yellowish-gray, very fine-grained -Sand, white, very fine-grained --_-_Sand, pinkish-gray, fine-grained -----------------Sand, pinkish-gray, fine-grained, sugary, appears to be water-bearing

Sand, yellowish-gray, very fine-grained

Sand, white, very fine-grained

Sand, pinkish-gray, very fine-grained

Clay, light olive-gray, plastic, with some fine-grained sand

Clay and limestone

Clay, greenish-gray, plastic, with some sand

Limestone, very pale-orange, dense, with some sand and
2.0

5.0

2.0

23.0

7.0

30.0

$2.0 \quad 32.0$

$4.0 \quad 36.0$

$4.0 \quad 40.0$

$4.0 \quad 44.0$ 
Table 3.--Lithologic logs of wells in northwest Hillsborough County--Continued

\section{Lithologic description}

Thickness

Depth

(ft)

(ft)

We11 159

Sand, brownish-black, fine-grained

Sand, dark yellowish-orange, fine-grained

2.0

2.0

Sand, pale-brown, fine-grained, silty

3.5

5.5

Clay, olive-gray, plastic, with some sand

23.5

29.0

Limestone, yellowish-gray, clayey, with some sand

5.0

34.0

41.0

\section{Wel1 161}

Sand, yellowish-gray, medium-grained

4.0

4.0

Sand, white, medium- to fine-grained

3.0

7.0

Sand, pale yellowish-brown, fine-grained, with some silt -

33.0

40.0

Clay, dark-brown, plastic, with some sand

4.0

44.0

Clay, dark yell
grained sand

3.0

47.0 
Table 4.--Surface-water sites at the Rocky Creek landfil1

\begin{tabular}{|c|c|c|}
\hline $\begin{array}{l}\text { Site } \\
\text { number }\end{array}$ & $\begin{array}{c}\text { Identification } \\
\text { number }\end{array}$ & Site name \\
\hline $\mathrm{SW}-1$ & 2802230823430.00 & Rocky Creek at railroad bridge \\
\hline SW-2 & 2802270823430.02 & Sump pit \\
\hline $\mathrm{SW}-3$ & 2802270823431.00 & West perimeter ditch \\
\hline SW-4 & 2802360823439.02 & Rocky Creek near we11 92 \\
\hline SW-5 & 2802350823425.00 & Oxidation pond \\
\hline SW-6 & 2802270823417.00 & South perimeter ditch \\
\hline SW-7 & 2802470823431.00 & West perimeter ditch \\
\hline $\mathrm{SW}-8$ & 2802470823417.00 & North perimeter ditch \\
\hline SW-9 & 2802510823358.00 & Rocky Creek at Wilsky Road \\
\hline
\end{tabular}




\begin{tabular}{|c|c|c|c|c|c|c|c|c|c|c|c|c|}
\hline 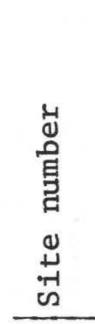 & 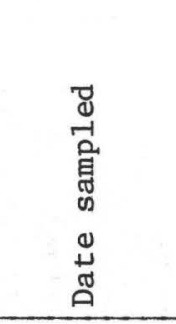 & 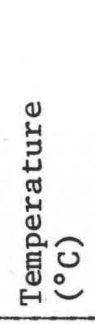 & 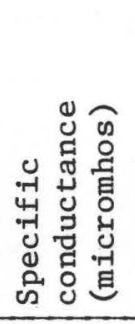 & 票 & 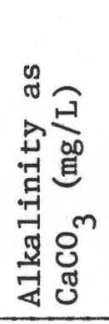 & 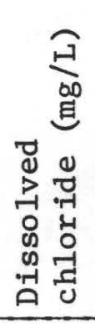 & 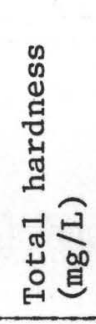 & 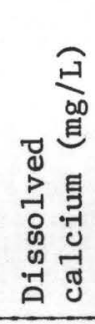 & 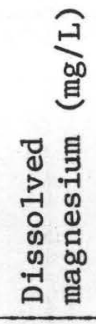 & 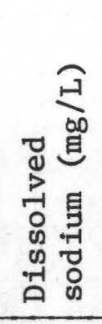 & 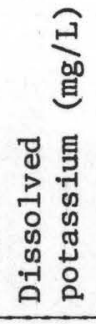 & 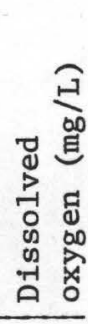 \\
\hline SW-1 & $\begin{array}{r}8-05-70 \\
9-18-70 \\
10-27-70 \\
12-03-70 \\
1-14-71\end{array}$ & $\begin{array}{l}29.0 \\
29.5 \\
22.0 \\
17.5 \\
20.0\end{array}$ & $\begin{array}{l}169 \\
106 \\
180 \\
199 \\
220\end{array}$ & $\begin{array}{l}6.7 \\
6.1 \\
6.3 \\
6.4 \\
7.0\end{array}$ & $\begin{array}{l}69 \\
18 \\
68 \\
78 \\
60\end{array}$ & $\begin{array}{l}-- \\
12 \\
13 \\
14 \\
19\end{array}$ & $\begin{array}{l}-- \\
27 \\
71 \\
74 \\
78\end{array}$ & $\begin{array}{l}-- \\
-- \\
-- \\
-- \\
--\end{array}$ & $\begin{array}{l}-- \\
-- \\
-- \\
--\end{array}$ & $\begin{array}{l}-- \\
-- \\
-- \\
-- \\
--\end{array}$ & $\begin{array}{l}-- \\
-- \\
-- \\
--\end{array}$ & $\begin{array}{l}4.1 \\
2.6 \\
-- \\
6.3 \\
6.2\end{array}$ \\
\hline & $\begin{array}{l}2-24-71 \\
3-30-71 \\
4-28-71 \\
6-15-71 \\
7-27-71\end{array}$ & $\begin{array}{l}19.0 \\
16.5 \\
25.0 \\
25.5 \\
28.0\end{array}$ & $\begin{array}{l}198 \\
180 \\
192 \\
208 \\
195\end{array}$ & $\begin{array}{l}6.5 \\
6.5 \\
-- \\
6.4 \\
6.4\end{array}$ & $\begin{array}{l}54 \\
62 \\
-- \\
64 \\
56\end{array}$ & $\begin{array}{l}18 \\
13 \\
14 \\
14 \\
16\end{array}$ & $\begin{array}{l}66 \\
65 \\
76 \\
71 \\
61\end{array}$ & $\begin{array}{l}-- \\
-- \\
-- \\
--\end{array}$ & $\begin{array}{l}-- \\
-- \\
-- \\
--\end{array}$ & $\begin{array}{l}-- \\
-- \\
-- \\
--\end{array}$ & $\begin{array}{l}-- \\
-- \\
-- \\
--\end{array}$ & $\begin{array}{l}-- \\
3.6 \\
6.1 \\
2 \\
1.7\end{array}$ \\
\hline & $\begin{array}{r}9-22-71 \\
10-29-71 \\
11-22-71 \\
12-27-71 \\
1-26-72\end{array}$ & $\begin{array}{l}27.0 \\
22.0 \\
-- \\
-- \\
23.0\end{array}$ & $\begin{array}{r}91 \\
110 \\
129 \\
147 \\
153\end{array}$ & $\begin{array}{l}6.0 \\
6.0 \\
-- \\
6.3 \\
--\end{array}$ & $\begin{array}{l}12 \\
20 \\
-- \\
44 \\
--\end{array}$ & $\begin{array}{l}10 \\
14 \\
13 \\
13 \\
12\end{array}$ & $\begin{array}{l}32 \\
34 \\
36 \\
46 \\
61\end{array}$ & $\begin{array}{l}-- \\
-- \\
12 \\
-- \\
--\end{array}$ & $\begin{array}{l}-- \\
-- \\
1.5 \\
--\end{array}$ & $\begin{array}{l}-- \\
-- \\
6.5 \\
-- \\
--\end{array}$ & $\begin{array}{l}-- \\
-- \\
1.3 \\
--\end{array}$ & $\begin{array}{l}-- \\
-- \\
-- \\
-- \\
3.2\end{array}$ \\
\hline & $\begin{array}{r}3-17-72 \\
7-18-72 \\
10-25-72 \\
4-26-73 \\
8-10-73\end{array}$ & $\begin{array}{l}-- \\
-- \\
-- \\
-- \\
--\end{array}$ & $\begin{array}{l}130 \\
203 \\
214 \\
160 \\
210\end{array}$ & $\begin{array}{l}-- \\
-- \\
-- \\
-- \\
--\end{array}$ & $\begin{array}{l}-- \\
-- \\
-- \\
-- \\
--\end{array}$ & $\begin{array}{l}14 \\
21 \\
16 \\
13 \\
20\end{array}$ & $\begin{array}{r}43 \\
130 \\
89 \\
53 \\
64\end{array}$ & $\begin{array}{r}9 \\
32 \\
30 \\
17 \\
20\end{array}$ & $\begin{array}{c}4.9 \\
13 \\
3.4 \\
2.5 \\
3.4\end{array}$ & $\begin{array}{l}1.5 \\
-- \\
9.8 \\
9.6 \\
12\end{array}$ & $\begin{array}{l}2.1 \\
3.1 \\
-- \\
2 \\
4\end{array}$ & $\begin{array}{l}-- \\
-- \\
-- \\
--\end{array}$ \\
\hline & $\begin{array}{l}10-15-73 \\
12-20-73\end{array}$ & -- & $\begin{array}{l}129 \\
185\end{array}$ & -- & - & $\begin{array}{l}17 \\
22\end{array}$ & $\begin{array}{l}40 \\
69\end{array}$ & $\begin{array}{l}12 \\
21\end{array}$ & $\begin{array}{l}2.4 \\
3.9\end{array}$ & $12^{8.5}$ & $\begin{array}{l}3.3 \\
7.8\end{array}$ & -- \\
\hline SW-2 & $\begin{array}{r}8-04-70 \\
10-27-70 \\
12-03-70 \\
1-14-71 \\
2-24-71\end{array}$ & $\begin{array}{l}-- \\
22.5 \\
18.5 \\
23.0 \\
22.5\end{array}$ & $\begin{array}{r}95 \\
240 \\
220 \\
254 \\
174\end{array}$ & $\begin{array}{l}6.4 \\
6.4 \\
6.3 \\
6.9 \\
6.2\end{array}$ & $\begin{array}{r}35 \\
95 \\
86 \\
116 \\
56\end{array}$ & $\begin{array}{r}8 \\
16 \\
15 \\
18 \\
18\end{array}$ & $\begin{array}{r}14 \\
101 \\
46 \\
88 \\
50\end{array}$ & $\begin{array}{l}-- \\
-- \\
-- \\
--\end{array}$ & $\begin{array}{l}-- \\
-- \\
--\end{array}$ & $\begin{array}{l}-- \\
-- \\
-- \\
--\end{array}$ & $\begin{array}{l}-- \\
-- \\
-- \\
--\end{array}$ & $\begin{array}{l}4.5 \\
-- \\
3.4 \\
5.6 \\
--\end{array}$ \\
\hline & $\begin{array}{l}3-30-71 \\
4-28-71 \\
6-15-71 \\
7-28-71 \\
9-22-71\end{array}$ & $\begin{array}{l}22.0 \\
31.0 \\
28.0 \\
29.0 \\
34.0\end{array}$ & $\begin{array}{l}270 \\
322 \\
307 \\
460 \\
188\end{array}$ & $\begin{array}{l}6.9 \\
-- \\
6.7 \\
7.1 \\
6.5\end{array}$ & $\begin{array}{r}104 \\
\overline{96} \\
240 \\
102\end{array}$ & $\begin{array}{l}22 \\
28 \\
24 \\
25 \\
17\end{array}$ & $\begin{array}{r}98 \\
114 \\
101 \\
186 \\
68\end{array}$ & $\begin{array}{l}-- \\
-- \\
-- \\
--\end{array}$ & $\begin{array}{l}-- \\
-- \\
-- \\
--\end{array}$ & $\begin{array}{l}-- \\
-- \\
-- \\
--\end{array}$ & $\begin{array}{l}-- \\
-- \\
-- \\
--\end{array}$ & $\begin{array}{l}3.4 \\
4.2 \\
1 \\
1.4 \\
--\end{array}$ \\
\hline & $\begin{array}{l}10-28-71 \\
11-22-71\end{array}$ & $\begin{array}{l}21.0 \\
17.5\end{array}$ & $\begin{array}{l}185 \\
220\end{array}$ & 6.0 & $\begin{array}{l}48 \\
--\end{array}$ & $\begin{array}{l}22 \\
21\end{array}$ & $\begin{array}{l}61 \\
76\end{array}$ & $\overline{23}$ & -- & $\overline{12}$ & $\overline{1.6}$ & -- \\
\hline & $12-27-71$ & -- & 228 & 6.5 & 820 & 24 & 76 & - & -- & - & - & - \\
\hline & $1-26-72$ & 19.0 & 303 & - & -- & 31 & 140 & -- & -- & -- & -- & -- \\
\hline & $3-17-72$ & -- & 220 & - & -- & 26 & 64 & 17 & 5 & 8 & 15 & -- \\
\hline & $7-18-72$ & -- & 450 & -- & -- & 35 & 250 & 43 & 34 & -- & 7.7 & -- \\
\hline & $\begin{array}{r}10-25-72 \\
4-27-73\end{array}$ & -- & $\begin{array}{l}540 \\
420\end{array}$ & - & -- & $\begin{array}{l}51 \\
36\end{array}$ & $\begin{array}{l}180 \\
130\end{array}$ & $\begin{array}{l}62 \\
46\end{array}$ & $\begin{array}{l}6 \\
4.4\end{array}$ & $\begin{array}{l}36 \\
18\end{array}$ & $\begin{array}{l}9.4 \\
5.8\end{array}$ & -- \\
\hline
\end{tabular}




\begin{tabular}{|c|c|c|c|c|c|c|c|c|c|c|c|c|}
\hline 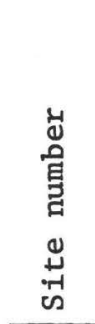 & 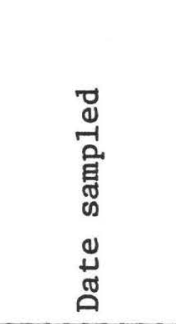 & 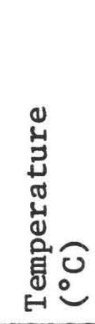 & 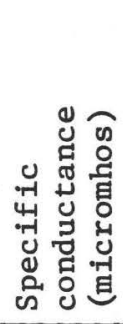 & 営 & 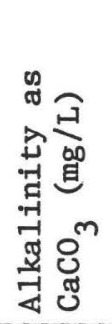 & 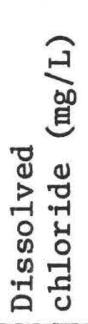 & 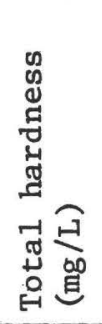 & 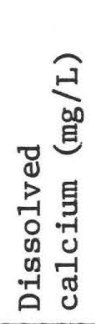 & 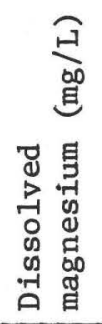 & 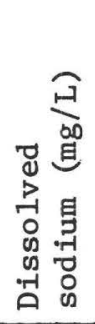 & 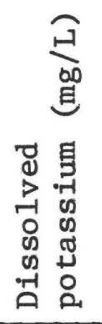 & 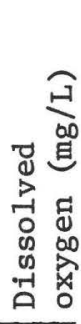 \\
\hline \multirow[t]{17}{*}{ SW-3 } & $3-31-70$ & 31.0 & 51 & -- & -- & 9 & -- & -- & -- & -- & -- & -- \\
\hline & $4-20-70$ & 33.0 & 150 & -- & -- & 12 & 54 & -- & -- & -- & -- & -- \\
\hline & $6-01-70$ & 34.0 & 115 & -- & -- & 15 & -- & -- & -- & -- & -- & -- \\
\hline & $6-02-70$ & 24.5 & 162 & -- & -- & 18 & -- & -- & -- & -- & -- & -- \\
\hline & $6-15-70$ & 34.5 & 340 & -- & -- & -- & -- & -- & -- & -- & -- & 8 \\
\hline & $6-16-70$ & 30.0 & -- & 6.7 & -- & -- & -- & -- & -- & -- & -- & -- \\
\hline & $6-25-70$ & 31.5 & 45 & 6.3 & 11 & 8 & 13 & -- & -- & -- & -- & -- \\
\hline & $7-14-70$ & 36.0 & 80 & -- & 0 & 9 & 26 & -- & -- & -- & -- & -- \\
\hline & $8-04-70$ & 30.0 & 103 & 6.4 & 28 & 11 & 17 & -- & -- & -- & -- & 6.4 \\
\hline & $1-14-71$ & 28.0 & 252 & 5.8 & 80 & 23 & 90 & -- & -- & -- & -- & -- \\
\hline & $2-24-71$ & 24.0 & 182 & 5.9 & 52 & 17 & 62 & -- & -- & -- & -- & -- \\
\hline & $3-30-71$ & 23.0 & 288 & 7.1 & 90 & 27 & 94 & -- & -- & -- & -- & 7 \\
\hline & $6-15-71$ & 27.0 & 224 & 6.5 & 80 & 22 & 71 & -- & -- & -- & -- & 1.6 \\
\hline & $7-28-71$ & 29.5 & 376 & 7.3 & 196 & 24 & 169 & -- & -- & -- & -- & 1.9 \\
\hline & $9-22-71$ & 25.0 & 85 & 6.0 & 20 & 10 & 34 & -- & -- & -- & -- & -- \\
\hline & $10-28-71$ & 24.0 & 83 & 4.3 & -- & 14 & 20 & -- & -- & -- & -- & -- \\
\hline & $1-26-72$ & 19.0 & 300 & -- & -- & 32 & 110 & -- & -- & -- & -- & 4.6 \\
\hline \multirow[t]{17}{*}{ SW-4 } & $8-05-70$ & 30.0 & 174 & 7.2 & 69 & 15 & 42 & -- & -- & -- & -- & 7.4 \\
\hline & $9-18-70$ & 30.0 & 106 & 6.7 & -- & 14 & 25 & -- & -- & -- & -- & 7.6 \\
\hline & $10-27-70$ & 21.5 & 178 & 6.5 & 67 & 5 & 67 & -- & -- & -- & -- & -- \\
\hline & $12-02-70$ & 17.0 & 199 & 6.5 & 86 & 24 & 74 & -- & -- & -- & -- & 4.3 \\
\hline & $1-14-71$ & 19.0 & 222 & 6.7 & 66 & 19 & 79 & -- & -- & -- & -- & 3.8 \\
\hline & $2-24-71$ & 18.0 & 195 & 6.5 & 54 & 19 & 61 & -- & -- & -- & -- & -- \\
\hline & $3-31-71$ & 15.5 & -- & 6.5 & 64 & -- & -- & - & -- & -- & -- & 2.5 \\
\hline & $4-28-71$ & -- & 174 & -- & -- & 15 & 60 & -- & -- & -- & -- & 4.6 \\
\hline & $6-14-71$ & 26.0 & 185 & 6.5 & 56 & 14 & 65 & -- & -- & -- & -- & 2 \\
\hline & $7-28-71$ & 26.0 & 174 & 6.3 & 60 & 15 & 62 & -- & -- & -- & -- & 1 \\
\hline & $9-21-71$ & 27.0 & 85 & 7.0 & 16 & 10 & 28 & -- & -- & -- & -- & -- \\
\hline & $10-28-71$ & 22.0 & 105 & 6.0 & 14 & 14 & 35 & -- & -- & -- & -- & -- \\
\hline & $1-26-72$ & -- & 152 & -- & -- & 14 & 72 & -- & -- & -- & -- & -- \\
\hline & $3-17-72$ & -- & -- & -- & -- & -- & 42 & 12 & 3.1 & 2 & 1 & -- \\
\hline & $7-18-72$ & -- & 190 & -- & -- & 19 & 100 & -- & 4.6 & -- & 2.5 & -- \\
\hline & $10-25-72$ & -- & 204 & -- & -- & 17 & 71 & 23 & 3.3 & 9.6 & 3 & -- \\
\hline & $4-24-73$ & -- & 390 & -- & -- & 13 & 180 & 66 & 4.8 & 9 & .9 & -- \\
\hline \multirow[t]{5}{*}{ SW-5 } & $4-23-70$ & 29.0 & 76 & -- & -- & 10 & -- & -- & -- & -- & -- & -- \\
\hline & $6-01-70$ & 31.0 & -- & -- & -- & -- & -- & -- & -- & -- & -- & -- \\
\hline & $6-16-70$ & 30.0 & 150 & 6.8 & -- & -- & -- & -- & -- & -- & -- & 4 \\
\hline & $6-25-70$ & 34.0 & 121 & 6.3 & 18 & 18 & 18 & -- & -- & -- & -- & -- \\
\hline & $7-14-70$ & 38.0 & 107 & -- & -- & 12 & 22 & -- & -- & -- & -- & -- \\
\hline
\end{tabular}




\begin{tabular}{|c|c|c|c|c|c|c|c|c|c|c|c|c|}
\hline 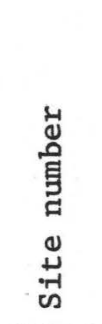 & 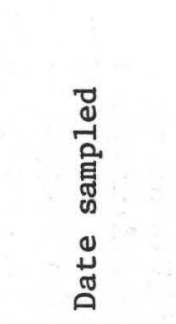 & 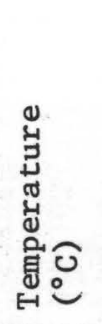 & 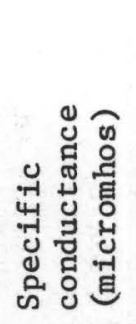 & 爱 & 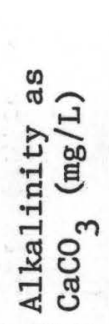 & 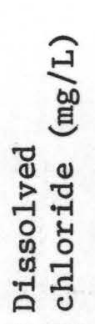 & 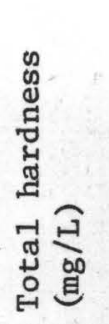 & 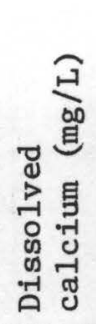 & 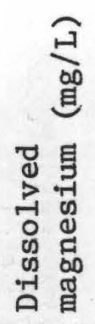 & 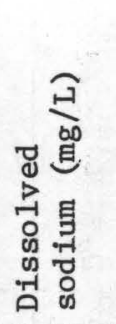 & 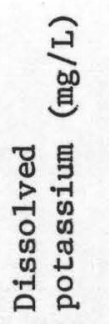 & 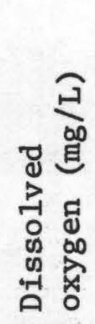 \\
\hline \multirow[t]{13}{*}{ SW-5 } & $8-04-70$ & 34.0 & 85 & 6.2 & 48 & 13 & 6 & -- & -- & - & - & 5.6 \\
\hline & $10-26-70$ & 28.5 & 77 & 7.9 & 10 & 10 & 19 & -- & -- & -- & -- & -- \\
\hline & $7-27-71$ & 36.0 & 97 & 5.5 & 4 & 12 & 21 & -- & -- & -- & -- & 4.2 \\
\hline & $9-22-71$ & 29.5 & 228 & 7.3 & 84 & 17 & 64 & -- & -- & -- & -- & -- \\
\hline & $10-28-71$ & 31.0 & 760 & -- & -- & 56 & 220 & -- & -- & -- & -- & -- \\
\hline & $11-22-71$ & -- & 625 & -- & -- & 45 & 180 & 58 & 8.6 & 43 & 28 & -- \\
\hline & $12-28-71$ & -- & 262 & 6.4 & 70 & 24 & 64 & -- & -- & -- & -- & - \\
\hline & $1-26-72$ & 28.0 & 87 & -- & -- & 10 & 40 & -- & -- & -- & - & 8.7 \\
\hline & $3-15-72$ & -- & 1,100 & -- & -- & 62 & 370 & 120 & 19 & 60 & 44 & - \\
\hline & $7-18-72$ & -- & 354 & -- & -- & 24 & 230 & 74 & 12 & 19 & 1.8 & -- \\
\hline & $4-23-73$ & -- & 130 & -- & -- & 70 & 44 & 14 & 2.1 & 8 & .2 & -- \\
\hline & $10-16-73$ & -- & 73 & -- & -- & 11 & 15 & 5 & .8 & 5 & 3.3 & -- \\
\hline & $12-19-73$ & -- & 126 & -- & -- & 20 & 47 & 16 & 1.7 & 8.9 & 19 & -- \\
\hline \multirow[t]{20}{*}{ SW-6 } & $3-31-70$ & 27.0 & 54 & -- & -- & 9 & -- & -- & -- & -- & - & -- \\
\hline & $4-20-70$ & 31.0 & -- & -- & -- & -- & -- & -- & -- & -- & -- & -- \\
\hline & $4-23-70$ & 31.0 & 41 & -- & -- & 8 & 9 & -- & -- & -- & -- & -- \\
\hline & $6-01-70$ & 33.0 & 46 & -- & -- & 12 & -- & -- & -- & -- & -- & -- \\
\hline & $6-16-70$ & 29.0 & 50 & 5.4 & -- & - & -- & -- & -- & -- & -- & 6.7 \\
\hline & $6-25-70$ & 33.0 & 40 & 6.2 & 6 & 7 & 10 & -- & -- & -- & -- & -- \\
\hline & $7-14-70$ & 37.0 & 47 & -- & -- & 7 & 10 & -- & -- & -- & -- & -- \\
\hline & $8-05-70$ & 22.0 & 57 & 5.4 & 8 & 9 & 17 & -- & -- & -- & -- & 4.4 \\
\hline & $9-18-70$ & 32.0 & 67 & 5.6 & 7 & 10 & 13 & -- & -- & -- & -- & 8.4 \\
\hline & $10-26-70$ & 29.5 & 54 & 6.0 & 10 & 8 & 14 & -- & -- & -- & -- & -- \\
\hline & $1-13-71$ & 19.5 & 55 & 5.2 & 6 & 8 & 16 & -- & -- & -- & -- & 8.1 \\
\hline & $2-26-71$ & 19.5 & 54 & 5.3 & 6 & 9 & 14 & -- & -- & -- & -- & -- \\
\hline & $3-31-71$ & 21.5 & 54 & 5.5 & 8 & 8 & 14 & -- & -- & -- & -- & 8.9 \\
\hline & $4-28-71$ & 25.0 & 73 & -- & -- & 13 & 29 & -- & -- & -- & -- & 7.5 \\
\hline & $7-28-71$ & 27.0 & 51 & 5.4 & 8 & 8 & 21 & -- & -- & -- & -- & 4.2 \\
\hline & $9-22-71$ & 33.0 & 75 & 6.3 & 12 & 8 & 24 & -- & -- & -- & -- & -- \\
\hline & $10-29-71$ & 24.0 & 57 & 5.1 & 4 & 11 & -- & -- & - & -- & -- & -- \\
\hline & $1-26-72$ & 23.0 & 58 & -- & -- & 8 & 28 & -- & -- & -- & -- & 7.5 \\
\hline & $3-16-72$ & -- & 60 & -- & -- & 9 & 12 & 0 & 2.8 & 0 & 0 & -- \\
\hline & $4-27-73$ & -- & 166 & -- & -- & 25 & 38 & 12 & 2 & 14 & 1.5 & -- \\
\hline \multirow[t]{8}{*}{ SW-7 } & $2-05-70$ & 8.0 & 37 & 6.0 & 4 & 8 & 8 & -- & -- & -- & -- & -- \\
\hline & $3-31-70$ & 27.0 & 54 & -- & -- & 12 & -- & -- & - & -- & -- & -- \\
\hline & $4-23-70$ & 35.0 & 38 & -- & -- & 8 & 8 & -- & -- & -- & -- & -- \\
\hline & $6-01-70$ & 34.5 & 48 & -- & -- & 17 & -- & -- & -- & -- & -- & -- \\
\hline & $7-14-70$ & 39.5 & 136 & -- & -- & 7 & 14 & -- & -- & -- & -- & -- \\
\hline & $1-14-71$ & 7.2 & 252 & -- & -- & 23 & 90 & -- & -- & -- & -- & -- \\
\hline & $9-22-71$ & 33.0 & 67 & 7.3 & 22 & 6 & 26 & -- & -- & -- & -- & -- \\
\hline & $10-28-71$ & 24.0 & 52 & 5.2 & 32 & 12 & 12 & -- & - & -- & -- & -- \\
\hline
\end{tabular}




\begin{tabular}{|c|c|c|c|c|c|c|c|c|c|c|c|c|}
\hline 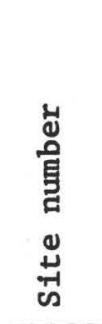 & 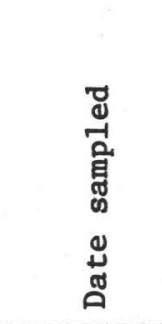 & 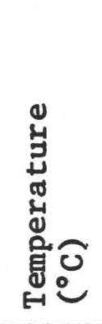 & 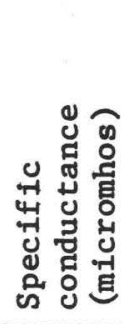 & 罟 & 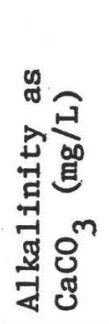 & 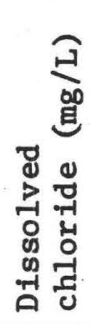 & 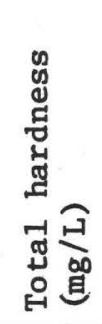 & 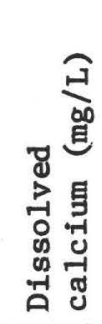 & 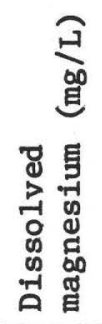 & 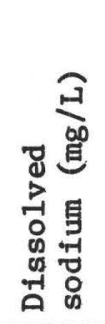 & 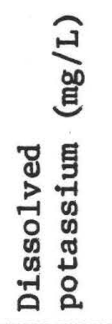 & 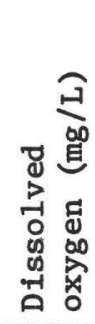 \\
\hline SW-8 & $\begin{array}{l}3-31-70 \\
4-23-70 \\
6-01-70 \\
6-16-70 \\
6-25-70\end{array}$ & $\begin{array}{l}27.0 \\
36.0 \\
36.0 \\
32.0 \\
35.0\end{array}$ & $\begin{array}{l}54 \\
35 \\
47 \\
50 \\
33\end{array}$ & $\begin{array}{l}-- \\
-- \\
-- \\
5.4 \\
5.4\end{array}$ & $\begin{array}{l}-- \\
-- \\
-- \\
2\end{array}$ & $\begin{array}{r}10 \\
8 \\
11 \\
--\end{array}$ & $\begin{array}{l}-- \\
10 \\
-- \\
-- \\
6\end{array}$ & $\begin{array}{l}-- \\
-- \\
-- \\
-- \\
--\end{array}$ & $\begin{array}{l}-- \\
-- \\
-- \\
-- \\
5.4\end{array}$ & $\begin{array}{l}-- \\
-- \\
-- \\
-- \\
--\end{array}$ & $\begin{array}{l}-- \\
-- \\
-- \\
-- \\
--\end{array}$ & $\begin{array}{l}-- \\
-- \\
-- \\
7 \\
--\end{array}$ \\
\hline & $\begin{array}{r}7-14-70 \\
1-13-71 \\
3-31-71 \\
9-22-71 \\
10-28-71\end{array}$ & $\begin{array}{l}39.0 \\
19.5 \\
-- \\
33.0 \\
24.0\end{array}$ & $\begin{array}{l}44 \\
-- \\
54 \\
60 \\
52\end{array}$ & $\begin{array}{l}-- \\
-- \\
-- \\
5.9 \\
5.0\end{array}$ & $\begin{array}{l}-- \\
-- \\
-- \\
8 \\
5\end{array}$ & $\begin{array}{r}7 \\
15 \\
8 \\
8 \\
11\end{array}$ & $\begin{array}{r}8 \\
-- \\
14 \\
20 \\
12\end{array}$ & $\begin{array}{l}-- \\
-- \\
-- \\
-- \\
--\end{array}$ & $\begin{array}{l}-- \\
-- \\
-- \\
--\end{array}$ & $\begin{array}{l}-- \\
-- \\
-- \\
-- \\
--\end{array}$ & $\begin{array}{l}-- \\
-- \\
-- \\
-- \\
--\end{array}$ & $\begin{array}{l}-- \\
-- \\
-- \\
-- \\
--\end{array}$ \\
\hline & $\begin{array}{r}11-23-71 \\
3-17-72 \\
7-19-72\end{array}$ & $\begin{array}{l}-- \\
-- \\
--\end{array}$ & $\begin{array}{r}790 \\
55 \\
62\end{array}$ & $\begin{array}{l}-- \\
-- \\
--\end{array}$ & $\begin{array}{l}-- \\
-- \\
--\end{array}$ & $\begin{array}{r}34 \\
7 \\
12\end{array}$ & $\begin{array}{r}380 \\
15 \\
290\end{array}$ & $\begin{array}{r}120 \\
3 \\
18\end{array}$ & $\begin{array}{l}20 \\
1.7 \\
59\end{array}$ & $\begin{array}{r}19 \\
0 \\
--\end{array}$ & $\begin{array}{l}10 \\
.1 \\
1.3\end{array}$ & $\begin{array}{l}-- \\
-- \\
--\end{array}$ \\
\hline SW-9 & $\begin{array}{r}10-23-70 \\
12-03-70 \\
1-14-71 \\
2-26-71 \\
3-31-71\end{array}$ & $\begin{array}{l}23.0 \\
18.0 \\
19.0 \\
21.0 \\
15.0\end{array}$ & $\begin{array}{l}165 \\
178 \\
212 \\
156 \\
167\end{array}$ & $\begin{array}{l}6.8 \\
6.5 \\
6.8 \\
6.4 \\
6.5\end{array}$ & $\begin{array}{l}52 \\
58 \\
52 \\
32 \\
48\end{array}$ & $\begin{array}{l}15 \\
16 \\
21 \\
16 \\
12\end{array}$ & $\begin{array}{l}51 \\
58 \\
67 \\
48 \\
63\end{array}$ & $\begin{array}{l}-- \\
-- \\
-- \\
-- \\
--\end{array}$ & $\begin{array}{l}-- \\
-- \\
-- \\
-- \\
--\end{array}$ & $\begin{array}{l}-- \\
-- \\
-- \\
-- \\
--\end{array}$ & $\begin{array}{l}-- \\
-- \\
-- \\
-- \\
--\end{array}$ & $\begin{array}{l}5.4 \\
5.1 \\
3.9 \\
-- \\
5.7\end{array}$ \\
\hline & $\begin{array}{r}4-28-71 \\
7-28-71 \\
9-21-71 \\
10-29-71 \\
1-26-72\end{array}$ & $\begin{array}{l}-\overline{26.0} \\
28.0 \\
22.0 \\
--\end{array}$ & $\begin{array}{r}194 \\
176 \\
90 \\
99 \\
138\end{array}$ & $\begin{array}{l}-- \\
6.5 \\
6.6 \\
5.6 \\
--\end{array}$ & $\begin{array}{l}-- \\
60 \\
32 \\
28 \\
--\end{array}$ & $\begin{array}{l}16 \\
15 \\
10 \\
13 \\
14\end{array}$ & $\begin{array}{l}58 \\
69 \\
25 \\
30 \\
59\end{array}$ & $\begin{array}{l}-- \\
-- \\
-- \\
-- \\
--\end{array}$ & $\begin{array}{l}-- \\
-- \\
-- \\
-- \\
--\end{array}$ & $\begin{array}{l}-- \\
-- \\
-- \\
-- \\
--\end{array}$ & $\begin{array}{l}-- \\
-- \\
-- \\
-- \\
--\end{array}$ & $\begin{array}{c}3 \\
1 \\
-- \\
-- \\
10\end{array}$ \\
\hline & $\begin{array}{r}3-17-72 \\
7-17-72 \\
10-26-72 \\
4-26-73 \\
8-13-73\end{array}$ & $\begin{array}{l}-- \\
-- \\
-- \\
-- \\
--\end{array}$ & $\begin{array}{l}120 \\
156 \\
181 \\
151 \\
180\end{array}$ & $\begin{array}{l}-- \\
-- \\
-- \\
--\end{array}$ & $\begin{array}{l}-- \\
-- \\
-- \\
-\end{array}$ & $\begin{array}{l}14 \\
20 \\
14 \\
15 \\
16\end{array}$ & $\begin{array}{l}33 \\
44 \\
60 \\
43 \\
50\end{array}$ & $\begin{array}{r}8 \\
17 \\
19 \\
13 \\
15\end{array}$ & $\begin{array}{r}3.4 \\
.4 \\
2.9 \\
2.5 \\
3.1\end{array}$ & $\begin{array}{c}1 \\
-- \\
9.4 \\
9.4 \\
11\end{array}$ & $\begin{array}{l}0 \\
3.1 \\
-- \\
2.2 \\
4.1\end{array}$ & $\begin{array}{l}-- \\
-- \\
-- \\
-- \\
--\end{array}$ \\
\hline & $\begin{array}{l}10-16-73 \\
12-20-73\end{array}$ & - & $\begin{array}{l}113 \\
165\end{array}$ & $\overline{-}$ & -- & $\begin{array}{l}16 \\
22\end{array}$ & $\begin{array}{l}29 \\
51\end{array}$ & $\begin{array}{r}8 \\
14\end{array}$ & $\begin{array}{l}2.1 \\
3.9\end{array}$ & ${ }_{11}^{8.1}$ & $\begin{array}{l}3.2 \\
8.5\end{array}$ & -- \\
\hline
\end{tabular}


Table 6a.--Water-quality data for ground-water Sites at the Rocky CReEk LANDFill

\begin{tabular}{|c|c|c|c|c|c|c|c|c|c|c|}
\hline STATION NUMBER & $\begin{array}{c}\text { DATE } \\
\text { OF } \\
\text { SAMPLE }\end{array}$ & $\begin{array}{l}\text { TEMPER- } \\
\text { ATURE } \\
\text { (UEG C) }\end{array}$ & $\begin{array}{l}\text { SPE - } \\
\text { CIFIC } \\
\text { CON- } \\
\text { UUCT- } \\
\text { ANCE } \\
\text { (MICRO- } \\
\text { MHOS) }\end{array}$ & $\begin{array}{l}\text { CHLO- } \\
\text { RIOE, } \\
\text { DIS- } \\
\text { SOLVED } \\
\text { (MG/L } \\
\text { AS CL) }\end{array}$ & $\begin{array}{c}\text { PH } \\
\text { (UNITS) }\end{array}$ & $\begin{array}{l}\text { ALKA- } \\
\text { LINITY } \\
\text { (MG/L } \\
\text { AS } \\
\text { CACO3) }\end{array}$ & $\begin{array}{l}\text { CALCIUM } \\
\text { UIS- } \\
\text { SOLVED } \\
\text { (MG/L } \\
\text { AS CA) }\end{array}$ & $\begin{array}{l}\text { MAGNE- } \\
\text { SIUM, } \\
\text { DIS- } \\
\text { SOLVED } \\
\text { (MG } \\
\text { AS MG) }\end{array}$ & $\begin{array}{l}\text { SOOIUM, } \\
\text { DIS- } \\
\text { SOLVED } \\
\text { (MG/L } \\
\text { AS NA) }\end{array}$ & $\begin{array}{l}\text { POTAS- } \\
\text { SIUM, } \\
\text { DIS- } \\
\text { SOLVEO } \\
\text { (MG/L } \\
\text { AS K) }\end{array}$ \\
\hline $\begin{array}{l}280219082342801 \\
280221082342901\end{array}$ & $\begin{array}{l}70-01-29 \\
70-10-23 \\
70-04-20 \\
70-10-23 \\
71-11-22\end{array}$ & $\begin{array}{l}23.5 \\
25.0 \\
21.5 \\
25.5 \\
--\end{array}$ & $\begin{array}{l}380 \\
376 \\
375 \\
382 \\
385\end{array}$ & $\begin{array}{l}10 \\
8.0 \\
10 \\
11 \\
10\end{array}$ & $\begin{array}{l}8.2 \\
7.5 \\
8.1 \\
8.1 \\
. .\end{array}$ & $\begin{array}{l}189 \\
230 \\
192 \\
192 \\
--\end{array}$ & $\begin{array}{l}69 \\
70 \\
38 \\
18 \\
.2\end{array}$ & $\begin{array}{l}3.8 \\
3.9 \\
1.9 \\
1.0 \\
--.\end{array}$ & $\begin{array}{l}7.1 \\
7.8 \\
4.5 \\
7.5 \\
.-\end{array}$ & $\begin{array}{l}.8 \\
.8 \\
.6 \\
.4 \\
.0\end{array}$ \\
\hline & $\begin{array}{l}71-12-27 \\
72-07-18 \\
72-10-25 \\
73-02-01 \\
73-04-23\end{array}$ & $\begin{array}{l}- \\
=- \\
--\end{array}$ & $\begin{array}{l}368 \\
360 \\
377 \\
375 \\
370\end{array}$ & $\begin{array}{l}10 \\
13 \\
8.5 \\
10 \\
13\end{array}$ & $\begin{array}{r}=- \\
\overline{--} \\
6.5 \\
7.7\end{array}$ & 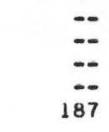 & $\begin{array}{l}\overline{-.} \\
70^{1.4} \\
69\end{array}$ & $\begin{array}{r}=- \\
-. \\
3.5 \\
3.9\end{array}$ & $\begin{array}{l}=- \\
90 \\
0.6 \\
6.7\end{array}$ & $\begin{array}{l}-. \\
-8 \\
.4 \\
.8\end{array}$ \\
\hline 280226082340701 & $\begin{array}{l}73-10-15 \\
73-12-20 \\
70-01-29 \\
72-07-17 \\
72-10-25\end{array}$ & $\begin{array}{r}-- \\
20.5 \\
-- \\
--\end{array}$ & $\begin{array}{r}379 \\
381 \\
81 \\
950 \\
81\end{array}$ & $\begin{array}{r}11 \\
7.0 \\
8.0 \\
3.0\end{array}$ & $\begin{array}{r}-- \\
0.4 \\
0- \\
--\end{array}$ & $\begin{array}{l}=- \\
28 \\
--\end{array}$ & $\begin{array}{c}71 \\
68 \\
12 \\
4.1\end{array}$ & $\begin{array}{r}3.5 \\
-. \\
-.9 \\
2.9 \\
1.8\end{array}$ & $\begin{array}{r}6.7 \\
-. . \\
.2 \\
4.6\end{array}$ & $\begin{array}{l}7.0 \\
- \\
\therefore \\
-\end{array}$ \\
\hline 280226082342401 & $\begin{array}{l}70-01-29 \\
70-03-30 \\
70-04-20 \\
70-06-01 \\
70-06-25\end{array}$ & $\begin{array}{l}21.0 \\
22.0 \\
23.0 \\
25.0 \\
25.0\end{array}$ & $\begin{array}{l}265 \\
212 \\
144 \\
136 \\
142\end{array}$ & $\begin{array}{l}17 \\
30 \\
19 \\
22 \\
19\end{array}$ & $\begin{array}{l}7.0 \\
=- \\
=- \\
6.6\end{array}$ & $\begin{array}{c}164 \\
-- \\
=- \\
62\end{array}$ & $\begin{array}{l}-- \\
=- \\
--\end{array}$ & $\begin{array}{l}=- \\
=- \\
=-\end{array}$ & $\begin{array}{l}\because \\
\because \\
\because \\
=\end{array}$ & $\begin{array}{l}= \\
=- \\
= \\
=\end{array}$ \\
\hline & $\begin{array}{l}70-07-15 \\
70-08-05 \\
70-09-18 \\
70-10-27 \\
70-12-03\end{array}$ & $\begin{array}{r}26.0 \\
30.0 \\
-. \\
25.0 \\
-.\end{array}$ & $\begin{array}{l}178 \\
216 \\
195 \\
192 \\
232\end{array}$ & $\begin{array}{l}19 \\
11 \\
11 \\
8.0 \\
25\end{array}$ & $\begin{array}{l}-- \\
6.4 \\
-- \\
6.4 \\
6.4\end{array}$ & $\begin{array}{l}10 \\
102 \\
178 \\
148\end{array}$ & $\begin{array}{l}- \\
\therefore \\
=- \\
--\end{array}$ & $\begin{array}{l}= \\
= \\
= \\
=\end{array}$ & $\begin{array}{l}\because \\
\therefore \\
\therefore\end{array}$ & $\begin{array}{l}= \\
\therefore \\
=\end{array}$ \\
\hline & $\begin{array}{l}71-01-14 \\
71-02-26 \\
71-03-31 \\
71-04-28 \\
71-06-15\end{array}$ & $\begin{array}{l}22.5 \\
23.0 \\
18.0 \\
24.0 \\
24.0\end{array}$ & $\begin{array}{l}208 \\
238 \\
222 \\
246 \\
264\end{array}$ & $\begin{array}{l}25 \\
30 \\
26 \\
22 \\
26\end{array}$ & $\begin{array}{l}6.6 \\
6.5 \\
6.6 \\
-- \\
6.2\end{array}$ & $\begin{array}{r}60 \\
-- \\
148 \\
-- \\
88\end{array}$ & $\begin{array}{l}-- \\
-- \\
--\end{array}$ & $\begin{array}{l}= \\
=- \\
= \\
=\end{array}$ & $\begin{array}{l}\because \\
\therefore \\
=\end{array}$ & $\begin{array}{l}- \\
\therefore \\
=\end{array}$ \\
\hline & $\begin{array}{l}71-07-28 \\
71-09-21 \\
71-10-29 \\
72-03-16 \\
72-07-17\end{array}$ & $\begin{array}{r}26.0 \\
-. \\
25.0 \\
--\end{array}$ & $\begin{array}{l}270 \\
400 \\
230 \\
210 \\
184\end{array}$ & $\begin{array}{l}29 \\
29 \\
27 \\
18 \\
17\end{array}$ & $\begin{array}{r}6.4 \\
6.2 \\
6.1 \\
-- \\
--\end{array}$ & $\begin{array}{r}144 \\
80 \\
76 \\
-- \\
--\end{array}$ & $\begin{array}{l}\overline{--} \\
24^{--}\end{array}$ & $\begin{array}{l}=- \\
=- \\
3.6 \\
7.3\end{array}$ & $\begin{array}{l}= \\
\overline{-} \\
40 \\
-0\end{array}$ & $\begin{array}{l}- \\
- \\
-1 \\
.2\end{array}$ \\
\hline 280226082342502 & $\begin{array}{l}72-10-25 \\
70-01-29 \\
70-03-30 \\
70-04-20 \\
70-06-01\end{array}$ & $\begin{array}{l}19.0 \\
21.0 \\
23.0 \\
25.0\end{array}$ & $\begin{array}{r}204 \\
92 \\
137 \\
237 \\
121\end{array}$ & $\begin{array}{l}17 \\
15 \\
14 \\
20 \\
14\end{array}$ & $\begin{array}{l}-. \\
6.9 \\
=- \\
--\end{array}$ & $\begin{array}{l}-- \\
20 \\
=- \\
--\end{array}$ & $\begin{array}{r}26 \\
=- \\
=-\end{array}$ & $\begin{array}{l}6.7 \\
=- \\
=- \\
=-\end{array}$ & $\begin{array}{r}10 \\
= \\
= \\
=\end{array}$ & $\begin{array}{l}- \\
\therefore \\
\therefore\end{array}$ \\
\hline & $\begin{array}{l}70-06-25 \\
70-07-15 \\
70-08-05 \\
70-09-18 \\
70-10-26\end{array}$ & $\begin{array}{l}26.0 \\
27.5 \\
28.5 \\
28.0 \\
26.0\end{array}$ & $\begin{array}{l}153 \\
178 \\
163 \\
-161\end{array}$ & $\begin{array}{r}9.0 \\
8.0 \\
11 \\
9.0\end{array}$ & $\begin{array}{l}7.0 \\
0.5 \\
6.5 \\
6.5 \\
6.6\end{array}$ & $\begin{array}{r}84 \\
-8 \\
86 \\
76 \\
100\end{array}$ & $=$ & $\begin{array}{l}\because \\
\because \\
\because\end{array}$ & $\begin{array}{l}\because \\
\because \\
\therefore\end{array}$ & $\begin{array}{l}\because \\
\because \\
=\end{array}$ \\
\hline & $\begin{array}{l}70-12-03 \\
71-01-14 \\
71-02-26 \\
71-03-30 \\
71-04-28\end{array}$ & $\begin{array}{l}23.0 \\
22.0 \\
22.5 \\
19.0 \\
25.5\end{array}$ & $\begin{array}{l}194 \\
139 \\
152 \\
133 \\
180\end{array}$ & $\begin{array}{l}10 \\
5.0 \\
11 \\
11 \\
13\end{array}$ & $\begin{array}{l}6.6 \\
6.5 \\
6.5 \\
6.7 \\
.-\end{array}$ & $\begin{array}{r}170 \\
87 \\
62 \\
88 \\
--\end{array}$ & $\begin{array}{l}= \\
=- \\
-\end{array}$ & $\begin{array}{l}= \\
= \\
= \\
=\end{array}$ & $\begin{array}{l}= \\
= \\
= \\
=\end{array}$ & $\begin{array}{l}= \\
\therefore \\
=\end{array}$ \\
\hline & $\begin{array}{l}71-07-27 \\
71-09-21 \\
71-10-29 \\
71-11-22 \\
72-01-27\end{array}$ & $\begin{array}{r}28.0 \\
-- \\
27.0 \\
=-\end{array}$ & $\begin{array}{l}177 \\
150 \\
249 \\
230 \\
185\end{array}$ & $\begin{array}{l}9.0 \\
14 \\
17 \\
14 \\
14\end{array}$ & $\begin{array}{l}6.5 \\
6.6 \\
6.6 \\
-- \\
--\end{array}$ & $\begin{array}{r}92 \\
80 \\
136 \\
-- \\
--\end{array}$ & $\begin{array}{l}= \\
=- \\
=\end{array}$ & $\begin{array}{l}= \\
= \\
= \\
=\end{array}$ & $\begin{array}{l}= \\
= \\
=\end{array}$ & $\begin{array}{l}- \\
\therefore \\
= \\
=\end{array}$ \\
\hline & $\begin{array}{l}72-01-27 \\
72-07-17 \\
72-10-25 \\
73-02-01 \\
73-03-16\end{array}$ & $\begin{array}{l}= \\
\ddot{z} \\
m\end{array}$ & $\begin{array}{l}412 \\
316 \\
225 \\
220\end{array}$ & $\begin{array}{l}21^{--} \\
14 \\
16 \\
16\end{array}$ & $\begin{array}{r}6.7 \\
-. \\
6.5 \\
-.\end{array}$ & $\begin{array}{l}95 \\
=- \\
=- \\
=-\end{array}$ & $\begin{array}{l}110^{--} \\
39 \\
21 \\
24\end{array}$ & $\begin{array}{l}24^{--} \\
5.0 \\
2.9 \\
22^{2.9}\end{array}$ & $\begin{array}{l}-.8 \\
9.8 \\
9.6 \\
3.5\end{array}$ & $\begin{array}{l}0 \\
2.0 \\
.4 \\
.1\end{array}$ \\
\hline 280226082342501 & $\begin{array}{l}73-04-26 \\
70-01-30 \\
70-04-20 \\
70-06-01 \\
70-06-25\end{array}$ & $\begin{array}{l}20.0 \\
29.0 \\
24.0 \\
29.5\end{array}$ & $\begin{array}{l}283 \\
278 \\
368 \\
340 \\
300\end{array}$ & $\begin{array}{l}14 \\
14 \\
17 \\
12 \\
12\end{array}$ & $\begin{array}{r}7.0 \\
\overrightarrow{7.9}\end{array}$ & $\begin{array}{l}178 \\
\overline{216} \\
\overline{216}\end{array}$ & $\begin{array}{r}30 \\
=- \\
=\end{array}$ & $\begin{array}{l}4.1 \\
=- \\
=- \\
=\end{array}$ & $\begin{array}{l}8.9 \\
- \\
=- \\
=-\end{array}$ & $\begin{array}{l}06 \\
\therefore- \\
\therefore\end{array}$ \\
\hline & $\begin{array}{l}70-07-15 \\
70-08-05 \\
70-09-18 \\
70-10-26 \\
70-12-03\end{array}$ & $\begin{array}{l}28.0 \\
27.0 \\
30.0 \\
26.5 \\
23.0\end{array}$ & $\begin{array}{l}364 \\
412 \\
-- \\
366 \\
382\end{array}$ & $\begin{array}{l}10 \\
11 \\
10^{-0} \\
10\end{array}$ & $\begin{array}{l}7.0 \\
8.0 \\
7.9 \\
7.6\end{array}$ & $\begin{array}{l}232 \\
-- \\
230 \\
228\end{array}$ & $=$ & $\begin{array}{l}\because \\
z \\
z\end{array}$ & $\begin{array}{l}= \\
= \\
=\end{array}$ & $\begin{array}{l}= \\
- \\
=\end{array}$ \\
\hline & $\begin{array}{l}71-01-14 \\
71-02-26 \\
71-03-31 \\
71-04-28 \\
71-06-15\end{array}$ & $\begin{array}{l}23.0 \\
22.0 \\
20.0 \\
24.5 \\
26.5\end{array}$ & $\begin{array}{l}380 \\
384 \\
378 \\
382 \\
365\end{array}$ & $\begin{array}{l}11 \\
9.0 \\
9.0 \\
9.0 \\
9.0\end{array}$ & $\begin{array}{l}7.7 \\
7.6 \\
7.8 \\
7.3\end{array}$ & $\begin{array}{l}232 \\
232 \\
234 \\
226\end{array}$ & $=$ & $\begin{array}{l}\because \\
\therefore \\
z\end{array}$ & $\begin{array}{l}= \\
\because \\
=\end{array}$ & $\begin{array}{l}\square \\
\therefore\end{array}$ \\
\hline & $\begin{array}{l}71-07-27 \\
71-10-29 \\
71-11-22 \\
72-07-18 \\
72-10-25\end{array}$ & $\begin{array}{r}27.0 \\
26.0 \\
=- \\
=-\end{array}$ & $\begin{array}{l}377 \\
272 \\
311 \\
370 \\
369\end{array}$ & $\begin{array}{l}9 \cdot 0 \\
15 \\
15 \\
14 \\
10\end{array}$ & $\begin{array}{l}7.5 \\
=- \\
=- \\
=-\end{array}$ & $\begin{array}{l}248 \\
=- \\
= \\
=\end{array}$ & $\begin{array}{l}-- \\
51 \\
87 \\
72\end{array}$ & $\begin{array}{l}= \\
5.6 \\
1.6 \\
6.2\end{array}$ & $\begin{array}{l}= \\
\because \\
0.8\end{array}$ & $\begin{array}{l}0 \\
5.5 \\
1.0 \\
1.4\end{array}$ \\
\hline
\end{tabular}


TABLE 6A, --WATER-Quality DATA FOR GROUND-WATER SITES AT THE ROCKY CREEK LANDFILL--CONTINUED

\begin{tabular}{|c|c|c|c|c|c|c|c|c|c|c|}
\hline STATION NUMBER & $\begin{array}{c}\text { OATE } \\
\text { OF } \\
\text { SAMPLE }\end{array}$ & $\begin{array}{l}\text { TEMPER- } \\
\text { ATURE } \\
\text { (UEG C) }\end{array}$ & $\begin{array}{l}\text { SPE- } \\
\text { CIF IC } \\
\text { CON- } \\
\text { OUCT- } \\
\text { ANEE } \\
\text { (MICRO- } \\
\text { MHOS) }\end{array}$ & $\begin{array}{l}\text { CHLO- } \\
\text { RIDE, } \\
\text { DIS- } \\
\text { SULVED } \\
\text { (MG/L } \\
\text { AS CL) }\end{array}$ & $\begin{array}{c}\text { PH } \\
\text { (UNITS) }\end{array}$ & $\begin{array}{l}\text { ALALA- } \\
\text { LINIYY } \\
\text { MMSL } \\
\text { AS } \\
\text { CACO3) }\end{array}$ & $\begin{array}{l}\text { CALCIUM } \\
\text { DIS- } \\
\text { SOLVED } \\
\text { (MGGL } \\
\text { AS CA }\end{array}$ & $\begin{array}{l}\text { MAGNE- } \\
\text { SIUM, } \\
\text { DIS- } \\
\text { SOLVED } \\
\text { (MG/L } \\
\text { AS MG) }\end{array}$ & $\begin{array}{l}\text { SOOUM, } \\
\text { DIS- } \\
\text { SOLED } \\
\text { (MG/ } \\
\text { AS NA) }\end{array}$ & $\begin{array}{l}\text { POTAS- } \\
\text { SIUM, } \\
\text { DIS- } \\
\text { SOLVEU } \\
\text { SMG L L } \\
\text { AS KI }\end{array}$ \\
\hline 280226082342501 & $\begin{array}{l}73-04-26 \\
73-08-09\end{array}$ & $=$ & $\begin{array}{l}380 \\
388\end{array}$ & $\begin{array}{l}6.8 \\
8.0\end{array}$ & $\because$ & $\because$ & $\begin{array}{l}66 \\
69\end{array}$ & $\begin{array}{l}5.6 \\
3.9\end{array}$ & $\begin{array}{l}7.6 \\
6.4\end{array}$ & $\begin{array}{r}1.1 \\
.0\end{array}$ \\
\hline 280226082342902 & $\begin{array}{l}73-12-19 \\
700-03-30 \\
70-04-20\end{array}$ & 20.0 & $\begin{array}{l}383 \\
100 \\
202\end{array}$ & $\begin{array}{l}8.4 \\
18\end{array}$ & $\begin{array}{l}=- \\
\because-\end{array}$ & $=$ & ${ }^{63}=$ & $\begin{array}{l}5.8 \\
--\end{array}$ & $\begin{array}{l}8.1 \\
-:-\end{array}$ & $\because 8$ \\
\hline $\begin{array}{l}280226082341202 \\
280226082341201\end{array}$ & $\begin{array}{l}70-06-02 \\
70-00-25 \\
70-07-14 \\
73-04-24 \\
73-04-25\end{array}$ & $\begin{array}{r}24.0 \\
260 \\
27.0 \\
-\because-\end{array}$ & $\begin{array}{l}108 \\
324 \\
416 \\
80 \\
420\end{array}$ & $\begin{array}{l}13 \\
17 \\
13 \\
5.7 \\
9.0\end{array}$ & $\begin{array}{l}z \\
\ddot{z} \\
5.2\end{array}$ & $\begin{array}{l}-z \\
-\because \\
--\end{array}$ & $\begin{array}{c}167 \\
167^{--} \\
73 \\
73\end{array}$ & $\begin{array}{l}z= \\
\because- \\
1.2 \\
5.8\end{array}$ & $\begin{array}{l}z= \\
z- \\
8.6 \\
8.2\end{array}$ & $\begin{array}{l}- \\
\because \\
\because 2\end{array}$ \\
\hline $28022608<342504$ & $\begin{array}{l}71-12-28 \\
72-01-26 \\
72-03-16 \\
772-07-19 \\
72-10-25\end{array}$ & $\begin{array}{l}= \\
z \\
z\end{array}$ & $\begin{array}{l}380 \\
370 \\
380 \\
382 \\
377\end{array}$ & $\begin{array}{l}9.0 \\
10 \\
10 \\
13 \\
7.2\end{array}$ & $\begin{array}{l}7.5 \\
\because \because \\
\ddot{-}\end{array}$ & $\begin{array}{r}\overline{-} \\
194 \\
\overline{-} \\
--\end{array}$ & $\begin{array}{l}68 \\
70- \\
78 \\
69\end{array}$ & $\begin{array}{r}122 \\
-- \\
3.8 \\
17 \\
3.9\end{array}$ & $\begin{array}{l}6.1 \\
--.5 \\
-- \\
0.3\end{array}$ & $\begin{array}{l}.6 \\
:-3 \\
: 1\end{array}$ \\
\hline 280227082343001 & $\begin{array}{l}73-04-23 \\
73-08-09 \\
73-10-15 \\
73-12-19 \\
70-01-29\end{array}$ & $\begin{array}{r}\because= \\
\because= \\
24.0\end{array}$ & $\begin{array}{l}387 \\
388 \\
384 \\
383 \\
378\end{array}$ & $\begin{array}{l}8.0 \\
8.0 \\
9.0 \\
8.4 \\
9.0\end{array}$ & $\begin{array}{l}= \\
= \\
8.0\end{array}$ & $\begin{array}{l}- \\
\because- \\
-\square \\
189\end{array}$ & $\begin{array}{l}67 \\
69 \\
72 \\
63 \\
69\end{array}$ & $\begin{array}{l}4.1 \\
3.9 \\
3.6 \\
5.8 \\
3.9\end{array}$ & $\begin{array}{l}7.4 \\
6.4 \\
6.4 \\
8.1 \\
6.0\end{array}$ & $\begin{array}{r}: 8 \\
: 8 \\
7: 0 \\
: 8 \\
.8\end{array}$ \\
\hline & $\begin{array}{l}70-03-30 \\
70-04-20 \\
70-00-01 \\
700-06-02 \\
70-06-25\end{array}$ & $\begin{array}{l}24.0 \\
24.0 \\
24.0 \\
24.0 \\
24.0\end{array}$ & $\begin{array}{l}348 \\
346 \\
--\end{array}$ & $\begin{array}{l}10^{--} \\
14^{--}\end{array}$ & $\begin{array}{c}= \\
\because \\
8.4\end{array}$ & $\begin{array}{l}= \\
\because z \\
220\end{array}$ & $\begin{array}{l}= \\
= \\
=\end{array}$ & $\begin{array}{l}= \\
= \\
=\end{array}$ & $\begin{array}{l}z \\
z \\
z\end{array}$ & $\begin{array}{l}\because \\
\because \\
\because\end{array}$ \\
\hline & $\begin{array}{l}70-07-14 \\
70-08-04 \\
70-09-18 \\
70-10-22 \\
70-12-03\end{array}$ & $\begin{array}{l}24.0 \\
24.0 \\
26.0 \\
24.0 \\
22.0\end{array}$ & $\begin{array}{l}368 \\
391 \\
388 \\
360 \\
364\end{array}$ & $\begin{array}{l}10 \\
9.0 \\
13 \\
9.0 \\
10\end{array}$ & $\begin{array}{l}7.3 \\
7.3 \\
7.3 \\
7.1\end{array}$ & $\begin{array}{l}22- \\
229 \\
190 \\
226\end{array}$ & $\begin{array}{l}=- \\
70- \\
70-\end{array}$ & $\begin{array}{l}= \\
\ddot{-} \\
3.9\end{array}$ & $\begin{array}{l}= \\
-\square \\
6.3 \\
--\end{array}$ & $\begin{array}{l}\because- \\
\because-8\end{array}$ \\
\hline & $\begin{array}{l}71-01-14 \\
71-02-24 \\
711-03-31 \\
711-04-28 \\
71-00-15\end{array}$ & $\begin{array}{l}24.0 \\
26.0 \\
23.0 \\
25.0 \\
24.0\end{array}$ & $\begin{array}{l}374 \\
368 \\
368 \\
372 \\
382\end{array}$ & $\begin{array}{r}8.0 \\
9.0 \\
9.0 \\
9.0 \\
10\end{array}$ & $\begin{array}{l}7.5 \\
7.4 \\
7.3 \\
7.2\end{array}$ & $\begin{array}{l}230 \\
226 \\
230 \\
-- \\
230\end{array}$ & $\begin{array}{l}= \\
= \\
=-\end{array}$ & $\begin{array}{l}=- \\
=- \\
=-\end{array}$ & $\begin{array}{l}\because \\
\because \\
\because\end{array}$ & $\begin{array}{l}= \\
z \\
z\end{array}$ \\
\hline & $\begin{array}{l}71-07-28 \\
71-09-22 \\
71-10-28 \\
7111-22 \\
71-12-22\end{array}$ & $\begin{array}{r}23.5 \\
24.0 \\
23.0 \\
-\therefore\end{array}$ & $\begin{array}{l}370 \\
370 \\
355 \\
317 \\
--\end{array}$ & $\begin{array}{r}9.0 \\
8.0 \\
11 \\
9.4 \\
0 .-\end{array}$ & $\begin{array}{l}7.2 \\
7: 3 \\
7: 1 \\
\because-\end{array}$ & $\begin{array}{r}248 \\
230 \\
228 \\
-\because\end{array}$ & $\begin{array}{l}= \\
=- \\
55\end{array}$ & $\begin{array}{l}= \\
= \\
z .2\end{array}$ & $\begin{array}{l}z \\
z \\
5.0\end{array}$ & $\begin{array}{l}= \\
= \\
= \\
2\end{array}$ \\
\hline & $\begin{array}{l}71-12-27 \\
72-02-01 \\
722-03-17 \\
72-06-20 \\
72-07-18\end{array}$ & $\begin{array}{l}- \\
\because z \\
--\end{array}$ & $\begin{array}{l}376 \\
370 \\
370 \\
362 \\
379\end{array}$ & $\begin{array}{r}9.0 \\
9.2 \\
9.2 \\
13\end{array}$ & $\begin{array}{r}6.9 \\
7.7 \\
- \\
\hdashline-\end{array}$ & $\begin{array}{l}214 \\
=- \\
=- \\
--\end{array}$ & $\begin{array}{c}=- \\
170^{--} \\
67 \\
80\end{array}$ & $\begin{array}{c}-= \\
10 \\
5.0 \\
6.2\end{array}$ & $\begin{array}{l}-z \\
1.5 \\
6.0 \\
-.\end{array}$ & $\begin{array}{l}-2 \\
.5 \\
.7 \\
.1\end{array}$ \\
\hline & $\begin{array}{l}72-10-25 \\
73-02-01 \\
73-04-23 \\
73-08-09 \\
73-10-15\end{array}$ & $\begin{array}{l}z \\
z \\
z\end{array}$ & $\begin{array}{r}370 \\
370 \\
382 \\
379 \\
379\end{array}$ & $\begin{array}{l}8.0 \\
10 \\
8.0 \\
10 \\
10\end{array}$ & $\begin{array}{l}7 \ddot{7} \\
7.7 \\
\because-\end{array}$ & $\begin{array}{r}- \\
18- \\
-= \\
--\end{array}$ & $\begin{array}{l}67 \\
69 \\
67 \\
70 \\
69\end{array}$ & $\begin{array}{l}4.0 \\
3.9 \\
4.01 \\
4.1 \\
3.7\end{array}$ & $\begin{array}{l}6.0 \\
6.0 \\
7.4 \\
6.3 \\
6.3\end{array}$ & $\begin{array}{r}.7 \\
0 \\
09 \\
0.8 \\
8.0\end{array}$ \\
\hline 280228082335901 & $\begin{array}{l}73-12-19 \\
72-07-17 \\
772-10-26 \\
73-02-01 \\
73-04-26\end{array}$ & $\begin{array}{l}z \\
z \\
z\end{array}$ & $\begin{array}{r}378 \\
149 \\
140 \\
115 \\
95\end{array}$ & $\begin{array}{l}8.7 \\
19 \\
18 \\
15 \\
11\end{array}$ & $\begin{array}{l}= \\
= \\
=\end{array}$ & $\begin{array}{l}= \\
\because \\
z\end{array}$ & $\begin{array}{l}67 \\
220 \\
270 \\
210 \\
110\end{array}$ & $\begin{array}{r}5.0 \\
2.4 \\
119 \\
1.5 \\
.9\end{array}$ & $\begin{array}{r}10 \\
5 .- \\
5.4 \\
8.0\end{array}$ & $\begin{array}{r}2.2 \\
.2 \\
-.4 \\
.4\end{array}$ \\
\hline 280228082341201 & $\begin{array}{l}72-07-17 \\
72-10-26 \\
73-02-01 \\
73-02-020 \\
73-08-13\end{array}$ & $\begin{array}{l}z \\
z \\
z\end{array}$ & $\begin{array}{l}347 \\
355 \\
350 \\
377 \\
112\end{array}$ & $\begin{array}{l}14 \\
9.5 \\
11 \\
10^{11}\end{array}$ & $\begin{array}{l}\because \because \\
\because \because 9 \\
\because-9\end{array}$ & $\begin{array}{l}\because \\
\because \\
\because\end{array}$ & $\begin{array}{l}71 \\
60 \\
66 \\
66 \\
8.7\end{array}$ & $\begin{array}{l}1.7 \\
4.5 \\
4.9 \\
4.5 \\
2.5\end{array}$ & $\begin{array}{l}6 .- \\
6.4 \\
6.3 \\
7.4 \\
7.7\end{array}$ & $\begin{array}{r}1.0 \\
.-8 \\
.8 \\
.4\end{array}$ \\
\hline 280228082342601 & $\begin{array}{l}73-10-17 \\
73-12-20 \\
70-01-30 \\
700-03-30 \\
70-04-20\end{array}$ & $\begin{array}{r}z- \\
18.5 \\
22.0 \\
24.0\end{array}$ & $\begin{array}{r}60 \\
76 \\
500 \\
458 \\
450\end{array}$ & $\begin{array}{l}10 \\
80.8 \\
30 \\
28 \\
29\end{array}$ & $\begin{array}{l}\ddot{-} \\
7.7 \\
\because-\end{array}$ & $\begin{array}{r}-= \\
133 \\
--\end{array}$ & $\begin{array}{r}3.5 \\
88.4 \\
68 \\
=-\end{array}$ & $\begin{array}{l}1.0 \\
2.0 \\
9.7 \\
--.\end{array}$ & $\begin{array}{r}6.0 \\
8.2 \\
18 \\
=-\end{array}$ & $\begin{array}{l}: 8 \\
: 8 \\
6.6 \\
\because-\end{array}$ \\
\hline & $\begin{array}{l}70-06-01 \\
700-06-02 \\
70-06-25 \\
700-07-15 \\
70-08-05\end{array}$ & $\begin{array}{l}24.0 \\
23.5 \\
25.0 \\
27.0 \\
26.0\end{array}$ & $\begin{array}{l}416 \\
139 \\
370 \\
434 \\
431\end{array}$ & $\begin{array}{l}25 \\
11 \\
22 \\
20 \\
21\end{array}$ & $\begin{array}{l}\because \\
= \\
=\end{array}$ & $\begin{array}{l}\because \\
\because \\
z\end{array}$ & $\begin{array}{l}= \\
= \\
=\end{array}$ & $\begin{array}{l}= \\
= \\
=\end{array}$ & $\begin{array}{l}- \\
z \\
-\end{array}$ & $\begin{array}{l}- \\
z \\
-=\end{array}$ \\
\hline & $\begin{array}{l}70-09-18 \\
70-10-23 \\
70-12-04 \\
71-01-13 \\
71-02-26\end{array}$ & $\begin{array}{l}28.0 \\
24.5 \\
23.0 \\
21.0 \\
21.0\end{array}$ & $\begin{array}{l}420 \\
415 \\
368 \\
400 \\
370\end{array}$ & $\begin{array}{l}17 \\
17 \\
12 \\
13 \\
10\end{array}$ & $\begin{array}{l}\ddot{-8} \\
\ddot{-} \\
=-\end{array}$ & $\begin{array}{r}197 \\
\because= \\
\because-\end{array}$ & $\begin{array}{l}73^{--} \\
70^{--}\end{array}$ & $\begin{array}{l}5.7 \\
5.7 \\
2.5 \\
--\end{array}$ & $\begin{array}{l}11-- \\
8.6\end{array}$ & $\begin{array}{l}3 . \overline{-} \\
2 . \overline{1}\end{array}$ \\
\hline & $\begin{array}{l}71-03-30 \\
711-04-28 \\
711-06-14 \\
711-07-28 \\
11-09-22\end{array}$ & $\begin{array}{l}20.0 \\
22.0 \\
23.5 \\
25.0 \\
25.5\end{array}$ & $\begin{array}{l}390 \\
402 \\
390 \\
383 \\
382\end{array}$ & $\begin{array}{l}10 \\
10 \\
10 \\
11 \\
10\end{array}$ & $\begin{array}{l}= \\
= \\
=\end{array}$ & $\begin{array}{l}z \\
z \\
z\end{array}$ & $\begin{array}{l}= \\
= \\
=\end{array}$ & $\begin{array}{l}= \\
= \\
=\end{array}$ & $\begin{array}{l}z \\
z \\
z\end{array}$ & $\begin{array}{l}= \\
z \\
z \\
=\end{array}$ \\
\hline
\end{tabular}




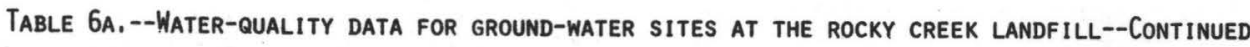

\begin{tabular}{|c|c|c|c|c|c|c|c|c|c|c|}
\hline STATION NUMEER & $\begin{array}{c}\text { DATE } \\
\text { OF } \\
\text { SAMPLE }\end{array}$ & $\begin{array}{l}\text { TEMPER- } \\
\text { ATURE } \\
\text { (DEG C) }\end{array}$ & $\begin{array}{l}\text { SPE- } \\
\text { CIFIC } \\
\text { CON- } \\
\text { DUCT- } \\
\text { ANCE } \\
\text { (MICRO- } \\
\text { MHOS) }\end{array}$ & $\begin{array}{l}\text { CHLO- } \\
\text { KIDE, } \\
\text { UIS- } \\
\text { SOLVEU } \\
\text { (MG } \\
\text { AS (LL) }\end{array}$ & $\begin{array}{c}P H \\
\text { (UNITS) }\end{array}$ & $\begin{array}{l}\text { ALKA- } \\
\text { LINITY } \\
\text { (MG/L } \\
\text { AS } \\
\text { CACO3) }\end{array}$ & $\begin{array}{l}\text { CALCIUMA } \\
\text { DIS- } \\
\text { SOLVED } \\
\text { (MG/L } \\
\text { AS CA) }\end{array}$ & $\begin{array}{l}\text { MAGNE- } \\
\text { SIUM, } \\
\text { DIS- } \\
\text { SOLVED } \\
\text { (MG/L } \\
\text { AS MG) }\end{array}$ & $\begin{array}{l}\text { SOUIUM, } \\
\text { UIS- } \\
\text { SOLVED } \\
\text { (MG/L } \\
\text { AS NA) }\end{array}$ & $\begin{array}{l}\text { POTAS- } \\
\text { SIUM, } \\
\text { DIS- } \\
\text { SOLVED } \\
\text { (MG L } \\
\text { AS K) }\end{array}$ \\
\hline \multirow[t]{2}{*}{280228082342601} & $\begin{array}{l}71-10-29 \\
71-11-22 \\
71-12-28 \\
72-07-19 \\
72-10-25\end{array}$ & $\begin{array}{l}= \\
=- \\
= \\
=\end{array}$ & $\begin{array}{l}368 \\
312 \\
388 \\
386 \\
377\end{array}$ & $\begin{array}{c}11 \\
9.5 \\
9.0 \\
12 \\
9.0\end{array}$ & $\begin{array}{l}= \\
\therefore \\
=\end{array}$ & $\begin{array}{l}= \\
\ddot{-} \\
=\end{array}$ & ${ }_{54}^{78^{--}}$ & ${ }_{4.1}^{1.3}$ & $\begin{array}{r}-- \\
5.0 \\
0.6\end{array}$ & $\begin{array}{l}\because-0 \\
\because 0 \\
1.0\end{array}$ \\
\hline & $\begin{array}{l}73-02-01 \\
73-04-23 \\
73-08-09 \\
73-10-17 \\
73-12-19\end{array}$ & $\begin{array}{l}= \\
-\infty \\
=\end{array}$ & $\begin{array}{l}940 \\
395 \\
378 \\
360 \\
396\end{array}$ & $\begin{array}{c}11 \\
8.7 \\
10 \\
9.0 \\
7.6\end{array}$ & $\begin{array}{l}= \\
= \\
= \\
=\end{array}$ & 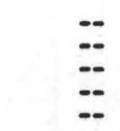 & $\begin{array}{l}76 \\
70 \\
67 \\
67 \\
68\end{array}$ & $\begin{array}{l}10 \\
4.2 \\
3.9 \\
3.2 \\
4.9\end{array}$ & $\begin{array}{l}6.4 \\
7.6 \\
6.8 \\
6.2 \\
7.3\end{array}$ & $\begin{array}{l}2.5 \\
1.1 \\
.4 \\
1.0 \\
2.8\end{array}$ \\
\hline \multirow[t]{3}{*}{280228082342602} & $\begin{array}{l}70-01-30 \\
70-0 j-30 \\
70-04-20 \\
70-06-01 \\
70-06-02\end{array}$ & $\begin{array}{l}16.0 \\
21.0 \\
23.0 \\
24.0 \\
25.5\end{array}$ & $\begin{array}{r}212 \\
178 \\
241 \\
152 \\
52\end{array}$ & $\begin{array}{l}10 \\
13 \\
13 \\
14 \\
10\end{array}$ & $\begin{array}{l}6.0 \\
=- \\
= \\
=\end{array}$ & $\begin{array}{l}86 \\
=- \\
=- \\
=-\end{array}$ & $\begin{array}{l}31 \\
= \\
= \\
=\end{array}$ & $\begin{array}{l}2.2 \\
=- \\
=- \\
=\end{array}$ & $\begin{array}{l}3.0 \\
-- \\
a \\
0\end{array}$ & $\begin{array}{l}\therefore \\
\therefore- \\
\therefore-\end{array}$ \\
\hline & $\begin{array}{l}70-06-25 \\
70-07-15 \\
70-08-05 \\
70-09-18 \\
71-02-26\end{array}$ & $\begin{array}{l}25.0 \\
26.0 \\
28.0 \\
27.3 \\
20.5\end{array}$ & $\begin{array}{l}145 \\
157 \\
204 \\
164 \\
198\end{array}$ & $\begin{array}{l}9.0 \\
8.0 \\
11 \\
10 \\
15\end{array}$ & $\begin{array}{l}= \\
\because \\
=\end{array}$ & 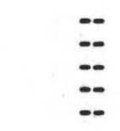 & $\begin{array}{l}= \\
\ddot{-} \\
=\end{array}$ & $\begin{array}{l}= \\
= \\
= \\
=\end{array}$ & 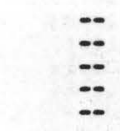 & $\ddot{-}=$ \\
\hline & $\begin{array}{l}71-09-22 \\
71-10-29 \\
73-04-23 \\
70-01-30 \\
70-03-30\end{array}$ & $\begin{array}{r}32.0 \\
=- \\
18.0 \\
21.0\end{array}$ & $\begin{array}{l}278 \\
328 \\
340 \\
312 \\
302\end{array}$ & $\begin{array}{l}11 \\
14 \\
6.3 \\
10^{\circ} \\
13\end{array}$ & $\begin{array}{l}= \\
=- \\
7.1\end{array}$ & $\begin{array}{c}a \\
151 \\
--\end{array}$ & $\begin{array}{l}= \\
60^{-.} \\
49 \\
-.\end{array}$ & $\begin{array}{l}= \\
3.5 \\
3.0 \\
--\end{array}$ & $\begin{array}{l}\ldots \\
7.6 \\
6.5 \\
-.\end{array}$ & $\begin{array}{l}- \\
-.8 \\
1.2 \\
-\square\end{array}$ \\
\hline \multirow{5}{*}{280228082342603} & $\begin{array}{l}70-03-31 \\
70-04-20 \\
70-06-01 \\
70-06-25 \\
70-07-15\end{array}$ & $\begin{array}{l}-.0 \\
23.0 \\
23.0 \\
24.0 \\
25.5\end{array}$ & $\begin{array}{l}305 \\
321 \\
299 \\
344\end{array}$ & $\begin{array}{l}8.0 \\
12 \\
13 \\
10 \\
9.0\end{array}$ & $\begin{array}{l}= \\
=- \\
0.0\end{array}$ & $\begin{array}{l}-- \\
209 \\
=-\end{array}$ & $\begin{array}{l}= \\
= \\
=\end{array}$ & $\begin{array}{l}= \\
= \\
= \\
=\end{array}$ & $\begin{array}{l}= \\
= \\
=\end{array}$ & $\begin{array}{l}\ldots \\
\therefore \\
\equiv \\
\end{array}$ \\
\hline & $\begin{array}{l}70-08-05 \\
70-09-18 \\
70-10-23 \\
70-12-04 \\
71-01-13\end{array}$ & $\begin{array}{l}25.0 \\
28.0 \\
24.5 \\
23.5 \\
22.0\end{array}$ & $\begin{array}{l}349 \\
348 \\
341 \\
422 \\
340\end{array}$ & $\begin{array}{c}10 \\
10 \\
9.0 \\
16 \\
8.0\end{array}$ & $\begin{array}{l}7.2 \\
7.3 \\
7.2 \\
7.3 \\
7.3\end{array}$ & $\begin{array}{l}218 \\
216 \\
220 \\
210 \\
216\end{array}$ & $65^{--}$ & $\begin{array}{l}-2 \\
3.5 \\
3.2\end{array}$ & $\begin{array}{l}-. \\
6.1 \\
5.7\end{array}$ & $\begin{array}{l}-. \\
.0 \\
.5 \\
.1\end{array}$ \\
\hline & $\begin{array}{l}71-02-26 \\
71-03-30 \\
71-04-28 \\
71-06-14 \\
71-07-28\end{array}$ & $\begin{array}{l}21.5 \\
20.0 \\
22.0 \\
23.0 \\
25.0\end{array}$ & $\begin{array}{l}358 \\
328 \\
352 \\
344 \\
348\end{array}$ & $\begin{array}{l}9.0 \\
9.0 \\
9.0 \\
8.0 \\
8.0\end{array}$ & $\begin{array}{l}7.1 \\
7.3 \\
7.1 \\
6.9\end{array}$ & $\begin{array}{l}222 \\
214 \\
216 \\
220\end{array}$ & $\begin{array}{l}= \\
= \\
=\end{array}$ & $\begin{array}{l}= \\
= \\
=\end{array}$ & $=$ & 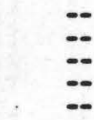 \\
\hline & $\begin{array}{l}71-09-22 \\
71-10-28 \\
71-10-29 \\
71-11-22 \\
71-12-28\end{array}$ & $\begin{array}{r}28.0 \\
=- \\
=\end{array}$ & $\begin{array}{l}368 \\
342 \\
310 \\
318\end{array}$ & $\begin{array}{r}9.0 \\
14 \\
9.5 \\
9.0\end{array}$ & $\begin{array}{l}7.0 \\
-.6 \\
-0 \\
7.6\end{array}$ & $\begin{array}{r}272 \\
60 \\
220 \\
-- \\
218\end{array}$ & $\begin{array}{c}=- \\
59^{--}\end{array}$ & $\begin{array}{l}= \\
\overline{3.2} \\
-\end{array}$ & $\ddot{0}$ & $\begin{array}{l}-0 \\
-0 \\
-0\end{array}$ \\
\hline & $\begin{array}{l}72-03-16 \\
72-07-19 \\
72-10-26 \\
73-02-01 \\
73-04-23\end{array}$ & 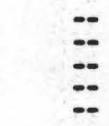 & $\begin{array}{l}330 \\
339 \\
336 \\
340 \\
340\end{array}$ & $\begin{array}{c}9.6 \\
13 \\
7.4 \\
10 \\
6.3\end{array}$ & $\begin{array}{l}= \\
7.6\end{array}$ & 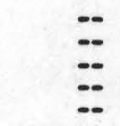 & $\begin{array}{l}60 \\
78 \\
65 \\
59 \\
60\end{array}$ & $\begin{array}{l}7.6 \\
6.3 \\
3.4 \\
3.2 \\
3.5\end{array}$ & $\begin{array}{l}1.0 \\
6.4 \\
6.4 \\
7.6\end{array}$ & $\begin{array}{l}.4 \\
.4 \\
.7 \\
.7 \\
.8\end{array}$ \\
\hline \multirow[t]{6}{*}{$\begin{array}{l}280228082342701 \\
280230082340501 \\
280231082343603\end{array}$} & $\begin{array}{l}71-10-28 \\
72-01-27 \\
72-03-16 \\
73-02-01 \\
70-01-13\end{array}$ & 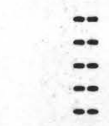 & $\begin{array}{r}4200 \\
182 \\
4320 \\
360 \\
63\end{array}$ & $\begin{array}{l}270 \\
9.0 \\
325 \\
19 \\
8.4\end{array}$ & $\begin{array}{l}7.1 \\
=.5 \\
7.3 \\
6.5\end{array}$ & $\begin{array}{l}a \\
\ddot{12}\end{array}$ & $\begin{array}{c}86 \\
120^{--} \\
-0\end{array}$ & ${ }^{54}$ & ${ }^{241}=$ & $170^{145}$ \\
\hline & $\begin{array}{l}70-02-04 \\
70-03-31 \\
70-04-23 \\
70-06-15 \\
70-06-16\end{array}$ & $\begin{array}{l}19.5 \\
23.0 \\
23.0 \\
25.0 \\
--\end{array}$ & $\begin{array}{r}135 \\
120 \\
93 \\
100 \\
-0\end{array}$ & $\begin{array}{l}6.5 \\
8.0 \\
7.0 \\
6.5 \\
. .0\end{array}$ & $\begin{array}{r}6.8 \\
-: \\
7.0 \\
6.5\end{array}$ & $\begin{array}{r}52 \\
6 \\
-1 \\
-2\end{array}$ & $\begin{array}{l}11 \\
-.5 \\
0.5\end{array}$ & $\begin{array}{l}1.0 \\
0 \\
1.2 \\
-.\end{array}$ & $\begin{array}{r}2.7 \\
0 . \\
2.5 \\
--\end{array}$ & $\begin{array}{r}2.3 \\
-. \\
2.7 \\
-.\end{array}$ \\
\hline & $\begin{array}{l}70-07-14 \\
70-08-04 \\
70-09-17 \\
70-10-22 \\
70-12-03\end{array}$ & $\begin{array}{l}26.0 \\
27.0 \\
25.5 \\
23.0\end{array}$ & $\begin{array}{r}61 \\
126 \\
62 \\
103 \\
69\end{array}$ & $\begin{array}{l}6.0 \\
7.0 \\
7.0 \\
6.0 \\
7.0\end{array}$ & $\begin{array}{l}-.5 \\
6.5 \\
6.5 \\
6.2 \\
6.0\end{array}$ & $\begin{array}{r}100 \\
45 \\
69 \\
34\end{array}$ & $=$ & $\begin{array}{l}\ddot{-} \\
\because 9\end{array}$ & $\begin{array}{l}0 \\
0.4 \\
0\end{array}$ & 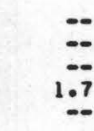 \\
\hline & $\begin{array}{l}71-01-14 \\
71-02-24 \\
71=03-31 \\
71=04-28 \\
71=06-15\end{array}$ & $\begin{array}{l}24.5 \\
22.0 \\
20.5 \\
26.0 \\
24.0\end{array}$ & $\begin{array}{l}68 \\
55 \\
44 \\
50 \\
58\end{array}$ & $\begin{array}{l}8.0 \\
7.0 \\
6.0 \\
5.0 \\
5.0\end{array}$ & $\begin{array}{l}6.3 \\
6.1 \\
6.1 \\
-5 \\
5.9\end{array}$ & $\begin{array}{l}24 \\
38 \\
16 \\
16\end{array}$ & $\begin{array}{l}= \\
= \\
-\end{array}$ & $\begin{array}{l}= \\
= \\
=\end{array}$ & $\begin{array}{l}a \\
\therefore \\
=\end{array}$ & $=$ \\
\hline & $\begin{array}{l}71-07-28 \\
71-09-21 \\
71-10-28 \\
71-11-22 \\
71-12-27\end{array}$ & \begin{tabular}{r}
30.0 \\
\hdashline \\
$=-$
\end{tabular} & $\begin{array}{r}76 \\
112 \\
65 \\
156 \\
105\end{array}$ & $\begin{array}{l}7.0 \\
6.0 \\
7.0 \\
9.0 \\
8.0\end{array}$ & $\begin{array}{l}6.3 \\
5.5 \\
-.5 \\
6.2\end{array}$ & $\begin{array}{l}76 \\
-\therefore \\
\ddot{40}\end{array}$ & 20 & $\begin{array}{l}\square \\
\square \\
\square .4 \\
-.\end{array}$ & $\bar{a}$ & $\begin{array}{l}0 \\
\ddot{1.1}\end{array}$ \\
\hline & $\begin{array}{l}72-03-16 \\
73-04-24 \\
73-08-09 \\
73-10-15 \\
73-12-20\end{array}$ & $\begin{array}{l}= \\
\equiv \\
-\infty\end{array}$ & $\begin{array}{r}110 \\
57 \\
68 \\
54 \\
63\end{array}$ & $\begin{array}{l}7.0 \\
1.0 \\
1.0 \\
4.5 \\
9.2\end{array}$ & 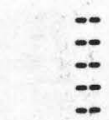 & $\begin{array}{l}= \\
= \\
=\end{array}$ & $\begin{array}{l}17 \\
46 \\
6.8 \\
4.1 \\
7.6\end{array}$ & $\begin{array}{l}5.0 \\
1.3 \\
1.3 \\
1.1 \\
.9\end{array}$ & $\begin{array}{l}.0 \\
2.3 \\
2.7 \\
2.2 \\
6.3\end{array}$ & $\begin{array}{l}1.2 \\
1.9 \\
1.5 \\
1.7 \\
2.2\end{array}$ \\
\hline
\end{tabular}




\begin{tabular}{|c|c|c|c|c|c|c|c|c|c|c|}
\hline STATION NUMBEK & $\begin{array}{c}\text { DATE } \\
\text { OF } \\
\text { SAMPLE }\end{array}$ & $\begin{array}{l}\text { TEMPER- } \\
\text { ATURE } \\
\text { (DEG C) }\end{array}$ & $\begin{array}{l}\text { SPE- } \\
\text { CIFIC } \\
\text { CON- } \\
\text { DUCT- } \\
\text { ANCE } \\
\text { (MICKO- } \\
\text { MHOS) }\end{array}$ & $\begin{array}{l}\text { CHLO- } \\
\text { RIDE, } \\
\text { DIS- } \\
\text { SOLVED } \\
\text { (MG } \\
\text { AS CL) }\end{array}$ & $\begin{array}{c}P H \\
\text { (UNITS) }\end{array}$ & $\begin{array}{l}\text { ALKA- } \\
\text { LINITY } \\
\text { (MG/L } \\
\text { AS } \\
\text { CACO3) }\end{array}$ & $\begin{array}{l}\text { CALCIUM } \\
\text { DIS- } \\
\text { SOLVED } \\
\text { (MG/L } \\
\text { AS CA) }\end{array}$ & $\begin{array}{l}\text { MAGNE- } \\
\text { SIUM, } \\
\text { UIS- } \\
\text { SOLVED } \\
\text { (MG/L } \\
\text { AS MG) }\end{array}$ & $\begin{array}{l}\text { SOUIUM, } \\
\text { OIS- } \\
\text { SOLVED } \\
\text { (MG/L } \\
\text { AS NA) }\end{array}$ & $\begin{array}{l}\text { POTAS- } \\
\text { SIUM, } \\
\text { OIS- } \\
\text { SOLVED } \\
\text { (MG/L } \\
\text { AS K) }\end{array}$ \\
\hline \multirow[t]{6}{*}{280231082343602} & $\begin{array}{l}70-02-04 \\
70-03-31 \\
70-04-23 \\
70-06-15 \\
70-06-25\end{array}$ & $\begin{array}{l}19.0 \\
23.0 \\
23.0 \\
24.0 \\
25.0\end{array}$ & $\begin{array}{l}164 \\
180 \\
178 \\
180 \\
148\end{array}$ & $\begin{array}{l}10 \\
10 \\
11 \\
14 \\
9.0\end{array}$ & $\begin{array}{r}7.0 \\
=- \\
8.0 \\
7.8\end{array}$ & $\begin{array}{l}54 \\
-2 \\
-2 \\
66 \\
84\end{array}$ & 23 & $\begin{array}{r}2.4 \\
-- \\
1.9 \\
--\end{array}$ & $\begin{array}{r}5.7 \\
-- \\
-- \\
b .4 \\
--\end{array}$ & $\begin{array}{r}2.4 \\
0 . \\
2.1 \\
--\end{array}$ \\
\hline & $\begin{array}{l}70-07-14 \\
70-08-04 \\
70-09-17 \\
70-12-03 \\
71-01-14\end{array}$ & $\begin{array}{l}25.9 \\
26.0 \\
27.2 \\
23.0 \\
24.0\end{array}$ & $\begin{array}{r}158 \\
63 \\
128 \\
137 \\
49\end{array}$ & $\begin{array}{l}9.0 \\
5.4 \\
8.0 \\
9.0 \\
4.0\end{array}$ & $\begin{array}{l}-. \\
6.4 \\
6.6 \\
6.7 \\
6.6\end{array}$ & $\begin{array}{r}-- \\
40 \\
66 \\
140 \\
103\end{array}$ & $\begin{array}{l}=- \\
=- \\
=-\end{array}$ & $\begin{array}{l}=- \\
=- \\
=-\end{array}$ & $\begin{array}{l}= \\
=- \\
=- \\
=-\end{array}$ & $\begin{array}{l}= \\
=- \\
=-\end{array}$ \\
\hline & $\begin{array}{l}71-02-24 \\
71-03-31 \\
71-04-28 \\
71-06-15 \\
71-07-28\end{array}$ & $\begin{array}{l}22.5 \\
21.0 \\
24.0 \\
24.0 \\
26.0\end{array}$ & $\begin{array}{r}92 \\
111 \\
107 \\
139 \\
100\end{array}$ & $\begin{array}{r}14 \\
7.0 \\
7.0 \\
7.0 \\
7.0\end{array}$ & $\begin{array}{l}6.4 \\
6.5 \\
-.- \\
6.4 \\
6.6\end{array}$ & $\begin{array}{r}144 \\
62 \\
-64 \\
64 \\
72\end{array}$ & $\begin{array}{l}-- \\
=- \\
--\end{array}$ & $\begin{array}{l}-- \\
=- \\
=-\end{array}$ & $\begin{array}{l}-- \\
=- \\
=-\end{array}$ & $\begin{array}{l}= \\
=- \\
=-\end{array}$ \\
\hline & $\begin{array}{l}71-09-21 \\
71-10-28 \\
71-11-22 \\
71-12-27 \\
72-01-26\end{array}$ & $\begin{array}{l}=- \\
=- \\
=- \\
=\end{array}$ & $\begin{array}{r}310 \\
202 \\
85\end{array}$ & $\begin{array}{c}8.0 \\
10 \\
8.0 \\
8.0 \\
13\end{array}$ & $\begin{array}{l}-- \\
6.8 \\
-- \\
6.6 \\
6.4\end{array}$ & $\begin{array}{r}225 \\
-- \\
196 \\
46\end{array}$ & 53 & $\begin{array}{l}-- \\
-- \\
4.4 \\
4.4 \\
--\end{array}$ & $\begin{array}{l}-- \\
-- \\
5.5 \\
5.5 \\
--\end{array}$ & $\begin{array}{l}-0 \\
-- \\
4.0 \\
4.0 \\
--\end{array}$ \\
\hline & $\begin{array}{l}72-03-16 \\
72-07-18 \\
72-10-25 \\
73-02-01 \\
73-04-24\end{array}$ & $\begin{array}{l}= \\
=- \\
= \\
=\end{array}$ & $\begin{array}{r}200 \\
109 \\
130 \\
49 \\
156\end{array}$ & $\begin{array}{l}8.5 \\
7.4 \\
5.0 \\
7.0 \\
4.5\end{array}$ & $\begin{array}{r}-- \\
=- \\
6.0 \\
--\end{array}$ & $\begin{array}{l}= \\
=- \\
=\end{array}$ & $\begin{array}{c}48 \\
15^{--} \\
6.8 \\
10^{-8}\end{array}$ & $\begin{array}{r}24 \\
.5 \\
1.5 \\
.8 \\
1.0\end{array}$ & $\begin{array}{l}.5 \\
3.5 \\
3.8 \\
5.6\end{array}$ & $\begin{array}{r}.6 \\
1.8 \\
3.4 \\
.4 \\
.5\end{array}$ \\
\hline & $\begin{array}{l}73-08-09 \\
73-10-15 \\
73-12-19 \\
70-01-14 \\
70-02-04\end{array}$ & $\begin{array}{r}= \\
=- \\
23.0 \\
20.0\end{array}$ & $\begin{array}{r}100 \\
47 \\
97 \\
291 \\
385\end{array}$ & $\begin{array}{c}7.5 \\
7.0 \\
17 \\
9.4 \\
10\end{array}$ & $\begin{array}{r}-- \\
-- \\
8.3 \\
7.3\end{array}$ & $\begin{array}{l}=- \\
\because- \\
207 \\
187\end{array}$ & $\begin{array}{l}9.8 \\
5.8 \\
17 \\
70^{--}\end{array}$ & $\begin{array}{r}1.1 \\
.5 \\
2.2 \\
.-2 \\
4.2\end{array}$ & $\begin{array}{l}4.1 \\
4.5 \\
7.7 \\
--. \\
6.8\end{array}$ & $\begin{array}{l}2.5 \\
4.0 \\
2.8 \\
--5 \\
1.5\end{array}$ \\
\hline \multirow{6}{*}{280231082343601} & $\begin{array}{l}70-03-31 \\
70-04-23 \\
70-06-15 \\
70-06-16 \\
70-06-25\end{array}$ & $\begin{array}{r}23.0 \\
22.0 \\
23.5 \\
.- \\
2.4\end{array}$ & $\begin{array}{r}370 \\
338 \\
380 \\
-- \\
316\end{array}$ & $\begin{array}{l}11 \\
10 \\
11 \\
9.4\end{array}$ & $\begin{array}{r}-- \\
7.4 \\
7.4 \\
8.1\end{array}$ & $\begin{array}{l}=- \\
226 \\
-- \\
230\end{array}$ & $68^{--}$ & $\begin{array}{l}-- \\
4.1 \\
--\end{array}$ & $\begin{array}{l}- \\
0.2 \\
--\end{array}$ & $\begin{array}{l}-- \\
-9 \\
--\end{array}$ \\
\hline & $\begin{array}{l}70-07-14 \\
70-08-04 \\
70-09-17 \\
70-10-22 \\
70-12-03\end{array}$ & $\begin{array}{l}24.0 \\
24.5 \\
28.2 \\
24.5 \\
20.0\end{array}$ & $\begin{array}{l}359 \\
343 \\
380 \\
358 \\
381\end{array}$ & $\begin{array}{l}17 \\
11 \\
9.0 \\
8.0 \\
9.0\end{array}$ & $\begin{array}{l}-2 \\
8.0 \\
7.7 \\
7.4 \\
7.5\end{array}$ & $\begin{array}{l}232 \\
228 \\
272 \\
236\end{array}$ & $\begin{array}{l}- \\
= \\
= \\
=\end{array}$ & $\begin{array}{l}= \\
=- \\
=- \\
=\end{array}$ & $\begin{array}{l}= \\
= \\
= \\
=\end{array}$ & $\begin{array}{l}= \\
= \\
= \\
=\end{array}$ \\
\hline & $\begin{array}{l}71-01-14 \\
71-02-24 \\
71-03-31 \\
71-04-28 \\
71-06-15\end{array}$ & $\begin{array}{l}-\overline{-} \\
21.5 \\
22.5 \\
25.0 \\
23.5\end{array}$ & $\begin{array}{l}-- \\
380 \\
372 \\
378 \\
375\end{array}$ & $\begin{array}{r}9.0 \\
9.0 \\
8.0 \\
20^{--}\end{array}$ & $\begin{array}{l}-.- \\
7.4 \\
7.4 \\
-. \\
7.3\end{array}$ & $\begin{array}{l}-- \\
238 \\
230 \\
-- \\
232\end{array}$ & $\begin{array}{l}= \\
= \\
z \\
-\end{array}$ & $\begin{array}{l}= \\
=- \\
=\end{array}$ & $\begin{array}{l}= \\
=- \\
= \\
=\end{array}$ & $\begin{array}{l}= \\
=- \\
=-\end{array}$ \\
\hline & $\begin{array}{l}71-07-28 \\
71-09-21 \\
71-10-28 \\
71-11-22 \\
71-12-27\end{array}$ & $\begin{array}{r}24.0 \\
24.0 \\
24.0 \\
=- \\
--\end{array}$ & $\begin{array}{l}362 \\
352 \\
371 \\
382 \\
378\end{array}$ & $\begin{array}{l}9.0 \\
10 \\
11 \\
10 \\
9.4\end{array}$ & $\begin{array}{r}7.4 \\
-.0 \\
7.0 \\
-.\end{array}$ & $\begin{array}{l}244 \\
234 \\
240 \\
--\end{array}$ & $\begin{array}{l}= \\
= \\
=-\end{array}$ & $22^{--}$ & $\begin{array}{l}=- \\
=- \\
55 \\
--\end{array}$ & $\begin{array}{l}=- \\
=- \\
-4 \\
--\end{array}$ \\
\hline & $\begin{array}{l}72-03-16 \\
72-07-18 \\
72-10-25 \\
73-02-01 \\
73-04-24\end{array}$ & $\begin{array}{l}= \\
=- \\
= \\
=\end{array}$ & $\begin{array}{l}370 \\
380 \\
360 \\
370 \\
379\end{array}$ & $\begin{array}{c}9.4 \\
16 \\
8.5 \\
12 \\
6.3\end{array}$ & $\begin{array}{l}-- \\
-- \\
7.7 \\
--\end{array}$ & $\begin{array}{l}= \\
=- \\
= \\
=\end{array}$ & $\begin{array}{l}140^{--} \\
70 \\
67\end{array}$ & $\begin{array}{l}20 \\
3.9 \\
9.6 \\
4.2 \\
4.2\end{array}$ & $\begin{array}{l}.5 \\
-5 \\
5.9 \\
5.7 \\
7.4\end{array}$ & $\begin{array}{l}.6 \\
.6 \\
.9 \\
.8 \\
.9\end{array}$ \\
\hline & $\begin{array}{l}73-08-09 \\
73-10-15 \\
73-12-10 \\
73-12-19 \\
70-01-30\end{array}$ & $\begin{array}{c}= \\
= \\
= \\
19.0\end{array}$ & $\begin{array}{l}379 \\
376 \\
376 \\
170\end{array}$ & $\begin{array}{c}8.0 \\
10^{-.} \\
10^{--}\end{array}$ & $\begin{array}{l}=- \\
=- \\
-- \\
6.6\end{array}$ & $\begin{array}{l}= \\
=- \\
= \\
79\end{array}$ & $\begin{array}{l}68 \\
69 \\
20 \\
20\end{array}$ & $\begin{array}{l}4.3 \\
3.9 \\
4.9 \\
-.8\end{array}$ & $\begin{array}{l}0.2 \\
6.3 \\
0.6 \\
5.5\end{array}$ & $\begin{array}{l}.7 \\
.7 \\
.7 \\
.8 \\
.8\end{array}$ \\
\hline \multirow{4}{*}{280231082342602} & $\begin{array}{l}70-03-31 \\
70-04-20 \\
70-06-01 \\
70-07-15 \\
70-08-04\end{array}$ & $\begin{array}{l}19.0 \\
23.0 \\
23.5 \\
26.0 \\
27.0\end{array}$ & $\begin{array}{l}219 \\
200 \\
170 \\
212 \\
253\end{array}$ & $\begin{array}{l}15 \\
10 \\
14 \\
10 \\
13\end{array}$ & $\begin{array}{l}=- \\
=- \\
6.7\end{array}$ & $\begin{array}{l}= \\
=- \\
=- \\
134\end{array}$ & $\begin{array}{l}= \\
= \\
= \\
=\end{array}$ & $\begin{array}{l}-- \\
-- \\
--\end{array}$ & $\begin{array}{l}= \\
= \\
=\end{array}$ & $\begin{array}{l}= \\
=- \\
=\end{array}$ \\
\hline & $\begin{array}{l}70-09-18 \\
70-10-23 \\
70-12-04 \\
71-01-13 \\
71-02-26\end{array}$ & $\begin{array}{l}27.2 \\
23.0 \\
24.0 \\
23.0 \\
22.5\end{array}$ & $\begin{array}{l}226 \\
213 \\
226 \\
166 \\
176\end{array}$ & $\begin{array}{l}11 \\
9.0 \\
10 \\
80 \\
9.0\end{array}$ & $\begin{array}{l}6.7 \\
6.8 \\
6.8 \\
6.2 \\
6.4\end{array}$ & $\begin{array}{r}139 \\
132 \\
96 \\
94 \\
114\end{array}$ & $\begin{array}{l}37^{--} \\
-- \\
--\end{array}$ & $\begin{array}{l}-. \\
2.5 \\
-- \\
--\end{array}$ & $\begin{array}{l}-- \\
6.8 \\
-- \\
--\end{array}$ & $\begin{array}{l}\ddot{1.2} \\
\overline{-} \\
--\end{array}$ \\
\hline & $\begin{array}{l}71-03-30 \\
71-04-28 \\
71-06-14 \\
71-07-27 \\
71-09-22\end{array}$ & $\begin{array}{l}21.0 \\
23.0 \\
24.0 \\
24.0 \\
\ldots\end{array}$ & $\begin{array}{l}172 \\
216 \\
240 \\
185 \\
210\end{array}$ & $\begin{array}{r}9.0 \\
9.0 \\
9.0 \\
8.0 \\
12\end{array}$ & $\begin{array}{l}6.5 \\
-. \\
6.6 \\
6.3 \\
6.2\end{array}$ & $\begin{array}{l}110 \\
120 \\
148 \\
144\end{array}$ & $\begin{array}{l}-- \\
\ddot{--} \\
--\end{array}$ & $\begin{array}{l}-- \\
=- \\
=\end{array}$ & $\begin{array}{l}= \\
= \\
= \\
=\end{array}$ & $\begin{array}{l}= \\
\because= \\
=\end{array}$ \\
\hline & $\begin{array}{l}71-10-29 \\
71-12-28 \\
72-04-27 \\
70-01-30 \\
70-03-31\end{array}$ & $\begin{array}{r}=- \\
=- \\
17.5 \\
21.5\end{array}$ & $\begin{array}{r}210 \\
185 \\
185 \\
62 \\
110\end{array}$ & $\begin{array}{l}10 \\
7.6 \\
13 \\
45 \\
7.0\end{array}$ & $\begin{array}{c}6.2 \\
-.0 \\
6.0 \\
6.2 \\
-. .\end{array}$ & $\begin{array}{r}144 \\
-- \\
-7\end{array}$ & $\begin{array}{l}26^{--} \\
65^{-7} \\
3.7 \\
-\end{array}$ & $\begin{array}{r}3.7 \\
4.5 \\
.7 \\
--\end{array}$ & $\begin{array}{r}-. \\
5.6 \\
8.4 \\
3.6 \\
.-\end{array}$ & $\begin{array}{l}-. \\
1.6 \\
1.7 \\
0.0 \\
--\end{array}$ \\
\hline
\end{tabular}




\begin{tabular}{|c|c|c|c|c|c|c|c|c|c|c|}
\hline STATION NUMEER & $\begin{array}{c}\text { DATE } \\
\text { OF } \\
\text { SAMPLE }\end{array}$ & $\begin{array}{l}\text { TEMPER- } \\
\text { ATUREE } \\
\text { (UEG C) }\end{array}$ & $\begin{array}{l}\text { SPE- } \\
\text { CIFIC } \\
\text { CON- } \\
\text { DUCT- } \\
\text { ANCE } \\
\text { (MICRO- } \\
\text { MHOS) }\end{array}$ & $\begin{array}{l}\text { CHLO- } \\
\text { RIDE, } \\
\text { DIS- } \\
\text { SOLVED } \\
\text { (MG/L } \\
\text { AS CL) }\end{array}$ & $\begin{array}{c}\text { PH } \\
\text { (UNITS) }\end{array}$ & $\begin{array}{l}\text { ALKA- } \\
\text { LINITY } \\
\text { (MG/L } \\
\text { AS } \\
\text { CACO3) }\end{array}$ & $\begin{array}{l}\text { CALCIUM } \\
\text { DIS- } \\
\text { SOLVED } \\
\text { (MG L } \\
\text { AS CA) }\end{array}$ & $\begin{array}{l}\text { MAGNE- } \\
\text { SIUM, } \\
\text { OIS- } \\
\text { SOLVEU } \\
\text { (MG L } \\
\text { AS MG) }\end{array}$ & $\begin{array}{l}\text { SOOIUM, } \\
\text { OIS- } \\
\text { SOLVED } \\
\text { (MGAL } \\
\text { AS NA) }\end{array}$ & $\begin{array}{l}\text { POTAS- } \\
\text { SIUM, } \\
\text { DIS- } \\
\text { SOLVED } \\
\text { (MG/L } \\
\text { AS K) }\end{array}$ \\
\hline 280231082342001 & $\begin{array}{l}70-04-20 \\
71-02-26\end{array}$ & $\begin{array}{l}24.5 \\
23.0\end{array}$ & $\begin{array}{l}103 \\
238\end{array}$ & 8.0 & $\overline{0}$ & 270 & $=$ & $=$ & $=$ & $=$ \\
\hline \multirow[t]{2}{*}{280233082340702} & $\begin{array}{l}73-02-01 \\
73-08-13\end{array}$ & $=$ & $\begin{array}{l}420 \\
390 \\
302\end{array}$ & 120 & 7.7 & $=$ & $\begin{array}{l}82 \\
70 \\
72\end{array}$ & $\begin{array}{l}6.5 \\
5.4\end{array}$ & $\begin{array}{l}6.8 \\
6.8\end{array}$ & $\begin{array}{l}1.0 \\
.9\end{array}$ \\
\hline & $73-10-16$ & $\cdots$ & 392 & 9.0 & - & - & 72 & $4 \cdot 2$ & 6.4 & $\cdot 7$ \\
\hline \multirow[t]{4}{*}{280233082340701} & $\begin{array}{l}73-12-19 \\
72-01-27\end{array}$ & $=$ & $\begin{array}{l}388 \\
182\end{array}$ & $\begin{array}{l}7.4 \\
9.0\end{array}$ & $\overrightarrow{7.1}$ & 75 & ${ }^{68} \ldots$ & 5.3 & $7: 0$ & 1.7 \\
\hline & $72-07-17$ & -- & 360 & 20 & -- & -- & 110 & $<.2$ & - & 4.2 \\
\hline & $73-04-25$ & - & 155 & 23 & -- & $=$ & 10 & $<.2$ & 12 & 1.0 \\
\hline & $73-08-13$ & - & 150 & 22 & -- & $\cdots$ & 9.4 & 2.8 & 11 & .6 \\
\hline \multirow{19}{*}{280236082343902} & $\begin{array}{l}73-10-16 \\
73-12-20\end{array}$ & $=$ & $\begin{array}{l}132 \\
138\end{array}$ & $\begin{array}{l}20 \\
23\end{array}$ & $=$ & $=$ & 6.6 & $<.2$ & 11 & .5 \\
\hline & $70-01-19$ & 23.0 & - & 17 & - & 200 & & $\ldots$ & -. & $\because$ \\
\hline & $\begin{array}{l}70-02-04 \\
70-02-24\end{array}$ & 22.0 & $\begin{array}{l}384 \\
404\end{array}$ & 15 & 7.3 & 190 & $62 \ldots$ & 4.6 & 8.5 & .8 \\
\hline & $70-03-31$ & 24.0 & 371 & 17 & -- & -- & $-\infty$ & $-\infty$ & $\cdots$ & $\cdots$ \\
\hline & $70-04-23$ & 25.0 & 353 & 20 & $\cdots$ & -- & $\cdots$ & -- & $\cdots$ & - \\
\hline & $70-06-25$ & 26.0 & 334 & 17 & 8.1 & 230 & $=$ & -- & -- & $=$ \\
\hline & $70-08-04$ & $\begin{array}{l}26.5 \\
24.5\end{array}$ & $\begin{array}{l}389 \\
405\end{array}$ & $\begin{array}{l}17 \\
17\end{array}$ & $7 . \overline{2}$ & 233 & $\because$ & 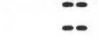 & $=$ & 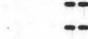 \\
\hline & $70-0 y-17$ & 27.0 & 402 & 10 & 7.6 & 232 & -. & -- & -- & - \\
\hline & $70-10-22$ & 25.0 & 372 & 16 & 7.3 & 230 & 69 & 4.5 & 9.3 & .9 \\
\hline & $\begin{array}{l}70-12-02 \\
71-01-14\end{array}$ & 23.5 & 410 & 16 & $7 \cdot 5$ & 240 & $=$ & $=$ & $=$ & $=-$ \\
\hline & $\begin{array}{l}71-0.1-14 \\
71-02-24\end{array}$ & $\begin{array}{l}23.0 \\
23.0\end{array}$ & $\begin{array}{l}394 \\
404\end{array}$ & $16^{--}$ & $\begin{array}{l}7.5 \\
7.5\end{array}$ & $\begin{array}{l}226 \\
234\end{array}$ & $=$ & $\overline{-}$ & $\overline{-}$ & $=$ \\
\hline & $71-03-31$ & 22.0 & 390 & 15 & 7.5 & 230 & - & -- & $\ldots$ & - \\
\hline & $71-04-28$ & 26.0 & 386 & 15 & $\cdots$ & - & -- & - & -- & $\cdots$ \\
\hline & $71-06-14$ & 24.5 & 400 & 15 & 7.4 & 228 & $\cdots$ & -- & $\cdots$ & $\cdots$ \\
\hline & $71-07-28$ & 26.0 & 395 & 16 & 7.2 & 244 & - & - & - & - \\
\hline & $72-03-16$ & -- & 390 & 15 & -- & -- & 63 & -- & - & -- \\
\hline & $72-07-18$ & -- & 368 & - & -- & $\cdots$ & -- & 12 & -- & .3 \\
\hline & $72-10-25$ & -- & 347 & - & -- & - & 60 & 4.3 & 7.0 & .9 \\
\hline & $73-04-23$ & 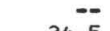 & 390 & 13 & -- & 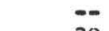 & 66 & 4.8 & 9.0 & $\cdot 9$ \\
\hline \multirow[t]{7}{*}{280233082342301} & $70-08-05$ & 24.5 & 64 & 3.0 & 9.0 & 39 & - & $\cdots$ & -- & $\overline{-}$ \\
\hline & & & & & & & & & & \\
\hline & $71-01-13$ & 22.0 & 83 & $\begin{array}{l}6.0 \\
4.0\end{array}$ & $\begin{array}{l}9.4 \\
9.1\end{array}$ & $\begin{array}{l}96 \\
40\end{array}$ & $\therefore$ & $=$ & $\ldots$ & $=$ \\
\hline & $71-02-24$ & 22.0 & 138 & 7.0 & 9.1 & 62 & - & -- & - & - \\
\hline & $71-03-31$ & 24.0 & 208 & 9.0 & 8.2 & 100 & -- & -- & $\cdots$ & -- \\
\hline & $71-04-28$ & 23.0 & 216 & 8.0 & -- & $\cdots$ & -- & -- & - & - \\
\hline & $\begin{array}{l}71-06-14 \\
71-07-27\end{array}$ & $\begin{array}{l}23.0 \\
24.0\end{array}$ & $\begin{array}{l}184 \\
168\end{array}$ & $10^{8.0}$ & $\begin{array}{l}8.5 \\
7.2\end{array}$ & $\begin{array}{l}92 \\
80\end{array}$ & $=-$ & $=$ & $=$ & $=$ \\
\hline \multirow[t]{10}{*}{280233082342302} & $70-02-05$ & 21.5 & 58 & 5.5 & 7.0 & 15 & 7.0 & 1.8 & 7.1 & .5 \\
\hline & $\begin{array}{l}70-08-05 \\
\end{array}$ & 23.5 & 69 & 5.0 & 6.0 & 49 & - & - & $\cdots$ & - \\
\hline & $70-09-18$ & 28.0 & 93 & 5.0 & 6.6 & 60 & -- & $\cdots$ & - & $\cdots$ \\
\hline & $70-10-26$ & 26.5 & 73 & 4.0 & 6.5 & 54 & $\cdots$ & -- & $\cdots$ & - \\
\hline & $70-12-03$ & 22.5 & 97 & 4.0 & 6.2 & 40 & $=$ & $=$ & $=$ & $=-$ \\
\hline & $\begin{array}{l}1-01-13 \\
71-02-24\end{array}$ & $\begin{array}{l}22.0 \\
21.0\end{array}$ & $\begin{array}{l}45 \\
45\end{array}$ & $\begin{array}{l}3.0 \\
7.0\end{array}$ & $\begin{array}{l}5.9 \\
6.1\end{array}$ & $\begin{array}{l}24 \\
38\end{array}$ & $=$ & $=$ & $=$ & $=$ \\
\hline & $71-03-31$ & 21.0 & 36 & 4.0 & 6.7 & 44 & - & -- & $\cdots$ & $\cdots$ \\
\hline & $71-04-28$ & 21.5 & 38 & 4.0 & $=$ & $=$ & -- & -- & $\cdots$ & $\because$ \\
\hline & $71-06-14$ & 23.0 & 35 & 6.0 & 6.7 & E78 & $\cdots$ & - & $\because$ & - \\
\hline & $70-01-30$ & 25.0 & $\begin{array}{l}57 \\
64\end{array}$ & 5.0 & $\begin{array}{l}6.0 \\
6.4\end{array}$ & $\begin{array}{l}56 \\
17\end{array}$ & $\ldots$ & $=$ & $\ldots$ & $=$ \\
\hline \multirow{8}{*}{$280<3308<34<603$} & $70-03-31$ & 22.0 & 74 & 7.0 & $\ldots$ & $\because$ & $\cdots$ & -- & -- & $\cdots$ \\
\hline & $70-04-23$ & $\cdots$ & 104 & 8.0 & -- & - & - & -- & 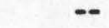 & -- \\
\hline & $70-06-01$ & 25.5 & 69 & 8.0 & - & -- & $=$ & $=$ & $=$ & $=$ \\
\hline & $70-07-15$ & 27.0 & 340 & 10 & -- & $\cdots$ & $\cdots$ & $=$ & $=$ & $=$ \\
\hline & $\begin{array}{l}70-09-18 \\
71-04-28\end{array}$ & $22 . \overline{5}$ & $\begin{array}{l}372 \\
362\end{array}$ & $\begin{array}{l}12 \\
9.0\end{array}$ & $=$ & $=$ & $=$ & $=$ & $=$ & $=$ \\
\hline & $71-09-22$ & $\cdots$ & 94 & 4.0 & 6.5 & 38 & -- & -- & $\cdots$ & - \\
\hline & $71-10-29$ & -- & 76 & 7.0 & 5.9 & 20 & - & -- & $\cdots$ & $\cdots$ \\
\hline & $71-12-28$ & $=$ & 23 & 8.0 & 6.4 & 27 & 8.8 & 130 & 4.1 & .4 \\
\hline \multirow[t]{13}{*}{280233082342602} & $70-01-30$ & 20.0 & 102 & 5.5 & 6.4 & 58 & 7.8 & 1.7 & 4.1 & .6 \\
\hline & $70-03-31$ & 21.0 & & 8.0 & -- & - & - & -- & $\cdots$ & $\cdots$ \\
\hline & $70-04-23$ & 22.0 & 112 & 10 & $\because$ & $-\infty$ & $\because$ & $\because$ & $=$ & $=$ \\
\hline & $\begin{array}{l}70-06-01 \\
70-07-15\end{array}$ & $\begin{array}{l}24.0 \\
26.0\end{array}$ & $\begin{array}{l}41 \\
55\end{array}$ & 5.0 & $=$ & $\because$ & $=$ & $=$ & $\ldots$ & $=$ \\
\hline & $70-08-04$ & 26.0 & 79 & 7.0 & 6.6 & 41 & - & -- & -- & $\cdots$ \\
\hline & $70-09-18$ & 27.0 & 62 & 6.0 & 6.4 & 42 & $=$ & $-\overline{7}$ & 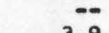 & 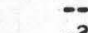 \\
\hline & $\begin{array}{l}70-10-23 \\
70-12-04\end{array}$ & $\begin{array}{l}25.0 \\
24.0\end{array}$ & $\begin{array}{l}44 \\
78\end{array}$ & $\begin{array}{l}6.0 \\
8.0\end{array}$ & $\begin{array}{l}6.3 \\
6.1\end{array}$ & $\begin{array}{l}40 \\
22\end{array}$ & 5.0 & $\because 7$ & $\begin{array}{l}3.9 \\
-.-\end{array}$ & $\because 2$ \\
\hline & $71-01-13$ & 22.0 & 36 & - & 5.4 & 12 & 2.0 & .5 & 3.6 & .1 \\
\hline & $71-02-26$ & 23.0 & 52 & 5.0 & 6.0 & 2 & -9 & $-n_{-1}$ & & \\
\hline & $71-03-30$ & 21.0 & 6 & $=$ & 6.3 & -- & - & -- & $\cdots$ & - \\
\hline & $\begin{array}{l}1-04-28 \\
71-06-14\end{array}$ & $\begin{array}{l}22.0 \\
23.5\end{array}$ & 36 & $\begin{array}{l}5.0 \\
5.0\end{array}$ & $6 . \overline{2}$ & 30 & $=$ & $=$ & $=$ & $\ldots$ \\
\hline & $\begin{array}{l}71-07-28 \\
71-00-? 2\end{array}$ & 24.5 & 38 & 5.0 & 6.5 & 36 & $=$ & $=$ & $\because$ & $=$ \\
\hline & & & & 6.0 & 6.3 & & 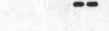 & $\cdots$ & $\cdots$ & $-\infty$ \\
\hline
\end{tabular}




\begin{tabular}{|c|c|c|c|c|c|c|c|c|c|c|}
\hline STATION NUMBER & $\begin{array}{c}\text { DATE } \\
\text { OF } \\
\text { SAMPLE }\end{array}$ & $\begin{array}{l}\text { TEMPER- } \\
\text { ATURE } \\
\text { (DEG C) }\end{array}$ & $\begin{array}{l}\text { SPE- } \\
\text { CIFIC } \\
\text { CON- } \\
\text { OUCT- } \\
\text { ANCE } \\
\text { (MICRO- } \\
\text { MHOS) }\end{array}$ & $\begin{array}{l}\text { CHLO- } \\
\text { KIOE, } \\
\text { DIS- } \\
\text { SOLVED } \\
\text { (MG/L } \\
\text { AS CL) }\end{array}$ & $\begin{array}{c}\text { PH } \\
\text { (UNITS) }\end{array}$ & $\begin{array}{l}\text { ALKA- } \\
\text { LINITY } \\
\text { (MG/L } \\
\text { AS } \\
\text { CACO3) }\end{array}$ & $\begin{array}{l}\text { CALCIUM } \\
\text { OIS- } \\
\text { SOLVEO } \\
\text { (MG/L } \\
\text { AS CA) }\end{array}$ & $\begin{array}{l}\text { MAGNE- } \\
\text { SIUM, } \\
\text { OIS- } \\
\text { SOLVEO } \\
\text { (MG/L } \\
\text { AS MG) }\end{array}$ & $\begin{array}{l}\text { SOUIUM, } \\
\text { DIS- } \\
\text { SOLVED } \\
\text { (MG/L } \\
\text { AS NA) }\end{array}$ & $\begin{array}{l}\text { POTAS- } \\
\text { SIUM, } \\
\text { DIS- } \\
\text { SOLVEU } \\
\text { (MG/L } \\
\text { AS K) }\end{array}$ \\
\hline 280233082342602 & $\begin{array}{l}71-10-29 \\
71-11-22 \\
71-12-28 \\
72-07-18 \\
72-10-20\end{array}$ & 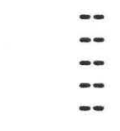 & $\begin{array}{r}75 \\
69 \\
90 \\
405 \\
90\end{array}$ & $\begin{array}{l}9.0 \\
8.4 \\
10 \\
15 \\
12\end{array}$ & $\begin{array}{r}6.1 \\
-.3 \\
-- \\
--\end{array}$ & $\begin{array}{l}8 \\
-- \\
22 \\
--\end{array}$ & $\begin{array}{r}\ddot{-} \\
5.0 \\
\ddot{0-} \\
5.8\end{array}$ & $\begin{array}{l}-. \\
1.1 \\
-- \\
3.6 \\
1.6\end{array}$ & $\begin{array}{r}3.0 \\
\overline{--} \\
5.4\end{array}$ & $\begin{array}{r}-. \\
-1 \\
1.9 \\
-.\end{array}$ \\
\hline 280233082342601 & $\begin{array}{l}70-01-30 \\
70-03-30 \\
70-04-23 \\
70-06-01 \\
70-06-25\end{array}$ & $\begin{array}{l}20.0 \\
20.0 \\
23.0 \\
25.0 \\
26.0\end{array}$ & $\begin{array}{r}71 \\
24 \\
358 \\
280 \\
274\end{array}$ & $\begin{array}{l}7.0 \\
12 \\
10 \\
12 \\
10\end{array}$ & $\begin{array}{c}6.4 \\
=- \\
=- \\
8.0\end{array}$ & $\begin{array}{l}20 \\
=- \\
190\end{array}$ & $\begin{array}{l}= \\
=- \\
= \\
=\end{array}$ & $\begin{array}{l}= \\
=- \\
=-\end{array}$ & $\begin{array}{l}=- \\
=- \\
--\end{array}$ & $\begin{array}{l}= \\
=- \\
=-\end{array}$ \\
\hline & $\begin{array}{l}70-07-15 \\
70-08-04 \\
70-09-18 \\
70-10-26 \\
70-12-04\end{array}$ & $\begin{array}{l}27.0 \\
27.0 \\
28.0 \\
26.0 \\
22.5\end{array}$ & $\begin{array}{l}326 \\
350 \\
342 \\
342 \\
341\end{array}$ & $\begin{array}{l}10 \\
9.0 \\
12 \\
10 \\
11\end{array}$ & $\begin{array}{l}7 .- \\
7.3 \\
7.5 \\
7.4 \\
7.4\end{array}$ & $\begin{array}{l}205 \\
235 \\
212 \\
204\end{array}$ & $\begin{array}{l}= \\
=- \\
=\end{array}$ & $\begin{array}{l}= \\
=- \\
=-\end{array}$ & $\begin{array}{l}-- \\
= \\
= \\
--\end{array}$ & $\begin{array}{l}= \\
=- \\
=\end{array}$ \\
\hline & $\begin{array}{l}71-01-13 \\
71-02-26 \\
71-03-30 \\
71-06-14 \\
71-07-28\end{array}$ & $\begin{array}{l}22.5 \\
23.0 \\
21.0 \\
24.5 \\
25.0\end{array}$ & $\begin{array}{l}322 \\
354 \\
360 \\
378 \\
372\end{array}$ & $\begin{array}{l}9.0 \\
9.0 \\
9.0 \\
10 \\
10\end{array}$ & $\begin{array}{l}7.5 \\
7.5 \\
7.7 \\
7.5 \\
7.5\end{array}$ & $\begin{array}{l}200 \\
226 \\
232 \\
228 \\
292\end{array}$ & $\begin{array}{l}56 \\
=- \\
=- \\
=-\end{array}$ & $\begin{array}{c}3.8 \\
=- \\
=- \\
=-\end{array}$ & $\begin{array}{c}6.6 \\
=- \\
=- \\
=-\end{array}$ & $\begin{array}{l}1.4 \\
=- \\
=- \\
--\end{array}$ \\
\hline & $\begin{array}{l}71-09-22 \\
71-10-29 \\
71-11-22 \\
71-12-28 \\
73-04-27\end{array}$ & $\begin{array}{r}28.0 \\
=- \\
=- \\
=-\end{array}$ & $\begin{array}{l}360 \\
340 \\
362 \\
365 \\
185\end{array}$ & $\begin{array}{c}9.0 \\
10^{\circ} \\
9.0 \\
10^{\circ} \\
7.2\end{array}$ & $\begin{array}{l}7.5 \\
7.4 \\
-- \\
7.4 \\
--\end{array}$ & $\begin{array}{l}228 \\
224 \\
224 \\
-8 \\
248\end{array}$ & $65^{--}$ & $\begin{array}{l}-. \\
4.4 \\
-. \\
2.1\end{array}$ & $\begin{array}{l}=- \\
5.0 \\
0 \\
6.3\end{array}$ & $\begin{array}{l}-- \\
-. \\
-5 \\
.5\end{array}$ \\
\hline $\begin{array}{l}280235082335801 \\
280235082341701\end{array}$ & $\begin{array}{l}73-02-01 \\
73-04-26 \\
71-01-13 \\
71-10-29 \\
71-12-27\end{array}$ & $\begin{array}{l}= \\
= \\
= \\
=\end{array}$ & $\begin{array}{r}380 \\
385 \\
83 \\
290 \\
212\end{array}$ & $\begin{array}{c}10 \\
8.0 \\
4.4 \\
10 \\
8.8\end{array}$ & $\begin{array}{l}7.5 \\
7.4 \\
7.0 \\
7.2 \\
-.\end{array}$ & $\begin{array}{r}-- \\
194 \\
168 \\
136 \\
--\end{array}$ & $\begin{array}{l}72 \\
70 \\
34^{--}\end{array}$ & $\begin{array}{r}3.8 \\
4.0 \\
210^{--}\end{array}$ & $\begin{array}{r}6.3 \\
7.8 \\
-. \\
-- \\
5.8\end{array}$ & $\begin{array}{l}.5 \\
.8 \\
-. \\
-. \\
.9\end{array}$ \\
\hline 280236082340701 & $\begin{array}{l}72-01-27 \\
72-03-16 \\
72-07-19 \\
72-10-26 \\
73-02-01\end{array}$ & $\begin{array}{l}= \\
= \\
=\end{array}$ & $\begin{array}{l}182 \\
210 \\
266 \\
185 \\
182\end{array}$ & $\begin{array}{l}9.0 \\
12 \\
13 \\
6.6 \\
28\end{array}$ & $\begin{array}{r}7.1 \\
=- \\
=- \\
7.0\end{array}$ & $\begin{array}{l}75 \\
=- \\
=- \\
=-\end{array}$ & $\begin{array}{l}30^{--} \\
57 \\
28 \\
14\end{array}$ & $\begin{array}{r}-\overline{4} \\
12.4 \\
1.9 \\
3.7\end{array}$ & $\begin{array}{r}-\overline{1.0} \\
5.4 \\
14\end{array}$ & $\begin{array}{l}.- \\
.1 \\
.1 \\
.4 \\
.7\end{array}$ \\
\hline 280235082341702 & $\begin{array}{l}73-08-01 \\
73-08-10 \\
73-10-17 \\
73-12-19 \\
72-07-19\end{array}$ & $\begin{array}{l}\square \\
\therefore \\
=\end{array}$ & $\begin{array}{l}385 \\
138 \\
219 \\
227 \\
-.\end{array}$ & $\begin{array}{l}10 \\
7.0 \\
44 \\
48 \\
12\end{array}$ & $\begin{array}{l}= \\
=- \\
=-\end{array}$ & $\begin{array}{l}= \\
= \\
=\end{array}$ & $\begin{array}{l}66 \\
19 \\
16 \\
13 \\
31\end{array}$ & $\begin{array}{l}2.5 \\
1.0 \\
3.4 \\
4.0 \\
4.0\end{array}$ & $\begin{array}{l}5.3 \\
4.4 \\
18 \\
21 \\
-.\end{array}$ & $\begin{array}{l}.3 \\
.0 \\
.0 \\
.2 \\
.9\end{array}$ \\
\hline 280236082342601 & $\begin{array}{l}71-09-22 \\
71-10-28 \\
72-01-27 \\
72-03-16 \\
72-07-18\end{array}$ & 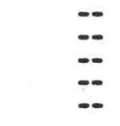 & $\begin{array}{l}494 \\
308 \\
210 \\
140 \\
--\end{array}$ & $\begin{array}{l}11 \\
13 \\
7.0 \\
12 \\
14\end{array}$ & $\begin{array}{l}7.8 \\
6.8 \\
7.1 \\
-- \\
--\end{array}$ & $\begin{array}{r}748 \\
182 \\
162 \\
-- \\
--\end{array}$ & 20 & $\begin{array}{l}= \\
=- \\
5.0 \\
--\end{array}$ & $\begin{array}{l}= \\
\because- \\
\because\end{array}$ & $\begin{array}{l}-- \\
-- \\
-1 \\
.4\end{array}$ \\
\hline & $\begin{array}{l}72-10-26 \\
73-04-27 \\
73-08-09 \\
73-10-16 \\
73-12-20\end{array}$ & $\begin{array}{l}= \\
= \\
= \\
=\end{array}$ & $\begin{array}{l}230 \\
158 \\
198 \\
61 \\
158\end{array}$ & $\begin{array}{l}11 \\
17 \\
22 \\
25 \\
25\end{array}$ & $\begin{array}{l}= \\
=- \\
=-\end{array}$ & $\begin{array}{l}= \\
= \\
=\end{array}$ & $\begin{array}{l}41 \\
20 \\
25 \\
22 \\
19\end{array}$ & $\begin{array}{l}1.9 \\
1.7 \\
2.5 \\
2.4 \\
3.3\end{array}$ & $\begin{array}{l}4.1 \\
8.1 \\
9.4 \\
10 \\
10\end{array}$ & $\begin{array}{l}.2 \\
.3 \\
.1 \\
.0 \\
.4\end{array}$ \\
\hline $\begin{array}{l}280237082342701 \\
280237082342901 \\
280237082343801\end{array}$ & $\begin{array}{l}73-04-27 \\
72-07-18 \\
70-02-04 \\
70-10-22 \\
72-07-18\end{array}$ & $\begin{array}{r}=- \\
21.0 \\
25.0 \\
--\end{array}$ & $\begin{array}{r}1630 \\
5700 \\
388 \\
364 \\
352\end{array}$ & $\begin{array}{r}69 \\
220 \\
13 \\
15 \\
17\end{array}$ & $\begin{array}{r}-- \\
-- \\
8.3 \\
8.2 \\
-.\end{array}$ & $\begin{array}{r}-0 \\
159 \\
177 \\
--\end{array}$ & $\begin{array}{r}220 \\
150 \\
59 \\
65 \\
47\end{array}$ & $\begin{array}{l}23 \\
110 \\
3.7 \\
4.4 \\
2.4\end{array}$ & $\begin{array}{r}78 \\
7.0 \\
7.6 \\
--\end{array}$ & $\begin{array}{r}26 \\
181 \\
1.8 \\
1.1 \\
1.1\end{array}$ \\
\hline 280237082343802 & $\begin{array}{l}72-10-25 \\
73-02-01 \\
73-04-24 \\
70-01-20 \\
70-02-04\end{array}$ & $\begin{array}{r}=- \\
-0 \\
22.0 \\
20.0\end{array}$ & $\begin{array}{l}275 \\
282 \\
357 \\
305 \\
334\end{array}$ & $\begin{array}{c}15 \\
16 \\
13 \\
12 \\
6.0\end{array}$ & $\begin{array}{l}7.7 \\
7.6 \\
8.2 \\
7.3\end{array}$ & $\begin{array}{l}-0 \\
164 \\
200 \\
171\end{array}$ & $\begin{array}{l}41 \\
50 \\
60 \\
61\end{array}$ & $\begin{array}{l}3.4 \\
3.6 \\
4.5 \\
-.8 \\
1.8\end{array}$ & $\begin{array}{l}6.0 \\
6.1 \\
8.1 \\
-.5 \\
3.4\end{array}$ & $\begin{array}{r}1.0 \\
.7 \\
1.3 \\
-.9 \\
.9\end{array}$ \\
\hline 280237082343803 & $\begin{array}{l}70-10-22 \\
70-10-27 \\
73-02-01 \\
73-04-24 \\
70-01-20\end{array}$ & $\begin{array}{r}.4 \\
24.0 \\
-0 \\
-0 \\
22.0\end{array}$ & $\begin{array}{r}-2 \\
172 \\
105 \\
105 \\
58\end{array}$ & $\begin{array}{l}12-- \\
6.0 \\
8.0 \\
9.0\end{array}$ & $\begin{array}{l}-. \\
7.7 \\
7.5 \\
6.5 \\
6.1\end{array}$ & $\begin{array}{r}131 \\
38 \\
16\end{array}$ & $\begin{array}{l}-- \\
17^{--} \\
15\end{array}$ & $\begin{array}{r}-. \\
-.8 \\
1.0 \\
--\end{array}$ & $\begin{array}{l}=- \\
1.9 \\
2.3 \\
-.\end{array}$ & $\begin{array}{l}-- \\
-8 \\
.6 \\
--\end{array}$ \\
\hline 280238082340902 & $\begin{array}{l}70-02-04 \\
72-07-18 \\
73-08-01 \\
73-08-10 \\
73-10-17\end{array}$ & $\begin{array}{c}20.0 \\
=- \\
=- \\
=\end{array}$ & $\begin{array}{r}91 \\
202 \\
400 \\
318 \\
307\end{array}$ & $\begin{array}{l}4.5 \\
4.6 \\
11 \\
12 \\
11\end{array}$ & $\begin{array}{c}6.6 \\
=- \\
=- \\
=-\end{array}$ & $\begin{array}{l}30 \\
=- \\
=- \\
=-\end{array}$ & $\begin{array}{l}11 \\
80^{--} \\
49 \\
54\end{array}$ & $\begin{array}{l}1.0 \\
--. \\
4.2 \\
2.4 \\
1.5\end{array}$ & $\begin{array}{l}3.0 \\
.- \\
8.6 \\
8.0 \\
8.9\end{array}$ & $\begin{array}{l}.9 \\
.5 \\
.6 \\
.3 \\
.3\end{array}$ \\
\hline 280238082340901 & $\begin{array}{l}73-12-19 \\
73-08-01 \\
73-08-10 \\
73-10-17 \\
73-12-19\end{array}$ & 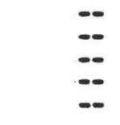 & $\begin{array}{l}270 \\
430 \\
390 \\
386 \\
360\end{array}$ & $\begin{array}{r}10 \\
8.0 \\
8.0 \\
9.0 \\
8.4\end{array}$ & $\begin{array}{l}= \\
=- \\
=-\end{array}$ & $\begin{array}{l}= \\
= \\
= \\
=\end{array}$ & $\begin{array}{l}45 \\
68 \\
70 \\
70 \\
49\end{array}$ & $\begin{array}{l}3.0 \\
5.5 \\
4.6 \\
4.2 \\
5.4\end{array}$ & $\begin{array}{r}9.0 \\
11 \\
7.0 \\
6.0 \\
7.7\end{array}$ & $\begin{array}{l}1.6 \\
.6 \\
1.3 \\
1.3 \\
1.1\end{array}$ \\
\hline 280238082343202 & $\begin{array}{l}70-02-04 \\
70-10-22 \\
70-12-02 \\
71-02-24 \\
71-09-21\end{array}$ & $\begin{array}{r}=- \\
=-0 \\
25.0 \\
23.0 \\
28.0\end{array}$ & $\begin{array}{l}278 \\
172 \\
226 \\
404 \\
218\end{array}$ & $\begin{array}{l}5.0 \\
3.0 \\
4.0 \\
5.0 \\
7.0\end{array}$ & $\begin{array}{l}7.0 \\
7.9 \\
7.4 \\
7.8 \\
8.0\end{array}$ & $\begin{array}{r}136 \\
102 \\
110 \\
88 \\
152\end{array}$ & $\begin{array}{r}49 \\
=- \\
=- \\
=-\end{array}$ & $\begin{array}{r}1.0 \\
\because- \\
= \\
=\end{array}$ & $\begin{array}{r}2.8 \\
\because- \\
=- \\
=-\end{array}$ & $\begin{array}{l}86 \\
=- \\
=-\end{array}$ \\
\hline
\end{tabular}


TABLE 6A,--WATER-QUALITY DATA FOR GROUND-WATER SITES AT THE ROCKY CREEK LANDFILL--CONTINUED

\begin{tabular}{|c|c|c|c|c|c|c|c|c|c|c|}
\hline STATION NUMBER & $\begin{array}{c}\text { DAIE } \\
\text { OF } \\
\text { SAMPLE }\end{array}$ & $\begin{array}{l}\text { TEMPER- } \\
\text { ATURE } \\
\text { (DEG C) }\end{array}$ & $\begin{array}{l}\text { SPE- } \\
\text { CIFIC } \\
\text { CON- } \\
\text { OUCT- } \\
\text { ANCE } \\
\text { (MICRO- } \\
\text { MHOS) }\end{array}$ & $\begin{array}{l}\text { CHLO- } \\
\text { KIDE, } \\
\text { OIS- } \\
\text { SOLVED } \\
\text { (MG/L } \\
\text { AS CL) }\end{array}$ & $\begin{array}{c}\text { PH } \\
\text { (UNITS) }\end{array}$ & $\begin{array}{l}\text { ALKA- } \\
\text { LINITY } \\
\text { (MG/L } \\
\text { AS } \\
\text { CACU3) }\end{array}$ & $\begin{array}{l}\text { CALCIUM } \\
\text { DIS- } \\
\text { SOLVED } \\
\text { (MG/L } \\
\text { AS CA) }\end{array}$ & $\begin{array}{l}\text { MAGNE- } \\
\text { SIUM, } \\
\text { DIS- } \\
\text { SOLVEO } \\
\text { (MG/L } \\
\text { AS MG) }\end{array}$ & $\begin{array}{l}\text { SOOIUM, } \\
\text { OIS- } \\
\text { SOLVED } \\
\text { (MG/L } \\
\text { AS NA) }\end{array}$ & $\begin{array}{l}\text { POTAS- } \\
\text { SIUM, } \\
\text { DIS- } \\
\text { SOLVED } \\
\text { (MG/L } \\
\text { AS K) }\end{array}$ \\
\hline 280238082343202 & $\begin{array}{l}71-11-22 \\
71-12-27 \\
72-01-26 \\
72-03-16 \\
72-06-22\end{array}$ & $\begin{array}{l}= \\
= \\
=\end{array}$ & $\begin{array}{r}515 \\
252 \\
106 \\
250 \\
90\end{array}$ & $\begin{array}{l}4.7 \\
9.0 \\
7.0 \\
7.4 \\
2.6\end{array}$ & $\begin{array}{l}7.5 \\
=- \\
\overline{--}\end{array}$ & 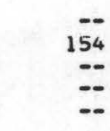 & $\begin{array}{c}29 \\
110^{-0} \\
12\end{array}$ & $\begin{array}{l}1.3 \\
-.- \\
4.6 \\
1.1\end{array}$ & \begin{tabular}{r}
2.0 \\
\hdashline-0 \\
.0 \\
3.0
\end{tabular} & $\begin{array}{r}.4 \\
-. \\
.8 \\
1.8\end{array}$ \\
\hline \multirow[t]{5}{*}{280238082343201} & $\begin{array}{l}72-07-18 \\
72-07-21 \\
70-01-07 \\
70-02-02 \\
70-02-04\end{array}$ & $\begin{array}{r}=- \\
8.0 \\
18.5 \\
21.0\end{array}$ & $\begin{array}{r}180 \\
106 \\
72 \\
45 \\
378\end{array}$ & $\begin{array}{r}8.2 \\
12 \\
4.8 \\
9.0\end{array}$ & $\begin{array}{l}-- \\
0.3 \\
6.3 \\
7.9\end{array}$ & $\begin{array}{r}-- \\
12 \\
8 \\
189\end{array}$ & $\begin{array}{l}35 \\
1 .- \\
68\end{array}$ & $\begin{array}{l}= \\
=- \\
.5 \\
3.9\end{array}$ & $\begin{array}{l}\bar{z} \\
\overline{3.1} \\
6.0\end{array}$ & $\begin{array}{l}=- \\
=- \\
1.1\end{array}$ \\
\hline & $\begin{array}{l}70-09-17 \\
70-10-22 \\
70-12-02 \\
71-01-14 \\
71-02-24\end{array}$ & $\begin{array}{l}28.5 \\
25.0 \\
23.5 \\
24.0 \\
23.5\end{array}$ & $\begin{array}{l}126 \\
252 \\
376 \\
370 \\
382\end{array}$ & $\begin{array}{r}8.0 \\
9.0 \\
9.0 \\
9.0 \\
11\end{array}$ & $\begin{array}{l}6.6 \\
7.6 \\
7.5 \\
7.5 \\
7.6\end{array}$ & $\begin{array}{l}405 \\
236 \\
224 \\
268 \\
222\end{array}$ & $69^{--}$ & $\begin{array}{l}-\overline{4.1} \\
\overline{-1.1} \\
--.\end{array}$ & $\begin{array}{r}0.3 \\
-- \\
6.0 \\
--\end{array}$ & $\begin{array}{l}-. \\
1.1 \\
0 . \\
-0\end{array}$ \\
\hline & $\begin{array}{l}71-03-31 \\
71-04-28 \\
71-06-15 \\
71-07-28 \\
71-09-21\end{array}$ & $\begin{array}{l}23.0 \\
25.0 \\
25.0 \\
25.0 \\
26.0\end{array}$ & $\begin{array}{l}367 \\
372 \\
380 \\
372 \\
372\end{array}$ & $\begin{array}{l}9.0 \\
9.0 \\
9.0 \\
9.0 \\
10\end{array}$ & $\begin{array}{l}7.6 \\
7 .-2 \\
7.6 \\
7.6\end{array}$ & $\begin{array}{r}228 \\
-- \\
=- \\
--\end{array}$ & $\begin{array}{l}= \\
= \\
= \\
=\end{array}$ & $\begin{array}{l}-- \\
-- \\
--\end{array}$ & $\begin{array}{l}=- \\
= \\
=\end{array}$ & $\begin{array}{l}=- \\
=- \\
=\end{array}$ \\
\hline & $\begin{array}{l}71-09-22 \\
71-10-28 \\
71-11-22 \\
71-12-27 \\
72-03-16\end{array}$ & $\begin{array}{l}= \\
=- \\
=-\end{array}$ & $\begin{array}{l}106 \\
104 \\
358 \\
452 \\
360\end{array}$ & ${ }_{11^{9.5}}^{--}$ & $\begin{array}{l}6.5 \\
6.0 \\
7.5 \\
-.-\end{array}$ & $\begin{array}{l}68 \\
45 \\
-8 \\
-8 \\
-0\end{array}$ & $\begin{array}{c}63^{--} \\
190^{--}\end{array}$ & $\begin{array}{l}=- \\
5.6 \\
49 \\
14\end{array}$ & $\begin{array}{l}-. \\
-.5 \\
8.1 \\
1.0\end{array}$ & $\begin{array}{r}-- \\
-. \\
.4 \\
1.6 \\
.7\end{array}$ \\
\hline & $\begin{array}{l}72-06-20 \\
73-02-01 \\
73-04-24 \\
73-08-09 \\
73-12-19\end{array}$ & $\begin{array}{l}= \\
=- \\
=-\end{array}$ & $\begin{array}{l}370 \\
370 \\
372 \\
381 \\
379\end{array}$ & $\begin{array}{c}9.6 \\
12 \\
8.0 \\
10 \\
10\end{array}$ & $\begin{array}{l}\overline{-.} \\
\overline{-} \\
=-\end{array}$ & $\begin{array}{l}= \\
= \\
= \\
=\end{array}$ & $\begin{array}{l}64 \\
70 \\
65 \\
65 \\
78\end{array}$ & $\begin{array}{l}5.4 \\
3.9 \\
4.1 \\
4.8 \\
4.9\end{array}$ & $\begin{array}{l}6.0 \\
5.9 \\
7.2 \\
7.4 \\
7.5\end{array}$ & $\begin{array}{r}.7 \\
.8 \\
.9 \\
.9 \\
1.8\end{array}$ \\
\hline 280238082342603 & $\begin{array}{l}70-01-07 \\
70-02-02 \\
70-09-17 \\
71-09-22 \\
71-10-28\end{array}$ & $\begin{array}{l}= \\
=- \\
=\end{array}$ & $\begin{array}{r}72 \\
45 \\
126 \\
106 \\
104\end{array}$ & $\begin{array}{r}12 \\
5.0 \\
8.0 \\
6.0 \\
8.0\end{array}$ & $\begin{array}{l}6.3 \\
-- \\
6.6 \\
6.5 \\
6.0\end{array}$ & $\begin{array}{r}-- \\
405 \\
68 \\
45\end{array}$ & $\begin{array}{l}= \\
=- \\
= \\
=\end{array}$ & $\begin{array}{l}= \\
=- \\
= \\
=\end{array}$ & $\begin{array}{l}= \\
= \\
=\end{array}$ & $\begin{array}{l}=- \\
=- \\
=\end{array}$ \\
\hline \multirow[t]{6}{*}{280238082342602} & $\begin{array}{l}71-12-27 \\
70-01-07 \\
70-02-02 \\
70-06-25 \\
70-08-04\end{array}$ & $\begin{array}{r}-- \\
19.5 \\
25.0 \\
28.0\end{array}$ & $\begin{array}{r}452 \\
102 \\
108 \\
188 \\
74\end{array}$ & $\begin{array}{r}8.0 \\
9.8 \\
5.5 \\
11 \\
6.2\end{array}$ & $\begin{array}{l}7.3 \\
6.3 \\
6.5 \\
6.9 \\
5.7\end{array}$ & $\begin{array}{r}-- \\
40 \\
48 \\
121 \\
28\end{array}$ & $\begin{array}{r}81 \\
6.1 \\
-- \\
--\end{array}$ & $\begin{array}{l}49 \\
1.2 \\
=-\end{array}$ & $\begin{array}{r}8.1 \\
2.3 \\
-- \\
--\end{array}$ & $\begin{array}{l}1.6 \\
-.2 \\
-. \\
-.\end{array}$ \\
\hline & $\begin{array}{l}70-09-17 \\
70-10-22 \\
70-12-03 \\
71-01-13 \\
71-02-26\end{array}$ & $\begin{array}{l}27.9 \\
25.5 \\
24.0 \\
22.0 \\
22.5\end{array}$ & $\begin{array}{r}94 \\
75 \\
111 \\
42 \\
54\end{array}$ & $\begin{array}{l}6.0 \\
4.0 \\
6.0 \\
6.0 \\
8.0\end{array}$ & $\begin{array}{l}6.8 \\
6.8 \\
6.2 \\
5.7 \\
6.2\end{array}$ & $\begin{array}{l}53 \\
25 \\
42 \\
14 \\
36\end{array}$ & $\begin{array}{r}-- \\
4.4 \\
2.9 \\
--\end{array}$ & $\begin{array}{l}-. \\
.9 \\
.5 \\
-.\end{array}$ & $\begin{array}{l}3.9 \\
3.2 \\
-.\end{array}$ & $\begin{array}{l}-5 \\
.3 \\
01 \\
-.\end{array}$ \\
\hline & $\begin{array}{l}71-03-30 \\
71-04-28 \\
71-06-14 \\
71-07-27 \\
71-09-22\end{array}$ & $\begin{array}{l}21.0 \\
22.0 \\
23.0 \\
25.0 \\
27.0\end{array}$ & $\begin{array}{r}33 \\
62 \\
57 \\
42 \\
288\end{array}$ & $\begin{array}{l}4.0 \\
4.0 \\
4.0 \\
4.0 \\
5.0\end{array}$ & $\begin{array}{l}6.3 \\
-.2 \\
6.2 \\
6.1 \\
6.6\end{array}$ & $\begin{array}{r}30 \\
-- \\
40 \\
40 \\
380\end{array}$ & $\begin{array}{l}= \\
= \\
= \\
=\end{array}$ & $\begin{array}{l}= \\
z \\
--\end{array}$ & $\begin{array}{l}= \\
= \\
=\end{array}$ & $\begin{array}{l}= \\
=- \\
= \\
=\end{array}$ \\
\hline & $\begin{array}{l}71-10-28 \\
71-11-22 \\
71-12-27 \\
72-01-27 \\
72-03-16\end{array}$ & $\begin{array}{l}= \\
= \\
=\end{array}$ & $\begin{array}{r}218 \\
245 \\
42 \\
27 \\
25\end{array}$ & $\begin{array}{l}6.0 \\
6.2 \\
6.0 \\
4.5 \\
5.0\end{array}$ & $\begin{array}{l}-- \\
6.1 \\
6.2 \\
-.\end{array}$ & $\begin{array}{r}=- \\
24 \\
128 \\
--\end{array}$ & $\begin{array}{l}42^{--} \\
2 .-\end{array}$ & $\begin{array}{l}3.6 \\
=- \\
1.6\end{array}$ & $\begin{array}{l}5.0 \\
-- \\
.0\end{array}$ & $\begin{array}{l}-0 \\
00 \\
-0 \\
.1\end{array}$ \\
\hline & $\begin{array}{l}72-06-21 \\
72-07-18 \\
72-08-08 \\
72-09-06 \\
72-10-26\end{array}$ & $\begin{array}{l}= \\
= \\
=\end{array}$ & $\begin{array}{l}32 \\
37 \\
-7 \\
48 \\
54\end{array}$ & $\begin{array}{l}5.0 \\
8.4 \\
-.5 \\
9.0\end{array}$ & $\begin{array}{l}=- \\
z \\
-\end{array}$ & $\begin{array}{l}= \\
= \\
z\end{array}$ & $\begin{array}{c}5.3 \\
13 \\
5.3 \\
3.4 \\
4.0\end{array}$ & $\begin{array}{r}1.7 \\
14 \\
.9 \\
.8 \\
.7\end{array}$ & $\begin{array}{l}4.0 \\
-.8 \\
4.2 \\
41\end{array}$ & $\begin{array}{l}.0 \\
.0 \\
.2 \\
.1 \\
.1\end{array}$ \\
\hline & $\begin{array}{l}73-02-01 \\
73-04-27 \\
73-08-13 \\
73-10-16 \\
73-12-20\end{array}$ & $\begin{array}{l}= \\
= \\
=\end{array}$ & $\begin{array}{l}45 \\
38 \\
47 \\
69 \\
62\end{array}$ & $\begin{array}{l}2.6 \\
7.9 \\
8.0 \\
9.7 \\
9.8\end{array}$ & $\begin{array}{l}6.4 \\
\square \\
= \\
=\end{array}$ & 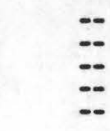 & $\begin{array}{l}3.2 \\
2.4 \\
2.8 \\
6.0 \\
3.0\end{array}$ & $\begin{array}{r}.7 \\
: 6 \\
: 7 \\
.9 \\
1.3\end{array}$ & $\begin{array}{l}3.7 \\
3.4 \\
4.5 \\
4.3 \\
5.6\end{array}$ & $\begin{array}{l}.2 \\
.1 \\
.0 \\
.1 \\
.6\end{array}$ \\
\hline \multirow[t]{3}{*}{280238082342601} & $\begin{array}{l}70-01-07 \\
70-02-02 \\
70-08-04 \\
70-09-17 \\
70-10-22\end{array}$ & $\begin{array}{r}=- \\
27.0 \\
27.5 \\
26.0\end{array}$ & $\begin{array}{r}258 \\
285 \\
324 \\
322 \\
3\end{array}$ & $\begin{array}{l}9.4 \\
6.0 \\
8.2 \\
7.0 \\
8.0\end{array}$ & $\begin{array}{l}7 .- \\
7.6 \\
7.7 \\
7.8 \\
7.5\end{array}$ & $\begin{array}{l}136 \\
190 \\
189 \\
202\end{array}$ & ${ }_{64}^{50}$ & $\begin{array}{r}3.1 \\
3.1 \\
4.1\end{array}$ & $\begin{array}{r}4.7 \\
\overline{0.1}\end{array}$ & $\begin{array}{l}1.3 \\
0 . \\
1.2\end{array}$ \\
\hline & $\begin{array}{l}70-12-03 \\
71-01-13 \\
71-02-26 \\
71-03-30 \\
71-04-28\end{array}$ & $\begin{array}{l}23.5 \\
22.0 \\
23.0 \\
21.0 \\
23.0\end{array}$ & $\begin{array}{l}402 \\
432 \\
438 \\
402 \\
418\end{array}$ & $\begin{array}{l}9.0 \\
8.0 \\
8.0 \\
8.0 \\
8.0\end{array}$ & $\begin{array}{r}7.5 \\
7.5 \\
7.7 \\
7.7 \\
-.\end{array}$ & $\begin{array}{l}252 \\
266 \\
270 \\
270 \\
--\end{array}$ & $\begin{array}{l}75^{--} \\
=- \\
=-\end{array}$ & $\begin{array}{l}5.0 \\
-- \\
=\end{array}$ & $\begin{array}{l}0.1 \\
=- \\
=-\end{array}$ & $\begin{array}{l}1.2 \\
=- \\
-.\end{array}$ \\
\hline & $\begin{array}{l}71-06-14 \\
71-07-27 \\
71-09-22 \\
71-10-28 \\
71-11-22\end{array}$ & $\begin{array}{r}24.0 \\
24.0 \\
26.0 \\
=-\end{array}$ & $\begin{array}{l}432 \\
420 \\
402 \\
478 \\
290\end{array}$ & $\begin{array}{r}-0 \\
8.0 \\
9.0 \\
11 \\
9.5\end{array}$ & $\begin{array}{l}7.6 \\
6.8 \\
7.6 \\
6.8 \\
-.\end{array}$ & $\begin{array}{r}270 \\
268 \\
296 \\
-. \\
-.\end{array}$ & $\begin{array}{l}= \\
= \\
49^{-}\end{array}$ & $\begin{array}{l}= \\
= \\
=-8\end{array}$ & $\begin{array}{l}= \\
= \\
6.5\end{array}$ & $\begin{array}{l}= \\
= \\
= \\
0\end{array}$ \\
\hline
\end{tabular}




\begin{tabular}{|c|c|c|c|c|c|c|c|c|c|c|}
\hline STATION NUMBER & $\begin{array}{l}\text { DATE } \\
\text { OF } \\
\text { SAMPLE }\end{array}$ & $\begin{array}{l}\text { TEMPER- } \\
\text { ATURE } \\
\text { (DEG C) }\end{array}$ & $\begin{array}{l}\text { SPE- } \\
\text { CIFIC } \\
\text { CON- } \\
\text { DUCT- } \\
\text { ANCE } \\
\text { (MICRO- } \\
\text { MHOS) }\end{array}$ & $\begin{array}{l}\text { CHLO- } \\
\text { RIOE, } \\
\text { DIS- } \\
\text { SOLVEO } \\
\text { (MG/L } \\
\text { AS CL) }\end{array}$ & $\begin{array}{c}P H \\
\text { (UNITS) }\end{array}$ & $\begin{array}{l}\text { ALKA- } \\
\text { LINITY } \\
\text { IMG/L } \\
\text { AS } \\
\text { CACO3) }\end{array}$ & $\begin{array}{l}\text { CALCIUM } \\
\text { OIS- } \\
\text { SOLVEO } \\
\text { (MG/L } \\
\text { AS CA) }\end{array}$ & $\begin{array}{l}\text { MAGNE- } \\
\text { SIUM, } \\
\text { DIS- } \\
\text { SOLVED } \\
\text { (MG/L } \\
\text { AS MG) }\end{array}$ & $\begin{array}{l}\text { SOUIUM, } \\
\text { DIS- } \\
\text { SOLVED } \\
\text { (MGLL } \\
\text { AS NA) }\end{array}$ & $\begin{array}{l}\text { POTAS- } \\
\text { SIUM, } \\
\text { DIS- } \\
\text { SOLVED } \\
\text { (MG/L } \\
\text { AS K) }\end{array}$ \\
\hline 280238082342601 & $\begin{array}{l}72-06-21 \\
72-07-18 \\
72-10-26 \\
73-02-01 \\
73-04-27\end{array}$ & $\begin{array}{l}= \\
=- \\
= \\
=\end{array}$ & $\begin{array}{l}410 \\
371 \\
418 \\
395 \\
348\end{array}$ & $\begin{array}{l}8.4 \\
11 \\
10 \\
10 \\
8.8\end{array}$ & $\begin{array}{l}=- \\
0 \\
7.6 \\
0\end{array}$ & $\begin{array}{l}= \\
=- \\
=\end{array}$ & $\begin{array}{l}85^{--} \\
82 \\
73 \\
73\end{array}$ & $\begin{array}{l}4.0 \\
19 \\
4.2 \\
3.6 \\
3.7\end{array}$ & $\begin{array}{l}9.0 \\
9.0 \\
7.7 \\
7.4 \\
8.5\end{array}$ & $\begin{array}{l}1.2 \\
.4 \\
1.3 \\
1.1 \\
1.3\end{array}$ \\
\hline 280238082335701 & $\begin{array}{l}73-08-13 \\
73-10-16 \\
73-12-20 \\
73-01-26 \\
73-02-01\end{array}$ & $\begin{array}{l}= \\
=- \\
=-\end{array}$ & $\begin{array}{l}385 \\
420 \\
375 \\
375\end{array}$ & $\begin{array}{c}7.0 \\
11 \\
7.9 \\
10\end{array}$ & $\begin{array}{l}= \\
\because \\
=- \\
7.6\end{array}$ & 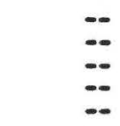 & $\begin{array}{l}67 \\
=- \\
71\end{array}$ & $\begin{array}{c}3.5 \\
0- \\
=- \\
3.7\end{array}$ & $\begin{array}{l}7.8 \\
=- \\
=- \\
6.0\end{array}$ & $\begin{array}{l}1.1 \\
\square \\
=0 \\
.7\end{array}$ \\
\hline & $\begin{array}{l}73-04-25 \\
73-08-13 \\
73-10-09 \\
73-10-16 \\
73-12-21\end{array}$ & $\begin{array}{l}= \\
=- \\
=-\end{array}$ & $\begin{array}{l}379 \\
400 \\
122 \\
547 \\
491\end{array}$ & $\begin{array}{l}7.0 \\
13 \\
53^{--} \\
39\end{array}$ & $\begin{array}{l}7.7 \\
- \\
\because- \\
-\end{array}$ & $\begin{array}{c}184 \\
=- \\
=-\end{array}$ & $\begin{array}{l}67 \\
66 \\
64 \\
76\end{array}$ & $\begin{array}{r}3.9 \\
3.8 \\
3.8 \\
4.4\end{array}$ & $\begin{array}{l}10 \\
10^{7.2} \\
43^{-\cdots}\end{array}$ & $\begin{array}{l}.8 \\
.6 \\
0 . \\
0.0\end{array}$ \\
\hline 280238082342205 & $\begin{array}{l}70-08-04 \\
70-09-18 \\
70-10-26 \\
70-12-03 \\
71-01-13\end{array}$ & $\begin{array}{l}26.0 \\
26.0 \\
25.0 \\
23.0 \\
21.0\end{array}$ & $\begin{array}{l}360 \\
312 \\
330 \\
368 \\
370\end{array}$ & $\begin{array}{l}9.0 \\
11 \\
11 \\
10 \\
9.0\end{array}$ & $\begin{array}{l}7.5 \\
7.3 \\
7.7 \\
7.3 \\
7.6\end{array}$ & $\begin{array}{l}206 \\
168 \\
173 \\
214 \\
218\end{array}$ & $\begin{array}{l}\ddot{-} \\
= \\
= \\
-\end{array}$ & $\begin{array}{l}\because \\
\because \\
\therefore \\
-\infty\end{array}$ & $\begin{array}{l}-- \\
- \\
-\end{array}$ & 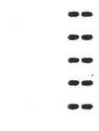 \\
\hline & $\begin{array}{l}71-02-24 \\
71-03-31 \\
71-04-28 \\
71-06-14 \\
71-07-27\end{array}$ & $\begin{array}{l}23.0 \\
23.0 \\
21.5 \\
22.5 \\
24.5\end{array}$ & $\begin{array}{l}372 \\
348 \\
378 \\
294 \\
324\end{array}$ & $\begin{array}{l}8.0 \\
8.0 \\
9.0 \\
6.0 \\
6.0\end{array}$ & $\begin{array}{r}7.5 \\
7.5 \\
.-5 \\
7.4 \\
6.9\end{array}$ & $\begin{array}{l}232 \\
214 \\
196 \\
196 \\
220\end{array}$ & $\begin{array}{l}= \\
\ddot{-} \\
=\end{array}$ & $\begin{array}{l}= \\
= \\
=\end{array}$ & $\begin{array}{l}= \\
= \\
= \\
=\end{array}$ & 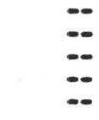 \\
\hline 280238082342204 & $\begin{array}{l}73-04-26 \\
73-12-20 \\
70-04-08 \\
70-09-18 \\
70-10-26\end{array}$ & $\begin{array}{l}=- \\
26.0 \\
27.0 \\
25.0\end{array}$ & $\begin{array}{l}341 \\
413 \\
100 \\
121 \\
118\end{array}$ & $\begin{array}{l}7.0 \\
7.9 \\
7.0 \\
6.0 \\
5.0\end{array}$ & $\begin{array}{l}-- \\
-- \\
6.4 \\
6.5 \\
6.8\end{array}$ & $\begin{array}{l}-0 \\
-8 \\
61 \\
78 \\
92\end{array}$ & $\begin{array}{r}56 \\
69 \\
=- \\
=-\end{array}$ & $\begin{array}{c}31 \\
1.9 \\
\ldots \\
\ldots\end{array}$ & $\begin{array}{r}8.0 \\
9.5 \\
=- \\
=- \\
=-\end{array}$ & $\begin{array}{r}09 \\
1.4 \\
=- \\
-0\end{array}$ \\
\hline & $\begin{array}{l}70-12-03 \\
71=01-13 \\
71-03-31 \\
71-04-28 \\
71-06-14\end{array}$ & $\begin{array}{l}22.0 \\
20.5 \\
20.0 \\
21.0 \\
23.0\end{array}$ & $\begin{array}{r}147 \\
63 \\
95 \\
121 \\
92\end{array}$ & $\begin{array}{l}8.0 \\
4.0 \\
4.0 \\
5.0 \\
4.0\end{array}$ & $\begin{array}{l}6.6 \\
6.3 \\
6.6 \\
-.5 \\
6.5\end{array}$ & $\begin{array}{l}98 \\
42 \\
90 \\
-5 \\
60\end{array}$ & $\begin{array}{l}= \\
= \\
= \\
=\end{array}$ & 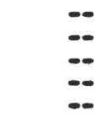 & $\begin{array}{l}-- \\
-\infty \\
-\infty\end{array}$ & 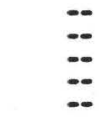 \\
\hline 280238082342203 & $\begin{array}{l}71-07-27 \\
71-11-22 \\
72-07-19 \\
73-04-27 \\
70-02-27\end{array}$ & $\begin{array}{r}25.0 \\
=- \\
=- \\
=-\end{array}$ & $\begin{array}{r}92 \\
259 \\
382 \\
420 \\
351\end{array}$ & $\begin{array}{c}5.0 \\
5.8 \\
12 \\
10 \\
9.0\end{array}$ & $\begin{array}{l}6.5 \\
=- \\
=- \\
=\end{array}$ & $\begin{array}{l}84 \\
=- \\
=-\end{array}$ & $\begin{array}{c}4.7 \\
130^{-0} \\
74 \\
\ldots\end{array}$ & $\begin{array}{c}3.2 \\
26 \\
4.0 \\
-0\end{array}$ & $\begin{array}{l}3.5 \\
-.5 \\
7.1 \\
-.\end{array}$ & $\begin{array}{l}-0 \\
.0 \\
: 9 \\
-0\end{array}$ \\
\hline & $\begin{array}{l}70-08-04 \\
70-09-18 \\
70-10-26 \\
70-12-03 \\
71-01-13\end{array}$ & $\begin{array}{l}22.5 \\
25.0 \\
28.0 \\
25.5 \\
21.0\end{array}$ & $\begin{array}{l}409 \\
395 \\
390 \\
394 \\
370\end{array}$ & $\begin{array}{l}9.0 \\
10 \\
11 \\
1.0 \\
9.0\end{array}$ & $\begin{array}{l}7.3 \\
7.5 \\
7.7 \\
7.2 \\
7.3\end{array}$ & $\begin{array}{l}335 \\
240 \\
243 \\
240 \\
355\end{array}$ & $\begin{array}{l}= \\
= \\
= \\
=\end{array}$ & $\begin{array}{l}=- \\
= \\
= \\
=\end{array}$ & $\begin{array}{l}= \\
\ddot{-} \\
-\infty\end{array}$ & $\begin{array}{l}-\infty \\
\ddot{-} \\
-\end{array}$ \\
\hline & $\begin{array}{l}71-02-24 \\
71-03-31 \\
71-04-28 \\
71-06-14 \\
71-07-27\end{array}$ & $\begin{array}{l}23.0 \\
22.5 \\
21.5 \\
21.5 \\
22.5\end{array}$ & $\begin{array}{l}392 \\
374 \\
350 \\
390 \\
389\end{array}$ & $\begin{array}{l}9.0 \\
9.0 \\
7.0 \\
8.0 \\
8.0\end{array}$ & $\begin{array}{l}7.4 \\
7.6 \\
7.4 \\
7.1\end{array}$ & $\begin{array}{l}720 \\
244 \\
240 \\
268\end{array}$ & $\begin{array}{l}= \\
= \\
= \\
=\end{array}$ & $\begin{array}{l}= \\
= \\
= \\
=\end{array}$ & $\begin{array}{l}= \\
= \\
= \\
=\end{array}$ & $\begin{array}{l}\square \\
\square \\
=\end{array}$ \\
\hline & $\begin{array}{l}71-09-22 \\
71-10-29 \\
72-01-27 \\
72-07-20 \\
73-04-26\end{array}$ & $\begin{array}{r}23.0 \\
0 . \\
20.0 \\
\ldots\end{array}$ & $\begin{array}{l}380 \\
379 \\
378 \\
378 \\
387\end{array}$ & $\begin{array}{c}8.0 \\
12 \\
9.0 \\
9.0 \\
7.7\end{array}$ & $\begin{array}{r}7.4 \\
7.2 \\
7.7 \\
-. \\
=-\end{array}$ & $\begin{array}{r}432 \\
236 \\
238 \\
-- \\
--\end{array}$ & $\begin{array}{l}= \\
= \\
68\end{array}$ & $\begin{array}{l}= \\
= \\
= \\
4.3\end{array}$ & $\begin{array}{l}= \\
=- \\
=1\end{array}$ & 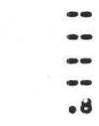 \\
\hline 280240082340601 & $\begin{array}{l}71-12-28 \\
72-01-26 \\
72-03-16 \\
72-07-17 \\
72-07-19\end{array}$ & $\begin{array}{l}= \\
= \\
= \\
=\end{array}$ & $\begin{array}{l}380 \\
370 \\
380 \\
146 \\
382\end{array}$ & $\begin{array}{l}9.0 \\
10^{20} \\
13\end{array}$ & 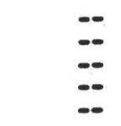 & $\begin{array}{l}= \\
=- \\
=\end{array}$ & $\begin{array}{l}68 \\
70^{-0} \\
31 \\
78\end{array}$ & $\begin{array}{r}122 \\
3.8 \\
3.6 \\
17\end{array}$ & $\begin{array}{l}6.1 \\
-.5 \\
1.5 \\
-. \\
-.\end{array}$ & $\begin{array}{l}.8 \\
-8 \\
.3 \\
.2 \\
-0\end{array}$ \\
\hline & $\begin{array}{l}72-10-25 \\
72-10-26 \\
73-02-01 \\
73-04-23 \\
73-04-25\end{array}$ & 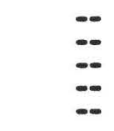 & $\begin{array}{r}377 \\
54 \\
40 \\
387 \\
122\end{array}$ & $\begin{array}{l}7.2 \\
3.4 \\
5.5 \\
8.0 \\
4.5\end{array}$ & $\begin{array}{l}0 \\
0.1 \\
=\end{array}$ & $\begin{array}{l}= \\
= \\
=\end{array}$ & $\begin{array}{l}64 \\
4.0 \\
3.8 \\
67 \\
14\end{array}$ & $\begin{array}{r}3.9 \\
.7 \\
.4 \\
4.1 \\
1.0\end{array}$ & $\begin{array}{l}6.3 \\
4.1 \\
3.9 \\
7.4 \\
6.2\end{array}$ & $\begin{array}{l}.7 \\
-. \\
.3 \\
.8 \\
.3\end{array}$ \\
\hline & $\begin{array}{l}73-08-09 \\
73-08-13 \\
73-10-15 \\
73-10-16 \\
73-12-19\end{array}$ & $\begin{array}{l}= \\
\ddot{-} \\
=\end{array}$ & $\begin{array}{r}388 \\
110 \\
384 \\
36 \\
383\end{array}$ & $\begin{array}{l}8.0 \\
9.0 \\
6.2 \\
8.4\end{array}$ & $\begin{array}{l}= \\
= \\
=\end{array}$ & $\begin{array}{l}= \\
= \\
=\end{array}$ & $\begin{array}{l}69 \\
16 \\
72 \\
2.8 \\
63\end{array}$ & $\begin{array}{l}3.9 \\
.8 \\
3.6 \\
.3 \\
5.8\end{array}$ & $\begin{array}{l}0.4 \\
4.4 \\
6.4 \\
4.3 \\
8.1\end{array}$ & $\begin{array}{l}.8 \\
.4 \\
.0 \\
.0 \\
.8\end{array}$ \\
\hline 280240082340602 & $\begin{array}{l}73-12-20 \\
73-01-02 \\
73-04-25 \\
73-08-13 \\
73-10-16\end{array}$ & 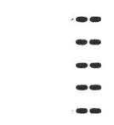 & $\begin{array}{r}36 \\
375 \\
423 \\
460 \\
435\end{array}$ & $\begin{array}{r}4.8 \\
=-8 \\
7.4 \\
0 .\end{array}$ & $\begin{array}{r}\square .9 \\
\because- \\
\therefore\end{array}$ & $\begin{array}{l}= \\
= \\
= \\
=\end{array}$ & $\begin{array}{l}4.5 \\
68 \\
76 \\
73 \\
79\end{array}$ & $\begin{array}{l}.44 \\
4.0 \\
4.8 \\
5.2 \\
5.3\end{array}$ & $\begin{array}{l}4.5 \\
6.3 \\
7.8 \\
7.2 \\
.6\end{array}$ & $\begin{array}{r}.3 \\
.8 \\
1.2 \\
1.3 \\
. .0\end{array}$ \\
\hline $\begin{array}{l}280240082341202 \\
280240082341201\end{array}$ & $\begin{array}{l}73-12-19 \\
73-08-10 \\
73-12-19 \\
73-08-01 \\
73-08-10\end{array}$ & $\begin{array}{l}= \\
= \\
=\end{array}$ & $\begin{array}{l}411 \\
385 \\
392 \\
380 \\
270\end{array}$ & $\begin{array}{l}7.9 \\
8.0 \\
9.0 \\
10 \\
10\end{array}$ & $\begin{array}{l}= \\
\therefore \\
=\end{array}$ & $\begin{array}{l}= \\
= \\
= \\
=\end{array}$ & $\begin{array}{l}68 \\
72 \\
65 \\
67 \\
50\end{array}$ & $\begin{array}{l}6.0 \\
4.4 \\
5.1 \\
5.2 \\
3.7\end{array}$ & $\begin{array}{c}7.0 \\
6.3 \\
6.1 \\
12 \\
11\end{array}$ & $\begin{array}{r}2.2 \\
.8 \\
1.5 \\
.3 \\
.5\end{array}$ \\
\hline
\end{tabular}




\begin{tabular}{|c|c|c|c|c|c|c|c|c|c|c|}
\hline STATION NUMBER & $\begin{array}{c}\text { DATE } \\
\text { OF } \\
\text { SAMPLE }\end{array}$ & $\begin{array}{l}\text { TEMPER- } \\
\text { ATURE } \\
\text { (DEG C) }\end{array}$ & $\begin{array}{l}\text { SPE- } \\
\text { CIF IC } \\
\text { CON- } \\
\text { DUCT- } \\
\text { ANCE } \\
\text { (MICRO- } \\
\text { MHOS) }\end{array}$ & $\begin{array}{l}\text { CHLO- } \\
\text { RIDE, } \\
\text { DIS- } \\
\text { SOLVED } \\
\text { (MG/L } \\
\text { AS CL) }\end{array}$ & $\begin{array}{c}\text { PH } \\
\text { (UNITS) }\end{array}$ & $\begin{array}{l}\text { ALKA- } \\
\text { LINITY } \\
\text { (MG/L } \\
\text { AS } \\
\text { CACO3) }\end{array}$ & $\begin{array}{l}\text { CALCIUM } \\
\text { DIS- } \\
\text { SOLVED } \\
\text { (MGUL } \\
\text { AS CA) }\end{array}$ & $\begin{array}{l}\text { MAGNE- } \\
\text { SIUM, } \\
\text { DIS- } \\
\text { SOLVED } \\
\text { (MG/L } \\
\text { AS MG) }\end{array}$ & $\begin{array}{l}\text { SOUIUM, } \\
\text { OIS- } \\
\text { SOLVED } \\
\text { (MGIL } \\
\text { AS NA) }\end{array}$ & $\begin{array}{l}\text { POTAS- } \\
\text { SIUM, } \\
\text { DIS- } \\
\text { SOLVEU } \\
\text { (MGAL } \\
\text { AS K) }\end{array}$ \\
\hline $\begin{array}{l}280240082341201 \\
280240082335901\end{array}$ & $\begin{array}{l}73-10-16 \\
73-12-19 \\
72-07-17 \\
72-10-26 \\
73-02-01\end{array}$ & $\begin{array}{l}= \\
= \\
= \\
=\end{array}$ & $\begin{array}{r}123 \\
199 \\
73 \\
68 \\
43\end{array}$ & $\begin{array}{l}9.5 \\
7.8 \\
8.4 \\
6.0 \\
11\end{array}$ & $\begin{array}{l}= \\
=- \\
=- \\
6.4\end{array}$ & $\begin{array}{l}= \\
=- \\
=\end{array}$ & $\begin{array}{c}18 \\
30 \\
4.2 \\
5.5 \\
2.3\end{array}$ & $\begin{array}{l}1.1 \\
4.8 \\
1.3 \\
1.0 \\
.9\end{array}$ & $\begin{array}{l}9.6 \\
8.6 \\
4.5 \\
6.3\end{array}$ & $\begin{array}{l}.5 \\
.5 \\
.2 \\
.0 \\
.3\end{array}$ \\
\hline $\begin{array}{l}280240082335902 \\
280239082343503\end{array}$ & $\begin{array}{l}73-04-25 \\
73-02-01 \\
73-04-25 \\
70-02-05 \\
72-06-22\end{array}$ & $\begin{array}{r}= \\
= \\
21.0 \\
19.0\end{array}$ & $\begin{array}{r}104 \\
510 \\
467 \\
67 \\
208\end{array}$ & $\begin{array}{c}6.5 \\
10 \\
7.0 \\
9.0 \\
7.0\end{array}$ & $\begin{array}{r}7.9 \\
6.5 \\
-. .\end{array}$ & $\begin{array}{l}-- \\
-- \\
18 \\
--\end{array}$ & $\begin{array}{r}11 \\
110 \\
85 \\
19\end{array}$ & $\begin{array}{l}1.7 \\
8.2 \\
5.8 \\
-2 \\
3.2\end{array}$ & $\begin{array}{l}6.2 \\
6.8 \\
7.2 \\
-. \\
5.0\end{array}$ & $\begin{array}{l}.2 \\
.8 \\
.7 \\
1.7\end{array}$ \\
\hline 280239082343502 & $\begin{array}{l}73-02-01 \\
72-06-20 \\
72-07-18 \\
72-08-08 \\
72-09-06\end{array}$ & $\begin{array}{l}=- \\
=- \\
=\end{array}$ & $\begin{array}{l}375 \\
314 \\
328 \\
240 \\
265\end{array}$ & $\begin{array}{l}11 \\
7.8 \\
13 \\
10^{--}\end{array}$ & $\begin{array}{l}7.9 \\
=- \\
=- \\
=-\end{array}$ & $\begin{array}{l}=- \\
=- \\
--\end{array}$ & $\begin{array}{c}68 \\
b b \\
160^{-0} \\
140^{--}\end{array}$ & $\begin{array}{r}4.1 \\
5.6 \\
3.9 \\
-.9\end{array}$ & $\begin{array}{c}0.0 \\
=- \\
=- \\
0.2\end{array}$ & $\begin{array}{l}.8 \\
.9 \\
.6 \\
-. \\
-.\end{array}$ \\
\hline 280239082343501 & $\begin{array}{l}72-10-25 \\
73-04-24 \\
70-02-05 \\
72-06-20 \\
72-07-18\end{array}$ & $\begin{array}{r}-- \\
21.0 \\
--\end{array}$ & $\begin{array}{l}285 \\
278 \\
318 \\
370 \\
386\end{array}$ & $\begin{array}{l}7.6 \\
8.0 \\
13 \\
11 \\
15\end{array}$ & $\begin{array}{l}-- \\
8.3 \\
-- \\
-\end{array}$ & $\begin{array}{r}-- \\
214 \\
-- \\
--\end{array}$ & $\begin{array}{c}48 \\
47 \\
64 \\
150\end{array}--$ & $\begin{array}{l}3.4 \\
3.5 \\
-.5 \\
7.2 \\
5.7\end{array}$ & $\begin{array}{l}5.5 \\
6.8 \\
-. . \\
7.0 \\
-.-\end{array}$ & $\begin{array}{r}2.1 \\
.5 \\
. . \\
.4 \\
. .\end{array}$ \\
\hline 280240082342601 & $\begin{array}{l}72-10-25 \\
73-04-24 \\
73-12-19 \\
70-01-08 \\
70-02-03\end{array}$ & $\begin{array}{r}=- \\
=- \\
19.0 \\
19.0\end{array}$ & $\begin{array}{l}377 \\
387 \\
372 \\
224 \\
309\end{array}$ & $\begin{array}{l}9.8 \\
10^{\circ} \\
12 \\
11 \\
9.8\end{array}$ & $\begin{array}{l}-- \\
=- \\
8.2 \\
7.8\end{array}$ & $\begin{array}{l}=- \\
=- \\
150 \\
181\end{array}$ & $\begin{array}{r}140 \\
66 \\
78 \\
--\end{array}$ & $\begin{array}{r}5.2 \\
4.7 \\
5.3 \\
-. \\
--\end{array}$ & $\begin{array}{r}6.6 \\
7.6 \\
7.3 \\
-.- \\
--\end{array}$ & $\begin{array}{r}.8 \\
.99 \\
1.3 \\
-8 \\
--\end{array}$ \\
\hline & $\begin{array}{l}70-09-17 \\
70-10-26 \\
70-12-03 \\
71-01-13 \\
71-02-26\end{array}$ & $\begin{array}{l}27.0 \\
26.0 \\
23.0 \\
22.5 \\
23.0\end{array}$ & $\begin{array}{l}287 \\
290 \\
286 \\
376 \\
368\end{array}$ & $\begin{array}{l}10 \\
10 \\
10^{--} \\
8.0\end{array}$ & $\begin{array}{l}7.5 \\
7.5 \\
7.4 \\
7.5 \\
7.5\end{array}$ & $\begin{array}{l}164 \\
156 \\
164 \\
296 \\
234\end{array}$ & $\begin{array}{l}= \\
=- \\
09\end{array}$ & $\begin{array}{l}= \\
=- \\
3.4 \\
=-\end{array}$ & $\begin{array}{l}=- \\
=- \\
6.0 \\
--\end{array}$ & $\begin{array}{l}- \\
- \\
1.0 \\
--\end{array}$ \\
\hline & $\begin{array}{l}71-03-30 \\
71-04-28 \\
71-06-14 \\
71-07-27 \\
71-12-27\end{array}$ & $\begin{array}{r}22.0 \\
22.0 \\
32.0 \\
24.0 \\
. .\end{array}$ & $\begin{array}{l}369 \\
368 \\
372 \\
386 \\
366\end{array}$ & $\begin{array}{l}9.0 \\
8.0 \\
10 \\
10 \\
11\end{array}$ & $\begin{array}{l}7.7 \\
7.5 \\
7.0 \\
7.8\end{array}$ & $\begin{array}{l}234 \\
232 \\
256 \\
385\end{array}$ & $\begin{array}{l}=- \\
=- \\
59^{--}\end{array}$ & 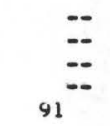 & $\begin{array}{l}-- \\
-- \\
=- \\
7.4\end{array}$ & $\begin{array}{l}= \\
\overline{-0} \\
1.3\end{array}$ \\
\hline & $\begin{array}{l}72-01-27 \\
72-03-15 \\
72-06-21 \\
72-07-18 \\
72-08-08\end{array}$ & $\begin{array}{l}=- \\
=- \\
=- \\
--\end{array}$ & $\begin{array}{l}364 \\
380 \\
410 \\
408 \\
440\end{array}$ & $\begin{array}{l}9.0 \\
9.7 \\
8.6 \\
12 \\
11\end{array}$ & $\begin{array}{l}= \\
= \\
8.0\end{array}$ & $\begin{array}{l}=- \\
=- \\
=-\end{array}$ & $\begin{array}{l}250^{--} \\
77 \\
91 \\
220\end{array}$ & $\begin{array}{c}10^{--} \\
5.5 \\
16^{-5} \\
5.8\end{array}$ & $\begin{array}{l}1 .- \\
7.5 \\
7.0 \\
6.1\end{array}$ & $\begin{array}{r}.0 \\
1.0 \\
.8 \\
1.2\end{array}$ \\
\hline 280240082342602 & $\begin{array}{l}72-09-06 \\
73-04-27 \\
72-10-26 \\
73-08-13 \\
73-12-20\end{array}$ & $\begin{array}{l}= \\
= \\
=\end{array}$ & $\begin{array}{r}440 \\
420 \\
117 \\
83 \\
93\end{array}$ & $\begin{array}{l}8.0 \\
10 \\
6.0 \\
6.0 \\
6.2\end{array}$ & $\begin{array}{l}= \\
= \\
= \\
=\end{array}$ & $\begin{array}{l}= \\
= \\
= \\
=\end{array}$ & $\begin{array}{r}120 \\
74 \\
21 \\
14 \\
17\end{array}$ & $\begin{array}{l}4.6 \\
4.0 \\
1.3 \\
1.0 \\
1.2\end{array}$ & $\begin{array}{l}0.1 \\
7.1 \\
4.1 \\
4.3 \\
4.9\end{array}$ & $\begin{array}{r}1.1 \\
.9 \\
.0 \\
.2 \\
1.3\end{array}$ \\
\hline $\begin{array}{l}280242082343503 \\
280242082343502\end{array}$ & $\begin{array}{l}70-02-06 \\
71-09-21 \\
72-01-26 \\
72-07-20 \\
70-01-22\end{array}$ & $\begin{array}{r}20.0 \\
33.0 \\
\ldots \\
\ldots \\
\ldots\end{array}$ & $\begin{array}{l}146 \\
109 \\
150 \\
150 \\
103\end{array}$ & $\begin{array}{l}16 \\
7.0 \\
7.5 \\
7.5 \\
7.4\end{array}$ & $\begin{array}{l}6.5 \\
6.7 \\
6.9 \\
7.5\end{array}$ & $\begin{array}{l}24 \\
52 \\
46 \\
-5\end{array}$ & $\begin{array}{l}= \\
= \\
=\end{array}$ & 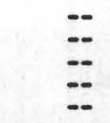 & $=$ & 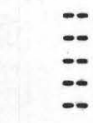 \\
\hline & $\begin{array}{l}70-02-06 \\
71-03-31 \\
71-04-28 \\
71-06-15 \\
71-09-21\end{array}$ & $\begin{array}{l}21.0 \\
22.0 \\
26.0 \\
23.5 \\
27.0\end{array}$ & $\begin{array}{r}238 \\
98 \\
105 \\
100 \\
118\end{array}$ & $\begin{array}{l}20 \\
7.0 \\
9.0 \\
7.0 \\
8.0\end{array}$ & $\begin{array}{l}7.1 \\
6.8 \\
6.4 \\
6.5\end{array}$ & $\begin{array}{r}116 \\
60 \\
-- \\
76 \\
68\end{array}$ & $\begin{array}{l}= \\
= \\
=\end{array}$ & $\begin{array}{l}= \\
= \\
=\end{array}$ & $\begin{array}{l}=- \\
=- \\
=-\end{array}$ & 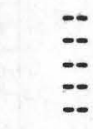 \\
\hline & $\begin{array}{l}71-10-28 \\
72-03-16 \\
72-06-20 \\
72-07-18 \\
72-08-08\end{array}$ & $\begin{array}{l}= \\
\therefore \\
-\therefore\end{array}$ & $\begin{array}{r}130 \\
230 \\
82 \\
240 \\
70\end{array}$ & $\begin{array}{r}8.0 \\
8.4 \\
11 \\
-.\end{array}$ & $\begin{array}{l}6.2 \\
=- \\
=-\end{array}$ & $\begin{array}{l}138 \\
=- \\
=- \\
--\end{array}$ & $\begin{array}{l}36^{--} \\
5.6 \\
10^{\circ} \\
37\end{array}$ & $\begin{array}{l}-. \\
3.8 \\
2.4 \\
3.3\end{array}$ & $\begin{array}{r}-.5 \\
.5 \\
3.5 \\
3.7\end{array}$ & $\begin{array}{l}-5 \\
.5 \\
.4 \\
.4 \\
-.\end{array}$ \\
\hline & $\begin{array}{l}72-09-06 \\
72-10-25 \\
73-02-01 \\
73-04-24 \\
73-08-13\end{array}$ & $\begin{array}{l}\square \\
\square \\
=\end{array}$ & $\begin{array}{r}90 \\
156 \\
80 \\
224 \\
155\end{array}$ & $\begin{array}{r}12 \\
4.0 \\
8.0 \\
7.8 \\
7.0\end{array}$ & $\begin{array}{l}= \\
= \\
= \\
=\end{array}$ & $\begin{array}{l}= \\
=- \\
=-\end{array}$ & $\begin{array}{l}42 \\
26 \\
17 \\
41 \\
19\end{array}$ & $\begin{array}{l}3.1 \\
1.9 \\
1.5 \\
3.2 \\
1.8\end{array}$ & $\begin{array}{l}3.5 \\
3.5 \\
3.7 \\
7.4 \\
5.0\end{array}$ & $\begin{array}{l}12 \\
4.0 \\
8.0 \\
7.8 \\
7.0\end{array}$ \\
\hline 280242082343501 & $\begin{array}{l}73-10-15 \\
73-12-20 \\
70-02-06 \\
71-03-31 \\
71-04-28\end{array}$ & $\begin{array}{r}=- \\
23.0 \\
23.0 \\
23.0\end{array}$ & $\begin{array}{l}191 \\
281 \\
324 \\
372 \\
374\end{array}$ & $\begin{array}{c}17 \\
38 \\
13 \\
10 \\
6.0\end{array}$ & $\begin{array}{l}=- \\
7.9 \\
7.4 \\
-.\end{array}$ & $\begin{array}{r}-- \\
222 \\
302 \\
--\end{array}$ & $\begin{array}{r}28 \\
38 \\
=- \\
=\end{array}$ & $\begin{array}{r}2.1 \\
3.7 \\
=- \\
=-\end{array}$ & $\begin{array}{r}16.1 \\
=- \\
=-\end{array}$ & $\begin{array}{r}17 \\
1.0 \\
0- \\
0\end{array}$ \\
\hline & $\begin{array}{l}71-06-15 \\
71-07-28 \\
71-09-21 \\
71-10-28 \\
72-03-16\end{array}$ & $\begin{array}{l}23.0 \\
24.0 \\
25.0 \\
24.0 \\
\ldots\end{array}$ & $\begin{array}{l}370 \\
380 \\
409 \\
372 \\
360\end{array}$ & $\begin{array}{l}10 \\
9.0 \\
10 \\
12 \\
9.8\end{array}$ & $\begin{array}{l}7.2 \\
7.6 \\
8.0 \\
7.4 \\
. .0\end{array}$ & $\begin{array}{r}228 \\
260 \\
320 \\
431 \\
--\end{array}$ & $230^{=}$ & $=$ & 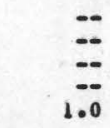 & 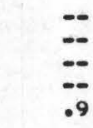 \\
\hline & $\begin{array}{l}72-06-20 \\
72-07-18 \\
72-08-08 \\
72-09-06 \\
72-10-25\end{array}$ & $\begin{array}{l}= \\
z \\
=\end{array}$ & $\begin{array}{l}372 \\
380 \\
370 \\
375 \\
377\end{array}$ & $\begin{array}{l}9.0 \\
12 \\
8.0 \\
9.0 \\
7.0\end{array}$ & $\begin{array}{l}= \\
= \\
=\end{array}$ & $\begin{array}{l}=- \\
= \\
=\end{array}$ & $\begin{array}{r}65 \\
98 \\
72 \\
72 \\
240\end{array}$ & $\begin{array}{l}8.3 \\
-.5 \\
4.6 \\
4.5 \\
6.1\end{array}$ & $\begin{array}{l}6.0 \\
6.5 \\
6.1 \\
0.8\end{array}$ & $\begin{array}{r}.4 \\
.4 \\
.8 \\
.8 \\
1.0\end{array}$ \\
\hline
\end{tabular}


TABLE 6A, --WATER-QUALITY DATA FOR GROUND-WATER SITES AT THE ROCKY CREEK LANDFilL--CONTINUED

\begin{tabular}{|c|c|c|c|c|c|c|c|c|c|c|}
\hline STATION NUMBER & $\begin{array}{c}\text { DATE } \\
\text { OF } \\
\text { SAMPLE }\end{array}$ & $\begin{array}{l}\text { TEMPER- } \\
\text { ATURE } \\
\text { (DEG C) }\end{array}$ & $\begin{array}{l}\text { SPE- } \\
\text { CIF IC } \\
\text { CON- } \\
\text { OUCT- } \\
\text { ANCE } \\
\text { (MICRO- } \\
\text { MHOS) }\end{array}$ & $\begin{array}{l}\text { CHLO- } \\
\text { RIDE, } \\
\text { OIS- } \\
\text { SOLVED } \\
\text { (MG/L } \\
\text { AS CL) }\end{array}$ & $\begin{array}{c}\text { PH } \\
\text { (UNITS) }\end{array}$ & $\begin{array}{l}\text { ALLA- } \\
\text { LINITY } \\
\text { (MGGL } \\
\text { AS } \\
\text { CACO3) }\end{array}$ & $\begin{array}{l}\text { CALCIUM } \\
\text { OIS- } \\
\text { SOLVED } \\
\text { (MG/L } \\
\text { AS CA) }\end{array}$ & $\begin{array}{l}\text { MAGNE- } \\
\text { SIUM, } \\
\text { DIS- } \\
\text { SOLVED } \\
\text { (MG/L } \\
\text { AS MG) }\end{array}$ & $\begin{array}{l}\text { SOUIUM, } \\
\text { DIS- } \\
\text { SOLVDED } \\
\text { (MG/L } \\
\text { AS NA) }\end{array}$ & $\begin{array}{l}\text { POTAS- } \\
\text { SIUM, } \\
\text { OIS- } \\
\text { SOLVED } \\
\text { (MG LL } \\
\text { AS K) }\end{array}$ \\
\hline \multirow[t]{2}{*}{280242082343501} & $\begin{array}{l}73-04-24 \\
73-08=09\end{array}$ & $=$ & $\begin{array}{l}382 \\
380\end{array}$ & $\begin{array}{l}8.2 \\
6.0\end{array}$ & $=$ & $=$ & $\begin{array}{l}67 \\
70\end{array}$ & $\begin{array}{l}4.5 \\
4.5\end{array}$ & $\begin{array}{l}7.3 \\
6.4\end{array}$ & .8 \\
\hline & $\begin{array}{l}73-10-15 \\
73-12-20\end{array}$ & $=$ & $\begin{array}{l}376 \\
395\end{array}$ & $\begin{array}{l}10 \\
10\end{array}$ & $=$ & $=$ & $75^{--}$ & 5.3 & 7.1 & 1.5 \\
\hline 280245082342701 & $71-10-28$ & -- & & & 5.4 & 494 & 245 & & & 42 \\
\hline \multirow{14}{*}{280242082342601} & $\begin{array}{l}72-01-27 \\
72-03-15\end{array}$ & $=$ & $\begin{array}{l}5400 \\
3300\end{array}$ & 100. & 5.1 & 486 & $=$ & $=$ & $140^{--}$ & $80^{--}$ \\
\hline & $\begin{array}{l}70-02-02 \\
70-09-17\end{array}$ & $\begin{array}{l}20.0 \\
27: 0\end{array}$ & $\begin{array}{l}108 \\
237\end{array}$ & $\begin{array}{l}5.0 \\
7.0\end{array}$ & $\begin{array}{l}6.3 \\
7.5\end{array}$ & $\begin{array}{r}59 \\
129\end{array}$ & $=$ & $\because$ & $=$ & $=-$ \\
\hline & $70-10-26$ & 25.5 & & 7.0 & 7.6 & 136 & -- & -- & -- & -- \\
\hline & $\begin{array}{l}70-12-03 \\
71-01-13\end{array}$ & $\begin{array}{l}23.0 \\
22.0\end{array}$ & $\begin{array}{l}249 \\
270\end{array}$ & $\begin{array}{l}8.0 \\
8.0\end{array}$ & $\begin{array}{l}7.1 \\
7.4\end{array}$ & $\begin{array}{l}134 \\
154\end{array}$ & $=$ & $=$ & $=$ & $\because$ \\
\hline & $\begin{array}{l}71-02-26 \\
71-03-30\end{array}$ & $\begin{array}{l}22.0 \\
20.0\end{array}$ & $\begin{array}{l}368 \\
260\end{array}$ & $\begin{array}{l}9.0 \\
9.0\end{array}$ & $\begin{array}{l}0.6 \\
7.8\end{array}$ & $\begin{array}{r}32 \\
230\end{array}$ & $=$ & $=$ & $=$ & $=$ \\
\hline & $71-04-28$ & 22.0 & 368 & 8.0 & -- & -- & -- & -- & -- & -- \\
\hline & $\begin{array}{l}71-06-14 \\
71=07-27\end{array}$ & $\begin{array}{l}25.5 \\
26.0\end{array}$ & $\begin{array}{l}375 \\
385\end{array}$ & $\begin{array}{l}10 \\
10\end{array}$ & $\begin{array}{l}7.5 \\
6.2\end{array}$ & $\begin{array}{r}210 \\
36\end{array}$ & $=$ & $=$ & $=$ & $=-$ \\
\hline & $71-10-28$ & $\ddot{-}$ & 367 & 12 & 7.6 & 234 & -- & $-\overline{3}$ & 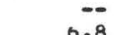 & - \\
\hline & $\begin{array}{l}71-12-27 \\
72-01-27\end{array}$ & $\overline{-}$ & $\begin{array}{l}380 \\
375\end{array}$ & $\begin{array}{l}10 \\
9.0\end{array}$ & 7.9 & 392 & "-. & 0.3 & $\because .8$ & $1:-0$ \\
\hline & $72-06-22$ & $=$ & 390 & 9.4 & $=$ & -- & 68 & 2.3 & 7.0 & 1.6 \\
\hline & $\begin{array}{l}72-07-18 \\
72-07-20\end{array}$ & $=$ & $\begin{array}{l}360 \\
375\end{array}$ & $\begin{array}{c}12 \\
9.0\end{array}$ & $=$ & $=$ & ${ }^{16}-$ & $4:-5$ & $=$ & $\because$ \\
\hline & $\begin{array}{l}72-10-26 \\
73-04-23\end{array}$ & $=$ & $\begin{array}{l}362 \\
375\end{array}$ & $\begin{array}{l}8.7 \\
8.0\end{array}$ & $=$ & $=-$ & $\begin{array}{l}12 \\
61\end{array}$ & $\begin{array}{l}4.0 \\
4.00\end{array}$ & 9.1 & 1.0 \\
\hline & $73-08-10$ & -- & 380 & 12 & -- & -- & 67 & 3.9 & 1.4 & 8.0 \\
\hline & & -- & $\begin{array}{l}36 \\
37\end{array}$ & $\begin{aligned} 10 \\
8 .\end{aligned}$ & $=$ & $=-$ & $\begin{array}{l}71 \\
63\end{array}$ & $\begin{array}{l}3.6 \\
3.9\end{array}$ & $\begin{array}{l}6.4 \\
7.6\end{array}$ & $\begin{array}{l}9.0 \\
1.0\end{array}$ \\
\hline \multirow[t]{9}{*}{280242082342602} & $70-01-12$ & 23.0 & 45 & 9.0 & 6.0 & 12 & -- & -- & -- & $\cdots$ \\
\hline & $70-02-02$ & 20.0 & 110 & 11 & 6.7 & 57 & $-\infty$ & - & -- & - \\
\hline & $70-09-17$ & 28.0 & 126 & 12 & 7.1 & 316 & $\cdots$ & -- & -- & - \\
\hline & $\begin{array}{l}70-10-26 \\
71-02-26\end{array}$ & $\begin{array}{l}26.0 \\
26.5\end{array}$ & $\begin{array}{r}108 \\
82\end{array}$ & 100 & $\begin{array}{l}6.7 \\
7.7\end{array}$ & $\begin{array}{r}54 \\
226\end{array}$ & $\because$ & $=$ & $=$ & $=-$ \\
\hline & $\begin{array}{l}71-07-27 \\
71-09-22\end{array}$ & $\begin{array}{l}24.0 \\
28.5\end{array}$ & $\begin{array}{r}68 \\
192\end{array}$ & 7.0 & $\begin{array}{l}7.3 \\
6.8\end{array}$ & $\begin{array}{l}256 \\
94\end{array}$ & $=$ & $=$ & $\because$ & $=$ \\
\hline & $71-10-28$ & 27.0 & 70 & 7.0 & 5.7 & 24 & -- & -- & -- & -- \\
\hline & $\begin{array}{l}2-27 \\
3-15\end{array}$ & $\bar{z}$ & $\begin{array}{r}94 \\
370\end{array}$ & 1006 & 6.9 & 40 & $\begin{array}{l}10 \\
13\end{array}$ & $\begin{array}{l}19 \\
11\end{array}$ & $60^{7.6}$ & .6 \\
\hline & & -- & 48 & -- & -- & -- & 13 & 11 & 00 & $\cdot 1$ \\
\hline & & -- & 198 & 12 & -- & -- & 14 & 2.3 & -- & .7 \\
\hline \multirow[t]{13}{*}{280246082343202} & $\begin{array}{l}70-02-06 \\
70-09-17\end{array}$ & 22.0 & 58 & 9.4 & 6.2 & $\overline{170}$ & $\because$ & $=$ & $=$ & $=-$ \\
\hline & $10-22$ & 24.0 & $2 \varepsilon$ & 10 & 7.5 & 143 & 51 & 3.4 & 0.4 & 1.9 \\
\hline & $71-01-14$ & 25.0 & 332 & 10 & 7.8 & $\begin{array}{l}180 \\
192\end{array}$ & $59^{\circ-}$ & 3.8 & 6.2 & 2.1 \\
\hline & $71-02-24$ & 23.5 & 378 & 12 & 7.6 & 232 & -- & -- & $=$ & $=-$ \\
\hline & $71-04-28$ & 24.0 & 37 & 8.0 & $\because-$ & -- & $\because$ & $=$ & $\because$ & - \\
\hline & $\begin{array}{l}71-06-15 \\
71-07-28\end{array}$ & $\begin{array}{l}24.5 \\
26.0\end{array}$ & $\begin{array}{l}354 \\
388\end{array}$ & 16.0 & $\begin{array}{l}7.22 \\
7.5\end{array}$ & $\begin{array}{l}\begin{array}{l}230 \\
260\end{array} \\
200\end{array}$ & $=$ & $=$ & $=$ & $=$ \\
\hline & $71-09-21$ & 29.0 & 363 & 10 & 8.2 & 232 & -- & -- & -- & -- \\
\hline & $\begin{array}{l}71-11-22 \\
71-12-27\end{array}$ & $33 . \overline{5}$ & 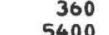 & $1400^{8.3}$ & $7 ?$ & 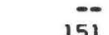 & $=$ & 4.9 & 5.5 & .5 \\
\hline & & 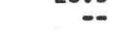 & 23 & $x_{7}$ & 10 & 20 & -0 & 6.2 & 5 & 1.2 \\
\hline & $72-$ & -- & 372 & & -- & -- & & 6.6 & & 1.0 \\
\hline & $\begin{array}{l}72-07-18 \\
73-04-24\end{array}$ & $=$ & $\begin{array}{l}389 \\
36 ?\end{array}$ & 13 & $=$ & $=$ & $\begin{array}{l}84 \\
65\end{array}$ & 5.2 & $\overline{70}$ & 1.6 \\
\hline & & $=$ & 3 & 7.2 & -- & -- & 6 & 4.4 & 6.0 & 1.0 \\
\hline & $\begin{array}{l}73-08-13 \\
73-10-15\end{array}$ & $=$ & $\begin{array}{l}370 \\
374\end{array}$ & $\begin{array}{l}7.2 \\
9.0\end{array}$ & $=$ & $=$ & $\begin{array}{l}66 \\
65\end{array}$ & $\begin{array}{l}4.4 \\
4.2\end{array}$ & $0^{2}+2>3$ & 1.0 \\
\hline \multirow{3}{*}{280246082340301} & & & & & & & & & & \\
\hline & & -- & a & 7.5 & -. & -- & $\begin{array}{l}86 \\
67\end{array}$ & 4.2 & $\begin{array}{l}<.0 \\
5.7\end{array}$ & -8 \\
\hline & & -- & & 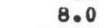 & -- & -0 & & 4 & & .7 \\
\hline \multirow[t]{4}{*}{280243082342501} & $\begin{array}{l}71-11-2 \\
71-12-2\end{array}$ & $=$ & $\begin{array}{l}124 \\
170\end{array}$ & 11 & 8.0 & $1 \overline{120}$ & $15 \ldots$ & 2.6 & 5.0 & $\because 0$ \\
\hline & $72-01-26$ & -- & 108 & 11 & 6.8 & 33 & $\because-$ & $0_{0}$ & $-\overline{0}$ & -- \\
\hline & & $=-$ & $\begin{array}{r}45 \\
183\end{array}$ & $\begin{array}{l}14 \\
6.0\end{array}$ & $=$ & -- & $33^{7.2}$ & $\begin{array}{l}9.8 \\
2.5\end{array}$ & 5.00 & 3.3 \\
\hline & & $=$ & 122 & 10 & $=$ & $=$ & 16 & 2.7 & 8.3 & 4.5 \\
\hline \multirow{5}{*}{280243082342602} & & $=$ & $\begin{array}{c}203 \\
55\end{array}$ & $\begin{array}{l}8.1 \\
5.0\end{array}$ & 6 & -- & 33 & 3.9 & 1.3 & 7.3 \\
\hline & $70-02-03$ & 18.5 & 82 & 5.0 & 6.3 & 39 & -- & -- & $=$ & - \\
\hline & $\begin{array}{l}70-09-17 \\
71-09-22\end{array}$ & 29.0 & $\begin{array}{l}126 \\
383\end{array}$ & 10.9 & 7.2 & 50 & $=$ & -- & $=$ & - \\
\hline & & -- & & & 6.5 & 112 & -- & -- & -- & - \\
\hline & $\begin{array}{l}6-21 \\
40-27\end{array}$ & -- & 96 & 13 & $=$ & $=$ & 8.7 & 2.4 & 5.5 & .0 \\
\hline 10243082342601 & $10-10-20$ & 27.0 & $\begin{array}{l}313 \\
313\end{array}$ & $\because 0$ & 7.8 & 154 & $55^{\circ .0}$ & 3.3 & 5.6 & $1: 2$ \\
\hline & & & & & & & & & & \\
\hline
\end{tabular}


TABLE 6A.--WATER-QUALITY DATA FOR GROUND-WATER SITES AT THE ROCKY CREEK LANDFILL--CONTINUED

SPE-
CIFIC
CONA


Table 6B,--WAter-quality data for ground-water sites at the Rocky CReek LaNDFill

\begin{tabular}{|c|c|c|c|c|c|c|c|c|c|c|}
\hline STATION NUMBER & $\begin{array}{c}\text { DATE } \\
\text { OF } \\
\text { SAMPLE }\end{array}$ & $\begin{array}{l}\text { SOLIUS, } \\
\text { RESIDUE } \\
\text { AI } 180 \\
\text { UEG. C } \\
\text { DIS- } \\
\text { SULVEO } \\
\text { (MG LL) }\end{array}$ & $\begin{array}{c}\text { BICAR- } \\
\text { BONATE } \\
\text { (MG/L } \\
\text { AS } \\
\text { HCO3) }\end{array}$ & $\begin{array}{l}\text { CAR- } \\
\text { BONATE } \\
\text { (MG } \\
\text { AS CO3) }\end{array}$ & $\begin{array}{l}\text { HARD- } \\
\text { NESS } \\
\text { (MG/L } \\
\text { AS } \\
\text { CACO3) }\end{array}$ & $\begin{array}{l}\text { HARO- } \\
\text { NESS, } \\
\text { NONCAR- } \\
\text { BONATE } \\
\text { (MGLL } \\
\text { CACO3) }\end{array}$ & $\begin{array}{l}\text { SULFATE } \\
\text { DIS- } \\
\text { SOLVED } \\
\text { (MG/L } \\
\text { AS SO4) }\end{array}$ & $\begin{array}{l}\text { FLUO- } \\
\text { RIDE, } \\
\text { DIS- } \\
\text { SOLVEO } \\
\text { (MG/L } \\
\text { AS F) }\end{array}$ & $\begin{array}{l}\text { SILICA, } \\
\text { UIS- } \\
\text { SOLVED } \\
\text { (MG/L } \\
\text { AS } \\
\text { SIOZ) }\end{array}$ & $\begin{array}{l}\text { CARBON } \\
\text { OIOXIUEE } \\
\text { OIS- } \\
\text { SOLVED } \\
\text { (MGLL } \\
\text { AS COLI) }\end{array}$ \\
\hline \multirow{2}{*}{280219082342801} & $70-01-29$ & 229 & 230 & 0 & 188 & 0 & .0 & .2 & 15 & 2.3 \\
\hline & $70-10-23$ & 239 & 224 & 0 & 188 & 1 & .3 & .2 & 14 & 11 \\
\hline \multirow[t]{6}{*}{280221082342901} & $70-04-20$ & 230 & 234 & 0 & 103 & 0 & .0 & .2 & 15 & 3.0 \\
\hline & $70-10-23$ & 244 & 224 & 4 & 49 & 0 & .3 & .2 & 14. & $=$ \\
\hline & $72-10-25$ & - & $\cdots$ & -- & ${ }^{4}$ & $\cdots$ & $\cdots$ & $\cdots$ & $\cdots$ & - \\
\hline & $73-02-01$ & -- & -- & -- & 190 & - & -- & $\cdots$ & -- & -- \\
\hline & $73-04-23$ & 230 & 228 & 0 & 190 & 3 & 1.6 & .2 & 12 & 7.3 \\
\hline & $73-10-15$ & -- & -- & -- & 190 & -- & -- & $\cdots$ & -- & -- \\
\hline \multirow[t]{2}{*}{280226082340701} & $70-01-29$ & -- & - & -- & 14 & - & -- & -- & -- & -- \\
\hline & $72-07-17$ & -- & -- & -- & 40 & -- & -- & -- & -- & -- \\
\hline \multirow{19}{*}{280226082342101} & $72-10-2 b$ & -- & -- & -- & 18 & - & -- & -- & -- & -- \\
\hline & $70-01-29$ & -- & -- & -- & 108 & -- & -- & -- & -- & - \\
\hline & $70-04-20$ & -- & -- & -- & 55 & -- & -- & $=$ & $=$ & - \\
\hline & $\begin{array}{l}70-06-25 \\
70-07-15\end{array}$ & $=-$ & $=$ & $=$ & 44 & $=$ & $=$ & $\overline{-}$ & $=$ & $\because$ \\
\hline & 70 & & & & & & & & & $\ldots$ \\
\hline & $70-08-05$ & -- & -- & -- & 51 & -- & $=-$ & -- & -- & $=$ \\
\hline & $\begin{array}{l}70-09-18 \\
70-10-27\end{array}$ & $=$ & $=$ & $=$ & $\begin{array}{l}49 \\
57\end{array}$ & $=$ & $\overline{-}$ & 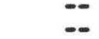 & $\ddot{--}$ & $=$ \\
\hline & $70-12-03$ & - & $=$ & $=$ & 59 & - & $\ldots$ & -- & -- & -- \\
\hline & $71-01-14$ & -- & -- & -- & 66 & - & -- & -- & -- & -- \\
\hline & $71-02-26$ & -- & -- & -- & 81 & $\therefore$ & -- & -- & -- & - \\
\hline & $71-03-31$ & $\cdots$ & -- & -- & 71 & -- & $\cdots$ & $\cdots$ & -- & -- \\
\hline & $11-04-28$ & -- & -- & -- & 128 & -- & $\cdots$ & -- & -- & $=$ \\
\hline & $71-06-15$ & -- & -- & $=$ & 84 & -- & -- & $=$ & -- & $=$ \\
\hline & $71-07-28$ & -- & -- & -- & 90 & -- & -- & $\cdots$ & -- & - \\
\hline & $71-09-21$ & -- & -- & -- & 190 & - & -- & -- & -- & - \\
\hline & $71-10-29$ & - & -- & -- & 73 & - & -- & -- & -- & -- \\
\hline & $72-03-16$ & -- & - & -- & 75 & - & - & -- & -- & $=$ \\
\hline & $72-07-17$ & -- & -- & -- & 90 & $\cdots$ & -- & -- & -- & - \\
\hline & $72-10-25$ & -- & $\cdots$ & -- & 92 & $\cdots$ & -- & -- & -- & -- \\
\hline 280226082342502 & $70-01-29$ & -- & - & -- & 35 & -- & -- & - & -- & -- \\
\hline & $70-04-20$ & -- & -- & -- & 86 & -- & -- & -- & -- & -- \\
\hline & $70-06-25$ & -- & - & -- & 40 & -- & -- & -- & $\cdots$ & -- \\
\hline & $70-07-15$ & -- & -- & -- & 48 & - & -- & -- & -- & -- \\
\hline & $70-08-05$ & $\cdots$ & -- & -- & 42 & -- & -- & -- & -- & -- \\
\hline & $70-10-26$ & -- & - & -- & 52 & -- & -- & -- & -- & -- \\
\hline & $70-12-03$ & $\cdots$ & -- & -- & 60 & -- & $\cdots$ & -- & -- & $=$ \\
\hline & $71-01-14$ & $=$ & -- & -- & 44 & $=$ & $=$ & -- & $=$ & $=$ \\
\hline & $71-02-26$ & -- & -- & -- & 47 & -- & $=$ & $=$ & $=$ & $=$ \\
\hline & $71-03-30$ & -- & -- & -- & 50 & -- & -- & & -- & - \\
\hline & $71-04-28$ & -- & $-\infty$ & -- & 37 & -- & -- & - & -- & -- \\
\hline & $71-07-27$ & -- & - & -- & 57 & - & -- & -- & -- & $\cdots$ \\
\hline & $71-09-21$ & -- & -- & -- & 50 & -- & -- & -- & -- & -- \\
\hline & $71-10-29$ & -- & $\cdots$ & -- & 77 & -- & -- & $=$ & -- & -- \\
\hline & $71-11-22$ & -- & -- & -- & 94 & -- & -- & -- & -- & -- \\
\hline & $72-01-27$ & -- & -- & -- & 76 & -- & -. & -- & -- & -- \\
\hline & $72-01-27$ & -- & 116 & 0 & -- & - & -- & -- & -- & 37 \\
\hline & $72-07-17$ & -- & - & -- & 370 & - & $\cdots$ & -- & -- & -- \\
\hline & $72-10-25$ & -- & -- & -- & 120 & -- & -- & -- & -- & -- \\
\hline & $73-02-01$ & $\cdots$ & -- & -- & 64 & -- & -- & -- & $\cdots$ & - \\
\hline & $73-03-16$ & -- & - & -- & 150 & -. & -- & -- & -- & -- \\
\hline & $73-04-26$ & -- & - & -- & 92 & - & -- & - & -- & -- \\
\hline 280226082342501 & $70-01-30$ & -- & -- & -- & 142 & -- & -- & $\cdots$ & -- & - \\
\hline & $\begin{array}{l}70-04-20 \\
70-06-25\end{array}$ & $\ddot{m}$ - +4 & $=$ & $=$ & 196 & $=$ & $\overline{-}$ & $=$ & $=$ & $\overline{-}+$ \\
\hline & $70-06-25$ & $\cdots$ & -- & - & 193 & $\cdots$ & $\cdots$ & $\cdots$ & -- & -- \\
\hline & $70-07-15$ & -- & - & -- & 118 & -- & -- & -- & -- & -- \\
\hline & $70-08-05$ & -- & -- & -- & 190 & - & -- & $\cdots$ & -- & -- \\
\hline & $70-10-26$ & -- & -- & -- & 186 & - & -- & -- & -- & -- \\
\hline & $70-12-03$ & -- & -- & -- & 181 & -- & $\cdots$ & -- & -- & -- \\
\hline & $71-01-14$ & -- & -- & -- & 194 & - & -- & -- & -- & -- \\
\hline & $71-02-26$ & -- & - & -- & 117 & - & - & -- & -- & - \\
\hline & $71-03-31$ & -- & -- & -- & 192 & -- & -- & -- & -- & -- \\
\hline & $71-04-28$ & -- & -- & -- & 201 & -- & -- & $\cdots$ & -- & -- \\
\hline & $71-06-15$ & -- & -- & -- & 178 & -- & -- & -- & -- & - \\
\hline & $71-07-27$ & -- & -- & -- & 191 & -- & -- & -- & -- & -- \\
\hline & $71-11-22$ & -- & -- & & 150 & -- & & & -- & - \\
\hline & $72-07-18$ & -- & -- & -- & 220 & -- & - & - & -- & $\cdots$ \\
\hline & $72-10-25$ & -- & - & -- & 200 & -- & -- & $\cdots$ & -- & -- \\
\hline & $73-04-26$ & -- & $-\infty$ & -- & 180 & $\cdots$ & -- & -- & $-\infty$ & -- \\
\hline & $73-08-09$ & -- & -- & -- & 190 & -- & $-\infty$ & -- & -- & -- \\
\hline & $73-12-19$ & -- & -- & -- & 180 & -- & - & - & -- & - \\
\hline 280226082342902 & $70-04-20$ & - & $\cdots$ & - & 73 & -- & 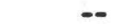 & -- & - & -- \\
\hline & $70-06-25$ & -- & - & -- & 175 & - & -- & -- & -- & -- \\
\hline 280226082341202 & $73-04-24$ & 63 & 6 & 0 & 13 & 8 & 14 & .2 & 9.2 & 61 \\
\hline 280226082341201 & $73-04-25$ & -- & -- & -- & 210 & - & -- & 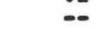 & -- & - \\
\hline 280226082342504 & $71-12-28$ & -- & - & -- & 190 & -- & - & -- & -- & - \\
\hline & $72-01-26$ & -- & -- & -- & 190 & -- & $-\infty$ & -- & -- & 12 \\
\hline & $72-03-16$ & -- & -- & -- & 190 & -- & -- & $=$ & $=$ & - \\
\hline & $\begin{array}{r}72-07-19 \\
72-10-25\end{array}$ & $=$ & $=$ & $=$ & 270 & $=$ & $=$ & $=$ & $=$ & $=$ \\
\hline & $72-10-25$ & - & 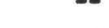 & - & 190 & - & - & - & & \\
\hline
\end{tabular}


Table 6B,--Water-quality data for ground-water sites at the Rocky CReek landfill--Continued

\begin{tabular}{|c|c|c|c|c|c|c|c|c|c|c|}
\hline STATION NUMBER & $\begin{array}{c}\text { DATE } \\
\text { OF } \\
\text { SAMPLE }\end{array}$ & $\begin{array}{l}\text { SOL IOS, } \\
\text { RESIDUE } \\
\text { AT I } 180 \\
\text { DEG. C } \\
\text { DIS- } \\
\text { SOLVED } \\
\text { (MG/L) }\end{array}$ & $\begin{array}{c}\text { BICAR- } \\
\text { BONATE } \\
\text { (MG/L } \\
\text { AS } \\
\text { HCO3) }\end{array}$ & $\begin{array}{l}\text { CAR- } \\
\text { BONATE } \\
\text { (MGLL } \\
\text { AS CO3) }\end{array}$ & $\begin{array}{l}\text { HARD- } \\
\text { NESS } \\
\text { (MG/L } \\
\text { AS } \\
\text { CACO3) }\end{array}$ & $\begin{array}{l}\text { HARD- } \\
\text { NESS, } \\
\text { NONCAR- } \\
\text { BONATE } \\
\text { (MG/L } \\
\text { CACO3) }\end{array}$ & $\begin{array}{l}\text { SULFATE } \\
\text { DIS- } \\
\text { SOLVEO } \\
\text { (MG/L } \\
\text { AS SO4) }\end{array}$ & $\begin{array}{l}\text { FLUO- } \\
\text { RIDE, } \\
\text { OIS- } \\
\text { SOLVED } \\
\text { (MG/L } \\
\text { AS F) }\end{array}$ & $\begin{array}{l}\text { SILICA, } \\
\text { DIS- } \\
\text { SOLVED } \\
\text { (MG/L } \\
\text { AS } \\
\text { SIO2) }\end{array}$ & $\begin{array}{l}\text { CAKBON } \\
\text { OIOXIDE } \\
\text { OIS- } \\
\text { SOLVED } \\
\text { (MG/L } \\
\text { AS COZ) }\end{array}$ \\
\hline \multirow[t]{4}{*}{280226082342504} & $73-04-23$ & -- & $\cdots$ & -- & 180 & -- & -- & -- & -- & -- \\
\hline & $73-08-09$ & $\cdots$ & -- & -- & 190 & -- & -- & $\cdots$ & - & -- \\
\hline & $73-10-15$ & -- & -- & -- & 190 & -- & $\cdots$ & $\cdots$ & -- & -- \\
\hline & $73-12-19$ & -- & -- & -- & 180 & -- & $\cdots$ & -- & $\cdots$ & $=$ \\
\hline \multirow[t]{28}{*}{280227082343001} & $70-01-29$ & 226 & 230 & 0 & 188 & 0 & .0 & .2 & 15 & 3.7 \\
\hline & $70-04-20$ & -- & -- & -- & 187 & -- & -- & -- & - & -- \\
\hline & $\begin{array}{l}70-06-02 \\
70-06-25\end{array}$ & 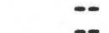 & $\because$ & $=$ & 131 & $\because$ & $\because$ & 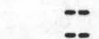 & $=$ & $=$ \\
\hline & $70-07-14$ & $=$ & $=$ & -- & $\begin{array}{r}178 \\
89\end{array}$ & 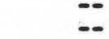 & $=$ & $=$ & $\Longrightarrow$ & 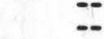 \\
\hline & $70-08-04$ & -- & - & -- & 160 & - & - & - & -- & -- \\
\hline & $70-09-18$ & -- & - & -- & 186 & -- & -- & -- & -- & -- \\
\hline & $70-10-22$ & 220 & 224 & 4 & 190 & 1 & .7 & .3 & 14 & 15 \\
\hline & $70-12-03$ & -- & -- & -- & 179 & -- & - & -- & -- & -- \\
\hline & $71-01-14$ & -- & $-\infty$ & -- & 193 & -- & $\cdots$ & -- & $-\infty$ & -- \\
\hline & $71-02-24$ & -- & - & -- & 157 & -- & -- & -- & - & - \\
\hline & $71-03-31$ & -- & - & -- & 190 & - & - & - & - & -- \\
\hline & $71-04-28$ & -- & -- & -- & 140 & -- & -- & $=-$ & -- & -- \\
\hline & $71-06-15$ & -- & -- & -- & 190 & -- & $\cdots$ & -- & -- & $\cdots$ \\
\hline & $71-07-28$ & -- & -- & -- & 188 & -- & -- & -- & -- & -- \\
\hline & $71-09-22$ & -- & -- & -- & 200 & -- & -- & -- & $\cdots$ & $\cdots$ \\
\hline & $71-10-28$ & -- & -- & -- & 190 & -- & -- & -- & -- & -- \\
\hline & $71-11-22$ & -- & -- & -- & 150 & -- & -- & -- & - & $\cdots$ \\
\hline & $\begin{array}{l}71-12-22 \\
71-12-27\end{array}$ & $=$ & $=$ & $=$ & 150 & $=$ & $\overline{-}$ & $=$ & $=$ & $=-$ \\
\hline & $\begin{array}{l}71-12-27 \\
72-03-17\end{array}$ & $=$ & $=$ & $=$ & 190 & $=$ & $=$ & $=$ & $=$ & 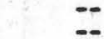 \\
\hline & 1) & & & & $4 \pi 0$ & 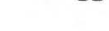 & & & & \\
\hline & $72-06-20$ & -- & -- & -- & 190 & -- & $\cdots$ & $\cdots$ & $\cdots$ & -. \\
\hline & $72-07-18$ & -- & -- & -- & 230 & $\cdots$ & $\cdots$ & -- & - & - \\
\hline & $72-10-25$ & $\cdots$ & $\cdots$ & -- & 180 & $\cdots$ & $\cdots$ & -- & $\cdots$ & $\cdots$ \\
\hline & $73-02-01$ & -- & -- & -- & 190 & -- & - & -- & - & -- \\
\hline & $73-04-23$ & -- & -- & -- & 180 & - & - & -- & -- & $\cdots$ \\
\hline & $73-08-09$ & -- & -- & -- & 190 & -- & - & -- & - & - \\
\hline & $73-10-15$ & $\cdots$ & -- & -- & 190 & $\cdots$ & -- & $\cdots$ & $\cdots$ & $\cdots$ \\
\hline & 73-12-19 & $=$ & $=$ & -- & 190 & -- & -- & $=$ & $=$ & $=$ \\
\hline \multirow[t]{3}{*}{280228082335901} & $72-07-17$ & -- & - & -- & 64 & -- & -- & -- & - & -- \\
\hline & $10-100<0$ & - & - & $\cdots$ & 15 & $\cdots$ & -0 & 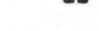 & 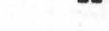 & $-\infty$ \\
\hline & $73-02-01$ & -- & -- & -- & 58 & -- & - & -- & $-\infty$ & -- \\
\hline \multirow{3}{*}{280228082335902} & $\begin{array}{l}13-04-26 \\
73-07-17\end{array}$ & & $\overline{-}$ & -- & 31 & -- & $\cdots$ & $\cdots$ & $=$ & 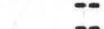 \\
\hline & $72-10-26$ & $\ldots$ & $\ldots$ & $=$ & 170 & $\ldots$ & $\ldots$ & $=$ & $\ldots$ & -- \\
\hline & $73-02-01$ & -- & -- & -- & 180 & -- & - & -- & - & - \\
\hline \multirow{4}{*}{280228082341201} & $73-04-26$ & - & -- & -- & 180 & -- & - & - & $\cdots$ & - \\
\hline & $73-08-13$ & $\cdots$ & - & -- & 31 & - & $\cdots$ & $\cdots$ & -- & $\ddot{-\infty}+1+x$ \\
\hline & $73-10-17$ & $=$ & -- & -- & 13 & $=$ & $=$ & $=$ & 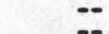 & $=$ \\
\hline & $73-12-20$ & -- & 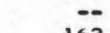 & $-\overline{0}$ & 29 & 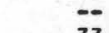 & $-\infty$ & $-\overline{-}+2+3$ & - & $=$ \\
\hline 280228082342601 & $70-01-30$ & 316 & 162 & 0 & 210 & 77 & 74 & .3 & 14 & 5.2 \\
\hline & $70-04-20$ & $-\infty$ & -- & -- & 207 & -- & - & -- & $\cdots$ & -- \\
\hline & $\begin{array}{l}70-06-25 \\
70-07-15\end{array}$ & $=$ & $=$ & $=$ & 192 & $=$ & $\overline{-}$ & $=$ & $=$ & $=-$ \\
\hline & $\begin{array}{l}70-07-15 \\
70-08-05\end{array}$ & $\ldots$ & - & $=$ & $\begin{array}{r}154 \\
91\end{array}$ & $=$ & - & - & $=$ & - \\
\hline & $70-09-18$ & -- & -- & -- & 130 & - & - & $\cdots$ & -- & $\cdots$ \\
\hline & $70-10-23$ & 257 & 240 & 0 & 210 & 9 & 11 & .3 & 13 & $\cdots$ \\
\hline & $70-12-04$ & -- & -- & -- & 168 & $\cdots$ & $\cdots$ & -- & $\cdots$ & - \\
\hline & $71-01-13$ & -- & -- & -- & 190 & -- & -- & $=$ & $=$ & $=$ \\
\hline & $71-02-26$ & $\cdots$ & $=$ & $=$ & 37 & $=$ & $=$ & $\cdots$ & $=$ & $=$ \\
\hline & $71-03-30$ & -- & $\cdots$ & -- & 217 & $-\infty$ & - & $\cdots$ & -- & $\cdots$ \\
\hline & $71-04-28$ & -- & -- & -- & 203 & -- & -- & -- & -- & $\because$ \\
\hline & $71-06-14$ & -- & -- & -- & 200 & $\cdots$ & - & -- & - & - \\
\hline & $71-07-28$ & 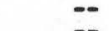 & $=$ & $=$ & 191 & $\because$ & $\because$ & $\because$ & $=$ & $=-$ \\
\hline & $71-09-22$ & $\overline{-}$ & 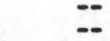 & $=$ & 190 & $\Longrightarrow$ & $\Longrightarrow$ & $\because$ & 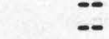 & $=$ \\
\hline & & - & $-\infty$ & -- & & $\cdots$ & -- & $\ldots$ & -- & -- \\
\hline & $\begin{array}{l}1-11-22 \\
71-12-28\end{array}$ & $\ldots$ & $=$ & $=$ & 200 & $=$ & - & - & -. & $\ldots$ \\
\hline & $72-07-19$ & $\cdots$ & -- & - & 270 & $\cdots$ & $\cdots$ & $\cdots$ & -- & $=$ \\
\hline & $72-10-25$ & $\cdots$ & $=$ & $=$ & 190 & $=$ & $\overline{-\infty}$ & $=$ & $=$ & $\because$ \\
\hline & $73-02-01$ & -- & -- & -- & 230 & $-\infty$ & - & - & -- & -- \\
\hline & $73-04-23$ & -- & $\cdots$ & -- & 190 & 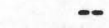 & -- & -- & - & - \\
\hline & $73-08-09$ & $\cdots$ & $\cdots$ & $\cdots$ & 180 & $-\infty$ & $-\infty$ & -- & -- & - \\
\hline & & $=$ & $=$ & $=$ & 180 & $=$ & $=$ & $=$ & $=$ & $=$ \\
\hline & & 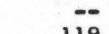 & 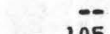 & $=$ & 190 & $\because$ & $\overline{5}$ & - & 5 & $27^{--}$ \\
\hline 280228082342602 & $70-01-30$ & 119 & 105 & 0 & 87 & 1 & 5.6 & .1 & 5.1 & 27 \\
\hline & $70-04-20$ & -- & -- & $\cdots$ & 113 & - & $\cdots$ & $\cdots$ & $\cdots$ & - \\
\hline & $70-06-25$ & -- & - & -- & 67 & $\cdots$ & $\cdots$ & - & -- & - \\
\hline & $70-07-15$ & -- & $\cdots$ & $=$ & 65 & $-\infty$ & $-\infty$ & $=$ & $\cdots$ & - \\
\hline & $70-08-05$ & -- & - & -- & 73 & $=$ & $=$ & -- & 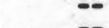 & - \\
\hline & $70-09-18$ & $\cdots$ & $\cdots$ & $\cdots$ & 64 & $-\infty$ & -- & -- & -- & - \\
\hline & $71-02-26$ & - & - & - & 81 & - & $\cdots$ & -- & $-\infty$ & -- \\
\hline & $71-09-22$ & - & - & -- & 120 & - & $\cdots$ & $\cdots$ & $-\infty$ & -- \\
\hline & $71-10-$ & $=$ & $=$ & $\ddot{-}$ & 160 & $-\infty$ & $=$ & $=$ & $=$ & 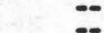 \\
\hline & $73-c$ & $m$ & $-\infty$ & - & 160 & - & - & $\cdots$ & $=$ & - \\
\hline 28022 & $70-01-30$ & 188 & 184 & 0 & 135 & 0 & 9.6 & $\cdot 2$ & 6.3 & 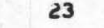 \\
\hline
\end{tabular}




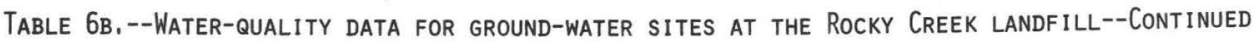

\begin{tabular}{|c|c|c|c|c|c|c|c|c|c|c|c|}
\hline & & & $\begin{array}{l}\text { OLIUS, } \\
\text { ESIDUEE }\end{array}$ & & & & HARL & & & & \\
\hline & & & $\begin{array}{ll}180 \\
6.0\end{array}$ & $\begin{array}{l}\text { BICAR- } \\
\text { BONATE }\end{array}$ & & $\begin{array}{l}\text { HARD- } \\
\text { NESS }\end{array}$ & $\begin{array}{l}\text { NESS, } \\
\text { NONCAR- }\end{array}$ & $\begin{array}{l}\text { SUL } \\
\text { DI }\end{array}$ & & & DI \\
\hline ATION & 2 & $\begin{array}{c}\text { OF } \\
\text { SAMPI }\end{array}$ & $\begin{array}{l}\text { UIS- } \\
\text { SOLVED } \\
\text { (MG/L) }\end{array}$ & $\begin{array}{l}\text { (MG/L } \\
\text { AS } \\
H C O 3)\end{array}$ & $\begin{array}{l}\text { BONATE } \\
\text { (MG/L } \\
\text { AS CO3) }\end{array}$ & $\begin{array}{l}\text { (MG/L } \\
\text { AS } \\
\text { CACO3) }\end{array}$ & $\begin{array}{l}\text { BONATE } \\
\text { (MG/L } \\
\text { CACU3) }\end{array}$ & $\begin{array}{l}\text { SOLVED } \\
\text { (MG/L } \\
\text { AS SO4) }\end{array}$ & $\begin{array}{l}\text { SOLVED } \\
\text { (MG/L } \\
\text { AS F) }\end{array}$ & $\begin{array}{l}\text { IMG/L } \\
\text { AS } \\
\text { SIOZ) }\end{array}$ & $\begin{array}{l}\text { (MG/L } \\
S \text { COZZ) }\end{array}$ \\
\hline
\end{tabular}

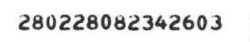

$\begin{array}{lll}-- & -- & = \\ -- & = & = \\ -- & = & =\end{array}$

\section{4}

154
107
132
176
166

$$
\overline{--}
$$$$
70-07-15
$$$$
70-08-05
$$

$70-10-23$

$70-12-04$

$71-01-13$

$71-02-26$
$71-03-30$

210

220

--

$$
0
$$$$
\begin{aligned}
& 176 \\
& 166
\end{aligned}
$$$$
=
$$$$
\because:-
$$$$
\begin{array}{r}
173 \\
136 \\
181 \\
59 \\
179
\end{array}
$$

$71-04-26$

$71-06-14$

$71-07-28$
$71-09-22$

$71-10-29$

$71-11-22$

$71-12-28$

$72-03-16$

$72-07-19$
$72-10-26$

$73-02-01$

$73-04-23$

280228082342701

$71-10-28$

280231082343603

$72-03-16$
$70-01-13$

$70-02-04$

$70-04-23$

$70-06-15$

$70-07-14$

$70-08-04$

$70-09-17$

$70-10-22$

$70-12-03$

$71-01-14$

$71-02-24$

71-03-31

$71-04-28$

$71-06-15$

$71-07-28$
$71-09-21$

$71-10-28$

$71-11-22$

$71-12-27$

72-03-16

73-04-24

73-08-09

73-10-15

$73-12-20$

280231082343602

$70-04-23$

70-06-15

$70-06-25$

$70-07-14$

$70-08-04$

$70-09-17$

$70-12-03$

$71-01-14$

$71-02-24$

$71-03-31$
$71-04-28$

$71-06-15$

$71-07-28$

$71-09-21$

$71-10-28$

$71-10-28$
$71-11-22$

$71-12-27$

$72-01-26$

$72-03-16$

$72-07-18$

$72-10-25$

$73-02-01$

$73-04-24$

73-08-09

$73-10-15$

$73-12-19$

280231082343601

$70-01-14$

$70=02-04$

$70-04-23$
$70-06-15$

$70-06-15$
$70-06-25$

$\begin{array}{llll}-- & = & -- & 184 \\ =- & = & = & 110 \\ =- & = & -- & 166 \\ -- & -- & -- & 180\end{array}$

$\begin{array}{llll}- & - & -- & 180 \\ - & -- & - & 160\end{array}$

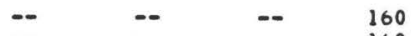

$\begin{array}{llll}-- & -- & = & 140 \\ -- & = & = & 180 \\ - & -- & - & 220\end{array}$

-

$\begin{array}{ll}- & = \\ \therefore & 2160 \\ -- & -\end{array}$

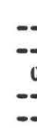

160

160
160
360

360
530

86

86

64

$--$

0
--
--

32
22
16
17
16

72

$\overline{-0}$

$-$

$-\overline{4}$

$-\overline{-} \quad-\overline{0}$

$\begin{array}{lll}-- & -- \\ - & -\end{array}$

$=\square$

$\begin{array}{lll}-- & - \\ -- & -\end{array}$

$\begin{array}{ll}-\infty & - \\ -- & --\end{array}$

$\therefore$

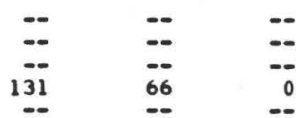

5
19
12
34
10

$$
--
$$

$\begin{array}{ll}0 & 4.4 \\ -- & =- \\ -- & =-\end{array}$

$\begin{array}{llll}-2 & -1 & - & -\end{array}$

$.3 \quad 8.0$

$\begin{array}{lll}- & = & - \\ - & = & =\end{array}$

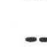

$=--$

$\because$$$
\overline{--}
$$$$
=
$$$$
\begin{array}{ll}
-- & = \\
-- & = \\
-- & =
\end{array}
$$

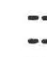$$
\because
$$$$
\text { -- }
$$$$
-
$$$$
\text { -- }
$$$$
\because
$$$$
\overline{--}
$$

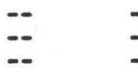$$
\text { ב }
$$$$
-\overline{0}
$$$$
8
$$$$
\cdots
$$$$
\because
$$$$
-
$$$$
\begin{array}{ll}
-- & -- \\
8.6 & = \\
-- & =
\end{array}
$$$$
\begin{array}{cc}
0 & 4.0 \\
-- & -- \\
10 & 9.6 \\
-- & --
\end{array}
$$$$
\text { -- }
$$$$
\begin{array}{rr}
3.8 & 10 \\
3 .-2 & = \\
-- & =- \\
- & -1
\end{array}
$$$$
\text { -- }
$$$$
\begin{aligned}
& - \\
& 9 \\
& -- \\
& --
\end{aligned}
$$$$
30
$$$$
30
$$$$
\begin{array}{cc}
-5 & = \\
3.8 & = \\
-- & =
\end{array}
$$$$
\begin{array}{ll}
-- & 14 \\
-- & 16
\end{array}
$$$$
\begin{array}{ll}
= & 16 \\
= & 10 \\
-- & 12
\end{array}
$$

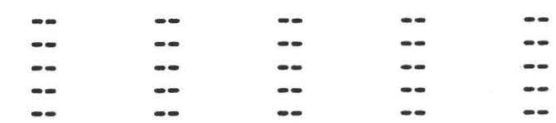$$
\text { -- } 18
$$$$
68
$$$$
44
$$$$
\begin{array}{r}
44 \\
63 \\
120
\end{array}
$$$$
-
$$$$
\ddot{--}
$$$$
-
$$$$
-
$$$$
\overline{-}
$$$$
\because
$$$$
\begin{aligned}
& 22 \\
& 15 \\
& 23 \\
& 68 \\
& 82
\end{aligned}
$$

$$
0
$$

$$
\begin{aligned}
& 68 \\
& 66 \\
& 68 \\
& 47
\end{aligned}
$$$$
\begin{aligned}
& 68 \\
& 66
\end{aligned}
$$$$
\because 1
$$$$
\begin{array}{lll}
- & - & - \\
- & - & =
\end{array}
$$$$
48
$$$$
\begin{array}{lll}
-- & - \\
- & -
\end{array}
$$$$
\begin{array}{lll}
- & - \\
- & - \\
- & -
\end{array}
$$$$
\begin{array}{ll}
-- & 54 \\
- & 48 \\
- & 34 \\
- & 47 \\
& 45
\end{array}
$$$$
\begin{array}{lll}
-- & -1 \\
-\infty & -2
\end{array}
$$$$
\begin{array}{ll}
-- & - \\
-\therefore & = \\
-- & -
\end{array}
$$$$
\begin{array}{ll}
-- & 54 \\
- & 44
\end{array}
$$$$
\begin{array}{rr}
= & 44 \\
= & 78 \\
= & 142 \\
- & 150
\end{array}
$$$$
\begin{array}{ll}
-- & - \\
- & 56 \\
- & --
\end{array}
$$$$
\begin{array}{ll}
-0 & 93 \\
0 & 62
\end{array}
$$$$
\begin{array}{r}
93 \\
62 \\
220 \\
41
\end{array}
$$$$
\begin{array}{llll}
-- & - & - & 4 \\
- & - & - & 44
\end{array}
$$$$
\begin{array}{llll}
-- & -- & - & 20 \\
-- & -- & - & 29
\end{array}
$$$$
\begin{array}{ll}
- & = \\
- & =
\end{array}
$$$$
=
$$$$
\begin{aligned}
& 20 \\
& 29 \\
& 29 \\
& 17
\end{aligned}
$$$$
\begin{array}{ll}
=- & 228 \\
=- & \\
222 & 198
\end{array}
$$

228
198
--

-
-0
$\because-$

126
192
190
98
162 
Table 6B,--Water-quality data for ground-water sites at the Rocky CReek LANDfill--Continued

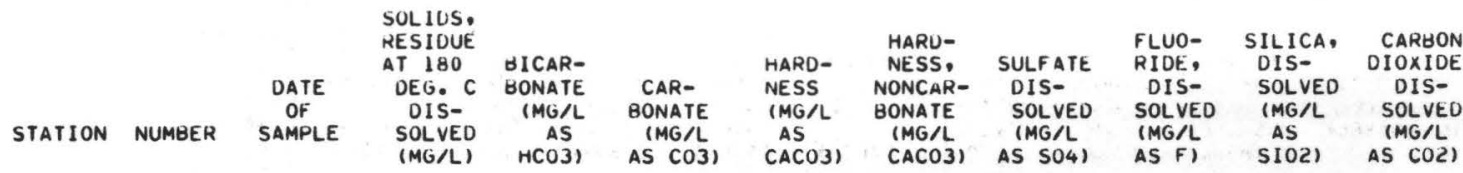

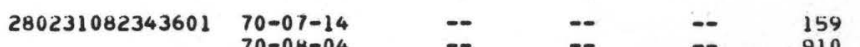

$70-08-04$

$70-09-17$

$70-10-22$

$\begin{array}{llll}z & = & = & 1 \\ z & = & = & 9 \\ z & = & = & 1 \\ - & = & -- & 1\end{array}$

159
910
168
192
151

$-\overline{0} \quad--$

$\because$

$\begin{array}{lll}- & = & =\end{array}$

$71-01-14$

$71-02-24$

$71-03-31$

$71-06-15$

196

114

$71-09-21$

$71-10-28$

$71-11-22$
$71-12-27$

72-03-16

72-07-18

$72-10-25$

73-02-01

$73-04-24$
$73-08-09$

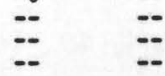

$\bar{z}$

$\ddot{--} \quad=$

$\overline{-0}$

$\begin{array}{lllll}- & - & - & & 188 \\ - & = & - & 152\end{array}$

152
190
260

260
380

-

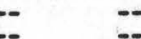

73-10-15

$73-12-19$

$70-01-30$

280231082342602

$70-04-20$

$70-07-15$

$\ddot{--}$

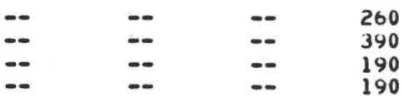

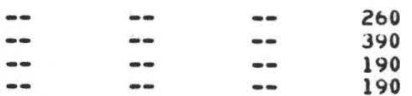

190

ב-

$\because$

$\because$

$\begin{array}{llll}-- & -- & -- & 190 \\ -- & -- & - & 190 \\ 98 & 96 & -- & 58 \\ -- & -- & -- & 95\end{array}$

$\overline{0} \quad \overline{0}$

70-08-04

70-09-18

$70-10-23$

$70-12-04$

-

$\because$

$--$

$71-02-26$

$71-03-30$

$71-04-28$

$71-04-28$
$71-06-14$

71-07-27

$140 \quad 132$

$\begin{array}{cr}-- & 44 \\ -- & 95 \\ 0 & 96 \\ -- & 98 \\ -- & 76\end{array}$

$\begin{array}{ll}-- & = \\ - & - \\ - & 9.5 \\ -- & --\end{array}$

$\begin{array}{ll}-- & - \\ -- & - \\ - & -\end{array}$

71-09-22

$71-12-28$
$71-10-29$

$71-12-28$
$72-04-27$

280231082342601

$70-01-30$

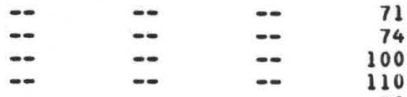

70-04-20

$71-02-26$

$280233082340702 \quad \begin{array}{r}73-02-01 \\ 73-08-13 \\ 73-10-16\end{array}$

$\begin{array}{lllll}73-10-16 & - & - & - & 200\end{array}$

$\begin{array}{ll}-- & = \\ -8 & = \\ 8 & 10\end{array}$

$\begin{array}{ll}- & = \\ z & = \\ -- & =\end{array}$

$=$

$280233082340701 \quad \begin{array}{r}73-12-19 \\ 72-01-27\end{array}$

$72-01-27$
$72-07-17$
$73-04-25$

$73-04-25$
$73-08-13$

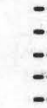

$=--$

$\overline{-}$

73-10-16

$73-12-20$

$70-01-19$

$70-01-19$
$70-02-04$

$70-04-23$

22

$-$

280236082343902

70-06-25

$70-07-14$

70-08-04

$70-09-17$

$70-10-22$

$71-01-14$
$71-02-24$

$71-02-24$
$71-03-31$

$71-03-31$
$71-04-28$

$71-06-14$

$71-07-28$

72-03-16

$72-07-18$
$72-10-25$

75-10-25

$73-04-23$

$70-08-05$

$70-09-18$

$70-10-26$
$71-01-13$

190
76
270
35

35

$\therefore$

-

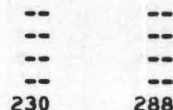

$\therefore$

$\overline{-}$

$\begin{array}{lll}z & = & = \\ z & = & = \\ z & = & =\end{array}$

77
190

68
188

114

$=$

$\begin{array}{llll}- & = & = & 190 \\ = & = & = & 183 \\ = & = & = & 200 \\ = & = & = & 240\end{array}$

28023308234230

$71-02-24$

$71-03-31$

$71-04-28$

$71-06-14$
$71-07-27$

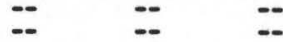


Table 6b. --Water-quality data for ground-water sites at the Rocky Creek landfill--Continued

\begin{tabular}{|c|c|c|c|c|c|c|c|c|c|c|}
\hline STATION NUMBER & $\begin{array}{c}\text { DATE } \\
\text { OF } \\
\text { SAMPLE }\end{array}$ & $\begin{array}{l}\text { SUL IOS, } \\
\text { RESIOUE } \\
\text { AT IBO } \\
\text { DEG. C } \\
\text { DIS- } \\
\text { SOLVED } \\
\text { (MG/L) }\end{array}$ & $\begin{array}{l}\text { OICAR- } \\
\text { BONATE } \\
\text { (MGAL } \\
\text { AS } \\
\text { HCO3) }\end{array}$ & $\begin{array}{l}\text { CAR- } \\
\text { BONATE } \\
\text { (MG/L } \\
\text { AS CO3) }\end{array}$ & $\begin{array}{l}\text { HARO- } \\
\text { NESS } \\
\text { (MG/L } \\
\text { AS } \\
\text { CACO3) }\end{array}$ & $\begin{array}{l}\text { HARD- } \\
\text { NESS } \\
\text { NONCAR- } \\
\text { BONATE } \\
\text { (MG/L } \\
\text { CACO3) }\end{array}$ & $\begin{array}{l}\text { SULFATE } \\
\text { DIS- } \\
\text { SOLVED } \\
\text { (MG/L } \\
\text { AS SO4) }\end{array}$ & $\begin{array}{l}\text { FLUO- } \\
\text { RIDE. } \\
\text { OIS- } \\
\text { SOLVED } \\
\text { (MGAL } \\
\text { AS F) }\end{array}$ & $\begin{array}{l}\text { SILICA, } \\
\text { DIS- } \\
\text { SOLVED } \\
\text { (MGAL } \\
\text { AS } \\
\text { SI02) }\end{array}$ & $\begin{array}{c}\text { CARBON } \\
\text { OIOXIDE } \\
\text { OIS- } \\
\text { SOLVEO } \\
\text { (MG/L } \\
\text { AS COZ) }\end{array}$ \\
\hline \multirow[t]{8}{*}{280233082342302} & $70-02-05$ & 60 & 18 & 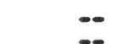 & 25 & 10 & 11 & 1 & 5.3 & 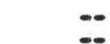 \\
\hline & $\begin{array}{l}70-08-05 \\
70-09-18\end{array}$ & $=$ & $=$ & 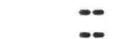 & 7 & 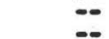 & $\because$ & $=$ & $=$ & $\because$ \\
\hline & $70-10-26$ & $=$ & $\ldots$ & $\ldots$ & 31 & - & $\ldots$ & $\ldots$ & $\ldots$ & 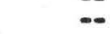 \\
\hline & $70-12-03$ & -- & $=$ & $\cdots$ & 34 & - & -- & - & $-\infty$ & $\infty$ \\
\hline & $71-01-13$ & 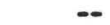 & $\ldots$ & - & 16 & $\infty$ & - & - & - & $\infty$ \\
\hline & $71-02-24$ & $\cdots$ & -- & -- & 13 & - & $\cdots$ & $\cdots$ & $\cdots$ & $\cdots$ \\
\hline & $71-03-31$ & $\cdots$ & $\cdots$ & -- & 10 & -- & $\cdots$ & $=$ & - & $=$ \\
\hline & $71-06-14$ & $\ldots$ & $\ldots$ & $\because$ & 25 & $\because$ & $\because$ & $=$ & $=$ & $=$ \\
\hline \multirow{5}{*}{280233082342603} & $71-07-27$ & 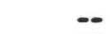 & 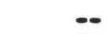 & - & 17 & $\ldots$ & -- & - & - & $-\infty$ \\
\hline & $70-01-30$ & 49 & 21 & -- & 13 & 0 & - & $\cdots$ & $\cdots$ & 13 \\
\hline & $71-09-22$ & -- & $-\infty$ & - & 33 & -- & 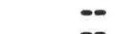 & $\cdots$ & $=$ & $=$ \\
\hline & $\begin{array}{l}71-10-29 \\
71-12-28\end{array}$ & $=$ & $\because$ & $=$ & $\begin{array}{r}25 \\
140\end{array}$ & 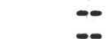 & $=$ & 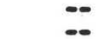 & $\because$ & $=$ \\
\hline & & & & & & & & & & \\
\hline \multirow{15}{*}{280233082342602} & $73-04-27$ & - & $-\infty$ & -- & 23 & $\cdots$ & $\cdots$ & $\cdots$ & $\because$ & $\infty$ \\
\hline & $\begin{array}{l}70-01-30 \\
70-04-23\end{array}$ & 62 & 46 & 0 & 26 & 0 & $\because 0$ & $\because 0$ & 5.7 & 29 \\
\hline & $70-07-15$ & $=$ & $\ldots$ & $=$ & 16 & $\ldots$ & $\ldots$ & $\ldots$ & - & $=$ \\
\hline & $70-08-04$ & -- & -- & -- & 7 & $-\infty$ & -- & -- & -- & $-\infty$ \\
\hline & $70-09-18$ & - & -- & -- & 13 & $\infty$ & $-\infty$ & - & $-\infty$ & $\infty$ \\
\hline & $70-10-23$ & 37 & 20 & 0 & 15 & 0 & .4 & .0 & 6.4 & 16 \\
\hline & $70-12-04$ & -- & $\cdots$ & -- & 20 & 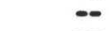 & $\cdots$ & $\cdots$ & $-\infty$ & $-\infty$ \\
\hline & $\begin{array}{l}71-01-13 \\
71-02-26\end{array}$ & 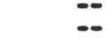 & 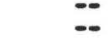 & $=$ & 11 & $=$ & $\because$ & 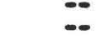 & $\because$ & $=$ \\
\hline & $71-04-28$ & - & - & -- & 25 & $-\infty$ & - & $\cdots$ & 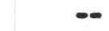 & - \\
\hline & $71-06-14$ & - & $\cdots$ & -- & 8 & - & -- & - & $-\infty$ & $\infty$ \\
\hline & $71-07-28$ & - & $=-$ & -- & 9 & -- & $=$ & 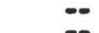 & 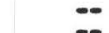 & $=$ \\
\hline & $71-09-22$ & -- & $\cdots$ & 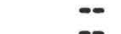 & 13 & -- & -- & $-\infty$ & $-\infty$ & $-\infty$ \\
\hline & $11-10-29$ & $\cdots$ & - & & 25 & $\cdots$ & & & $\cdots$ & $\cdots$ \\
\hline & $71-11-22$ & -- & $-\infty$ & -- & 17 & $\cdots$ & - & $\cdots$ & $-\infty$ & 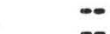 \\
\hline & $\begin{array}{l}71-12-28 \\
72-10-26\end{array}$ & $=$ & 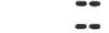 & $=$ & 31 & $=$ & $\because$ & $=$ & $=$ & 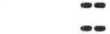 \\
\hline \multirow[t]{15}{*}{280233082342601} & $70-01-30$ & $=$ & - & 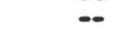 & 28 & $\ldots$ & - & 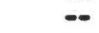 & - & - \\
\hline & $70-04-23$ & -- & - & -- & 128 & -- & -- & - & $\infty$ & $-\infty$ \\
\hline & $70-06-25$ & - & $=$ & -- & 122 & 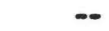 & - & $\cdots$ & - & 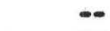 \\
\hline & $70-07-15$ & $-\infty$ & $\cdots$ & -- & 108 & $\cdots$ & $\cdots$ & $\cdots$ & $-\infty$ & $-\infty$ \\
\hline & $70-08-04$ & -- & $\cdots$ & -- & 74 & $\cdots$ & $\cdots$ & $\cdots$ & $-\infty$ & $\cdots$ \\
\hline & $70-09-18$ & -- & -- & -- & 166 & -- & -- & $\cdots$ & - & $\cdots$ \\
\hline & $70-10-26$ & -- & $\cdots$ & -- & 161 & - & $-\infty$ & $\cdots$ & $\cdots$ & $-\infty$ \\
\hline & $70-12-04$ & - & $\cdots$ & - & 35 & 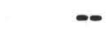 & - & $\cdots$ & $\infty$ & 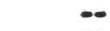 \\
\hline & $71-01-13$ & -- & -- & -- & 172 & - & $\cdots$ & -- & - & $\cdots$ \\
\hline & $71-02-26$ & $=$ & $\cdots$ & -- & 54 & -- & $=$ & $\cdots$ & $=$ & 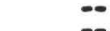 \\
\hline & $\begin{array}{l}71-03-30 \\
71=06-14\end{array}$ & 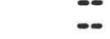 & $=$ & $\because$ & $\begin{array}{l}120 \\
181\end{array}$ & 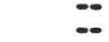 & 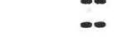 & - & $=$ & 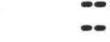 \\
\hline & $71-07-28$ & $\ldots$ & - & -- & 180 & $-\infty$ & -- & $\ldots$ & - & - \\
\hline & $71-09-22$ & - & - & - & 170 & - & $\cdots$ & $\cdots$ & $\infty$ & $\cdots$ \\
\hline & $71-10-29$ & - & $-\infty$ & $\cdots$ & 140 & $\cdots$ & -- & $-\infty$ & $-\infty$ & 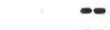 \\
\hline & $\begin{array}{l}71-11-22 \\
71-12-28\end{array}$ & $=$ & $\because$ & $=$ & 180 & 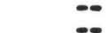 & $=$ & $\because$ & $\because$ & 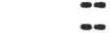 \\
\hline \multirow{3}{*}{280235082335801} & $73-04-27$ & $\ldots$ & - & - & Bu & - & - & $-\infty$ & $-\infty$ & -- \\
\hline & $73-02-01$ & 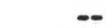 & $\ldots$ & - & 190 & - & $\infty$ & $\infty$ & $-\infty$ & - \\
\hline & $73-04-26$ & 234 & 236 & 0 & 190 & 0 & 1.6 & .2 & 14 & 15 \\
\hline \multirow[t]{6}{*}{280235082341701} & $71-01-13$ & $\cdots$ & $\cdots$ & $\cdots$ & 35 & $\cdots$ & $\cdots$ & $\infty$ & $\cdots$ & - \\
\hline & $71-10-29$ & - & $\cdots$ & -- & 140 & $-\infty$ & $\cdots$ & $-\infty$ & -- & $\cdots$ \\
\hline & $71-12-27$ & - & $\cdots$ & - & 240 & $\infty$ & $\cdots$ & $-\infty$ & $-\infty$ & $\infty$ \\
\hline & $72-01-27$ & - & 92 & 0 & 76 & 1 & $-\infty$ & $\cdots$ & $\cdots$ & 12 \\
\hline & $\begin{array}{l}72-03-16 \\
72-07-10\end{array}$ & $=$ & $=$ & 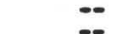 & 93 & $=$ & $\because$ & $\because$ & $\because$ & $=$ \\
\hline & $72-10-26$ & 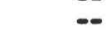 & 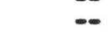 & $=$ & 78 & $\ldots$ & 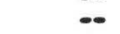 & $\cdots$ & $\infty$ & -- \\
\hline 280236082340701 & $73-02-01$ & $-\infty$ & $-\infty$ & -- & 50 & - & $-\infty$ & $\infty$ & 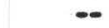 & 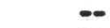 \\
\hline 280236082340901 & $73-08-01$ & $\cdots$ & $\cdots$ & -- & 180 & $\cdots$ & $-\infty$ & $\cdots$ & $-\infty$ & - \\
\hline & $73-08-10$ & - & - & - & 52 & - & -- & $-\infty$ & $-\infty$ & - \\
\hline & $\begin{array}{l}73-10-17 \\
73-12-10\end{array}$ & $\because$ & 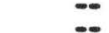 & $=$ & 54 & $\because$ & 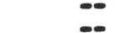 & $\because$ & $\because$ & $=$ \\
\hline \multirow{9}{*}{$\begin{array}{l}280235082341702 \\
280236082342601\end{array}$} & $72-07-19$ & - & - & -- & 100 & -- & $\ldots$ & $\ldots$ & -. & -- \\
\hline & $71-09-22$ & $\ldots$ & $\ldots$ & - & 260 & $\Longrightarrow$ & $\ldots$ & $\ldots$ & $-\infty$ & - \\
\hline & $71-10-28$ & 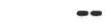 & $=$ & $\cdots$ & 160 & $\Longrightarrow$ & - & 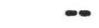 & $-\infty$ & $\infty$ \\
\hline & $72-01-27$ & - & 198 & 0 & 110 & 0 & $\cdots$ & $\cdots$ & $\infty$ & 22 \\
\hline & $72-03-16$ & - & -- & $\cdots$ & 72 & $\cdots$ & -- & -- & -0 & - \\
\hline & $72-10-26$ & 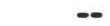 & $\ldots$ & -- & 110 & - & $\infty$ & -- & - & -- \\
\hline & $73-04-27$ & - & $\cdots$ & - & 57 & $-\infty$ & -- & $-\infty$ & 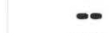 & $\cdots$ \\
\hline & $73-08-09$ & $\cdots$ & $\cdots$ & -- & 73 & $\infty$ & 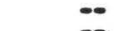 & $\infty$ & $\cdots$ & $-\infty$ \\
\hline & $\begin{array}{l}73-10-16 \\
73-12-20\end{array}$ & $=$ & $=$ & $=$ & 65 & $=$ & 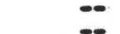 & 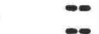 & 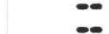 & $\because$ \\
\hline \multirow{4}{*}{$\begin{array}{l}280237082342701 \\
280237082342901 \\
280237082343801\end{array}$} & $73-04-27$ & $\ldots$ & - & - & 640 & $-\sigma^{\prime}$ & $-\infty$ & $-\infty$ & $-\infty$ & -- \\
\hline & $72-07-18$ & - & $\ldots$ & -- & 810 & - & $\ldots$ & - & -. & $\ldots$ \\
\hline & $\begin{array}{l}70-02-04 \\
70-10-22\end{array}$ & $\begin{array}{l}204 \\
210\end{array}$ & $\begin{array}{l}186 \\
216\end{array}$ & 4 & $\begin{array}{l}160 \\
180\end{array}$ & $\begin{array}{l}3 \\
3\end{array}$ & 4.4 & $\bullet 2$ & $11^{8.9}$ & $\begin{array}{l}1.5 \\
2.2\end{array}$ \\
\hline & $72-07-18$ & & 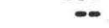 & $\infty$ & 130 & 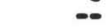 & 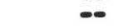 & $\ldots$ & 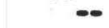 & $-\infty$ \\
\hline
\end{tabular}


Table 6B,--Water-quality data for ground-water sites at the Rocky CReek Landfill---Continued

\begin{tabular}{|c|c|c|c|c|c|c|c|c|c|c|}
\hline STATION NUMBER & $\begin{array}{c}\text { DAIE } \\
\text { OF } \\
\text { SAMPLE }\end{array}$ & $\begin{array}{l}\text { SOL IDS, } \\
\text { REEIDUE } \\
\text { AT } 180 \\
\text { OEG. } \\
\text { DIS- } \\
\text { SOLVED } \\
\text { (MU/L.) }\end{array}$ & $\begin{array}{l}\text { BICAR- } \\
\text { BONATE } \\
\text { (MGAL } \\
\text { AS } \\
\text { HCO3) }\end{array}$ & $\begin{array}{l}\text { CAR- } \\
\text { BONATE } \\
\text { (MG/L } \\
\text { AS COB) }\end{array}$ & $\begin{array}{l}\text { HARD- } \\
\text { NESS } \\
\text { (MG/L } \\
\text { AS } \\
\text { CAC031 }\end{array}$ & $\begin{array}{l}\text { HARU- } \\
\text { NESS, } \\
\text { NONCAR- } \\
\text { BONATE } \\
\text { (MG/L } \\
\text { CACO3) }\end{array}$ & $\begin{array}{l}\text { SULFATE } \\
\text { OIS- } \\
\text { SOLED } \\
\text { MMG/L } \\
\text { AS SO4) }\end{array}$ & $\begin{array}{l}\text { FLUO- } \\
\text { RIDE, } \\
\text { OIS- } \\
\text { SOLVED } \\
\text { (MG/L } \\
\text { AS F) }\end{array}$ & $\begin{array}{l}\text { SILICA, } \\
\text { OIIS- } \\
\text { SOLVED } \\
\text { IMG/L } \\
\text { AS } \\
\text { SIOZI }\end{array}$ & $\begin{array}{c}\text { CARBON } \\
\text { DIOXIOE } \\
\text { DIS- } \\
\text { SOLVED } \\
\text { (MG/L } \\
\text { AS COL) }\end{array}$ \\
\hline \multirow[t]{2}{*}{280237082343801} & $72-10-25$ & -- & -- & -- & 120 & -- & $-\cdot$ & -- & -- & $=$ \\
\hline & 7 & 200 & 200 & $\overline{0}$ & $\begin{array}{l}140 \\
170\end{array}$ & $\overline{4}$ & 1.6 & .2 & $8 . \overline{5}$ & 8.0 \\
\hline \multirow[t]{2}{*}{280237082343802} & $\begin{array}{l}70-01-20 \\
70-02-04\end{array}$ & 208 & $20 \overline{8}$ & $\overline{0}$ & $\begin{array}{r}82 \\
160\end{array}$ & $\overline{0}$ & $10^{--}$ & .2 & $2 . \overline{0}$ & $17^{-0}$ \\
\hline & $\begin{array}{l}70-10-27 \\
73-02-01\end{array}$ & $\because$ & $\because$ & $=$ & $\begin{array}{l}81 \\
46\end{array}$ & $\because$ & $\because$ & $\because$ & $\because$ & $\because$ \\
\hline \multirow[t]{2}{*}{280237082343803} & $\begin{array}{l}73-04-24 \\
70-01-20\end{array}$ & 62 & 46 & $\therefore$ & $\begin{array}{l}42 \\
18\end{array}$ & $\therefore$ & 8.0 & $=$ & 2.7 & ${ }^{23} \ldots$ \\
\hline & $70-02-04$ & 51 & 36 & 0 & & 2 & 6.0 & .1 & 4.0 & \\
\hline \multirow[t]{3}{*}{280238082340902} & $\begin{array}{l}72-07-18 \\
73-08-01\end{array}$ & $=$ & $=$ & $=$ & $\begin{array}{l}180 \\
220\end{array}$ & $=$ & $\because$ & $=$ & $\overline{-}$ & $\because$ \\
\hline & $\begin{array}{l}73-08-10 \\
73-10-17\end{array}$ & $=$ & $=$ & $=$ & $\begin{array}{l}130 \\
140\end{array}$ & $\because$ & $=$ & $=$ & $=$ & $=$ \\
\hline & $73-12-19$ & -- & -- & -- & 130 & -- & -- & -- & -- & -- \\
\hline \multirow[t]{2}{*}{280238082340901} & 73-08-01 & $=$ & $=$ & -- & 190 & $=$ & $\because-$ & $=$ & $=$ & $\because-$ \\
\hline & $73-10-17$ & -- & -- & -- & 190 & -- & -- & - & -- & -- \\
\hline \multirow[t]{7}{*}{280238082343202} & $70-02-04$ & 163 & 166 & 0 & $\begin{array}{l}127 \\
127-10\end{array}$ & 0 & 8.0 & .1 & 2.0 & $27^{--}$ \\
\hline & $70-10-22$ & -- & -- & -- & 75 & -- & $=$ & $\ddot{-}+2+x$ & 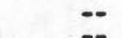 & $=$ \\
\hline & $71-02-24$ & $=$ & $=$ & $=$ & 67 & $\because$ & $=$ & $=$ & $\ldots$ & $=$ \\
\hline & $\begin{array}{l}71-09-21 \\
71-11-22\end{array}$ & $\because$ & $=$ & $=$ & 114 & $=$ & $=$ & $=$ & $=$ & $=$ \\
\hline & $71-12-27$ & -- & -- & $=$ & 96 & $=$ & $=$ & $=$ & $=$ & $=$ \\
\hline & $\begin{array}{l}72-01-26 \\
72-03-16\end{array}$ & $=$ & $=$ & $=$ & $\begin{array}{r}62 \\
290\end{array}$ & $\because$ & $=$ & $=$ & $=$ & $\cdots$ \\
\hline & $\begin{array}{l}72-06-22 \\
72-07-18\end{array}$ & $=$ & $=$ & $=$ & $\begin{array}{r}290 \\
36\end{array}$ & $=$ & $=$ & $=$ & $=$ & $\because$ \\
\hline \multirow{16}{*}{280238082343201} & $72-07-21$ & -- & -- & -- & 62 & -- & -- & -- & - & -- \\
\hline & $\begin{array}{l}70-01-07 \\
70-02-02\end{array}$ & 37 & $\ddot{10}$ & $\ddot{0}$ & $\begin{array}{r}14 \\
7\end{array}$ & $\overline{0}$ & 3.2 & $\ddot{.2}$ & $1 . \overline{9}$ & $13^{--}$ \\
\hline & $\begin{array}{l}70-02-04 \\
70-09-17\end{array}$ & 226 & 230 & 0 & 186 & $\therefore$ & $\therefore 0$ & $\therefore 2$ & ${ }^{14} \ldots$ & $4: 0$ \\
\hline & $70-10-22$ & 230 & 220 & 4 & 78 & 2 & 1.1 & .2 & 14 & 9.2 \\
\hline & $\begin{array}{l}70-12-02 \\
71-01-14\end{array}$ & $=$ & $=$ & $=$ & 170 & $\because-$ & $=$ & $\because$ & $\because$ & $=$ \\
\hline & $\begin{array}{l}71-02-24 \\
71-03-31\end{array}$ & $=$ & $=$ & $=$ & $\begin{array}{r}76 \\
198\end{array}$ & $=-$ & $=$ & $=$ & $\because$ & $\because-$ \\
\hline & $71-04-28$ & -- & -- & -- & 189 & -- & -- & -- & - & - \\
\hline & 71 & -- & -- & -- & 88 & -- & - & - & $=$ & $\cdots$ \\
\hline & $\begin{array}{l}11-01-28 \\
71-09-21\end{array}$ & $=$ & $=$ & $=$ & $\begin{array}{l}188 \\
195\end{array}$ & $=$ & $=$ & $=$ & $=$ & $\ldots$ \\
\hline & $71-09-22$ & -- & -- & -- & 42 & -- & -- & -- & $\cdots$ & -- \\
\hline & $\begin{array}{l}71-10-28 \\
71-11=23\end{array}$ & $=$ & $\ddot{z}+1+x$ & $=$ & 40 & $=$ & $=$ & $=$ & $=$ & $=-$ \\
\hline & $71-12-27$ & - & - & - & 130 & - & - & - & $\cdots$ & 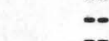 \\
\hline & $72-06-20$ & $=$ & $=$ & $=$ & $\begin{array}{l}530 \\
180\end{array}$ & $=$ & $=$ & $=$ & $=$ & $=$ \\
\hline & $73-02-01$ & $=$ & $=$ & -- & 190 & $=$ & $\because$ & $=$ & $=$ & $=$ \\
\hline & & $=$ & - & $=$ & 180 & $=$ & - & - & - & $\cdots$ \\
\hline & $70=01-07$ & $=$ & $=$ & $=$ & ${ }_{14}^{220}$ & $=$ & $=$ & $=$ & -- & $\cdots$ \\
\hline \multirow{2}{*}{280238082342603} & $70-09-17$ & -- & -- & -- & 21 & $=$ & $=$ & 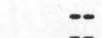 & $=$ & $=$ \\
\hline & $71-10-28$ & $=$ & - & $=$ & $\begin{array}{l}4 c \\
40\end{array}$ & $=$ & $=$ & $=$ & $=$ & $=$ \\
\hline \multirow[t]{13}{*}{280238082342602} & $70=01=07$ & $\ldots$ & -- & $=$ & $\begin{array}{r}400 \\
50\end{array}$ & -- & $=$ & $=$ & $=$ & $\cdots$ \\
\hline & $70-02-02$ & 65 & 58 & 0 & 20 & 0 & .0 & .1 & 5.1 & 29 \\
\hline & $\begin{array}{l}70-10-22 \\
71-01-13\end{array}$ & 38 & 30 & $\therefore$ & $\begin{array}{r}15 \\
9\end{array}$ & 0 & 6.3 & $\cdot 2$ & 5.9 & 7.6 \\
\hline & $\begin{array}{l}71-10-28 \\
71-11-22\end{array}$ & $=$ & $\because$ & $=$ & $\begin{array}{l}100 \\
120\end{array}$ & $=$ & $=$ & $=$ & $=$ & $=$ \\
\hline & $71-12-27$ & - & $=$ & - & 10 & -- & -- & $\cdots$ & -- & $=$ \\
\hline & $\begin{array}{l}72-01-27 \\
72-03-16\end{array}$ & $=$ & 156 & $\therefore$ & $\begin{array}{l}14 \\
14\end{array}$ & $\therefore$ & $=$ & $\because$ & $=$ & 7 \\
\hline & $\begin{array}{l}72-06-21 \\
72-07-18\end{array}$ & $\cdots$ & $=$ & $=$ & $\begin{array}{l}20 \\
90\end{array}$ & $=$ & $=$ & $=$ & $\because$ & $=$ \\
\hline & $72-08-08$ & -- & -- & -- & 17 & -- & -- & -- & -- & -- \\
\hline & $\begin{array}{l}72-09-06 \\
72-10-26\end{array}$ & -- & -- & $\cdots$ & $\begin{array}{l}12 \\
13\end{array}$ & -- & $=$ & $\cdots$ & -- & $\cdots$ \\
\hline & $\begin{array}{l}73-02-11 \\
73-04-27\end{array}$ & -- & $=$ & $=$ & $\underset{8}{11}$ & $=$ & $=$ & $=$ & $\because$ & $\because$ \\
\hline & 8- & -- & -- & - & 10 & -- & -- & 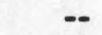 & -- & $\cdots$ \\
\hline & & -- & -- & -- & 19 & $=$ & $z$ & $=$ & $=$ & -- \\
\hline & $70-02-02$ & 168 & 166 & 0 & 138 & 2 & 8.0 & .2 & 2.6 & 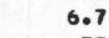 \\
\hline
\end{tabular}


TABle 6B,--WATER-Quality data foR GROUND-WATER Sites at the Rocky CREek LANDFill--Continued

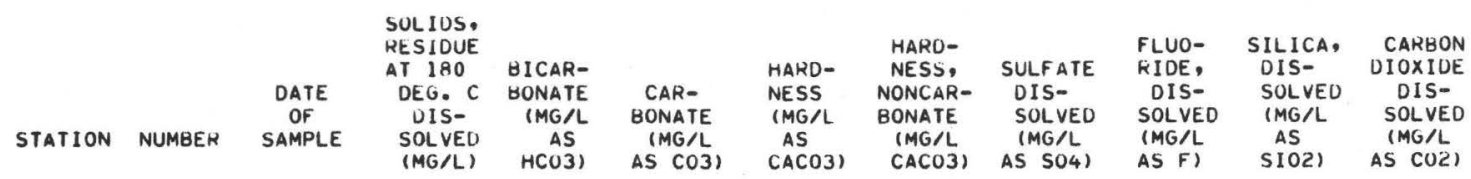

280238082342601

$\begin{array}{lllll}70-09-17 & -- & -- & -- & 1 \\ 70-10-22 & 208 & 212 & - & 1 \\ 70-12-03 & -- & -- & -- & 2014 \\ 71-01-13 & -- & -- & -- & \\ 71-02-26 & -- & -- & -- & 2084 \\ 71-03-30 & -- & -- & -- & 233 \\ 71-04-28 & -- & -- & -- & 1 \\ 71-06-14 & -- & -- & -- & 1 \\ 71-07-27 & -- & -- & - & \end{array}$

189

189
174
141
208

--
--
--
-
-
-
--

$\square-9$
$\because-$
-
$\because-$
$\because-$
-

$71-10-28$

$71-11-22$

$72-06-21$

$72-07-18$
$72-10-20$

$--\quad--\quad--\quad 250$

208
233
194
211
150
220
150
190
290
220

208
233
194
211
150
220
150
190
290
220

73-02-01

$73-04-27$
$73-08-13$
$73-10-16$
$73-12-20$

$73-04-27$
$73-08-13$
$73-10-16$
$73-12-20$

$73-04-27$
$73-08-13$
$73-10-16$
$73-12-20$

$\begin{array}{ll}-- & 200 \\ -- & 200 \\ -- & 180 \\ - & 200\end{array}$

280238082335701

73-02-01

$73-04-25$

$73-08-13$

73-10-16

$73-12-21 \quad=-$

280238082342205

70-08- 04

$70-09-18$

$70-10-26$

$70-12-03$
$71-01-13$

$71-02-24$

$71-03-31$

$71-04-28$

$71-04-28$
$71-06-14$

$71-07-27$

$73-04-26$
$73-12-20$

$73-12-20$
$70-04-08$

280238082342204

$70-09-18$

$70-10-26 \quad--$

180

ב-

$\overline{--}$

$70-12-03$

$71-01-13$

$71-03-31$

$71-04-28$

$71-06-14$

190

$\begin{array}{cl}-- & 190 \\ 0 & 180\end{array}$

$\ddot{--}$

180
170
210

$\begin{array}{rr}- & 83 \\ - & 139 \\ -- & 157 \\ - & 133\end{array}$

83
139
157
133
179

ב-

$\begin{array}{ll}-- & -- \\ - & -- \\ -- & -\end{array}$

$\begin{array}{ll}-- & -5 \\ -- & 1.6 \\ -- & =\end{array}$

$\begin{array}{lr}-- & 47 \\ -- & 170 \\ -- & 190 \\ - & 142\end{array}$

$71-07-27$

$71-11-22$

$72-07-19$
$73-04-27$

280238082342203

$73-04-27$

70-09-18

$70-10-26$

$70-12-03$

$71-01-13$

$71-02-24$

$71-03-31$

$71-04-28$

$71-06-14$

$71-07-27$

$71-09-22$

71-10-29

$72-01-27$

$72-07-20$
$73-04-26$

$280240082340601 \quad \begin{aligned} & 73-04-26 \\ & 71-12-28\end{aligned}$

$72-01-26$

72-03-16

$72-07-17$

$72-07-19$

72-10-25

$72-10-26$

73-02-01

73-04-23

73-04-25

73-08-09

$\because \because \because \because$

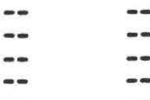

$\because$

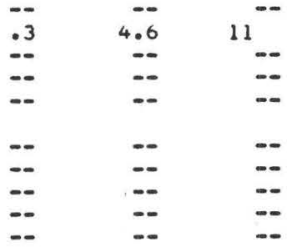

$73-08-13$
$73-10-15$

$73-10-16$
$73-12-19$

73-12-20

150
180
90
39
40

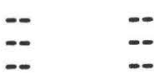

$=$

--

$\overline{-}$

$\begin{array}{ll}-- & - \\ -- & -\end{array}$

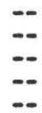

$\because=$

$\begin{array}{lll}- & - & - \\ - & -\end{array}$

-
$\because$
-

$\because$

$14^{--} \quad 7.2$

$\because=$

$\because \quad \because=\quad \because$

$\because$

$\because$

$\because$

$\because=$

$\because \quad \because=\quad \because=$

$\because=\quad \because \because$

53

$\begin{array}{r}27 \\ -\quad 38 \\ \hline\end{array}$

32

$-$

$\because \quad=$

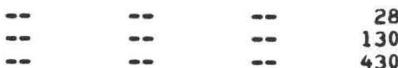

130
430
200

$\begin{array}{lll}-- & - \\ z & -\end{array}$

-

$\begin{array}{rrrr}-- & - & - & 52 \\ -- & -- & - & 192\end{array}$

$\begin{array}{rrrr}-z & = & = & 123 \\ -- & = & = & 191 \\ & -- & -- & 27\end{array}$

$\begin{array}{ll}\because & = \\ z & =\end{array}$

$\begin{array}{lll}- & = & = \\ - & = & = \\ - & = & =\end{array}$

208
178
197
193

-

290

$\begin{array}{ll}-- & 290 \\ - & -- \\ - & -\end{array}$

-- 200

200

200

200
190

190

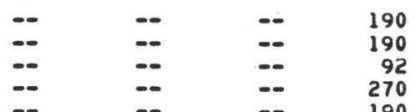

$\begin{array}{lll}- & - \\ \therefore & -\end{array}$

$\because$

$\therefore=$

$\ddot{\square}$

$\because=$

$\because \quad=$

$\begin{array}{llll}- & - & -- & 39 \\ - & -- & = & 11\end{array}$

$\begin{array}{lll}- & = & = \\ - & = & =\end{array}$

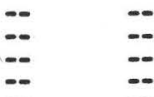

190

$-$

$\because \because$

$\begin{array}{lll}- & = & = \\ z & = & = \\ - & = & =\end{array}$

43
190
8
180
13

$\ddot{-\infty}$ 
Table 6b, --Hater-quality data for ground-water sites at the Rocky Creek landfill--Continued

\begin{tabular}{|c|c|c|c|c|c|c|c|c|c|c|}
\hline STATION NUMBER & $\begin{array}{c}\text { UATE } \\
\text { OF } \\
\text { SAMPLE }\end{array}$ & $\begin{array}{l}\text { SOL 1DS, } \\
\text { HESIOUE } \\
\text { AT } 180 \\
\text { DEG. C } \\
\text { DIS- } \\
\text { SUL VED } \\
\text { (MG/L) }\end{array}$ & $\begin{array}{l}\text { OICAR- } \\
\text { BONATE } \\
\text { (MG/L } \\
\text { AS } \\
\text { HCO3) }\end{array}$ & $\begin{array}{l}\text { CAR- } \\
\text { BONATE } \\
\text { (MGL } \\
\text { AS CO3) }\end{array}$ & $\begin{array}{l}\text { HARD- } \\
\text { NESS } \\
\text { (MG/L } \\
\text { AS } \\
\text { CACO3) }\end{array}$ & $\begin{array}{l}\text { HARU- } \\
\text { NESS, } \\
\text { NONCAR- } \\
\text { BONATE } \\
\text { (MGLL } \\
\text { CACO3) }\end{array}$ & $\begin{array}{l}\text { SULFATE } \\
\text { DIS- } \\
\text { SOLVED } \\
\text { (MG/L } \\
\text { AS SO4) }\end{array}$ & $\begin{array}{l}\text { FLUO- } \\
\text { RIDE, } \\
\text { UIS- } \\
\text { SOLVED } \\
\text { (MG/L } \\
\text { AS F) }\end{array}$ & $\begin{array}{l}\text { SILICA. } \\
\text { DIS- } \\
\text { SOLVED } \\
\text { (MGL } \\
\text { AS } \\
\text { SIO2) }\end{array}$ & $\begin{array}{l}\text { CARBON } \\
\text { OIOXIOE } \\
\text { OIS- } \\
\text { SOLVEU } \\
\text { (MG/L } \\
\text { AS CO2) }\end{array}$ \\
\hline 280240082340602 & $\begin{array}{l}73-01-02 \\
73-04-25 \\
73-08-13 \\
73-10-16 \\
73-12-19\end{array}$ & 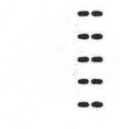 & $\begin{array}{l}= \\
= \\
=\end{array}$ & 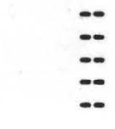 & $\begin{array}{l}190 \\
210 \\
200 \\
220 \\
200\end{array}$ & $\begin{array}{l}= \\
= \\
=\end{array}$ & $\begin{array}{l}= \\
=- \\
=\end{array}$ & 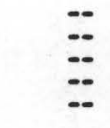 & $\begin{array}{l}= \\
= \\
=\end{array}$ & $=$ \\
\hline $\begin{array}{l}280240082341202 \\
280240082341201\end{array}$ & $\begin{array}{l}73-08-10 \\
73-12-19 \\
73-08-01 \\
73-08-10 \\
73-10-16\end{array}$ & $\begin{array}{l}\because \\
= \\
=\end{array}$ & $\begin{array}{l}a \\
- \\
- \\
-\end{array}$ & $\begin{array}{l}= \\
=- \\
=\end{array}$ & $\begin{array}{r}200 \\
180 \\
190 \\
140 \\
50\end{array}$ & $\begin{array}{l}= \\
= \\
= \\
=\end{array}$ & $\begin{array}{l}\because \\
= \\
=\end{array}$ & $=$ & $\begin{array}{l}= \\
= \\
=\end{array}$ & 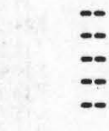 \\
\hline 280240082335901 & $\begin{array}{l}73-12-19 \\
72-07-17 \\
72-10-26 \\
73-02-01 \\
73-04-25\end{array}$ & 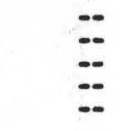 & $\begin{array}{l}\because \\
\because \\
-\end{array}$ & $\begin{array}{l}= \\
\because \\
=\end{array}$ & $\begin{array}{r}95 \\
16 \\
18 \\
9 \\
34\end{array}$ & $\begin{array}{l}= \\
= \\
=\end{array}$ & $\begin{array}{l}= \\
= \\
=\end{array}$ & $=$ & $\begin{array}{l}= \\
=- \\
=\end{array}$ & 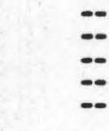 \\
\hline $\begin{array}{l}280240082335902 \\
280239082343503\end{array}$ & $\begin{array}{l}73-02-01 \\
73-04-25 \\
70-02-05 \\
72-06-22 \\
73-02-01\end{array}$ & 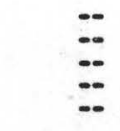 & $\begin{array}{l}= \\
\therefore \\
-\end{array}$ & $\begin{array}{l}= \\
= \\
=\end{array}$ & $\begin{array}{r}310 \\
240 \\
24 \\
62 \\
190\end{array}$ & $\begin{array}{l}\ddot{-} \\
-- \\
--\end{array}$ & 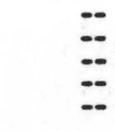 & 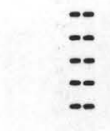 & $\begin{array}{l}= \\
=- \\
=\end{array}$ & $\begin{array}{l}\because \\
\therefore \\
\end{array}$ \\
\hline 280239082343502 & $\begin{array}{l}72-06-20 \\
72-07-18 \\
72-08-08 \\
72-09-06 \\
72-10-25\end{array}$ & 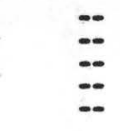 & $\begin{array}{l}= \\
= \\
= \\
=\end{array}$ & $\begin{array}{l}= \\
= \\
=\end{array}$ & $\begin{array}{l}100 \\
170 \\
510 \\
380 \\
130\end{array}$ & $\begin{array}{l}= \\
= \\
=\end{array}$ & $\begin{array}{l}\because \\
\because \\
=\end{array}$ & 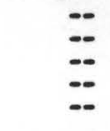 & $\begin{array}{l}= \\
= \\
=\end{array}$ & 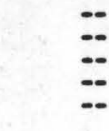 \\
\hline 280239082343501 & $\begin{array}{l}73-04-24 \\
70-02-05 \\
72-06-20 \\
72-07-18 \\
72-10-25\end{array}$ & 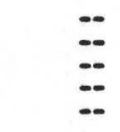 & $\begin{array}{l}= \\
= \\
=\end{array}$ & $\begin{array}{l}= \\
= \\
=\end{array}$ & $\begin{array}{r}130 \\
50 \\
190 \\
400 \\
370\end{array}$ & $\begin{array}{c}-- \\
0 \\
--\end{array}$ & $\begin{array}{l}= \\
= \\
=\end{array}$ & 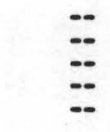 & $\begin{array}{l}= \\
= \\
=\end{array}$ & $=$ \\
\hline 280240082342601 & $\begin{array}{l}73-04-24 \\
73-12-19 \\
70-01-08 \\
70-02-03 \\
70-09-17\end{array}$ & 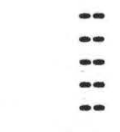 & $\begin{array}{l}= \\
= \\
=\end{array}$ & $\begin{array}{l}= \\
= \\
=\end{array}$ & $\begin{array}{r}180 \\
220 \\
95 \\
175 \\
112\end{array}$ & $\begin{array}{l}= \\
\ddot{z} \\
=\end{array}$ & $\begin{array}{l}= \\
= \\
=\end{array}$ & $\begin{array}{l}= \\
\therefore \\
=\end{array}$ & $\begin{array}{l}= \\
= \\
=\end{array}$ & 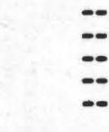 \\
\hline & $\begin{array}{l}70-10-26 \\
71-01-13 \\
71-02-26 \\
71-03-30 \\
71-04-28\end{array}$ & 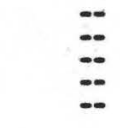 & $\begin{array}{l}= \\
= \\
=\end{array}$ & 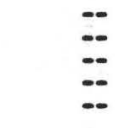 & $\begin{array}{r}136 \\
93 \\
59 \\
175 \\
198\end{array}$ & $\begin{array}{l}= \\
=- \\
=\end{array}$ & $\begin{array}{l}= \\
\because \\
=\end{array}$ & $\begin{array}{l}- \\
= \\
=\end{array}$ & $\begin{array}{l}= \\
= \\
=\end{array}$ & $=$ \\
\hline & $\begin{array}{l}71-06-14 \\
71-07-27 \\
71-12-27 \\
72-01-27 \\
72-03-15\end{array}$ & $=$ & 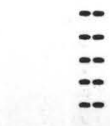 & $\begin{array}{l}= \\
= \\
=\end{array}$ & $\begin{array}{l}172 \\
196 \\
150 \\
190 \\
670\end{array}$ & $\begin{array}{l}= \\
= \\
= \\
=\end{array}$ & $\begin{array}{l}= \\
= \\
=\end{array}$ & $\begin{array}{l}- \\
= \\
=\end{array}$ & $\begin{array}{l}\because \\
= \\
\end{array}$ & 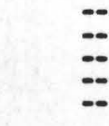 \\
\hline & $\begin{array}{l}72-06-21 \\
72-07-18 \\
72-08-08 \\
72-09-06 \\
73-04-27\end{array}$ & $\therefore$ & $\begin{array}{l}= \\
\because \\
\because\end{array}$ & $\begin{array}{l}\because \\
\because \\
=\end{array}$ & $\begin{array}{r}220 \\
290 \\
570 \\
320 \\
200\end{array}$ & $\begin{array}{l}\because \\
\because \\
\because\end{array}$ & $\begin{array}{l}= \\
= \\
=\end{array}$ & $=$ & $\begin{array}{l}= \\
= \\
=\end{array}$ & $=$ \\
\hline $\begin{array}{l}280240082342602 \\
280242082343503\end{array}$ & $\begin{array}{l}72-10-26 \\
73-08-13 \\
73-12-20 \\
70-02-06 \\
71-09-21\end{array}$ & $\begin{array}{c}\therefore \\
\therefore\end{array}$ & 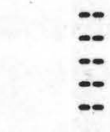 & $\begin{array}{l}= \\
= \\
=\end{array}$ & $\begin{array}{l}58 \\
39 \\
47 \\
35 \\
38\end{array}$ & $\begin{array}{l}= \\
= \\
=\end{array}$ & $\begin{array}{l}= \\
= \\
=\end{array}$ & $=$ & 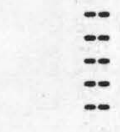 & $\ddot{=}$ \\
\hline 280242082343502 & $\begin{array}{l}72-01-26 \\
72-07-20 \\
70-01-22 \\
70-02-06 \\
71-03-31\end{array}$ & 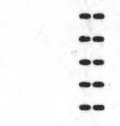 & $\begin{array}{l}56 \\
-\therefore \\
=- \\
-0\end{array}$ & $\begin{array}{l}0 \\
\because \\
\therefore\end{array}$ & $\begin{array}{l}62 \\
62 \\
48 \\
81 \\
38\end{array}$ & $\begin{array}{r}16 \\
0 \\
0 \\
0\end{array}$ & $\begin{array}{l}= \\
= \\
=\end{array}$ & $=$ & $\begin{array}{l}= \\
= \\
=\end{array}$ & 11. \\
\hline & $\begin{array}{l}71-04-28 \\
71-06-15 \\
71-09-21 \\
71-10-28 \\
72-03-16\end{array}$ & $\begin{array}{l}\because \\
\therefore \\
0\end{array}$ & $\begin{array}{l}\because \\
\because \\
\because\end{array}$ & $=$ & $\begin{array}{r}38 \\
31 \\
54 \\
52 \\
110\end{array}$ & $\begin{array}{l}= \\
= \\
=\end{array}$ & $\begin{array}{l}= \\
= \\
=\end{array}$ & $\begin{array}{l}\because \\
\therefore \\
0\end{array}$ & $\begin{array}{l}= \\
= \\
=\end{array}$ & 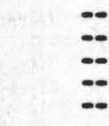 \\
\hline & $\begin{array}{l}72-06-20 \\
72-08-08 \\
72-09-06 \\
72-10-25 \\
73-02-01\end{array}$ & 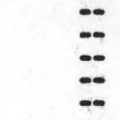 & 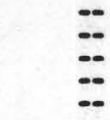 & $=$ & $\begin{array}{r}24 \\
110 \\
120 \\
73 \\
49\end{array}$ & $\begin{array}{l}= \\
= \\
=\end{array}$ & 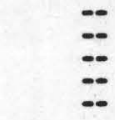 & $\begin{array}{l}- \\
- \\
-\end{array}$ & $\begin{array}{l}= \\
= \\
=\end{array}$ & $\ddot{-\infty}$ \\
\hline & $\begin{array}{l}73-04-24 \\
73-08-13 \\
73-10-02 \\
73-10-15 \\
73-12-20\end{array}$ & $\begin{array}{l}\because \\
0 \\
0\end{array}$ & $\ddot{\square}$ & 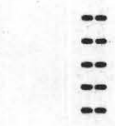 & $\begin{array}{r}120 \\
55 \\
0- \\
79 \\
110\end{array}$ & $\begin{array}{l}= \\
= \\
=\end{array}$ & 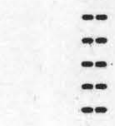 & 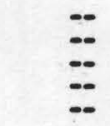 & $\begin{array}{l}-8 \\
8.5 \\
0 \\
-.\end{array}$ & 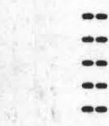 \\
\hline 280242082343501 & $\begin{array}{l}70-02-06 \\
71-03-31 \\
71-04-28 \\
71-06-15 \\
71-07-28\end{array}$ & $\because$ & $\begin{array}{l}\because \\
\because \\
\ddot{0}\end{array}$ & $\begin{array}{l}= \\
= \\
=\end{array}$ & $\begin{array}{l}188 \\
188 \\
201 \\
190 \\
186\end{array}$ & $\begin{array}{l}0 \\
\because \\
\because\end{array}$ & $\begin{array}{l}\because \\
\because \\
\end{array}$ & $=$ & $\begin{array}{l}= \\
= \\
=\end{array}$ & $\begin{array}{l}\because \\
\because \\
\because\end{array}$ \\
\hline
\end{tabular}


Table 6B,--Water-quality data for ground-Water sites at the Rocky Creek Landfill--Continued

\begin{tabular}{|c|c|c|c|c|c|c|c|c|c|c|c|}
\hline STATION & NUMBER & $\begin{array}{c}\text { DATE } \\
\text { OF } \\
\text { SAMPLE }\end{array}$ & $\begin{array}{l}\text { SOLIJS, } \\
\text { RESIDUE } \\
\text { AT } 1 \text {, } \\
\text { DEG. C } \\
\text { OIS- } \\
\text { SOLVED } \\
\text { (MG/L) }\end{array}$ & $\begin{array}{c}\text { BICAR- } \\
\text { BONATE } \\
\text { (MG/L } \\
\text { AS } \\
\text { HCO3) }\end{array}$ & $\begin{array}{l}\text { CAR- } \\
\text { BONATE } \\
\text { (MGL } \\
\text { AS CO3) }\end{array}$ & $\begin{array}{l}\text { HARD- } \\
\text { NESS } \\
\text { (MG/L } \\
\text { AS } \\
\text { CACO3) }\end{array}$ & $\begin{array}{l}\text { HARU- } \\
\text { NESS, } \\
\text { NONCAR- } \\
\text { BONATE } \\
\text { (MG/L } \\
\text { CACO3) }\end{array}$ & $\begin{array}{l}\text { SULFATE } \\
\text { DIS- } \\
\text { SOLVEO } \\
\text { (MG/L } \\
\text { AS SO4) }\end{array}$ & $\begin{array}{l}\text { FLUO- } \\
\text { RIDE, } \\
\text { OIS- } \\
\text { SOLVED } \\
\text { (MG/L } \\
\text { AS F) }\end{array}$ & $\begin{array}{l}\text { SILICA, } \\
\text { UIS- } \\
\text { SOLVED } \\
\text { (MG/L } \\
\text { AS } \\
\text { SI02) }\end{array}$ & $\begin{array}{c}\text { CARBON } \\
\text { OIOXIDE } \\
\text { DIS- } \\
\text { SOLVED } \\
\text { (MG/L } \\
\text { AS COZ) }\end{array}$ \\
\hline & & & & & & & & & & & \\
\hline
\end{tabular}

280242082343501

$\begin{array}{llll}71-09-21 & -- & -- & -- \\ 71-10-28 & -- & -- & - \\ 72-03-16 & - & -- & - \\ 72-06-20 & - & -- & - \\ 72-08-08 & - & -- & -\end{array}$

72-09-06 $72-10-25$ $73-04-24$ $73-08-09$ 73-10-15 $73-10-24$
$73-12-20$ 280245082342701 $71-10-28$ $72-01-27$ 72-03-15

280242082342601 280242082342601070 10-02-02 $70-09-17$
$70-10-26$ $70-10-26$
$70-12-03$ $71-01-13$

$71-02-26$ $71-03-30$ $71-04-28$ $71-06-14$ $71-07-27$

$71-10-28$ $71-12-27$ $72-01-27$ $72-06-22$ $72-07-18$ 72-07-20 $72-10-26$ $73-04-23$ $73-08-10$ 73-10-16

73-12-19

280242082342602 $70-01-12$
$70-02-02$ $70-02-02$
$70-09-17$ $70-10-26$

$71-02-20$ $71-07-27$ $71-09-22$ $71-10-28$ $71-10-28$
$71-12-27$

$72-03-15$ $72-04-23$
$72-06-22$

$280246082343202 \quad \begin{array}{r}70-02-06 \\ 70-09-17\end{array}$ -

$70-10-22$ $70-12-02$ $71-01-14$ $71-02-24$
$71-03-31$

$72-07-18$ $73-04-24$ $73-08-13$ 73-08-13 73-10-15

280243082342501

70-09-17 $71-11-22$ $71-12-27$ $72-01-26$
$72-07-19$

$72-10-26$ 73-08-09 $73-10-16$
$73-12-19$

$280243082342602 \quad 70-01-13$

70-02-03 70-09-17 $70-09-17$
$71-09-22$ $71-09-22$
$71-10-28$ $72-06-21$

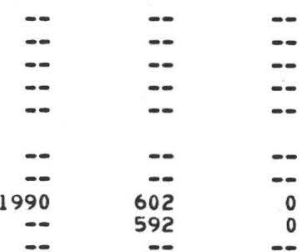

$\begin{array}{lll}212 & -- & = \\ 200 & = & = \\ 620 & =- & = \\ 200 & = & -\end{array}$

$\begin{array}{lll}\because & \because & \because \\ \because & \because & \because \\ \because & \because & =\end{array}$

55
108

$\begin{array}{lll}-- & - & - \\ - & - & -\end{array}$

$\begin{array}{lll}- & = & = \\ z & = & =\end{array}$

$\begin{array}{lll}-- & -- & --\end{array}$

200
200

$174 \quad 174$

$\begin{array}{cc}174 & 174 \\ -- & - \\ -- & -\end{array}$

0
--
--

73-04-27

$70-10-22$

$72-01-26$

$72-01-26$
$73-02-01$
200

630

190
190

180

210
694
740
1900

112

126

55

120

158

191

120

130

200
180

210

180

180
190

170

14

40
36
37

(a)

26
21

84
19

29

190
78
44

44
141

140

196

159
63

194

230

180

180
180

180

34
48
71
74

59

93
51

51
18
98

98
15

32

34

120
32

$=$

199

-- --

$\overline{--}$

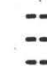

$\because$

$-$

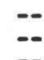

$=$

$\overline{-}$

$\because$

$--\quad-$

$\ddot{--}$

$\overline{-}$

$\because=$

$=$

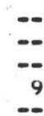

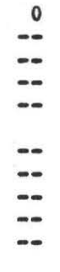

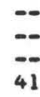

$\overline{41}$

$\begin{array}{ll}z & = \\ z & = \\ -- & =\end{array}$

$\ddot{-}$

180
150

190

63

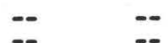

$\begin{array}{lll}- & = & = \\ z & \because & = \\ z & = & = \\ z & =\end{array}$

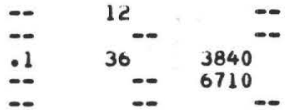

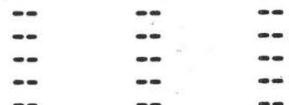

$\begin{array}{lll}z & = & =\end{array}$

$\begin{array}{lll}-z & = & = \\ z & =\end{array}$

$\begin{array}{lll}- & = & = \\ z & = & =\end{array}$

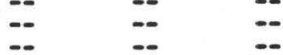

$\ddot{z} \quad=\quad=$

$\begin{array}{lll}- & = & = \\ z & =\end{array}$

$\begin{array}{lll}-- & - & - \\ - & -\end{array}$

$\begin{array}{lll}- & - & - \\ - & - & -\end{array}$

$\begin{array}{lll}- & - & - \\ - & -\end{array}$

$\begin{array}{lll}-Z & = & = \\ 6.0 & = & = \\ \because- & = & =\end{array}$

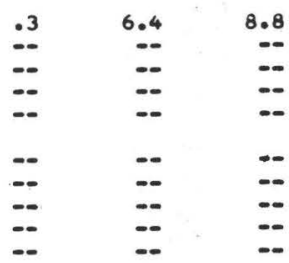

$\begin{array}{lll}-- & - & -\end{array}$

$\begin{array}{lll}\because & \because & = \\ = & = & 10\end{array}$

$\because \quad=\quad \because$

$\begin{array}{lll}z & = & = \\ z & =\end{array}$

$\begin{array}{lll}- & - & -\end{array}$

$\because \quad=\quad=$

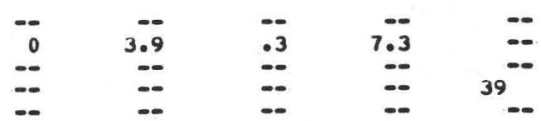

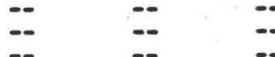

$\begin{array}{lll}- & - & -\end{array}$

$\because \quad \because=\quad \because$ 
Table 6B,--Water-quality data for ground-water sites at the Rocky Creek landfill--Continued

\begin{tabular}{|c|c|c|c|c|c|c|c|c|c|c|}
\hline STATION NUMBER & $\begin{array}{c}\text { DAIE } \\
\text { OF } \\
\text { SAMPLE }\end{array}$ & $\begin{array}{l}\text { SUL IDS, } \\
\text { RESIUUE } \\
\text { AT } 180 \\
\text { OEG.C } \\
\text { OIS- } \\
\text { SOLVED } \\
\text { (MG/L) }\end{array}$ & $\begin{array}{c}\text { BICAR- } \\
\text { BONATE } \\
\text { (MG/L } \\
\text { AS } \\
\text { HCO3) }\end{array}$ & $\begin{array}{l}\text { CAR- } \\
\text { BUNATE } \\
\text { (MG/L } \\
\text { AS CO3) }\end{array}$ & $\begin{array}{l}\text { HARO- } \\
\text { NESS } \\
\text { (MG/L } \\
\text { AS } \\
\text { CACO3) }\end{array}$ & $\begin{array}{l}\text { HARU- } \\
\text { NESS, } \\
\text { NONCAR- } \\
\text { BONATE } \\
\text { (MG/L } \\
\text { CACU3) }\end{array}$ & $\begin{array}{l}\text { SULFATE } \\
\text { DIS- } \\
\text { SOLVED } \\
\text { (MG/L } \\
\text { AS SO4) }\end{array}$ & $\begin{array}{l}\text { FLUO- } \\
\text { RIDE, } \\
\text { DIS- } \\
\text { SOLVEO } \\
\text { (MG/L } \\
\text { AS F) }\end{array}$ & $\begin{array}{l}\text { SILICA, } \\
\text { DIS- } \\
\text { SOLVED } \\
\text { (MGLL } \\
\text { AS } \\
\text { SIOE) }\end{array}$ & $\begin{array}{c}\text { CARBON } \\
\text { UIOXIDE } \\
\text { DIS- } \\
\text { SOLVEO } \\
\text { IMG/L } \\
\text { AS COZ) }\end{array}$ \\
\hline 280248082340401 & $\begin{array}{l}73-08-13 \\
73-12-19\end{array}$ & $=$ & $=$ & $=$ & $\begin{array}{l}19 \\
18\end{array}$ & $=$ & $=$ & $=$ & $=$ & $=$ \\
\hline 280249082340501 & $72-07-17$ & -- & -- & - & 200 & - & -- & -- & -- & -- \\
\hline
\end{tabular}


Table 7, --Nitrogen and phosphorus data for ground-water sites at the Rocky Creek landfill

\begin{tabular}{|c|c|c|c|c|c|c|c|c|c|c|c|}
\hline STATION NUMBER & $\begin{array}{l}\text { OATE } \\
\text { OF } \\
\text { SAMPLE }\end{array}$ & $\begin{array}{l}\text { NITRO- } \\
\text { GEN, } \\
\text { ORGANIC } \\
\text { TOTAL } \\
\text { (MG/L } \\
\text { AS N) }\end{array}$ & $\begin{array}{l}\text { NITRU- } \\
\text { GEN, } \\
\text { ORGANIC } \\
\text { DIS- } \\
\text { SOLVED } \\
\text { (MG/L } \\
\text { AS N) }\end{array}$ & $\begin{array}{l}\text { NITRO- } \\
\text { GEN } \\
\text { AMMONIA } \\
\text { OIS- } \\
\text { SOLVED } \\
\text { IMG/L } \\
\text { AS NI }\end{array}$ & $\begin{array}{l}\text { NI IRO- } \\
\text { GEN, } \\
\text { AMMONIA } \\
\text { TOTAL } \\
\text { (MGA } \\
\text { AS N) }\end{array}$ & $\begin{array}{l}\text { NITRO- } \\
\text { GEN } \\
\text { NITITE } \\
\text { UIS- } \\
\text { SOLVEO } \\
\text { (MG/L } \\
\text { AS N) }\end{array}$ & $\begin{array}{l}\text { NITRO- } \\
\text { GEN, } \\
\text { NITRITE } \\
\text { TOTAL } \\
\text { (MGA } \\
\text { AS N) }\end{array}$ & $\begin{array}{l}\text { NIIRO- } \\
\text { GEN } \\
\text { NITRATE } \\
\text { OISS- } \\
\text { SOLVEO } \\
\text { (MG/L } \\
\text { AS N) }\end{array}$ & $\begin{array}{l}\text { NITRO- } \\
\text { GEN. } \\
\text { NITRATE } \\
\text { TOTAL } \\
\text { (MGAL } \\
\text { AS NI }\end{array}$ & $\begin{array}{l}\text { PHOS- } \\
\text { PHORUS, } \\
\text { TOOAL } \\
\text { (MG/ } \\
\text { AS P) }\end{array}$ & $\begin{array}{l}\text { PHOS- } \\
\text { PHOKUS, } \\
\text { OIS- } \\
\text { SOLVED } \\
\text { (MG/L } \\
\text { AS P) }\end{array}$ \\
\hline 280219082342801 & $\begin{array}{l}70-01-29 \\
70-10-23\end{array}$ & $=$ & . 27 & .16 & $=$ & $\begin{array}{l}.00 \\
.00\end{array}$ & $=$ & .02 & $=$ & .080 & $=$ \\
\hline \multirow[t]{2}{*}{280221082342901} & $\begin{array}{l}70-04-20 \\
70-10-23\end{array}$ & .03 & $\because-$ & .13 & $\because-$ & $\begin{array}{l}.01 \\
.00\end{array}$ & $=$ & .00 & $=$ & .060 & $\because$ \\
\hline & $73-04-23$ & .62 & -- & $\cdots$ & .33 & $\cdots$ & .00 & -- & .00 & -- & -- \\
\hline 280226082341202 & $\begin{array}{l}73-04-24 \\
73-04-25 \\
73-04-25\end{array}$ &.-37 & $\because$ & .16 & $\because$ & .00 & .02 & .00 & .10 & $=$ & $\bar{z}$ \\
\hline 280226082341201 & $\begin{array}{l}73-04-25 \\
73-06-07\end{array}$ & 1.8 & $\because$ & .20 & - & .00 & $=$ & .00 & $=$ & 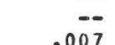 & $=$ \\
\hline \multirow[t]{2}{*}{280227082343001} & $70-01-29$ & .50 & - & .25 & - & .01 & - & .00 & -- & - & -- \\
\hline & $\begin{array}{l}70-10-22 \\
73-01-23 \\
73-03-19 \\
73-04-23\end{array}$ & $\begin{array}{l}-= \\
.04 \\
.22\end{array}$ & $\begin{array}{l}.7 \\
.12 \\
--\end{array}$ & $\begin{array}{r}.- \\
.40 \\
.33 \\
--\end{array}$ & $=-$ & $\begin{array}{l}.00 \\
.00 \\
.00 \\
-0\end{array}$ & $\begin{array}{l}=- \\
=0 \\
.00\end{array}$ & $\begin{array}{r}.0 \\
.00 \\
.00 \\
-0\end{array}$ & $\begin{array}{l}-z \\
.0 \\
.00\end{array}$ & $\begin{array}{r}10 \\
.110 \\
.150 \\
-0\end{array}$ & $\begin{array}{l}= \\
\because \\
=\end{array}$ \\
\hline $\begin{array}{l}2802280823355901 \\
280228082342601\end{array}$ & $70-01-30$ & $\cdots$ & .12 & -- & .00 & .91 & -- & .02 & -- & .010 & \\
\hline \multirow{2}{*}{$\begin{array}{l}280228082342602 \\
280228082342603\end{array}$} & $\begin{array}{l}70-10-23 \\
70-01-30\end{array}$ & $\because$ &.$\ddot{24}$ & .15 & $\because$ & $\begin{array}{l}.00 \\
.01\end{array}$ & $=$ & .00 & $=$ & $.0 \overline{-}$ & $\because$ \\
\hline & $70-01$ & $=$ & .49 & .00 & - & .01 & $=$ & .11 & - & $=$ & $=$ \\
\hline 280228082342701 & $71-10-28$ & - & - & $=$ & $\because$ & .00 & $=$ & .00 & - & - & - \\
\hline 280231082343603 & $70-02-04$ & -- & .31 & .22 & -- & .00 & -- & .00 & -- & .010 & -- \\
\hline \multirow{2}{*}{$\begin{array}{l}280231082343602 \\
280231082343600\end{array}$} & $\begin{array}{l}70-1 \\
70-0\end{array}$ & $=$ & $=$ & 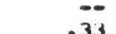 & $=$ & & $=$ & $\ddot{07}$ & $=$ & $\ddot{-a}$ & $=$ \\
\hline & $70-06-15$ & - & 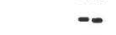 & $\cdots$ & 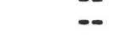 & 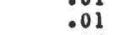 & -0 & -- & -. & - & \\
\hline 280231082343601 & $70-02-04$ & .55 & -- & .20 & -- & .02 & -- & -- & -- & .007 & -- \\
\hline \multirow{3}{*}{$\begin{array}{l}280231082342602 \\
280231082342601 \\
280233062340702\end{array}$} & $\begin{array}{l}73-01-23 \\
70-01-30\end{array}$ & $=$ & $\begin{array}{l}.12 \\
.32\end{array}$ & .41 & $=$ & .00 & $=$ & .00 & $=-$ & .200 & $=$ \\
\hline & $70-0$ & $=-$ &.$<2$ & .11 & -- & .00 & - & .11 & -- & .007 & - \\
\hline & $\begin{array}{l}73-04-25 \\
73-10-03\end{array}$ & $\begin{array}{l}.34 \\
.68\end{array}$ & $=$ & .018 & $\because$ & $\begin{array}{l}.00 \\
.00\end{array}$ & $\because$ & .00 & .00 & $=$ & $=$ \\
\hline \multirow[t]{2}{*}{$\begin{array}{l}280233082340701 \\
280236082343902\end{array}$} & $\begin{array}{l}73-10-25 \\
70-02-04\end{array}$ & $\begin{array}{l}1.3 \\
.29\end{array}$ & $=$ & $\begin{array}{l}1.0 \\
.10\end{array}$ & $=$ & $\begin{array}{l}.01 \\
.00\end{array}$ & $\because$ & $\begin{array}{l}.00 \\
.00\end{array}$ & $=$ & .000 & $=$ \\
\hline & & $=$ & - & $=$ & $=$ & .0 0 & $=$ & $=$ & $=$ & $=$ & $=$ \\
\hline $\begin{array}{l}280233082342302 \\
280233082342602\end{array}$ & 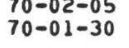 & $\because$ & .54 &.$\overline{26}$ & $=$ & .0 & $=$ & $\because$ & $\because-$ & .010 & $=$ \\
\hline \multirow{3}{*}{$\begin{array}{l}280235082335801 \\
280235082341701\end{array}$} & $\begin{array}{l}70-10-23 \\
73-04-26\end{array}$ & $=$ & $\because$ & $=$ & $=$ & .00 &.$\overline{19}$ & $=$ & .00 & $=$ & $=$ \\
\hline & & 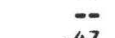 & $\because$ & .36 & -- & .00 & $-\infty$ & .00 & -- & -- & -- \\
\hline & $\begin{array}{l}73-04-25 \\
73-08-10\end{array}$ & $\begin{array}{l}.47 \\
.42\end{array}$ & $=$ & $\begin{array}{l}.03 \\
.14\end{array}$ & $\because$ & .00 & $=$ & .000 & $=$ & .230 & $=$ \\
\hline \multirow[t]{2}{*}{280237082343801} & 70 & -- & .57 & .13 & -- & .01 & -- & .00 & -- & $\cdots$ & $=$ \\
\hline & & -- & $=$ & $\because$ & $\because$ & $\because$ & -- & .27 & $=-$ & $=$ & $=$ \\
\hline 280237082343802 & $\begin{array}{l}70-02-04 \\
73-04-24\end{array}$ & $=$ & .32 & .02 & $=$ & .02 & $=$ & .00 & .10 & .007 & $=$ \\
\hline \multirow{3}{*}{$\begin{array}{l}2802370823438003 \\
280238082340902 \\
280238082340901\end{array}$} & 70. & -- & .22 & .05 & -- & .00 & -- & .00 & -- & -- & $\cdots$ \\
\hline & & 1.9 & $=$ & $\begin{array}{r}.46 \\
.39\end{array}$ & -- & .0 & $=$ & .00 & $=$ & $\begin{array}{l}900 \\
.000\end{array}$ & $=$ \\
\hline & $\begin{array}{l}73-10-02 \\
73-10-24\end{array}$ & 3 & $=$ & .41 & -- & 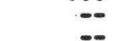 & $=$ & $-\overline{0}$ & $=-$ & $=$ & $=$ \\
\hline \multirow{4}{*}{$\begin{array}{r}280238082343202 \\
280238082343201\end{array}$} & 73 & .30 & -. & .34 & -- & .00 & -- & .00 & -- & -- & .011 \\
\hline & & .33 & $\ddot{m}$ & 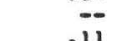 & .44 & & .00 & & .01 & & -- \\
\hline & $\begin{array}{l}70=02- \\
70=02-\end{array}$ & $=$ & .46 & 11 & $\because$ & .011 & $=$ & 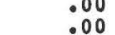 & $=$ & .0107 & $=$ \\
\hline & $70-02-$ & .55 & -- & .10 & -- & - & -- & .00 & -- & .010 & -- \\
\hline \multirow{3}{*}{280238082342602} & $\begin{array}{l}70-10-22 \\
73-02-07\end{array}$ &.$\overline{06}$ & $=$ &.$\ddot{36}$ & $=$ & $\begin{array}{l}.00 \\
.00\end{array}$ & $=$ & .00 & $=$ & $=$ & $=$ \\
\hline & & .58 & $\ddot{a}$ & & -- & & -- & & -- & -- & -- \\
\hline & $\begin{array}{l}70-02-02 \\
70-10-22\end{array}$ & $=$ & .31 & 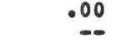 & $\because$ & .00 & $=$ & .00 & $=$ & $=$ & $=$ \\
\hline \multirow{2}{*}{$\begin{array}{l}280238082342601 \\
280238082335701\end{array}$} & $70-02-02$ & $\cdots$ & .36 & .00 & -- & .00 & $\cdots$ & .00 & $=-$ & .200 & $=-$ \\
\hline & & $\cdots$ & -- & - & -- & $\cdots$ & .00 & -- & .00 & -- & $=$ \\
\hline 280240082340601 & $\begin{array}{l}73-10-24 \\
73-04-23\end{array}$ & $\begin{array}{l}1.0 \\
.39\end{array}$ & $=$ & $=$ & $\begin{array}{l}.20 \\
.29\end{array}$ & $\because$ & .00 & $\because$ & $\begin{array}{l}.00 \\
00\end{array}$ & $=$ & $\ldots$ \\
\hline & $73-06-07$ & .51 & -- & 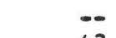 & .24 & 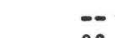 & .00 & $-\bar{x}+2+$ & .00 & $\ddot{0}$ & $\overline{-}$ \\
\hline 280240082341202 & & $\begin{array}{r}.50 \\
.06\end{array}$ & $\because$ & 3 & 44 & 00 &.$\overline{00}$ & 00 & .00 & .020 & $\ldots$ \\
\hline 280240082341201 & & 1.0 & -- & .44 & -- & - & $=$ & .00 & - & .000 & -- \\
\hline & $73-10=$ & & & & & & & & & & \\
\hline & $\begin{array}{l}73-10-24 \\
73-11-27\end{array}$ & $\begin{array}{l}.73 \\
.96\end{array}$ & $=$ & $\begin{array}{l}.28 \\
.69\end{array}$ & $=$ & .011 & $=$ & $\begin{array}{l}.00 \\
.00\end{array}$ & $=$ & .780 & 130 \\
\hline 280239082343502 & & . & .27 & & -- & & -- & & -- & .030 & $=$ \\
\hline & $\begin{array}{l}73-02-07 \\
73-02-26\end{array}$ & 2.8 & .00 & 4 & $\because$ & & $=$ & $\begin{array}{l}.00 \\
.00\end{array}$ & $\because$ & $\because$ & $\cdots$ \\
\hline & $3-03-1$ & .88 & -- & .1 & -- & .0 & -- & .00 & -- & .050 & -- \\
\hline & & .9 & $=$ & $\begin{array}{l}.49 \\
.39\end{array}$ & $=$ & : & $=$ & & $=$ & & \\
\hline 280239082343501 & 73 & .71 & -- & $\cdots$ & .40 & 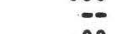 & .00 & 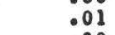 & -- & .110 & - \\
\hline & $73-02-26$ & .16 & -- & $\cdots$ & .53 & & & & -- & & \\
\hline
\end{tabular}


Table 7.--Nitrogen and phosphorus data for ground-water sites at the Rocky Creek landfill--Continued

\begin{tabular}{|c|c|c|c|c|c|c|c|c|c|c|c|}
\hline STATION NUMBER & $\begin{array}{c}\text { DATE } \\
\text { OF } \\
\text { SAMPLE }\end{array}$ & $\begin{array}{l}\text { NITKO- } \\
\text { GEN, } \\
\text { ORGANIC } \\
\text { TOTAL } \\
\text { (MG/L } \\
\text { AS N) }\end{array}$ & $\begin{array}{l}\text { NITRU- } \\
\text { GEN, } \\
\text { ORGANIC } \\
\text { DIS- } \\
\text { SOLVEO } \\
\text { (MG/L } \\
\text { AS N) }\end{array}$ & $\begin{array}{l}\text { NITRO- } \\
\text { GEN, } \\
\text { AMMONIA } \\
\text { DIS- } \\
\text { SOLVEU } \\
\text { (MG } / L \\
\text { AS N) }\end{array}$ & $\begin{array}{l}\text { NI TRO- } \\
\text { GEN, } \\
\text { AMMONIA } \\
\text { TOTAL } \\
\text { (MG/L } \\
\text { AS N) }\end{array}$ & $\begin{array}{l}\text { NITRO- } \\
\text { GEN, } \\
\text { NITRITE } \\
\text { OIS- } \\
\text { SOLVED } \\
\text { (MG/L } \\
\text { AS N) }\end{array}$ & $\begin{array}{l}\text { NITRO- } \\
\text { GEN, } \\
\text { NITRITE } \\
\text { TOTALL } \\
\text { (MGLL } \\
\text { AS N) }\end{array}$ & $\begin{array}{l}\text { NITRO- } \\
\text { GEN, } \\
\text { NITRATE } \\
\text { DIS- } \\
\text { SOLVEO } \\
\text { (MG/L } \\
\text { AS N) }\end{array}$ & $\begin{array}{l}\text { NITRO- } \\
\text { GEN, } \\
\text { NITRATE } \\
\text { TOTAL } \\
\text { (MG/L } \\
\text { AS N) }\end{array}$ & $\begin{array}{l}\text { PHOS- } \\
\text { PHORUS, } \\
\text { TOTAL } \\
\text { (MG/L } \\
\text { AS P) }\end{array}$ & $\begin{array}{l}\text { PHOS- } \\
\text { PHORUS, } \\
\text { OIS- } \\
\text { SOLVED } \\
\text { IMG/L } \\
\text { AS P) }\end{array}$ \\
\hline \multirow[t]{2}{*}{280239082343501} & $\begin{array}{l}73-03-19 \\
73-04-24\end{array}$ & $\begin{array}{l}.08 \\
.24\end{array}$ & $=$ & $=$ & .33 & $\begin{array}{l}.00 \\
.00\end{array}$ & .00 & $\begin{array}{l}.00 \\
.18\end{array}$ & $=$ & $=$ & $=$ \\
\hline & $73-06-07$ & .20 & -- & -- & .50 & -- & .00 & -. & .00 & -- & -- \\
\hline \multirow[t]{2}{*}{280240082342601} & $73-02-26$ & 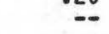 & -- & .27 & $\cdots$ & .00 & 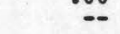 & .00 & - & -- & -- \\
\hline & $73-03-19$ & - & -- & .28 & - & .00 & -- & .00 & -- & .110 & - \\
\hline \multirow[t]{7}{*}{280242082343502} & $73-01-23$ & -- & .60 & .14 & - & .01 & -- & .00 & -- & .150 & -- \\
\hline & $73-02-26$ & -- & .11 & 1.4 & -- & .03 & -- & .00 & -- & 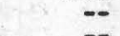 & 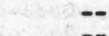 \\
\hline & $73-04-24$ & 1.1 & $=-$ & .82 & -- & .00 & -- & .01 & $=$ & $-\bar{x}$ & - \\
\hline & $73-06-07$ & $=$ & $\overline{4}$ & $\overline{17}$ & $=$ & .00 & $=$ & .01 & $=$ & .006 & $=$ \\
\hline & $73-10-02$ & $\cdots$ & & -11 & $\cdots$ & .00 & $\cdots$ & .00 & $\cdots$ & .030 & \\
\hline & $73-10-24$ & $=$ & .43 & .00 & - & -- & .00 & - & .00 & .030 & 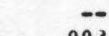 \\
\hline & $\begin{array}{l}73-11-27 \\
73-12-18\end{array}$ & .70 & $=$ & .38 & $=$ & .00 & $=$ & .02 & $=-$ & $\begin{array}{r}.260 \\
.620\end{array}$ & .003 \\
\hline \multirow[t]{7}{*}{280242082343501} & $72-06-06$ & - & -- & 1.0 & -- & - & -- & - & -- & $\ldots$ & - \\
\hline & $73-01-23$ & $\cdots$ & .16 & .48 & $\cdots$ & .00 & -- & .00 & -- & .070 & - \\
\hline & $73-02-26$ & .26 & -- & .42 & -- & .00 & -- & .00 & - & - & -- \\
\hline & $73-04-24$ & 1.2 & -- & .47 & -- & .00 & -- & .04 & -- & - & - \\
\hline & $73-10-02$ & - & .29 & .45 & -- & .00 & -- & .00 & - & .080 & -- \\
\hline & 73 & .26 & -- & -- & .39 & .0 & -- & -- & .00 & -- & -- \\
\hline & $73-11-27$ & .16 & $\cdots$ & .48 & - & .00 & -- & .00 & -- & .340 & .074 \\
\hline \multirow{5}{*}{$\begin{array}{l}280245082342701 \\
280242082342601\end{array}$} & $73-12-18$ & .10 & - & -- & .55 & - & .00 & - & .00 & .260 & -- \\
\hline & $71-10-28$ & -- & -- & -- & -- & .12 & - & .00 & -- & & -- \\
\hline & $73-04-23$ & .26 & $-\infty$ & .00 & -- & .00 & $=-$ & .04 & -- & -- & -- \\
\hline & $73-08-10$ & .63 & $-\infty$ & .43 & -- & .00 & .01 & .00 & - & .050 & -- \\
\hline & $73-10-02$ & $-x^{-}$ & .63 & .43 & -- & .01 & -- & .00 & -- & .020 & -- \\
\hline \multirow{2}{*}{280242082342602} & $73-04-23$ & 1.7 & -- & .87 & - & .00 & -- & .01 & -- & -- & -- \\
\hline & $73-10-02$ & .15 & -- & .15 & -- & .03 & -- & .00 & -- & .030 & $n_{0}$ \\
\hline \multirow{2}{*}{280246082343202} & $70-10-22$ & -- & -- & -- & - & .00 & -- & -- & - & -- & - \\
\hline & $73-c$ & 3.9 & - & .21 & - & .0 & -- & .00 & $-x_{-1}$ & 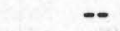 & $=$ \\
\hline 280243082342601 & $70-10-22$ & -- & -- & -- & -- & .00 & -- & -- & -- & -- & -- \\
\hline
\end{tabular}


Table 8, --Trace-metals data for ground-water sites at the Rocky Creek landfill

\begin{tabular}{|c|c|c|c|c|c|c|c|c|c|c|}
\hline STATION NUMBER & $\begin{array}{c}\text { DATE } \\
\text { OF } \\
\text { SAMPLEE }\end{array}$ & $\begin{array}{l}\text { ARSENIC } \\
\text { OIS- } \\
\text { SOLVEO } \\
\text { UG AL } \\
\text { AS AS }\end{array}$ & $\begin{array}{l}\text { CHRO- } \\
\text { MIUM, } \\
\text { OIS- } \\
\text { SOLVED } \\
\text { (UG/L } \\
\text { AS CK) }\end{array}$ & $\begin{array}{l}\text { COPPER, } \\
\text { OIS- } \\
\text { SOLVED } \\
\text { UG AL } \\
\text { AS CU) }\end{array}$ & $\begin{array}{l}\text { IRON. } \\
\text { TOTAL } \\
\text { RECOV- } \\
\text { ERABLE } \\
\text { (UG/L } \\
\text { AS FE) }\end{array}$ & $\begin{array}{l}\text { LEAU, } \\
\text { DIS- } \\
\text { SOLVED } \\
\text { (UG/L } \\
\text { AS PB) }\end{array}$ & $\begin{array}{l}\text { MANGA- } \\
\text { NESE, } \\
\text { TOTAL } \\
\text { RECOV- } \\
\text { EWAGLE } \\
\text { ING/L } \\
\text { AS MNI }\end{array}$ & $\begin{array}{l}\text { STRON- } \\
\text { TIUM, } \\
\text { OIS- } \\
\text { SOLVEO } \\
\text { IUG/L } \\
\text { AS SRI }\end{array}$ & $\begin{array}{l}\text { ZINC. } \\
\text { OIS- } \\
\text { SULVED } \\
\text { (UGRL } \\
\text { AS ZN) }\end{array}$ & $\begin{array}{l}\text { ALUM- } \\
\text { INUM, } \\
\text { OIS- } \\
\text { SOLVED } \\
\text { (UGAL } \\
\text { AS AL) }\end{array}$ \\
\hline $\begin{array}{l}280219082342801 \\
280221082342901\end{array}$ & $\begin{array}{l}70-01-29 \\
70-10-23 \\
70-011-29 \\
70-10-23 \\
70-10-27\end{array}$ & $\begin{array}{r}10 \\
10 \\
0 \\
0 \\
\therefore\end{array}$ & $\begin{array}{r}0 \\
0 \\
10 \\
0 \\
--\end{array}$ & $\begin{array}{l}0 \\
0 \\
0 \\
0 \\
\therefore\end{array}$ & $\begin{array}{l}140 \\
300 \\
510 \\
460 \\
600\end{array}$ & $\begin{array}{l}0 \\
0 \\
0 \\
0 \\
\therefore\end{array}$ & $\begin{array}{l}10 \\
10 \\
20 \\
0 \\
-\end{array}$ & $\begin{array}{r}100 \\
140 \\
30 \\
10 \\
--\end{array}$ & $\begin{array}{r}80 \\
260 \\
190 \\
100 \\
-\end{array}$ & $\begin{array}{l}-8 \\
30 \\
--\end{array}$ \\
\hline $\begin{array}{l}280226082340701 \\
280226082342101\end{array}$ & $\begin{array}{l}71-12-27 \\
70-01-29 \\
70-01-29 \\
70-033-30 \\
70-04-20\end{array}$ & $\begin{array}{l}\because \\
\because \\
\because\end{array}$ & $\begin{array}{l}\because \\
\because \\
\because\end{array}$ & $\begin{array}{l}= \\
\because \\
\therefore\end{array}$ & $\begin{array}{r}1500 \\
0 \\
100 \\
2400 \\
1200\end{array}$ & $\begin{array}{l}\because \\
\because \\
= \\
=\end{array}$ & 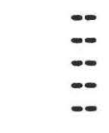 & $\begin{array}{l}= \\
=- \\
=\end{array}$ & $\begin{array}{l}= \\
= \\
=\end{array}$ & $\begin{array}{l}= \\
= \\
=\end{array}$ \\
\hline & $\begin{array}{l}70-09-18 \\
70-10-27 \\
70-12-03 \\
71-01-14 \\
71=03-31\end{array}$ & $\begin{array}{l}\because \\
\because \\
= \\
=\end{array}$ & $\begin{array}{l}= \\
=- \\
=\end{array}$ & $\begin{array}{l}\because \\
\because \\
\because \\
=\end{array}$ & $\begin{array}{r}5000 \\
5300 \\
1330 \\
8500 \\
26000\end{array}$ & $\begin{array}{l}\because \\
\because \\
= \\
=\end{array}$ & 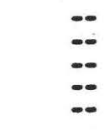 & $\begin{array}{l}= \\
= \\
= \\
=\end{array}$ & $\begin{array}{l}\because \\
\because \\
\because\end{array}$ & $\begin{array}{l}= \\
=- \\
=-\end{array}$ \\
\hline 280226082342502 & $\begin{array}{l}71-04-28 \\
71-06-15 \\
71-07-28 \\
71-10-29 \\
70-01-29\end{array}$ & $\begin{array}{l}\ddot{z} \\
\ddot{z}\end{array}$ & $\begin{array}{l}\because \\
\because \\
\because\end{array}$ & $\begin{array}{l}\because \\
\because \\
\therefore \\
=\end{array}$ & $\begin{array}{l}52000 \\
7800 \\
28000 \\
12000 \\
0\end{array}$ & $\begin{array}{l}\because \\
\because \\
\because\end{array}$ & $\begin{array}{l}\because \\
\because \\
\because\end{array}$ & $\begin{array}{l}\because \\
\because \\
\because\end{array}$ & $\begin{array}{l}\because \\
\because \\
\ddot{z}\end{array}$ & $\begin{array}{l}\because \\
\because \because \\
\ddot{z}\end{array}$ \\
\hline & $\begin{array}{l}70-04-20 \\
70-10-26 \\
70-12-03 \\
71-01-14 \\
71-03-30\end{array}$ & $\begin{array}{l}\because \\
\ddot{z}\end{array}$ & $\begin{array}{l}\because \\
\because \\
\because\end{array}$ & $\begin{array}{l}\because \\
\because \\
\because\end{array}$ & $\begin{array}{r}940 \\
1200 \\
260 \\
2000 \\
1300\end{array}$ & $\begin{array}{l}\because \\
\because \because \\
\because\end{array}$ & $\begin{array}{l}\because \\
\because \\
=\end{array}$ & $\begin{array}{l}\because \\
\because \\
\because\end{array}$ & $\begin{array}{l}\because \\
\because \\
\because\end{array}$ & $\begin{array}{l}\square \\
\because \\
z\end{array}$ \\
\hline 280226082342902 & $\begin{array}{l}71-04-28 \\
71-07-27 \\
71-10-29 \\
72-010-27 \\
70-03-30\end{array}$ & $\begin{array}{l}\because \\
\because \\
=\end{array}$ & $\begin{array}{l}\ddot{z} \\
\because= \\
=\end{array}$ & $\begin{array}{l}= \\
\because \\
=\end{array}$ & $\begin{array}{r}5700 \\
2600 \\
1400 \\
1500 \\
11000\end{array}$ & $\begin{array}{l}\because \\
\because \\
\because\end{array}$ & $\begin{array}{l}= \\
= \\
=\end{array}$ & $\begin{array}{l}\because \\
\because \\
\because\end{array}$ & 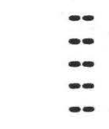 & $\begin{array}{l}\square \\
\because \\
z\end{array}$ \\
\hline 280227082343001 & $\begin{array}{l}70-01-29 \\
70-03-30 \\
70-04-20 \\
70-09-18 \\
70-10-22\end{array}$ & $\begin{array}{l}0 \\
\because \because \\
20\end{array}$ & $\begin{array}{l}10 \\
\because \\
\because 0\end{array}$ & $\begin{array}{l}10 \\
\because \because \\
\because 0\end{array}$ & $\begin{array}{r}50 \\
0 \\
40 \\
800 \\
100\end{array}$ & $\begin{array}{l}0 \\
\because \because \\
0\end{array}$ & $\begin{array}{l}10 \\
=- \\
10\end{array}$ & $\begin{array}{l}80 \\
\because \because \\
130\end{array}$ & $\begin{array}{l}40 \\
\because \\
\square 0\end{array}$ & $\begin{array}{l}\because \\
\because \\
\ddot{110}\end{array}$ \\
\hline & $\begin{array}{l}70-12-03 \\
71-01-14 \\
71-020-24 \\
71-03-31 \\
71-04-28\end{array}$ & $\begin{array}{l}\because \\
\because \\
\because\end{array}$ & $\begin{array}{l}\because \\
\because \\
\because\end{array}$ & $\begin{array}{l}\because \\
\because \\
\because\end{array}$ & $\begin{array}{r}260 \\
2200 \\
0 \\
590 \\
3600\end{array}$ & $\begin{array}{l}\because \\
\because \\
\because\end{array}$ & $\begin{array}{l}\because \\
\because \\
\because\end{array}$ & $\begin{array}{l}\because \\
\because \because \\
\because\end{array}$ & $\begin{array}{l}\because \\
\because \\
\square\end{array}$ & $\begin{array}{l}\because \\
\because \\
z\end{array}$ \\
\hline $\begin{array}{l}280228082342601 \\
280228082342602\end{array}$ & $\begin{array}{l}71-06-15 \\
71-07-28 \\
70-01-30 \\
70-10-23 \\
70-01-30\end{array}$ & $\begin{array}{l}\because \\
0 \\
0 \\
0\end{array}$ & $\begin{array}{l}\ddot{0} \\
\ddot{10}\end{array}$ & $\begin{array}{l}\because \\
0 \\
0 \\
10\end{array}$ & $\begin{array}{r}980 \\
270 \\
60 \\
1400\end{array}$ & $\begin{array}{l}\because \\
0 \\
10 \\
0\end{array}$ & $\begin{array}{l}\ddot{0} \\
80 \\
20\end{array}$ & $\begin{array}{l}\because z \\
120 \\
140 \\
50\end{array}$ & $\begin{array}{r}\because z \\
250 \\
60 \\
4700\end{array}$ & $\begin{array}{l}\because \\
\because 8 \\
60\end{array}$ \\
\hline & $\begin{array}{l}70-03-30 \\
70-04-20 \\
70-08-05 \\
70-09-18 \\
710-09-18 \\
71-02-26\end{array}$ & $\begin{array}{l}\because \\
\because \\
\because\end{array}$ & $=$ & $\begin{array}{l}\because \\
\because \\
\because\end{array}$ & $\begin{array}{r}11000 \\
6500 \\
2330 \\
6100 \\
1930\end{array}$ & $\begin{array}{l}\because \\
\because \\
\because\end{array}$ & $\begin{array}{l}\Xi \\
\ddot{\Xi}\end{array}$ & $\begin{array}{l}\ddot{z} \\
\ddot{z}\end{array}$ & $\begin{array}{l}\because \\
\because \\
\because\end{array}$ & $\begin{array}{l}\because \\
z \\
z\end{array}$ \\
\hline 280228082342603 & $\begin{array}{l}71-10-29 \\
70-010-30 \\
70-03-30 \\
70-08-05 \\
70-09-18\end{array}$ & $\begin{array}{l}\ddot{10} \\
\because \\
\because\end{array}$ & $\begin{array}{l}\because \\
\because \\
\because\end{array}$ & $\begin{array}{l}\because 0 \\
\because \because \\
\because\end{array}$ & $\begin{array}{r}490 \\
500 \\
920 \\
780 \\
1000\end{array}$ & $\begin{array}{l}\because \\
\because \\
\because\end{array}$ & $\begin{array}{l}\ddot{40} \\
\because \\
\because\end{array}$ & $\begin{array}{l}90 \\
90 \\
\ddot{-}\end{array}$ & $\begin{array}{r}12000 \\
\ddot{\square}\end{array}$ & $\begin{array}{l}\because \\
\because \\
\because\end{array}$ \\
\hline & $\begin{array}{l}70-10-23 \\
70-12-04 \\
71-01-13 \\
71-02-26 \\
71-03-30\end{array}$ & $\begin{array}{l}10 \\
\square \\
= \\
=-\end{array}$ & $\begin{array}{l}\because \\
\because= \\
\because\end{array}$ & $\begin{array}{l}0 \\
\because \because \\
\because-\end{array}$ & $\begin{array}{r}500 \\
320 \\
2000 \\
730 \\
1500\end{array}$ & $\begin{array}{l}10 \\
=- \\
=-\end{array}$ & $\begin{array}{l}= \\
= \\
=\end{array}$ & $\begin{array}{l}120 \\
\because= \\
\because-\end{array}$ & $\begin{array}{r}3000 \\
\because \\
= \\
=\end{array}$ & $\begin{array}{l}150 \\
\because \because \\
\because=\end{array}$ \\
\hline 280228082342701 & $\begin{array}{l}71-04-28 \\
71-06-14 \\
71-07-28 \\
71-12-28 \\
71-10-28\end{array}$ & $=$ & $=$ & $\begin{array}{l}\because \\
\because \\
\because\end{array}$ & $\begin{array}{r}3400 \\
1500 \\
730 \\
50 \\
-0\end{array}$ & $\begin{array}{l}= \\
= \\
=\end{array}$ & $\begin{array}{l}\because \\
\because \\
=\end{array}$ & $\begin{array}{l}\because \\
\because \\
\square 60 \\
560\end{array}$ & $\begin{array}{l}= \\
= \\
=\end{array}$ & $\begin{array}{l}\because \\
\because \\
\because\end{array}$ \\
\hline 280231082343603 & $\begin{array}{l}70-02-04 \\
70-03-31 \\
70-04-23 \\
70-10-22 \\
70-12-03\end{array}$ & $\because$ & $=$ & 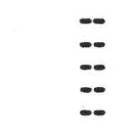 & $\begin{array}{r}520 \\
4800 \\
6500 \\
1470\end{array}$ & $\begin{array}{l}\because \\
\because \\
\because\end{array}$ & $\begin{array}{l}\because \\
\because \\
=\end{array}$ & $\ddot{z}$ & $\begin{array}{l}\because \\
\because \\
\because\end{array}$ & $\begin{array}{l}= \\
\because \\
\because\end{array}$ \\
\hline & $\begin{array}{l}71-01-14 \\
71-02-24 \\
71-03-31 \\
71=04-28 \\
71=06-15\end{array}$ & $\begin{array}{l}\because \\
\because \\
\because\end{array}$ & 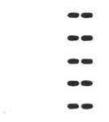 & $\begin{array}{l}\because \\
\because \\
=\end{array}$ & $\begin{array}{l}7500 \\
2240 \\
1200 \\
1200 \\
5000\end{array}$ & $\begin{array}{l}\because \\
\because \\
\because\end{array}$ & $\begin{array}{l}\because \\
\because \\
\because\end{array}$ & 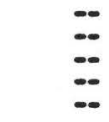 & $\begin{array}{l}\because \\
\ddot{z}\end{array}$ & $\begin{array}{l}\because \\
\because \\
\because\end{array}$ \\
\hline 280231082343602 & $\begin{array}{l}71-07-28 \\
71-10-28 \\
71-12-27 \\
70-02-04 \\
70-06-15\end{array}$ & $\because$ & $=$ & $\begin{array}{l}= \\
\because \\
\because\end{array}$ & $\begin{array}{r}7400 \\
1900 \\
490 \\
\because-\end{array}$ & $\begin{array}{l}\because \\
\because \\
=\end{array}$ & $\begin{array}{l}\because \\
\because \\
\because\end{array}$ & $\begin{array}{l}Z \\
\because 30 \\
30 \\
70\end{array}$ & $\begin{array}{l}\because \\
\because \\
\because\end{array}$ & $\begin{array}{l}\because \\
\because\end{array}$ \\
\hline
\end{tabular}


Table 8. --Trace-metals data for ground-water sites at the Rocky Creek landfill--Continued

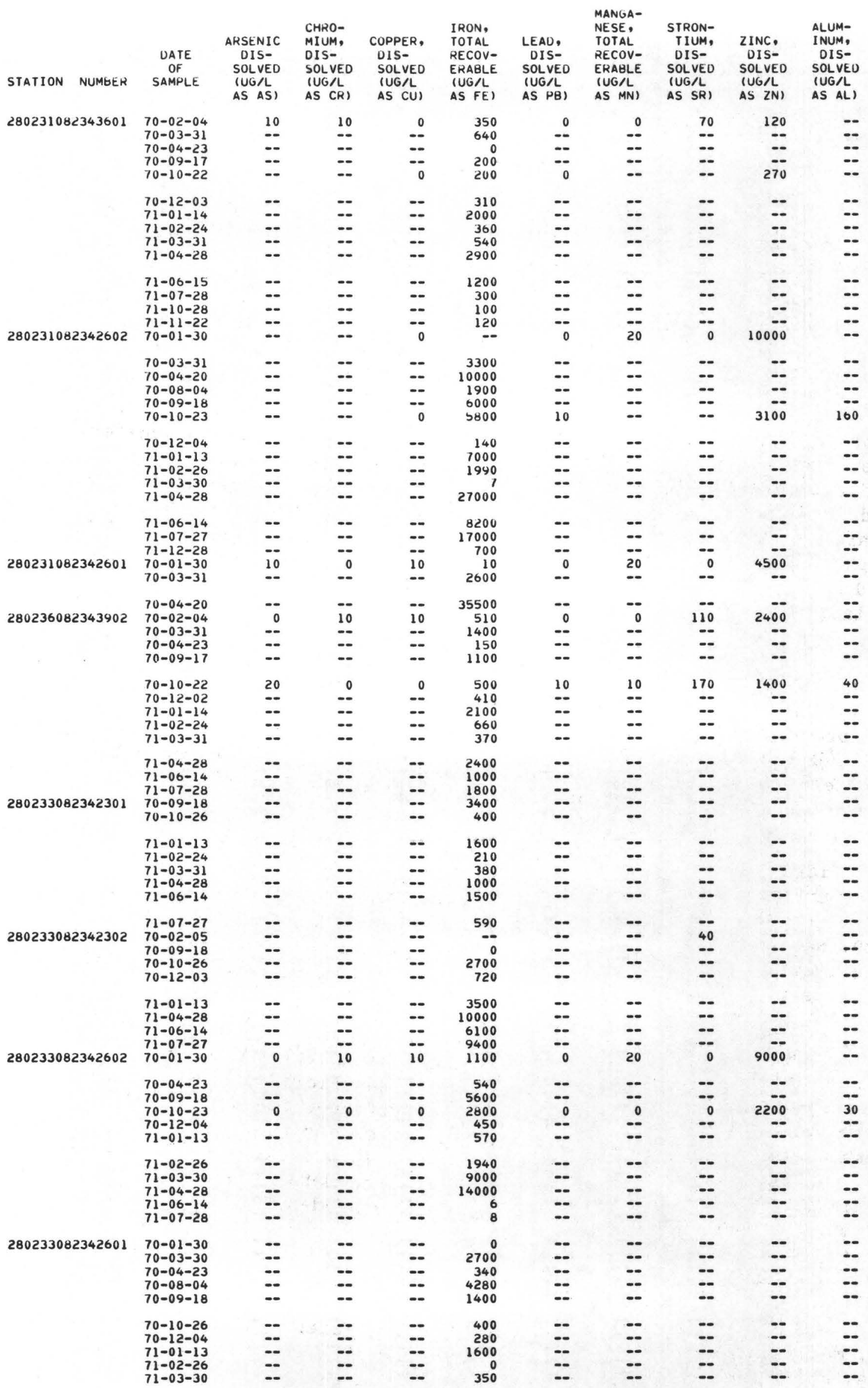


Table 8,--Trace-metals data for ground-water sites at the Rocky CReek Landfill---Continued

\begin{tabular}{|c|c|c|c|c|c|c|c|c|c|c|}
\hline STATION NUMBER & $\begin{array}{c}\text { DATE } \\
\text { OF } \\
\text { SAMPLE }\end{array}$ & $\begin{array}{l}\text { ARSENIC } \\
\text { DIS- } \\
\text { SOLVEU } \\
\text { (UG/L } \\
\text { AS AS) }\end{array}$ & $\begin{array}{l}\text { CHRO- } \\
\text { MIUM, } \\
\text { DIS- } \\
\text { SOLVED } \\
\text { (UG/L } \\
\text { AS CR) }\end{array}$ & $\begin{array}{l}\text { COPPER, } \\
\text { OIS- } \\
\text { SOLVED } \\
\text { (UG/L } \\
\text { AS CU) }\end{array}$ & $\begin{array}{l}\text { IRON, } \\
\text { TOTAL } \\
\text { RECOV- } \\
\text { ERABLE } \\
\text { (UG/L } \\
\text { AS FE) }\end{array}$ & $\begin{array}{l}\text { LEAU, } \\
\text { OIS- } \\
\text { SOLVED } \\
\text { (UG/L } \\
\text { AS PB) }\end{array}$ & $\begin{array}{l}\text { MANUA- } \\
\text { NESE, } \\
\text { TOTAL } \\
\text { RECDV- } \\
\text { ERABLE } \\
\text { (UG/L } \\
\text { AS MN) }\end{array}$ & $\begin{array}{l}\text { STRON- } \\
\text { JIUM, } \\
\text { DIS- } \\
\text { SOLVEO } \\
\text { (UG/L } \\
\text { AS SR) }\end{array}$ & $\begin{array}{l}\text { ZINC, } \\
\text { DIS- } \\
\text { SOLVEO } \\
\text { (UG/L } \\
\text { AS ZN) }\end{array}$ & $\begin{array}{l}\text { ALUM- } \\
\text { INUM, } \\
\text { DIS- } \\
\text { SOLVEO } \\
\text { (UG/L } \\
\text { AS AL) }\end{array}$ \\
\hline 280233082342601 & $71-06-14$ & -- & - & -- & 810 & $\cdots$ & - & -- & -- & -- \\
\hline & $\begin{array}{l}71-07-28 \\
71-10-28\end{array}$ & 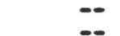 & $=$ & $\overline{-}$ & 820 & - & $=$ & $=$ & $=$ & $=$ \\
\hline 280236082342601 & $\begin{array}{l}1-10-28 \\
72-01-27\end{array}$ & $=$ & $\cdots$ & - & $\begin{array}{r}330 \\
90\end{array}$ & 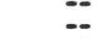 & $=$ & -- & - & $=$ \\
\hline \multirow[t]{3}{*}{280237082343801} & $70-02-04$ & 10 & 10 & 10 & 110 & 0 & 30 & 110 & 300 & -- \\
\hline & $\begin{array}{l}70-10-22 \\
72-07-18\end{array}$ & 30 & $\therefore$ & $\therefore$ & $\begin{array}{r}400 \\
2400\end{array}$ & 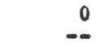 & $\begin{array}{l}0 \\
--\end{array}$ & 160 & 1200 & $=$ \\
\hline & $72-10-25$ & -- & - & - & 4500 & - & - & -- & -- & -- \\
\hline \multirow[t]{2}{*}{280237082343802} & $70-02-04$ & 0 & 10 & 0 & -- & 90 & 10 & 100 & 11000 & - \\
\hline & $70-10-22$ & -- & 0 & 0 & 20 & 0 & 0 & -- & 730 & -- \\
\hline \multirow{5}{*}{$\begin{array}{l}280237082343803 \\
280238082343202\end{array}$} & $70-02-04$ & 10 & 0 & 0 & 840 & 0 & 200 & $0^{0}$ & 2800 & $=$ \\
\hline & $70-02-04$ & 0 & 10 & 0 & 30 & 0 & 10 & 70 & $=$ & $=$ \\
\hline & $\begin{array}{l}70-12-02 \\
71-02-24\end{array}$ & $=$ & $=$ & $=$ & $\begin{array}{l}780 \\
190\end{array}$ & $=$ & 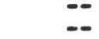 & $=$ & $=$ & $=$ \\
\hline & $71-12-27$ & -- & - & -- & 130 & -- & -- & -- & -- & -- \\
\hline & $\begin{array}{l}72-01-26 \\
72-07-21\end{array}$ & $=$ & $=$ & $=$ & $\begin{array}{l}50 \\
50\end{array}$ & $=$ & $=$ & $=$ & $=$ & $=$ \\
\hline \multirow[t]{3}{*}{280238082343201} & $70-02-02$ & -- & - & - & 0 & 0 & - & 10 & 3500 & -- \\
\hline & $70-02-04$ & 0 & 0 & 0 & 140 & 0 & 20 & 100 & 400 & -- \\
\hline & $70-09-17$ & -- & -- & -- & 2100 & - & -- & -- & -- & -- \\
\hline \multirow{11}{*}{280238082342602} & $70-10-22$ & 20 & 0 & 0 & 10 & 0 & 10 & 150 & 260 & 90 \\
\hline & $70-02-02$ & 10 & 10 & 10 & 130 & 0 & 30 & 0 & 21000 & -- \\
\hline & $70-08-04$ & 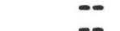 & -- & - & 3170 & - & -- & -- & $=$ & -- \\
\hline & $70-10-22$ & 10 & 0 & 0 & 1000 & 0 & 20 & 20 & 370 & 40 \\
\hline & $70-12-03$ & -- & -- & - & 710 & 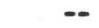 & -- & -- & -- & -- \\
\hline & $71-02-26$ & -- & -- & - & 2110 & $-\infty$ & -- & -- & -- & -- \\
\hline & $71-03-30$ & $=$ & $=$ & -- & 1800 & -- & -- & $=$ & $=$ & $=$ \\
\hline & $71-04-28$ & $\ddot{-}$ & -- & - & 7100 & - & -- & -- & 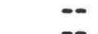 & $=-$ \\
\hline & $11-00-14$ & $\cdots$ & $\cdots$ & $\cdots$ & 9800 & $\cdots$ & $\cdots$ & $\cdots$ & & 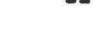 \\
\hline & $71-07-27$ & -- & $\cdots$ & - & 13000 & $\cdots$ & - & -- & $\cdots$ & -- \\
\hline & $\begin{array}{l}72-01-27 \\
72-03-16\end{array}$ & -- & $=$ & -- & 170 & -- & - & - & -- & $=-$ \\
\hline \multirow{11}{*}{280238082342001} & $70-02-02$ & $=$ & $\because$ & $\overline{10}$ & 1700 & 30 & $\ddot{10}$ & 60 & 1100 & 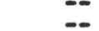 \\
\hline & $70-08-04$ & -- & -- & $\because$ & 2880 & $\ldots$ & 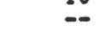 & $\cdots$ & - & -- \\
\hline & $70-09-17$ & - & -- & - & 1800 & - & - & -- & -- & - \\
\hline & $70-10-22$ & 30 & 0 & 0 & 1200 & 0 & 50 & 140 & 100 & 150 \\
\hline & $70-12-03$ & $\cdots$ & $\cdots$ & $\cdots$ & 1590 & $\cdots$ & -- & -- & -- & -- \\
\hline & $71-01-13$ & - & $\cdots$ & $\cdots$ & 2200 & -- & -- & -- & - & -- \\
\hline & $71-02-26$ & - & -- & -- & 720 & - & -- & -- & -- & $\cdots$ \\
\hline & $71-03-30$ & -- & -- & $\cdots$ & 1400 & - & - & -- & -- & -- \\
\hline & $71-04-28$ & - & -- & $\cdots$ & 1600 & $-\infty$ & $\cdots$ & -- & -- & -- \\
\hline & $71-06-14$ & -- & $\cdots$ & $\cdots$ & 1800 & $\cdots$ & -- & $-\infty$ & -- & -- \\
\hline & $71-07-27$ & -- & $\cdots$ & - & 7400 & - & -- & -- & -- & -- \\
\hline 280238082342204 & $70-09-18$ & -- & $\cdots$ & $\cdots$ & 2300 & -- & -- & -- & -- & $\cdots$ \\
\hline & $70-10-26$ & -- & $\cdots$ & $\cdots$ & 500 & $\cdots$ & $\cdots$ & -- & -- & -- \\
\hline & $\begin{array}{l}71-12-03 \\
71-01-13\end{array}$ & $=$ & $=$ & $=$ & $\begin{array}{l}270 \\
4000\end{array}$ & $\ldots$ & $=$ & $\ldots$ & $\ldots$ & $=$ \\
\hline & $71-03-31$ & -- & -- & 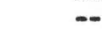 & 580 & - & - & -- & -- & -- \\
\hline & $71-04-28$ & -- & -- & - & 12000 & - & -- & -- & - & -- \\
\hline & $71-06-14$ & -- & -- & - & 1200 & 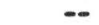 & -- & -- & $\cdots$ & -- \\
\hline & $71-07-2$ & -- & -- & - & 14000 & $\cdots$ & -- & -- & -- & -- \\
\hline 280238082342203 & $70-09-18$ & -- & $\cdots$ & -- & 700 & - & -- & -- & -- & -- \\
\hline & $70-10-26$ & $=$ & $=$ & 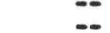 & 400 & $\because$ & $=$ & $\overline{-}$ & $=$ & $=$ \\
\hline & & & & & & & & & & \\
\hline & $71-01-13$ & -- & -- & $\cdots$ & 5700 & $-\infty$ & - & - & -- & -- \\
\hline & $71-02-24$ & 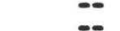 & $=$ & $=$ & 850 & $=$ & $\overline{-}$ & $\overline{-}$ & $\cdots$ & $=-$ \\
\hline & $71=04-28$ & $=$ & $\ldots$ & $\ldots$ & 2300 & $\ldots$ & - & $=$ & $\ldots$ & - \\
\hline & $71-06-14$ & - & $\cdots$ & $\cdots$ & 1400 & $\cdots$ & -- & $-\infty$ & $\cdots$ & -- \\
\hline & $71-07-27$ & -- & -- & $\ldots$ & 1000 & -- & - & -- & - & - \\
\hline & $71-10-29$ & $\cdots$ & -- & - & 160 & - & $\cdots$ & - & $\cdots$ & -- \\
\hline & $72-01$ & $\because$ & $\cdots$ & $\cdots$ & 20 & $\cdots$ & $\cdots$ & $\cdots$ & $\cdots$ & $\cdots$ \\
\hline 280240082340601 & $71-12-28$ & - & -- & 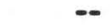 & 40 & $-\infty$ & -- & -- & $\cdots$ & - \\
\hline & $72-01-26$ & -- & -- & - & 50 & - & -- & $\cdots$ & -- & -- \\
\hline 280242082343503 & $70-02-06$ & -- & $\cdots$ & 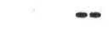 & 4200 & 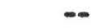 & $\cdots$ & -- & -- & - \\
\hline & $72-01-26$ & - & 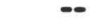 & $\cdots$ & 50 & $\ldots$ & - & -5 & -- & -- \\
\hline & $72-07-20$ & 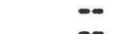 & $=$ & - & 50 & $\cdots$ & $=$ & $=$ & 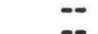 & $=$ \\
\hline 280242082343501 & $70-02-06$ & - & $\cdots$ & $\cdots$ & 100 & -- & $=$ & -- & $=$ & -- \\
\hline & $71-03-31$ & -- & -- & - & 320 & - & -- & -- & -- & -- \\
\hline & $71-04-28$ & -- & -- & $\cdots$ & 1400 & - & -- & -- & -- & -- \\
\hline & $71-06-15$ & $=$ & 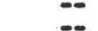 & $\cdots$ & 1000 & - & $\cdots$ & -- & -- & $\overline{-}$ \\
\hline & $\begin{aligned} 71-07-28 \\
71-10-28\end{aligned}$ & $\ddot{z}$ & $=$ & $=$ & 390 & $=$ & $\overline{-}$ & $=$ & $=$ & \\
\hline & 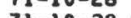 & $=$ & $\ldots$ & $\infty$ & 50 & $\cdots$ & 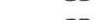 & 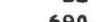 & - & \\
\hline 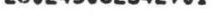 & & & & & 20 & - & & 0 & & \\
\hline 1 & $\begin{array}{l}72-01-27 \\
700-02-02\end{array}$ & $\ddot{m}$ & $=$ & $=$ & 62 & $=$ & $=$ & $=$ & 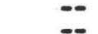 & $=$ \\
\hline 280242082342601 & $\begin{array}{l}70-02-02 \\
70-00-17\end{array}$ & $=$ & 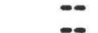 & $=$ & 20 & $=$ & 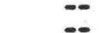 & $\overline{-}$ & $\ddot{m}+$ & $=$ \\
\hline & $70-10-26$ & -- & $\ldots$ & $=$ & 600 & $\ldots$ & $\ldots$ & & - & \\
\hline & $70-12-03$ & - & -- & 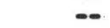 & 1230 & 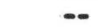 & 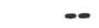 & -- & -- & - \\
\hline
\end{tabular}


Table 8,--Trace-metals data for ground-water sites at the Rocky CReek landfill--Continued

\begin{tabular}{|c|c|c|c|c|c|c|c|c|c|c|}
\hline STATION NUMBER & $\begin{array}{c}\text { DATE } \\
\text { OF } \\
\text { SAMPLE }\end{array}$ & $\begin{array}{c}\text { ARSENIC } \\
\text { OIS- } \\
\text { SOLVEO } \\
\text { (UGAL } \\
\text { AS ASI }\end{array}$ & $\begin{array}{l}\text { CHRO- } \\
\text { MIUM, } \\
\text { OIS- } \\
\text { SOLVED } \\
\text { (UGRL } \\
\text { AS CR) }\end{array}$ & $\begin{array}{l}\text { COPPER, } \\
\text { OIS- } \\
\text { SOLVEU } \\
\text { (UG/L } \\
\text { AS CUI }\end{array}$ & $\begin{array}{l}\text { IRON, } \\
\text { TOTAL } \\
\text { KECOV- } \\
\text { ERABLE } \\
\text { (UG/L } \\
\text { AS FE) }\end{array}$ & $\begin{array}{l}\text { LEAD, } \\
\text { OIS- } \\
\text { SOLVED } \\
\text { (UG/L } \\
\text { AS PB) }\end{array}$ & $\begin{array}{l}\text { MANGA- } \\
\text { NESE, } \\
\text { TOTAL } \\
\text { RECOV- } \\
\text { ERABLE } \\
\text { (UG/L } \\
\text { AS MN) }\end{array}$ & $\begin{array}{l}\text { STRON- } \\
\text { TIUM, } \\
\text { OIS- } \\
\text { SOLVED } \\
\text { IUG/L } \\
\text { AS SRI }\end{array}$ & $\begin{array}{l}\text { ZINC, } \\
\text { DIS- } \\
\text { SOL VEO } \\
\text { (UG/L } \\
\text { AS ZN) }\end{array}$ & $\begin{array}{l}\text { ALUM- } \\
\text { INUM, } \\
\text { DIS- } \\
\text { SOLVED } \\
\text { (UG/L } \\
\text { AS AL) }\end{array}$ \\
\hline \multirow[t]{2}{*}{280242082342601} & $\begin{array}{l}71-01-13 \\
71-02-26 \\
71-03-30 \\
71-04-28 \\
71-06-14\end{array}$ & 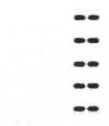 & 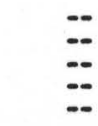 & $\begin{array}{l}= \\
= \\
= \\
-\end{array}$ & $\begin{array}{r}1300 \\
630 \\
450 \\
2900 \\
1200\end{array}$ & $\begin{array}{l}= \\
\because \\
= \\
=\end{array}$ & $\begin{array}{l}= \\
= \\
=\end{array}$ & $\begin{array}{l}= \\
= \\
=\end{array}$ & $\begin{array}{l}\square \\
\therefore \\
=\end{array}$ & 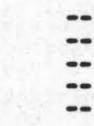 \\
\hline & $\begin{array}{l}71-07-27 \\
71-10-28 \\
71-12-27 \\
72-01-27 \\
72-07-20\end{array}$ & $\begin{array}{l}= \\
=- \\
=\end{array}$ & 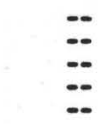 & $\begin{array}{l}= \\
=- \\
--\end{array}$ & $\begin{array}{r}4200 \\
140 \\
120 \\
190 \\
190\end{array}$ & $\begin{array}{l}= \\
= \\
=\end{array}$ & $\begin{array}{l}= \\
= \\
=\end{array}$ & $\begin{array}{l}= \\
\square \\
= \\
=\end{array}$ & $\begin{array}{l}= \\
= \\
=\end{array}$ & $=$ \\
\hline 280242042342602 & $\begin{array}{l}70-02-02 \\
70-09-17 \\
70-10-26 \\
71-07-27 \\
71-10-28\end{array}$ & $\begin{array}{l}= \\
\ddot{-} \\
=-\end{array}$ & 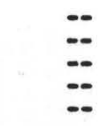 & $\begin{array}{l}= \\
=- \\
=\end{array}$ & $\begin{array}{r}0 \\
1400 \\
1900 \\
4600 \\
3500\end{array}$ & 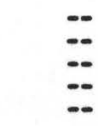 & $\begin{array}{l}= \\
= \\
=\end{array}$ & $\begin{array}{l}= \\
= \\
=\end{array}$ & 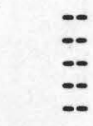 & 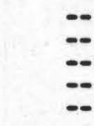 \\
\hline \multirow[t]{2}{*}{280246082343202} & $\begin{array}{l}71-12-27 \\
70-09-17 \\
70-10-22 \\
70-12-02 \\
71-01-14\end{array}$ & $\begin{array}{l}-- \\
20 \\
=-\end{array}$ & $\begin{array}{l}-= \\
0 \\
-\infty\end{array}$ & $\begin{array}{l}-- \\
-- \\
--\end{array}$ & $\begin{array}{r}30 \\
1100 \\
280 \\
450 \\
2300\end{array}$ & $\begin{array}{r}-- \\
0 \\
-\infty\end{array}$ & $\begin{array}{l}-- \\
10 \\
-\end{array}$ & $\begin{array}{l}0 \\
110 \\
--\end{array}$ & $\begin{array}{l}= \\
60 \\
-0\end{array}$ & $\begin{array}{l}-. \\
90 \\
--\end{array}$ \\
\hline & $\begin{array}{l}71-02-24 \\
71-03-31 \\
71-04-28 \\
71-06-15 \\
71-07-28\end{array}$ & $\begin{array}{l}= \\
=- \\
=\end{array}$ & 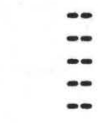 & $\begin{array}{l}= \\
=- \\
- \\
-\end{array}$ & $\begin{array}{l}130 \\
470 \\
2900 \\
1500 \\
1500\end{array}$ & $\begin{array}{l}= \\
= \\
= \\
=\end{array}$ & $\begin{array}{l}=- \\
=- \\
=-\end{array}$ & $\begin{array}{l}= \\
= \\
= \\
=\end{array}$ & $\begin{array}{l}\square \\
\because \\
\square\end{array}$ & 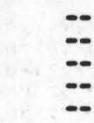 \\
\hline 280243082342602 & $\begin{array}{l}71-12-27 \\
70-09-17 \\
71-10-28\end{array}$ & $=$ & $=$ & $=$ & $\begin{array}{l}2700 \\
2000 \\
110\end{array}$ & $=$ & $=$ & $=$ & $=$ & $=$ \\
\hline 28024308234 & $70-10-22$ & 10 & - & 0 & $\because$ & 0 & - & 130 & 420 & 50 \\
\hline
\end{tabular}




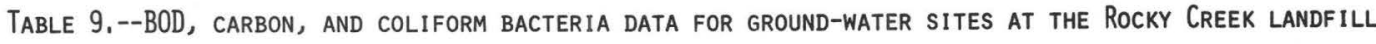

\begin{tabular}{|c|c|c|c|c|c|c|c|}
\hline STATION NUMBER & $\begin{array}{l}\text { DATE } \\
\text { OF } \\
\text { SAMPLE }\end{array}$ & $\begin{array}{l}\text { OXYGEN } \\
\text { DEMAND, } \\
\text { BIO- } \\
\text { CHEM- } \\
\text { ICAL, } \\
5 \text { DAY } \\
\text { (MG/L) }\end{array}$ & $\begin{array}{l}\text { CARBON, } \\
\text { ORGANIC } \\
\text { TOTAL } \\
\text { (MG/L } \\
\text { AS C) }\end{array}$ & $\begin{array}{l}\text { CARBON, } \\
\text { INOR- } \\
\text { GANIC, } \\
\text { TOTAL } \\
\text { (MG/L } \\
\text { AS C) }\end{array}$ & $\begin{array}{l}\text { COLI- } \\
\text { FORM, } \\
\text { TOTAL, } \\
\text { IMMEO. } \\
\text { (COLS. } \\
\text { PER } \\
100 \mathrm{ML} \text { ) }\end{array}$ & $\begin{array}{l}\text { COLI- } \\
\text { FORM, } \\
\text { FECAL, } \\
0.45 \\
\text { UM-MF } \\
\text { (COLS.' } \\
100 \mathrm{ML} \text { ) }\end{array}$ & $\begin{array}{l}\text { STREP- } \\
\text { TOCOCCI } \\
\text { FECAL, } \\
\text { (COLS. } \\
\text { PER } \\
100 \mathrm{ML} \text { ) }\end{array}$ \\
\hline $\begin{array}{l}280221082342901 \\
280226082342502 \\
280226082342501 \\
280226082341202 \\
280226082341201\end{array}$ & $\begin{array}{l}73-04-23 \\
73-04-26 \\
73-04-26 \\
73-04-24 \\
73-04-25\end{array}$ & $\begin{array}{l}- \\
\because- \\
= \\
=\end{array}$ & $\begin{array}{l}6.0 \\
6.0 \\
20 \\
9.0 \\
17\end{array}$ & $\begin{array}{l}40 \\
20 \\
34 \\
32 \\
44\end{array}$ & $\begin{array}{l}= \\
\because- \\
=-\end{array}$ & $\begin{array}{l}= \\
=- \\
= \\
=\end{array}$ & $\begin{array}{l}=- \\
\bar{z} \\
=-\end{array}$ \\
\hline 280227082343001 & $\begin{array}{l}73-06-07 \\
72-06-20 \\
73-01-23 \\
73-03-19 \\
72-06-06\end{array}$ & $\begin{array}{l}- \\
\because 9 \\
-9 \\
-\end{array}$ & $12^{--}$ & $\begin{array}{l}= \\
=- \\
=- \\
3.0\end{array}$ & $\begin{array}{r}130 \\
0 \\
2 \\
2 \\
070\end{array}$ & $\begin{array}{l}0 \\
0 \\
2 \\
2 \\
0\end{array}$ & $\begin{array}{r}-- \\
-- \\
0\end{array}$ \\
\hline 280231082343602 & $\begin{array}{l}73-04-24 \\
70-06-15 \\
71-04-28 \\
72-06-06 \\
73-04-24\end{array}$ & $\begin{array}{l}\because- \\
\because= \\
\because-\end{array}$ & $\begin{array}{r}2.0 \\
5.0 \\
18 \\
5.0 \\
7.5\end{array}$ & $\begin{array}{l}4.0 \\
20 \\
6.0 \\
15 \\
16\end{array}$ & $\begin{array}{r}-- \\
-0 \\
530 \\
--\end{array}$ & $\begin{array}{c}-- \\
-- \\
--\end{array}$ & $\begin{array}{c}-- \\
-- \\
--\end{array}$ \\
\hline 280231082343601 & $\begin{array}{l}70-06-15 \\
71-04-27 \\
72-06-06 \\
72-11-16 \\
73-01-23\end{array}$ & $\begin{array}{l}=- \\
=- \\
- \\
.7\end{array}$ & $\begin{array}{r}1.0 \\
9.0 \\
1.0 \\
--\end{array}$ & $\begin{array}{l}37 \\
29 \\
50 \\
-- \\
--\end{array}$ & $\begin{array}{r}-- \\
1000 \\
520 \\
7\end{array}$ & $\begin{array}{c}-- \\
- \\
0 \\
0 \\
0\end{array}$ & $\begin{array}{r}-- \\
-2 \\
0 \\
--\end{array}$ \\
\hline $\begin{array}{l}280233082340702 \\
280235082341701 \\
280236082340901 \\
280238082340902 \\
280238082340901\end{array}$ & $\begin{array}{l}73-10-03 \\
73-03-19 \\
73-08-10 \\
73-08-10 \\
73-08-10\end{array}$ & $\begin{array}{l}=- \\
=- \\
= \\
=\end{array}$ & $\begin{array}{l}=- \\
=- \\
=- \\
=\end{array}$ & $\begin{array}{l}=- \\
=- \\
=-\end{array}$ & $\begin{array}{r}2 \\
2 \\
9200 \\
170 \\
23\end{array}$ & $\begin{array}{r}2 \\
2 \\
3500 \\
22 \\
2\end{array}$ & $\begin{array}{l}-- \\
-- \\
--\end{array}$ \\
\hline $\begin{array}{l}280238082343201 \\
280238082342602\end{array}$ & $\begin{array}{l}73-10-02 \\
73-10-24 \\
73-12-18 \\
73-02-07 \\
72-08-08\end{array}$ & $\begin{array}{l}= \\
= \\
= \\
=\end{array}$ & $\begin{array}{l}= \\
=- \\
=\end{array}$ & $\begin{array}{l}=- \\
=- \\
=-\end{array}$ & $\begin{array}{r}33 \\
2 \\
0 \\
0 \\
40\end{array}$ & $\begin{array}{l}2 \\
2 \\
0 \\
0 \\
0\end{array}$ & 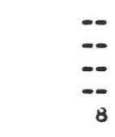 \\
\hline $\begin{array}{l}280238082335701 \\
280238082342204 \\
280240082340601\end{array}$ & $\begin{array}{l}72-09-06 \\
73-10-24 \\
73-04-26 \\
73-04-25 \\
73-06-07\end{array}$ & $\begin{array}{l}= \\
=- \\
=- \\
=\end{array}$ & $\begin{array}{l}-- \\
4.5 \\
11 \\
--\end{array}$ & $111^{--}$ & $\begin{array}{r}0 \\
2 \\
-- \\
-4\end{array}$ & $\begin{array}{r}0 \\
2 \\
-- \\
-0\end{array}$ & $\begin{array}{l}0 \\
=- \\
=- \\
=-\end{array}$ \\
\hline $\begin{array}{l}280240082341202 \\
280240082341201\end{array}$ & $\begin{array}{l}73-10-15 \\
73-08-10 \\
73-12-18 \\
73-08-10 \\
73-10-02\end{array}$ & $\begin{array}{l}= \\
=- \\
= \\
=\end{array}$ & $\begin{array}{l}= \\
\ddot{-} \\
=\end{array}$ & $\begin{array}{l}=- \\
\overline{-} \\
=-\end{array}$ & $\begin{array}{r}2 \\
11 \\
61 \\
7 \\
2\end{array}$ & $\begin{array}{r}2 \\
2 \\
<1 \\
2 \\
2\end{array}$ & $\begin{array}{l}=- \\
=- \\
=-\end{array}$ \\
\hline $\begin{array}{l}280239082343503 \\
280239082343502\end{array}$ & $\begin{array}{l}73-10-24 \\
72-06-22 \\
72-06-20 \\
72-08-08 \\
72-09-06\end{array}$ & $\begin{array}{l}=- \\
=- \\
= \\
=\end{array}$ & $\begin{array}{l}= \\
=- \\
= \\
=\end{array}$ & $\begin{array}{l}= \\
=- \\
= \\
=\end{array}$ & $\begin{array}{r}2 \\
330 \\
0 \\
-0 \\
8000\end{array}$ & $\begin{array}{r}2 \\
0 \\
0 \\
25 \\
0000\end{array}$ & $\begin{array}{r}-- \\
390 \\
21 \\
15 \\
900\end{array}$ \\
\hline & $\begin{array}{l}72-11-16 \\
73-01-23 \\
73-02-07 \\
73-02-26 \\
73-03-19\end{array}$ & $\begin{array}{l}-- \\
1.8 \\
-- \\
--\end{array}$ & $\begin{array}{l}= \\
= \\
= \\
=\end{array}$ & $\begin{array}{l}=- \\
=- \\
--\end{array}$ & $\begin{array}{r}870 \\
130 \\
-- \\
20 \\
20\end{array}$ & $\begin{array}{r}0 \\
2 \\
0 \\
20 \\
20\end{array}$ & $\begin{array}{r}1000 \\
=- \\
-\square \\
=\end{array}$ \\
\hline 280239082343501 & $\begin{array}{l}73-06-07 \\
72-06-20 \\
73-01-23 \\
73-02-26 \\
73-03-19\end{array}$ & $\begin{array}{l}-- \\
-\overline{1.2} \\
--\end{array}$ & $\begin{array}{l}= \\
\overline{-} \\
=\end{array}$ & $\begin{array}{l}=- \\
=- \\
=-\end{array}$ & $\begin{array}{r}170 \\
1 \\
79 \\
2 \\
2\end{array}$ & $\begin{array}{r}170 \\
0 \\
-- \\
2 \\
--\end{array}$ & $\begin{array}{r}-- \\
0 \\
-- \\
--\end{array}$ \\
\hline 280240082342601 & $\begin{array}{l}73-06-07 \\
72-09-06 \\
73-02-07 \\
73-02-26 \\
73-03-19\end{array}$ & $\begin{array}{l}= \\
=- \\
= \\
=\end{array}$ & $\begin{array}{l}= \\
=- \\
=\end{array}$ & $\begin{array}{l}= \\
=- \\
=-\end{array}$ & $\begin{array}{r}4 \\
0 \\
36 \\
2 \\
2\end{array}$ & $\begin{array}{l}0 \\
0 \\
0 \\
2 \\
2\end{array}$ & $\begin{array}{l}-- \\
0 \\
--\end{array}$ \\
\hline 280242082343502 & $\begin{array}{l}72-06-06 \\
72-06-20 \\
72-08-08 \\
72-09-06 \\
72-11-16\end{array}$ & $\begin{array}{l}= \\
=- \\
=- \\
=\end{array}$ & $\begin{array}{r}22 \\
=- \\
=- \\
=\end{array}$ & $\begin{array}{l}10 \\
=- \\
= \\
=-\end{array}$ & $\begin{array}{l}1100 \\
00 \\
2800 \\
5400 \\
7700\end{array}$ & $\begin{array}{r}0 \\
0 \\
1700 \\
16 \\
0\end{array}$ & $\begin{array}{r}3 \\
0 \\
2500 \\
550 \\
130\end{array}$ \\
\hline & $\begin{array}{l}73-01-23 \\
73-02-26 \\
73-06-07 \\
73-10-02 \\
73-10-24\end{array}$ & $\begin{array}{l}= \\
=- \\
=\end{array}$ & $\begin{array}{l}=- \\
=- \\
=-\end{array}$ & $\begin{array}{l}=- \\
=- \\
=-\end{array}$ & $\begin{array}{r}110 \\
2 \\
2400 \\
5 \\
2\end{array}$ & $\begin{array}{r}0 \\
20 \\
2400 \\
2 \\
2\end{array}$ & $\begin{array}{l}=- \\
=- \\
=-\end{array}$ \\
\hline 280242082343501 & $\begin{array}{l}73-12-18 \\
72-06-06 \\
72-06-20 \\
72-08-08 \\
73-01-23\end{array}$ & $\begin{array}{l}= \\
= \\
= \\
=\end{array}$ & $\begin{array}{l}-0 \\
1.0 \\
\overline{-} \\
--\end{array}$ & ${ }^{51}=$ & $\begin{array}{r}0 \\
390 \\
0 \\
130 \\
49\end{array}$ & $\begin{array}{l}<1 \\
- \\
0 \\
0 \\
2\end{array}$ & $\begin{array}{c}-- \\
0 \\
0\end{array}$ \\
\hline 280242082342601 & $\begin{array}{l}73-02-26 \\
73-10-02 \\
73-10-24 \\
73-12-18 \\
73-02-07\end{array}$ & $\begin{array}{l}= \\
=- \\
= \\
=\end{array}$ & $\begin{array}{l}= \\
= \\
=\end{array}$ & 13 & $\begin{array}{l}2 \\
2 \\
2 \\
1 \\
--\end{array}$ & $\begin{array}{l}2 \\
2 \\
2 \\
0 \\
0\end{array}$ & $\begin{array}{l}= \\
=- \\
=-\end{array}$ \\
\hline
\end{tabular}




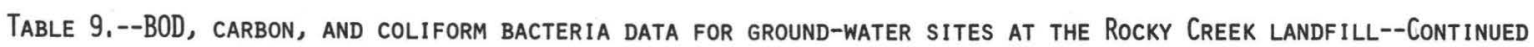

\begin{tabular}{|c|c|c|c|c|c|c|c|}
\hline STATION NUMBER & $\begin{array}{c}\text { DATE } \\
\text { OF } \\
\text { SAMPLE }\end{array}$ & $\begin{array}{l}\text { OXYGEN } \\
\text { DEMAND, } \\
\text { BIO- } \\
\text { CHEM- } \\
\text { ICAL, } \\
5 \text { DAY } \\
\text { (MG/L) }\end{array}$ & $\begin{array}{l}\text { CARBON, } \\
\text { ORGANIC } \\
\text { TOTAL } \\
\text { (MG/L } \\
\text { AS C) }\end{array}$ & $\begin{array}{l}\text { CARBON, } \\
\text { INOR- } \\
\text { GANIC, } \\
\text { TOTAL } \\
\text { (MG/L } \\
\text { AS C) }\end{array}$ & $\begin{array}{l}\text { COLI- } \\
\text { FORM, } \\
\text { TOTAL, } \\
\text { IMMED. } \\
\text { (COLS. } \\
\text { PER } \\
100 \mathrm{ML} \text { ) }\end{array}$ & $\begin{array}{l}\text { COLI- } \\
\text { FORM, } \\
\text { FECAL, } \\
0.45 \\
\text { UM-MF } \\
\text { (COLS.' } \\
100 \mathrm{ML} \text { ) }\end{array}$ & $\begin{array}{c}\text { STREP- } \\
\text { TOCOCCI } \\
\text { FECAL, } \\
\text { (COLS. } \\
\text { PER } \\
100 \mathrm{ML} \text { ) }\end{array}$ \\
\hline 280242082342601 & $73-08$ & - & -- & 1700 & -- & 1100 & - \\
\hline \multirow{4}{*}{280242082342602} & $\begin{array}{l}73-1 \\
72-0\end{array}$ & 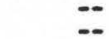 & 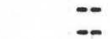 & 240 & 200 & $\begin{array}{l}2 \\
0\end{array}$ & 2200 \\
\hline & $72-11-06$ & - & - & - & - & 0 & - \\
\hline & $73-02-07$ & - & -- & -- & 13 & 0 & $\cdots$ \\
\hline & $73-10-02$ & -- & -- & -- & 24000 & 2 & -- \\
\hline
\end{tabular}


UNITED STATES DEPARTMENT OF THE INTERIOR GEOLOGICAL SURVEY

325 John Knox Rd--Suite F240

Tallahassee, Florida 32303
POSTAGE AND FEES PAID

U.S. DEPARTMENT OF THE INTERIOR

INT. 413 